Processamento de consultas SOLAP drill-across e com junção espacial em data warehouses geográficos

Jaqueline Joice Brito 



\title{
Processamento de consultas SOLAP drill-across e com junção espacial em data warehouses geográficos
}

\author{
Jaqueline Joice Brito
}

Orientadora: Profa. Dra. Cristina Dutra de Aguiar Ciferri

\begin{abstract}
Dissertação apresentada ao Instituto de Ciências Matemáticas e de Computação - ICMC-USP, como parte dos requisitos para obtenção do título de Mestre em Ciências - Ciências de Computação e Matemática Computacional. VERSÃO REVISADA
\end{abstract}


Ficha catalográfica elaborada pela Biblioteca Prof. Achille Bassi e Seção Técnica de Informática, ICMC/USP, com os dados fornecidos pelo(a) autor(a)

\begin{tabular}{|c|c|}
\hline \multirow[t]{3}{*}{ B862p } & $\begin{array}{l}\text { Brito, Jaqueline Joice } \\
\quad \text { Processamento de consultas SoLAP drill-across e } \\
\text { com junção espacial em data warehouses geográficos / } \\
\text { Jaqueline Joice Brito; orientadora Cristina Dutra de } \\
\text { Aguiar Ciferri. -- São Carlos, } 2012 \text {. } \\
\quad 110 \text { p. }\end{array}$ \\
\hline & $\begin{array}{l}\text { Dissertação (Mestrado - Programa de Pós-Graduação en } \\
\text { Ciências de Computação e Matemática Computacional) -- } \\
\text { Instituto de Ciências Matemáticas e de Computação, } \\
\text { Universidade de São Paulo, } 2012 \text {. }\end{array}$ \\
\hline & $\begin{array}{l}\text { 1. Data warehouses geográficos. 2. Processamento } \\
\text { de consultas SOLAP. 3. Drill-across. 4. Junção } \\
\text { espacial. I. Ciferri, Cristina Dutra de Aguiar, } \\
\text { orient. II. Título. }\end{array}$ \\
\hline
\end{tabular}




\section{Agradecimentos}

À minha mãe Eloísa e ao meu irmão lgor, pelo apoio, amor, carinho, incentivo e compreender minha ausência. Ao meu pai Armando (in memoriam), pelo amor, carinho e me dar à vida. Ao meu grande amigo e companheiro, Thiago, pelo amor, apoio e por tornar esta caminhada mais suave e divertida. À minha família, em especial aos meus avós, Ilidia e David, por sempre me apoiarem e acreditarem em mim.

À minha orientadora, Profa. Cristina D. A. Ciferri, pela oportunidade, ensinamentos, conselhos e dedicação com que me orientou neste trabalho. Acima de tudo, por sua amizade.

Aos estimados docentes Prof. Ricardo R. Ciferri, Prof. Thiago L. L. Siqueira e Profa. Valéria C. Times, pelas colaborações, orientações, sugestões e amizade.

Ao meu amigo Brenno Barbosa, pelas discussões, apoio e constante interesse em meu trabalho.

Aos colegas e docentes do GBDI, por compartilharem seus conhecimentos e proporcionarem um ambiente de trabalho agradável.

Ao ICMC-USP, pelo apoio institucional.

À CAPES, FAPESP e CNPQ, pelo apoio financeiro.

A todos que de alguma maneira contribuíram para a realização deste trabalho. 
Um data warehouse geográfico (DWG) é um banco de dados multidimensional, orientado a assunto, integrado, histórico, não-volátil e geralmente organizado em níveis de agregação. Além disso, também armazena dados espaciais em uma ou mais dimensões ou em pelo menos uma medida numérica. Visando oferecer suporte à tomada de decisão, é possível realizar em DWGs consultas SOLAP (spatial online analytical processing), isto é, consultas analíticas multidimensionais (e.g., drill-down, roll-up, drill-across) com predicados espaciais (e.g., intersecta, contém, está contido) definidos para range queries e junções espaciais. Um desafio no processamento dessas consultas é recuperar, de forma eficiente, dados espaciais e convencionais em DWGs muito volumosos. Na literatura, existem poucos índices voltados à indexação de DWGs, e ainda assim nenhum desses índices dedica-se a indexar consultas SOLAP drill-across e com junção espacial. Esta dissertação visa suprir essa limitação, por meio da proposta de estratégias para o processamento dessas consultas complexas. Para o processamento de consultas SOLAP drill-across foram propostas duas estratégias, Divide e Única, além da especificação de um conjunto de diretrizes que deve ser seguido para o projeto de um esquema de DWG que possibilite a execução dessas consultas e da especificação de classes de consultas. Para o processamento de consultas SOLAP com junção espacial foi proposta a estratégia SJB, além da identificação de quais características o esquema de DWG deve possuir para possibilitar a execução dessas consultas e da especificação do formato dessas consultas. A validação das estratégias propostas foi realizada por meio de testes de desempenho considerando diferentes configurações, sendo que os resultados obtidos foram contrastados com a execução de consultas do tipo junção estrela e o uso de visões materializadas. Os resultados mostraram que as estratégias propostas são muito eficientes. No processamento de consultas SOLAP drill-across, as estratégias Divide e Única mostraram uma redução no tempo de $82,7 \%$ a $98,6 \%$ com relação à junção estrela e ao uso de visões materializadas. No processamento de consultas SOLAP com junção espacial, a estratégia SJB garantiu uma melhora de desempenho na grande maioria das consultas executadas. Para essas consultas, o ganho de desempenho variou de $0,3 \%$ até $99,2 \%$.

Palavras-chave: Data warehouses geográficos. Processamento de consultas SOLAP. Drill-across. Junção espacial.

BRITO, J. J. Processamento de consultas SOLAP drill-across e com junção espacial em data warehouses geográficos. 2012. 110 p. Dissertação de Mestrado - Instituto de Ciências Matemáticas e de Computação, Universidade de São Paulo, São Carlos, 2012. 
A geographic data warehouse (GDW) is a special kind of multidimensional database. It is subject-oriented, integrated, historical, non-volatile and usually organized in levels of aggregation. Furthermore, a GDW also stores spatial data in one or more dimensions or at least in one numerical measure. Aiming at decision support, GDWs allow SOLAP (spatial online analytical processing) queries, i.e., multidimensional analytical queries (e.g., drill-down, roll-up, drill-across) extended with spatial predicates (e.g., intersects, contains, is contained) defined for range and spatial join queries. A challenging issue related to the processing of these complex queries is how to recover spatial and conventional data stored in huge GDWs efficiently. In the literature, there are few access methods dedicated to index GDWs, and none of these methods focus on drill-across and spatial join SOLAP queries. In this master's thesis, we propose novel strategies for processing these complex queries. We introduce two strategies for processing SOLAP drill-across queries (namely, Divide and Unique), define a set of guidelines for the design of a GDW schema that enables the execution of these queries, and determine a set of classes of these queries to be issued over a GDW schema that follows the proposed guidelines. As for the processing of spatial join SOLAP queries, we propose the SJB strategy, and also identify the characteristics of a DWG schema that enables the execution of these queries as well as define the format of these queries. We validated the proposed strategies through performance tests that compared them with the star join computation and the use of materialized views. The obtained results showed that our strategies are very efficient. Regarding the SOLAP drill-across queries, the Divide and Unique strategies showed a time reduction that ranged from $82,7 \%$ to $98,6 \%$ with respect to star join computation and the use of materialized views. Regarding the SOLAP spatial join queries, the SJB strategy guaranteed best results for most of the analyzed queries. For these queries, the performance gain of the SJB strategy ranged from $0,3 \%$ to $99,2 \%$ over the star join computation and the use of materialized view.

Keywords: Geographic data warehouses. SOLAP query processing. Drill-across. Spatial join.

BRITO, J. J. Processing of drill-across and spatial-join SOLAP queries over geographic data warehouses. 2012. 110 p. Dissertação de Mestrado - Instituto de Ciências Matemáticas e de Computação, Universidade de São Paulo, São Carlos, 2012. 
2.1 Cubo de dados multidimensional do exemplo da cadeia de lojas. . . . . . . . . 7

2.2 Grafo de derivação das dimensões do exemplo da cadeia de lojas. . . . . . . . . . 8

2.3 Esquema estrela do SSB . . . . . . . . . . . . . . . . . . . . . . . . . . . . . . . 9

2.4 Normalização da dimensão customer do SSB. . . . . . . . . . . . . . . . . . . . . 10

2.5 Constelação de fatos extraída do benchmark TPC-H. . . . . . . . . . . . . . . 10

2.6 Exemplo de operações drill-down e roll-up. . . . . . . . . . . . . . . . . . . . 11

2.7 Exemplo de operação pivot. . . . . . . . . . . . . . . . . . . . . . . . . 12

2.8 Exemplo de operação slice and dice. . . . . . . . . . . . . . . . . . . . . . . . 12

2.9 New Geographic Hybrid SSB (NewGHSSB). . . . . . . . . . . . . . . . . . . . . 14

2.10 Exemplos de tipos de dados espaciais. . . . . . . . . . . . . . . . . . . . . . . 14

2.11 Subtipos de range queries - adaptada de (Ciferri, 2002b) . . . . . . . . . 16

3.1 Minimum Bounding Rectangle (MBR) de um objeto espacial. . . . . . . . . . 20

3.2 Exemplo de R-Tree. . . . . . . . . . . . . . . . . . . . . . . . 20

3.3 Índice de Projeção - adaptada de (Siqueira et al., 2009a). . . . . . . . . . . 24

3.4 Índice Bitmap de junção - adaptada de (Siqueira et al., 2009a). . . . . . . . . 26

4.1 aR-tree - adaptada de (Papadias et al., 2001) . . . . . . . . . . . . 30

4.2 Grafo de derivação da aR-tree - extraída de (Papadias et al., 2001). . . . . . . 31

4.3 a3DR-tree . . . . . . . . . . . . . . . . . . . . . 33

4.4 aRB-tree - adaptada de (Papadias et al., 2002). . . . . . . . . . . . . . 34

4.5 Exemplo de janela de consulta - extraída de (Papadias et al., 2002). . . . . . 34

4.6 Índices SJALI e SJELI - adaptada de (Mohan et al., 2008). . . . . . . . . . . 35

4.7 Estrutura do SB-index - extraída de (Siqueira, 2009) . . . . . . . . . . . . . 37

4.8 Construção do SB-index - extraída de (Siqueira, 2009) . . . . . . . . . . . . . 38

4.9 Processamento de consultas pelo SB-index - extraída de (Siqueira, 2009). . . . 38

4.10 Estrutura do HSB-index - extraída de (Siqueira et al., 2012) . . . . . . . . . . . 39

5.1 Processamento de uma consulta SOLAP drill-across que incide sobre uma constelação de fatos com dois esquemas estrelas, $A$ e $B$, sendo realizado pela estratégia Divide.

5.2 Processamento de uma consulta SOLAP drill-across que incide sobre uma constelação de fatos sendo realizado pela estratégia Única.

5.3 Esquema SpatialDrillAcross construído a partir de uma adaptação dos esquemas do benchmark TPC-H e do NewGHSSB . . . . . . . . . . . . . . . . . . 53

5.4 Esquema SpatialDrillAcross modificado. . . . . . . . . . . . . . . . . 55

5.5 Dados da tabela de dimensão espacial Region. . . . . . . . . . . . . . . . . . . 56

5.6 Template das classes de consultas SOLAP drill-across. . . . . . . . . . . . . . . 58

5.7 Valores de granularidade e predicado espacial paras as classes Q1, Q2 e Q3. . 58

5.8 Comparação do desempenho entre junção estrela, visões materializadas $\mathrm{V}_{D}$ e $\mathrm{V}_{U}$ e as estratégias Divide e Única. 
5.9 Desempenho das estratégias Divide e Única variando a seletividade das consultas. 65

5.10 Desempenho das estratégias Divide e Única variando o fator de escala (SF) convencional. . . . . . . . . . . . . . . . . . . 66

5.11 Desempenho das estratégias Divide e Única variando o fator de escala espacial (SSF) . . . . . . . . . . . . . . . . . . . . . . . . . . . . . .

5.12 Razão $T_{U} / T_{D}$ : variação da proporção entre as tabelas de fatos Partsupp e Lineitem.

5.13 Razão $\mathrm{T}_{U} / \mathrm{T}_{D}$ : variação da proporção entre as tabelas de fatos Partsupp e Lineitem e da seletividade das consultas.

5.14 Razão $\mathrm{T}_{U} / \mathrm{T}_{D}$ : variação da proporção entre as tabelas de fatos Partsupp e Lineitem e do fator de escala (SF) convencional. . . . . . . . . . . . . . . . 74

5.15 Razão $\mathrm{T}_{U} / \mathrm{T}_{D}$ : variação da proporção entre as tabelas de fatos Partsupp e Lineitem e do fator de escala espacial (SSF) . . . . . . . . . . . . . . . . . 75

6.1 Esquema SpatialDrillAcrosst. . . . . . . . . . . . . . . . . . . . . . . 83

6.2 Dados espaciais das tabelas City, Nation, Region, River e Port com $S S F=1 . \quad 84$

6.3 Template dos conjuntos de consultas SOLAP com junção espacial. . . . . . . . 84

6.4 Valores de granularidade e predicado espacial dos conjuntos de consultas SOLAP com junção espacial Q1, Q2, Q3, Q4, Q5 e Q6. . . . . . . . . . . . . 85

6.5 Comparação do desempenho entre junção estrela, visão materializada e a estratégia SJB para os conjuntos Q1, Q2 e Q3.

6.6 Comparação do desempenho entre junção estrela, visão materializada e a estratégia SJB para os conjuntos Q4, Q5 e Q6. . . . . . . . . . . . . . . . . 92

6.7 Comparação do desempenho entre visão materializada e a estratégia SJB para os conjuntos Q1, Q2 e Q3 variando o fator de escala (SF) convencional.

6.8 Comparação do desempenho entre visão materializada e a estratégia SJB para os conjuntos Q4, Q5 e Q6 variando o fator de escala (SF) convencional.

6.9 Comparação do desempenho entre visão materializada e a estratégia SJB para os conjuntos Q1, Q2 e Q3 variando o fator de escala espacial (SSF).

6.10 Comparação do desempenho entre visão materializada e a estratégia SJB para os conjuntos Q4, Q5 e Q6 variando o fator de escala espacial (SSF). 


\section{Lista de Tabelas}

5.1 Seletividade dos Conjuntos de Janelas de Consulta. . . . . . . . . . . . . . . 60

6.1 Configurações dos predicados convencionais. . . . . . . . . . . . . . . . . 86

6.2 Porcentagem de tuplas retornadas pelo predicado espacial de cada consulta. . . 87 


\section{Lista de Algoritmos}

$1 \quad$ DivideContruirÍndices $(C F, m, n) \ldots \ldots \ldots \ldots$

2 DivideProcessarConsulta $\left(Q, C F, I B J_{1}, \ldots, I B J_{m}, S B_{1}, \ldots, S B_{n}\right) \ldots \ldots . .50$

3 ÚnicaContruirÍndices $(C F, n) \ldots \ldots \ldots \ldots \ldots$

4 ÚnicaProcessarConsulta $\left(Q, C F, I B J, S B_{1}, \ldots, S B_{n}\right) \ldots \ldots \ldots$

$5 \quad$ SJBContruirÍndices $(E E, n) \ldots \ldots \ldots \ldots \ldots$

6 SJBProcessarConsulta $\left(Q, E E, I B J, S B_{1}, \ldots, S B_{n}\right) \ldots \ldots \ldots \ldots$ 


\section{Lista de Siglas e Acrônimos}

a3DR-tree aggreagate 3-Dimensional R-tree

aR-tree aggreagate $R$-tree

aRB-tree aggreagate RB-tree

DW Data Warehouse

DWG Data Warehouse Geográfico

HSB-index Hierarchical Spatial Bitmap index

JC Janela de Consulta

MBR Minimum Bounding Rectangle

MOLAP Multidimensional OLAP

NewGHSSB New Geographic Hybrid Star Schema Benchmark

OLAP On-Line Analytical Processing

OLTP On-Line Transaction Processing

ROLAP Relational OLAP

SB-index Spatial Bitmap index

SGBDRs Sistemas de Gerenciamento de Bancos de Dados Relacionais

SIGs Sistemas de Informações Geográficas

SJALI Self-Join Adjacency List Index

SJELI Self-Join Edge List Index

SOLAP Spatial OnLine Analytical Processing

SSB Star Schema Benchmark

SSD Sistemas de Suporte à Decisão

WAH Word-Aligned Hybrid Code 
Lista de Figuras $\quad$ vii

Lista de Tabelas $\quad$ ix

Lista de Algoritmos $\quad$ xi

Lista de Siglas e Acrônimos $\quad$ xiii

1 Introdução $\quad \mathbf{1}$

1.1 Motivações e Contribuições . . . . . . . . . . . . . . . . . . . . . . 2

1.2 Estrutura da Dissertação . . . . . . . . . . . . . . . . . . . . . . . . 4

2 Data Warehouse 5

2.1 Data Warehouse Convencional . . . . . . . . . . . . . . . . . . . . . . 5

2.1.1 Modelagem Multidimensional . . . . . . . . . . . . . . . . 6

2.1 .2 Níveis de Agregação . . . . . . . . . . . . . . . . . . . . . . . . . . . . 7

2.1 .3 Visão Materializada . . . . . . . . . . . . . . . . . . . . . . . . . 8

2.1.4 Representação Lógica dos Dados . . . . . . . . . . . . . . . . . . . . 8

2.1 .5 Operações OLAP . . . . . . . . . . . . . . . . . . . . . . . . . 11

2.2 Data Warehouse Geográfico . . . . . . . . . . . . . . . . . . . . . . . . . 13

2.2.1 Tipos de Dados Espaciais . . . . . . . . . . . . . . . . . . . . . . . . . . . . . . . . . . . . . 15

2.2 .2 Consultas SOLAP . . . . . . . . . . . . . . . . . . . 15

2.3 Considerações Finais . . . . . . . . . . . . . . . . . . . . . . . . . 17

3 Índices 19

3.1 Índices Espaciais . . . . . . . . . . . . . . . . . . . . . . . . . . . . . . . . . . . 19

3.1 .1 R-Tree . . . . . . . . . . . . . . . . . . . . . . . . . 19

3.1 .2 R*-Tree . . . . . . . . . . . . . . . . . . . . . 22

3.1 .3 Algoritmos de Junção Espacial . . . . . . . . . . . . . . . . . . . 22

3.2 Índices para Data Warehouses Convencionais . . . . . . . . . . . . . . . . . . 24

3.2 .1 Índice de Projeção . . . . . . . . . . . . . . . . . . . . . . . . . . . . 24

3.2 .2 Índice Bitmap de Junção . . . . . . . . . . . . . . . . . . . . . . 25

3.3 Considerações Finais . . . . . . . . . . . . . . . . . . . . . . . . . . . . . . . . . 27

4 Índices para Data Warehouses Geográficos 29

4.1 aR-tree . . . . . . . . . . . . . . . . . . . . . . . . . . . . . . . . . 29

4.1.1 Processamento de Consultas . . . . . . . . . . . . . . . . . . 31

4.2 a3DR-tree e aRB-tree . . . . . . . . . . . . . . . . . . . . . . . 31

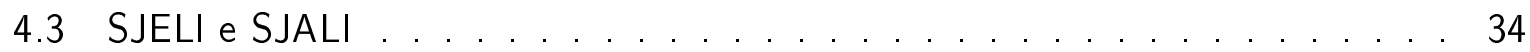

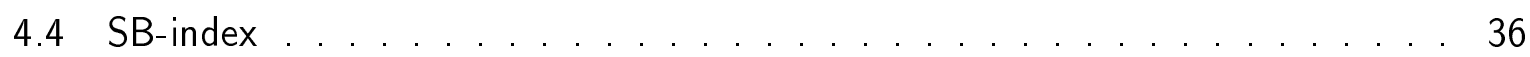


4.4.1 Estrutura de Dados e Construção do Índice . . . . . . . . . . . . . . . 36

4.4.2 Processamento de Consultas . . . . . . . . . . . . . . . . . . . . . 37

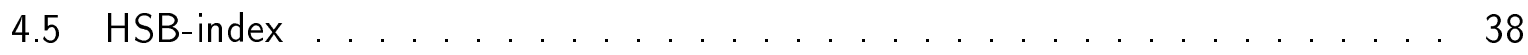

4.6 Considerações Finais . . . . . . . . . . . . . . . . . . . . . . . . . . . . . . . . . 40

5 Processamento de Consultas SOLAP Drill-across 43

5.1 Proposta . . . . . . . . . . . . . . . . . . . . . . 44

5.1.1 Diretrizes do Esquema . . . . . . . . . . . . . . . . . . . . . . . . . . 44

5.1 .2 Classes de Consultas . . . . . . . . . . . . . . . . . . . . . 45

5.1 .3 Estratégia Divide . . . . . . . . . . . . . . . . . . . . . . . . . 48

5.1 .4 Estratégia Única . . . . . . . . . . . . . . . . . . . . . . . . . . . . 50

5.2 Testes de Desempenho . . . . . . . . . . . . . . . . . . . . . . . . . 52

5.2 .1 Configuração dos Testes . . . . . . . . . . . . . . . . . . . . 52

5.2.2 Comparação entre Junção Estrela, Visão Materializada e as Estratégias

Divide e Única . . . . . . . . . . . . . . . . . . . . . . . . . . 60

5.2.3 Divide e Única: Variando a Seletividade das Consultas . . . . . . . . . 61

5.2.4 Divide e Única: Variando o Fator de Escala Convencional . . . . . . . 61

5.2.5 Divide e Única: Variando o Fator de Escala Espacial . . . . . . . . . . 62

5.2.6 Divide e Única: Variando a Proporção entre Tabelas de Fatos . . . . . 68

5.2.7 Divide e Única: Variando a Proporção entre Tabelas de Fatos e a

Seletividade das Consultas . . . . . . . . . . . . . . . . . . . 69

5.2.8 Divide e Única: Variando a Proporção entre Tabelas de Fatos e o Fator de Escala Convencional . . . . . . . . . . . . . . . . . . . . . 70

5.2.9 Divide e Única: Variando a Proporção entre Tabelas de Fatos e o Fator de Escala Espacial . . . . . . . . . . . . . . . . . . . . . . 71

5.3 Considerações Finais . . . . . . . . . . . . . . . . . . . . . . . . . . . . . . . 76

6 Processamento de Consultas SOLAP com Junção Espacial 77

6.1 Proposta . . . . . . . . . . . . . . . . . . . . . . . 77

6.1 .1 Características do Esquema . . . . . . . . . . . . . . . . . . . . 78

6.1 .2 Formato de Consultas . . . . . . . . . . . . . . . . . . . . . . 78

6.1 .3 Estratégia SJB . . . . . . . . . . . . . . . . . . . . . . . . . . . . . . 80

6.2 Testes de Desempenho . . . . . . . . . . . . . . . . . . . . . . . . . . . . . . 81

6.2 .1 Configuração dos Testes . . . . . . . . . . . . . . . . . . . . . . . 82

6.2.2 Comparação entre Junção Estrela, Visão Materializada e a Estratégia SJB . . . . . . . . . . . . . . . . . 88

6.2.3 SJB: Variando o Fator de Escala Convencional . . . . . . . . . . . . . 89

6.2.4 SJB: Variando o Fator de Escala Espacial . . . . . . . . . . . . . . . 90

6.3 Considerações Finais . . . . . . . . . . . . . . . . . . . . . . . . . . . . . . . . 97

7 Conclusões 99

7.1 Contribuições . . . . . . . . . . . . . . . . . . . . . . . . . . . . . . 101

7.2 Trabalhos Futuros . . . . . . . . . . . . . . . . . . . . 101

$\begin{array}{ll}\text { Referências Bibliográficas } & 103\end{array}$ 


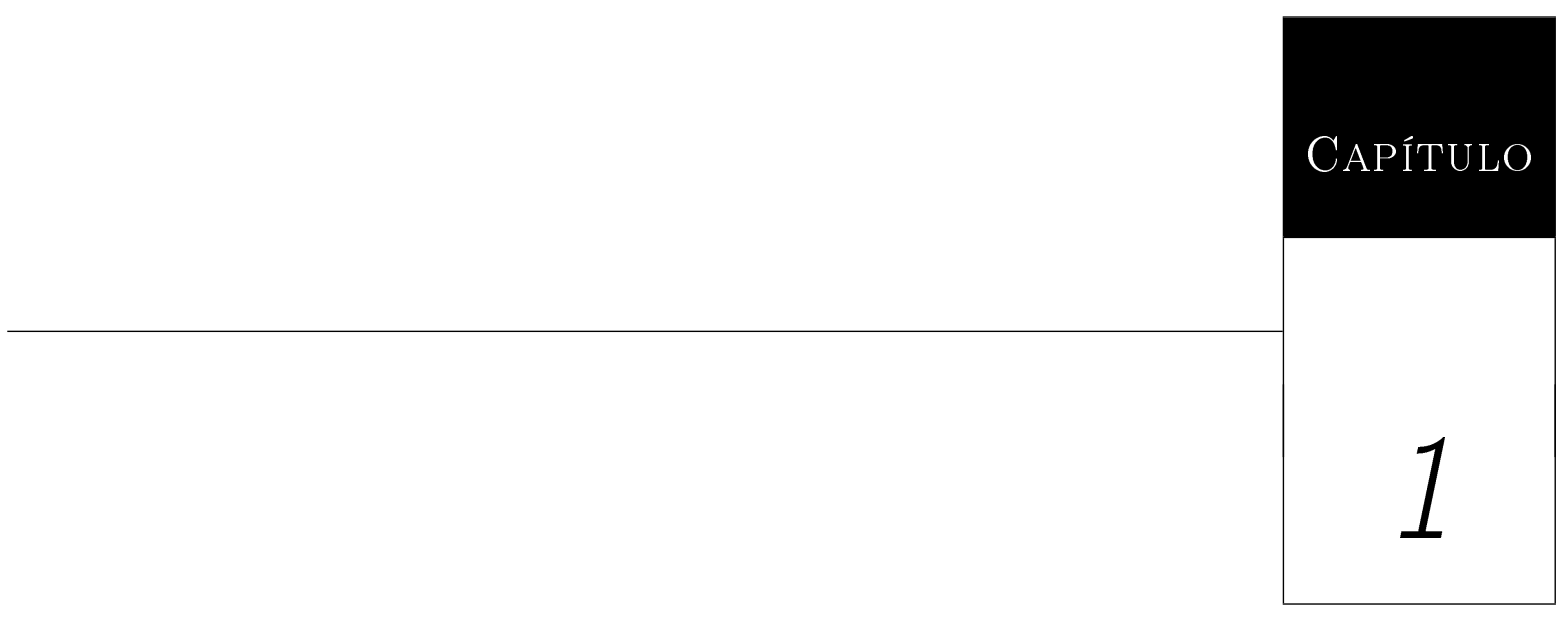

\section{Introdução}

Um data warehouse (DW) é um banco de dados especialmente organizado para armazenar dados orientados a assunto, integrados, históricos e não-voláteis. Além disso, ele é multidimensional, ou seja, um DW possui um conjunto de medidas numéricas, que são os objetos de análise relevantes ao negócio, e um conjunto de dimensões, as quais determinam o contexto para as medidas numéricas. Outra característica do DW é que ele é usualmente organizado em níveis de agregação (Chaudhuri e Dayal, 1997; Ciferri, 2000; Golfarelli e Rizzi, 2009; Golfarelli et al., 2004a; Inmon, 2002; Samtani et al., 1998; Vassiliadis, 2000; Widom, 1995; Wu e Buchmann, 1997).

Assim como um DW, um data warehouse geográfico (DWG) é um banco de dados orientado a assunto, integrado, histórico, não-volátil, multidimensional e geralmente organizado em níveis de agregação. Adicionalmente, um DWG armazena dados espaciais em uma ou mais dimensões ou em pelo menos uma medida numérica (Bimonte et al., 2005; Fidalgo et al., 2004; Malinowski e Zimányi, 2004, 2005; Sampaio et al., 2006; Silva et al., 2006, 2008; Stefanovic et al., 2000). Os dados espaciais são abstrações geométricas e topológicas dos fenômenos do mundo real (e.g., região, bairro, bacia petrolífera). Ponto, linha e polígono são exemplos de tipos de dados espaciais. Visando oferecer suporte à tomada de decisão, DWGs permitem consultas SOLAP (spatial online analytical processing), isto é, consultas analíticas multidimensionais (e.g., drill-down, roll-up, drill-across) com predicado espacial (e.g., intersecta, contém, está contido) definido para range queries e junção espacial. 
Um desafio do processamento de consultas SOLAP é recuperar eficientemente dados espaciais e convencionais armazenados em DWGs extremamente volumosos. Embora existam diversos métodos na literatura que visem melhorar o desempenho de consultas em DWs e DWGs, tais como visão materializada (Agrawal et al., 2000; Baikousi e Vassiliadis, 2009; Hung et al., 2007; Kotidis e Roussopoulos, 2001), fragmentação horizontal e vertical de dados (Bellatreche e Woameno, 2009; Ciferri et al., 2007; Golfarelli et al., 2004b), particionamento dos dados para processamento paralelo (Benkrid et al., 2008; Costa e Madeira, 2004) e índices (Johnson e Shasha, 1997; Papadias et al., 2001, 2002; Sarawagi, 1997; Siqueira et al., 2009b, 2008, 2009a), não existem na literatura trabalhos voltados ao processamento de consultas SOLAP drill-across e consultas SOLAP com junção espacial. Surge, então, a necessidade de se propor métodos de processamento que enfoquem essas consultas SOLAP complexas. Esse é o objetivo desta dissertação de mestrado.

\subsection{Motivações e Contribuições}

A presente dissertação visa oferecer suporte à tomada de decisão em DWGs de forma eficiente. Mais detalhadamente, esta dissertação tem como objetivo propor estratégias de processamento de consultas SOLAP drill-across e consultas SOLAP com junção espacial.

As motivações para o desenvolvimento desta dissertação podem ser observadas sob duas perspectivas. Na primeira perspectiva, consultas drill-across são consultas muito importantes em DWs porque permitem a realização de análises comparativas que cruzam dados provenientes de diferentes relações. Na modelagem multidimensional utilizada em DWs relacionais, relações que contêm esses dados são denominadas tabelas de fatos. Essas tabelas armazenam diversas medidas numéricas e referências para tabelas de dimensão, as quais são, por sua vez, relações que contêm dados descritivos das dimensões que contextualizam as medidas numéricas. Assim, consultas drill-across recuperam medidas numéricas de diferentes tabelas de fatos que compartilham uma ou mais dimensões, permitindo a manipulação e a comparação dessas medidas.

Como um exemplo de consulta drill-across com predicado espacial, considere um DWG que contém dados históricos relativos a pedidos e vendas de uma corporação. Suponha que os dados referentes à quantidade de produtos vendidos por filiais de uma empresa em uma certa data estejam localizados em uma tabela de fatos, enquanto os dados sobre os valores de custo e venda desses produtos em uma certa data e filial estejam localizados em outra tabela de fatos. Suponha também que os dados das filiais sejam dados espaciais. Consultas drill-across envolvendo o predicado espacial intersecta são necessárias para responder às requisições do tipo: "Calcule o lucro mensal por produto e filial no mês de setembro cuja localização da filial intersecta uma janela de consulta". As tabelas de fatos compartilham as dimensões produto, data e filial, tornando possível a comparação das medidas valor de custo e valor de venda com unidades vendidas. 
Em geral, DWs armazenam milhões de tuplas nas tabelas de fatos. Isso já prejudica a eficiência de análises comparativas via consultas drill-across tradicionalmente realizadas contra esses DWs. Com a adição de dados espaciais em DWGs e de predicados espaciais a essas consultas, o problema torna-se ainda mais custoso, motivando o desenvolvimento de estratégias que otimizem o processamento dessas consultas SOLAP complexas. Visando enfocar esse desafio, nesta dissertação são introduzidas as seguintes contribuições:

- A especificação de um conjunto de diretrizes que deve ser seguido para o projeto de um esquema de DWG que possibilite a execução de consultas SOLAP drill-across.

- A especificação de classes de consultas SOLAP drill-across que introduzem diferentes custos de processamento, de forma que essas consultas sejam executadas sobre um DWG projetado de acordo com as diretrizes propostas.

- A proposta de duas estratégias baseadas no uso de índice para o processamento de consultas SOLAP drill-across em DWGs, chamadas de Divide e Única.

A segunda perspectiva diz respeito à junção espacial. Consultas com junção espacial são importantes em DWGs porque proporcionam análises de dados georreferenciados de acordo com um relacionamento espacial entre esses dados. Considerando o DWG do exemplo corrente, um exemplo de consulta SOLAP com junção espacial é "Qual o lucro anual por produto por filial em vendas realizadas em filiais localizados a menos de $100 \mathrm{~km}$ da filial de São Carlos"?.

Em bancos de dados geográficos, a operação de junção espacial seleciona um subconjunto do produto cartesiano de dois conjuntos de objetos espaciais de acordo com um predicado espacial. Em DWGs, o processamento da junção espacial torna-se muito mais custoso, desde que ela é realizada sobre conjuntos volumosos de objetos espaciais. Isso deve-se ao fato de o produto cartesiano gerado por esses conjuntos resultar em um grande número de pares de objetos espaciais a serem analisados pelo predicado espacial. Desse modo, a necessidade de índices e algoritmos que realizem consultas SOLAP com junção espacial de forma eficiente torna-se ainda maior. Visando enfocar esse desafio, nesta dissertação são introduzidas as seguintes contribuições:

- A identificação de quais características o esquema de DWG deve possuir para possibilitar a execução de consultas SOLAP com junção espacial.

- A especificação do formato de consultas SOLAP com junção espacial, de forma que essas consultas sejam executadas sobre um DWG projetado de acordo com as características identificadas.

- A proposta de uma estratégia baseada no uso de índice para o processamento de consultas SOLAP com junção espacial, chamada de SJB. 
A validação das estratégias de processamento de consultas SOLAP drill-across e consultas SOLAP com junção espacial propostas nesta dissertação foi realizada por meio de testes de desempenho considerando diferentes volumes de dados armazenados no DWG e diferentes seletividades de consultas. Os resultados obtidos foram contrastados com a execução de consultas do tipo junção estrela e com o uso de visões materializadas. Os resultados mostraram que as estratégias propostas são muito eficientes. No processamento de consultas SOLAP drillacross, as estratégias Divide e Única mostraram uma redução no tempo de 82,7\% a 98,6\% com relação à junção estrela e ao uso de visões materializadas. No processamento de consultas SOLAP com junção espacial, a estratégia SJB garantiu uma melhora de desempenho na grande maioria das consultas executadas. Para essas consultas, o ganho de desempenho variou de $0,3 \%$ até $99,2 \%$ com relação à junção estrela e ao uso de visões materializadas. Resultados preliminares relacionados ao processamento de consultas SOLAP drill-across foram publicados no evento científico International Conference on Data Warehousing and Knowledge Discovery (DaWaK), o qual possui classificação CAPES QUALIS Ciência da Computação B1 (Brito et al., 2011).

\subsection{Estrutura da Dissertação}

Além deste capítulo de introdução, esta dissertação está estruturada em mais seis capítulos:

- Capítulo 2: são detalhados conceitos básicos de DWs convencionais e geográficos, os quais são necessários para o entendimento desta dissertação.

- Capítulo 3: são descritos tópicos relacionados a índices espaciais (e.g., R-Tree) e índices para DWs (e.g., índice Bitmap de junção).

- Capítulo 4: são resumidos trabalhos correlatos voltados à indexação de DWGs.

- Capítulo 5: é apresentada a proposta para o processamento de consultas SOLAP drillacross.

- Capítulo 6: é apresentada a proposta para o processamento de consultas SOLAP com junção espacial.

- Capítulo 7: são destacadas as conclusões da dissertação, contribuições e trabalhos futuros. 


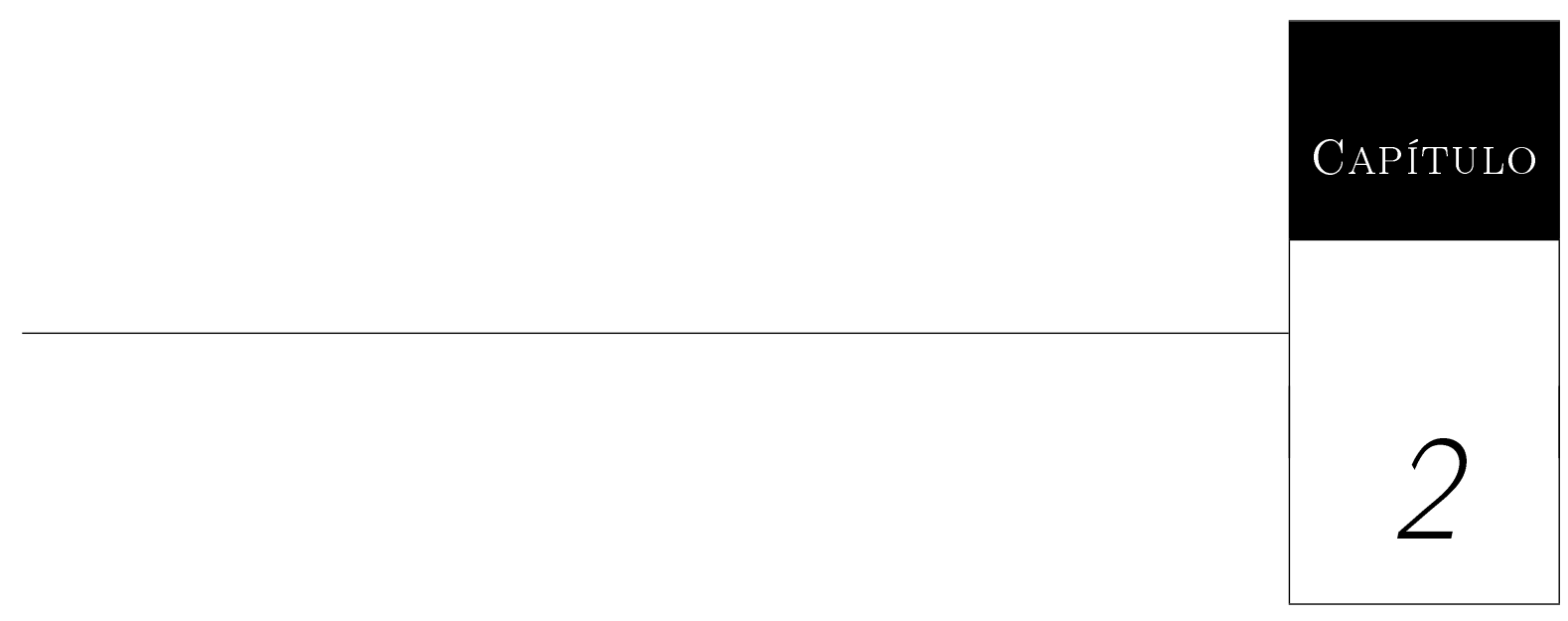

\section{Data Warehouse}

A grande quantidade de dados armazenados por organizações ao longo dos anos abriu uma nova perspectiva para o desenvolvimento de ferramentas que possibilitem a análise desse grande volume de dados. É nesse cenário que surgem os data warehouses e a tecnologia OLAP (online analytical processing) para auxiliar o processo de tomada de decisão. Neste capítulo são detalhados conceitos básicos relacionados a data warehouses convencionais e geográficos. Na seção 2.1 são descritos os principais conceitos envolvendo data warehouses convencionais (e.g., modelagem multidimensional, operações OLAP, visão materializada) e na seção 2.2 são descritos data warehouses geográficos, bem como tipos de dados e consultas espaciais. O capítulo é finalizado na seção 2.3 , com as considerações finais.

\subsection{Data Warehouse Convencional}

Um DW é um banco de dados especialmente organizado para armazenar dados orientados a assunto, integrados, históricos e não-voláteis. Os dados são orientados a assunto desde que referem-se a um negócio de interesse de uma organização, como vendas e transporte de produtos. Outra característica é que os dados são integrados. Diferentes provedores de informações fornecem, muitas vezes de forma incompatível, dados a um DW. Antes dos dados serem carregados no DW, um processo de integração é empregado para garantir a confiabilidade e a consistência dos dados armazenados. 
DWs também são organizados de maneira histórica, ou seja, os dados referenciam um certo período do tempo. No ambiente operacional, composto pelos sistemas OLTP (online transaction processing), apenas o estado atual dos dados é relevante. Modificações sobre esses dados geram novas entradas no ambiente informacional (i.e., sistemas OLAP), gerando um histórico sobre as operações realizadas no ambiente operacional. Esse histórico permite que análises detalhadas sejam realizadas, fornecendo informações estratégicas que auxiliem no processo de tomada de decisão. Essa característica indica o quão rápido pode ser o crescimento do volume de dados de um DW.

Uma vez que os dados são carregados no DW, raramente eles são modificados ou removidos. Por essa característica, os dados do DW são caracterizados como não-voláteis. Em um DW, os dados são geralmente removidos após um longo período de tempo. Isso ocorre quando não houver mais espaço de armazenamento ou quando essas informações não forem mais relevantes para as análises realizadas pelos usuários de sistemas de suporte à decisão (SSD).

Uma vez definido o que é um DW, o restante desta seção está estruturada da seguinte forma. Na seção 2.1.1 é descrita a modelagem multidimensional. Nas seções 2.1.2 e 2.1.3 são definidos os conceitos de níveis de agregação e de visão materializada, respectivamente. A representação lógica dos dados é detalhada na seção 2.1.4, enquanto que consultas OLAP são descritas na seção 2.1.5.

\subsubsection{Modelagem Multidimensional}

Dados relativos às vendas de uma cadeia de lojas podem ser descritos sob vários aspectos: tempo em que a venda foi realizada, produto vendido e filial em que a venda foi realizada. Esses diferentes aspectos são chamados de dimensões e por esse motivo os dados de um DW são considerados multidimensionais (Chaudhuri e Dayal, 1997). Esse modelo de organização facilita a análise e visualização dos dados, fornecendo diferentes perspectivas aos usuários de SSD.

Uma consulta que pode ser realizada é: "Qual o total de unidades vendidas por produto e filial ao longo do tempo?". Nesse exemplo, a perspectiva de tempo pode ser descrita por vários atributos, como hora, dia, mês, semestre, ano. Esses atributos estabelecem relações, originando o que é chamado de hierarquia de atributos. Utilizando como base a notação introduzida por (Harinarayan et al., 1996), a hierarquia gerada por esses atributos é definida da seguinte forma: ano $\preceq$ semestre $\preceq$ mês $\preceq$ dia $\preceq$ hora. Isso significa que horas podem ser agregadas em um dia, dias podem ser agregados em um mês, meses podem ser agregados em um semestre e semestres podem ser agregados em um ano. Essas hierarquias de atributos definem diferentes níveis de agregação e, consequentemente, diferentes níveis de granularidade. Por exemplo, a granularidade da cadeia de lojas pode ser vendas diárias, vendas mensais, vendas semestrais ou vendas anuais. 
Os dados de um DW são orientados a um processo de negócio no qual há interesse em realizar-se análises, ou seja, orientados a um objeto de análise. No exemplo da cadeia de lojas, o objeto de análise pode ser o número de unidades vendidas de produtos. O objeto de análise de um negócio é chamado de medida numérica. Medidas numéricas são funções das dimensões, ou seja, dada uma certa filial, um certo produto e um determinado tempo, tem-se um valor de medida numérica associado, isto é, um número de unidades vendidas. A modelagem realizada por meio de um conjunto de dimensões e medidas numéricas é denominada multidimensional.

A representação gráfica dos dados utilizada por essa modelagem é a de um cubo de dados multidimensional. Essa representação facilita a compreensão da organização e manipulação dos dados realizadas pelas operações analíticas. O cubo de dados do exemplo da cadeia de lojas é ilustrado na Figura 2.1. Produto, filial e tempo são dimensões e o valor da medida numérica, unidades vendidas, é representado em cada célula. A multidimensionalidade intrínseca das requisições realizadas pelos usuários de SSD torna a modelagem multidimensional apropriada para DWs.

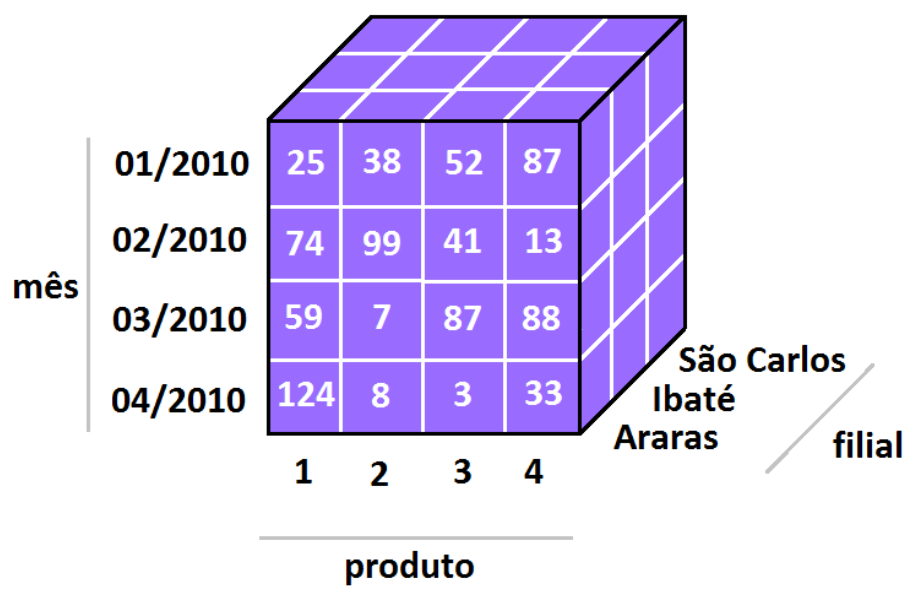

Figura 2.1: Cubo de dados multidimensional do exemplo da cadeia de lojas.

\subsubsection{Níveis de Agregação}

Em geral, DWs são organizados em níveis de agregação, desde um nível inferior que possui dados mais detalhados, até um nível superior que possui dados muito resumidos. Também podem existir vários níveis intermediários entre os níveis superior e inferior, representando graus crescentes de agregação (Agrawal et al., 2000; Baralis et al., 1997; Derakhshan et al., 2008; Gupta e Mumick, 1999; Harinarayan et al., 1996; Hung et al., 2007; Kotidis e Roussopoulos, 2001; Lawrence e Rau-Chaplin, 2006; Shah et al., 2006).

Cada nível de agregação de um DW pode ser considerado uma visão, sendo que os relacionamentos entre as diversas visões de um cubo de dados multidimensional podem ser representados por um lattice (Harinarayan et al., 1996), o qual é também conhecido como 
grafo de derivação. Um grafo de derivação é composto por um conjunto de visões $\mathcal{L}$ e uma relação de dependência $\preceq$. Sejam $\mathcal{V}_{1}$ e $\mathcal{V}_{2}$ duas visões. Tem-se que $\mathcal{V}_{1} \preceq \mathcal{V}_{2}$ se $\mathcal{V}_{1}$ pode ser derivada a partir de $\mathcal{V}_{2}$. Logo, $\mathcal{V}_{1}$ é descendente de $\mathcal{V}_{2}$ e $\mathcal{V}_{2}$ é ancestral de $\mathcal{V}_{1}$. Toda visão é ancestral e descendente dela mesma. O grafo de derivação é um grafo direcionado, no qual ancestrais apontam para seus descendentes.

O grafo de derivação das agregações das dimensões produto $p$, filial $f$ e tempo $t$ do cubo de dados da cadeia de lojas é ilustrado na Figura 2.2. Nesse exemplo, a visão $p f$ é um ancestral direto de $p$ e $f$ e representa os dados agregados por produto e filial. A visão vazio contém o nível mais agregado de dados e é descendente de todas as visões. A visão pft possui os dados mais detalhados e é ancestral de todas as outras visões do grafo.

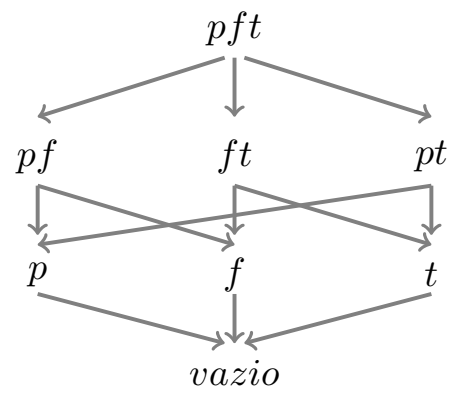

Figura 2.2: Grafo de derivação das dimensões do exemplo da cadeia de lojas.

\subsubsection{Visão Materializada}

As visões representadas no grafo de derivação podem ser materializadas, ou seja, elas podem ser tabelas armazenadas fisicamente no DW contendo dados mais agregados que seus ancestrais. A materialização de visões é um método bastante usado para melhorar o desempenho de consultas em DWs (Agrawal et al., 2000; Baikousi e Vassiliadis, 2009; Hung et al., 2007; Kotidis e Roussopoulos, 2001), desde que, ao invés da resposta ser calculada em tempo de execução, ela é pré-armazenada no DW. Com isso, evita-se a necessidade de se realizar operações custosas que envolvam group by e junções de tabelas de dimensão.

O grande número de dimensões e hierarquias de atributos existentes em um DW torna impraticável a materialização de todas as visões existentes em um grafo de derivação. Isso pode gerar um alto custo de armazenamento e manutenção de todas essas visões. Diversas estratégias e algoritmos existentes na literatura (Baralis et al., 1997; Harinarayan et al., 1996) podem ser utilizados para uma boa escolha de quais visões materializar.

\subsubsection{Representação Lógica dos Dados}

A modelagem multidimensional por meio de dimensões e medidas numéricas representa o modelo conceitual dos dados. Para a modelagem lógica dos dados são utilizadas duas abordagens: 
sistemas ROLAP (OLAP Relacional) e sistemas MOLAP (OLAP Multidimensional). Sistemas ROLAP armazenam os dados em sistemas de gerenciamento de banco de dados relacionais (SGBDRs). Já sistemas MOLAP possuem estruturas de dados especializadas, geralmente matriciais e as operações OLAP (seção 2.1.5) são implementadas diretamente no cubo de dados. Nesta dissertação, são utilizados DWGs implementados em SGBDRs, portanto o enfoque é em sistemas ROLAP.

Sistemas ROLAP utilizam relações (tabelas) para armazenar os dados em conjunto com uma camada de software que implementa as operações OLAP. Em bancos de dados relacionais, dimensões e medidas numéricas são implementadas por meio de dois esquemas: esquema estrela e esquema floco de neve (Kimball e Ross, 2002).

O esquema estrela consiste em uma tabela de fatos central cercada por várias tabelas de dimensão. Seu nome é originário do fato do desenho formado pelo esquema se assemelhar a uma estrela. A tabela de fatos armazena as medidas numéricas e referências para as dimensões correspondentes, as quais são feitas por meio de chaves estrangeiras que compõem a chave primária da tabela de fatos. As tabelas de dimensão armazenam dados descritivos sobre as dimensões que contextualizam as medidas numéricas. A tabela de fatos é caracterizada por possuir um número pequeno de colunas e um grande número de linhas, enquanto que tabelas de dimensão possuem muitas colunas e poucas linhas. Na Figura 2.3 é ilustrado o esquema estrela do Star Schema Benchmark (SSB) ${ }^{1}$, composto por uma tabela de fatos lineorder que armazena várias medidas numéricas (e.g., discount e supplycost) e pelas tabelas de dimensão supplier, customer, part e date.

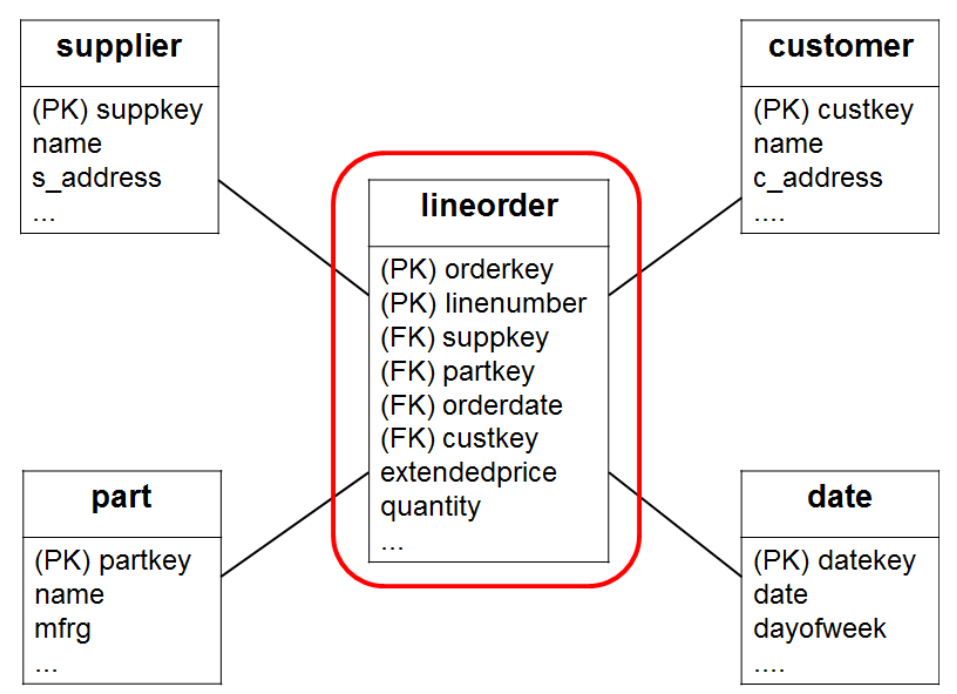

Figura 2.3: Esquema estrela do SSB.

O esquema floco de neve é gerado a partir da normalização das tabelas de dimensão do esquema estrela por meio das hierarquias de atributos. Dessa maneira, tabelas

\footnotetext{
${ }^{1}$ P. O'Neil, E. O'Neil, X. Chen. http://www.cs.umb.edu/ poneil/StarSchemaB.PDF.
} 
de dimensão passam a estar ligadas a outras tabelas de dimensão. A normalização da dimensão customer do esquema estrela do SSB feito a partir da hierarquia de atributos (region_name) $\preceq$ (nation_name) $\preceq$ (city_name) $\preceq$ (c_address) é ilustrada na Figura 2.4.

\begin{tabular}{|c|c|c|c|}
\hline customer & city & nation & region \\
\hline $\begin{array}{l}\text { (PK) custkey } \\
\text { name }\end{array}$ & $\begin{array}{l}\text { (PK) citykey } \\
\text { city_name }\end{array}$ & (PK) nationkey & $\begin{array}{l}\text { (PK) regionkey } \\
\text { region_name }\end{array}$ \\
\hline $\begin{array}{l}\text { c_address } \\
\text { (FK) citykey } \\
\ldots\end{array}$ & (FK) nationkey & (FK) regionkey & \\
\hline
\end{tabular}

Figura 2.4: Normalização da dimensão customer do SSB.

A normalização realizada pelo esquema floco de neve pode propiciar um custo menor no espaço de armazenamento, pois há uma diminuição da redundância dos dados. No entanto, no contexto de DW, o ponto crucial é o desempenho de consultas, e a redundância dos dados gerada pelo esquema estrela em geral tende a ser favorável. Isso acontece porque um número menor de junções entre tabelas é realizado para responder às consultas. Uma possível desvantagem é o custo da manutenção de consistência entre os dados redundantes.

A união de diferentes esquemas com dimensões em comum pode originar constelações de fatos. Essas constelações são formadas quando tabelas de fatos diferentes compartilham uma ou mais tabelas de dimensão. A Figura 2.5 ilustra uma constelação de fatos extraída do benchmark TPC-H (Pöss e Floyd, 2000). Esse benchmark modela uma aplicação de DW que representa dados históricos relativos a pedidos e vendas de uma corporação. Part, Orders e Supplier são tabelas de dimensão, enquanto que Lineitem e Partsupp são tabelas de fatos. As medidas numéricas de Lineitem são extendedprice e discount, e as medidas numéricas de Partsupp são availqty e supplycost.

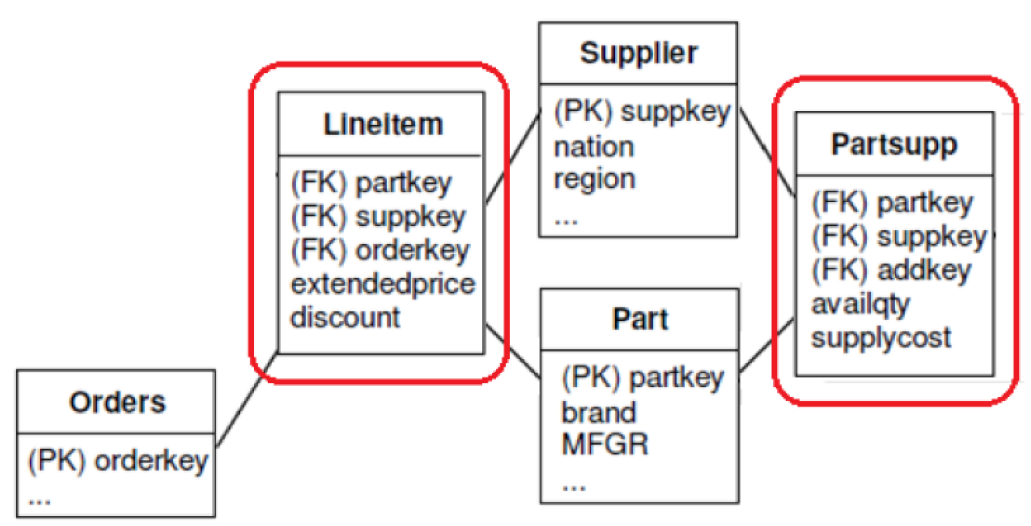

Figura 2.5: Constelação de fatos extraída do benchmark TPC-H. 


\subsubsection{Operações OLAP}

O modelo multidimensional em conjunto com o uso de diferentes níveis de agregação permite a realização das operações analíticas, dentre as quais se destacam: drill-down, roll-up, slice and dice, pivot e drill-across.

A operação drill-down consiste em realizar análises cada vez mais detalhadas, ou seja, em níveis cada vez menores de granularidade. Por exemplo, um usuário de SSD pode requisitar o total de unidades vendidas por produto por filial por ano, em seguida requisitar o total de unidades vendidas por produto por filial por mês e por fim, o total de unidades vendidas por produto por filial por dia. Esse tipo de análise é importante para que, a partir de uma análise geral, possam identificar-se falhas pontuais no processo de negócio de uma organização.

Inversa à drill-down é a operação roll-up. Nela são realizadas análises em grãos cada vez maiores, ou seja, menos detalhados. Nesse caso, um usuário de SSD segue a sequência inversa à anterior. A primeira requisição é o total de unidades vendidas por produto por filial por dia, em seguida, o total de unidades vendidas por produto por filial por mês e por fim, o total de unidades vendidas por produto por filial por ano. Isso proporciona uma visão cada vez mais ampla do negócio. As operações drill-down e roll-up são ilustradas na Figura 2.6.
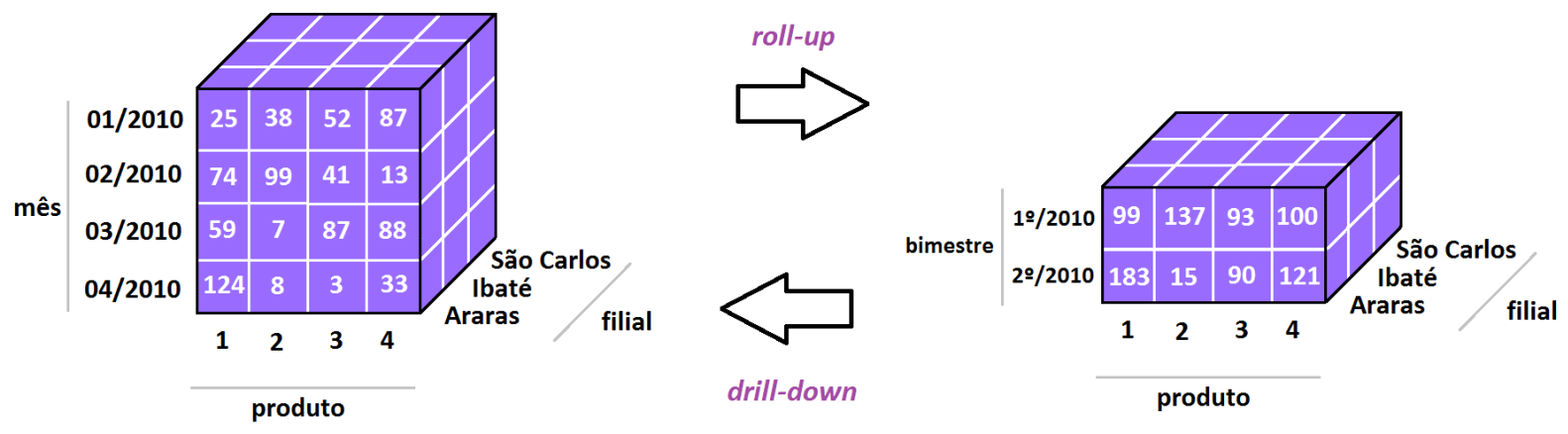

Figura 2.6: Exemplo de operações drill-down e roll-up.

A operação pivot corresponde a uma mudança de perspectiva em uma análise. Essa operação altera a ordem de visualização dos dados e corresponde a um giro do cubo de dados. Por exemplo, um usuário de SSD pode requisitar o total de unidades vendidas por produto por filial por mês e, em uma nova análise, requisitar o total de unidades vendidas por mês, por filial e por produto. Essa operação é ilustrada na Figura 2.7.

A operação slice and dice permite que os usuários realizem cortes no cubo de dados multidimensional. Desta maneira, há uma restrição em algumas dimensões para um valor fixo (slice) ou para uma faixa de valores (dice). O exemplo ilustrado na Figura 2.8 mostra uma operação slice quando o valor de filial é fixado em Araras e uma operação dice quando é feita a seleção da faixa 03/2010 à 04/2010 na dimensão mês.

Por fim, a operação drill-across, que é um dos dois tipos de consultas OLAP contempladas nesta dissertação, realiza análises que envolvam medidas numéricas diferentes que possuam 

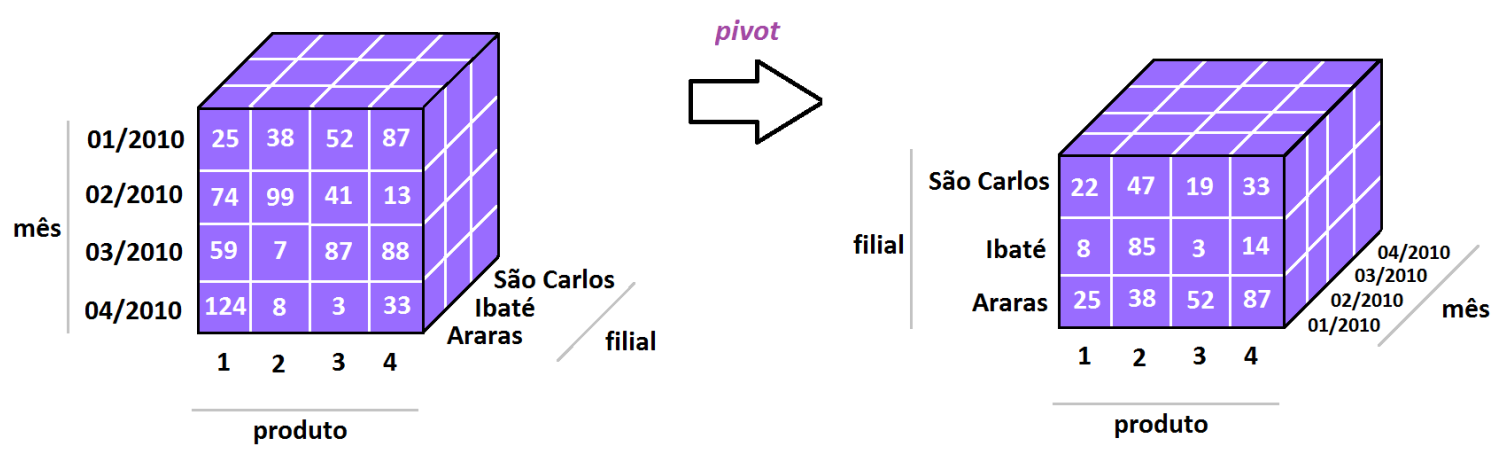

Figura 2.7: Exemplo de operação pivot.
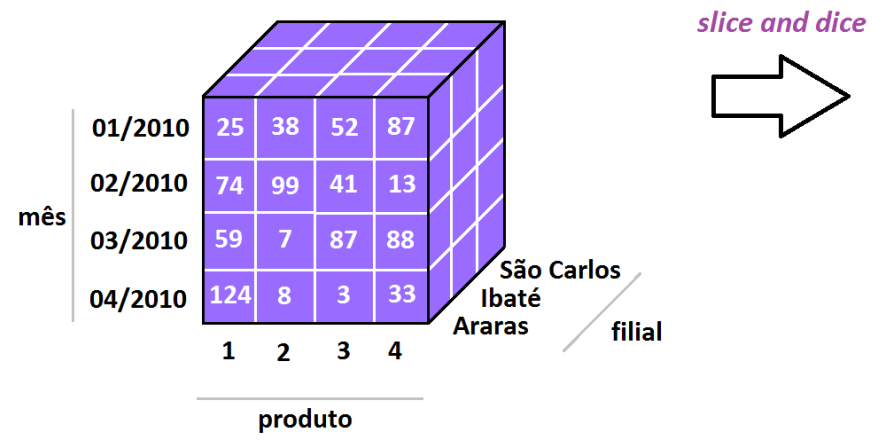

Figura 2.8: Exemplo de operação slice and dice.

pelo menos uma dimensão em comum. Essas dimensões devem ser compartilhadas, ou seja, exatamente iguais. Além disso, deve haver uma compatibilidade na granularidade utilizada em cada uma das medidas numéricas para que elas possam ser analisadas em conjunto. Quando essas medidas não estão na mesma granularidade, agregações devem ser realizadas para que as operações drill-across sejam ser processadas.

Seja o esquema representado na Figura 2.5. Exemplos de consultas que podem ser realizadas contra um DW projetado de acordo com esse esquema são:

- "Liste extendedprice, discount, availqty, supplycost por brand por nation".

- 'Liste extendedprice, discount, availqty, supplycost por nation'.

Essas consultas representam consultas drill-across porque recuperam as medidas numéricas extendedprice e discount da tabela de fatos Lineitem e as medidas numéricas availqty e supplycost da tabela de fatos Partsupp, relacionando-as por meio das tabelas de dimensão compartilhadas Part e Supplier. 


\subsection{Data Warehouse Geográfico}

Diferentemente de um DW convencional, um DWG armazena dados espaciais como atributos específicos em tabelas de dimensão ou como medidas em tabelas de fatos (Bimonte et al., 2005; Fidalgo et al., 2004; Malinowski e Zimányi, 2004, 2005; Sampaio et al., 2006; Silva et al., 2006, 2008; Stefanovic et al., 2000). Adicionalmente, hierarquias de atributos também podem ser definidas para os atributos espaciais. Dois tipos de hierarquias espaciais são considerados na literatura: ad hoc e predefinida. Uma hierarquia espacial ad hoc associa objetos espaciais de diferentes granularidades por meio do agrupamento desses objetos usando um índice espacial (e.g., R-tree), o qual indica a forma na qual as medidas numéricas correspondentes são agregadas (Papadias et al., 2001). Já uma hierarquia espacial predefinida é uma associação 1:N entre atributos espaciais de mais alta e mais baixa granularidade, a qual é determinada por um relacionamento topológico (e.g., intersecta, contém, está contido) (Malinowski e Zimányi, 2005). Por exemplo, uma cidade (polígono) contém vários endereços (pontos) e um endereço está contido em uma única cidade.

A Figura 2.9 ilustra um esquema híbrido de DWG, o New Geographic Hybrid Star Schema Benchmark (NewGHSSB) (Mateus et al., 2010), derivado do benchmark SSB ${ }^{1}$. Esse esquema difere-se de um esquema estrela convencional porque ele inclui tabelas de dimensão e dados espaciais. Part e date são tabelas de dimensão convencionais que armazenam somente dados redundantes e descritivos, enquanto que customer e supplier, city, nation e region são tabelas de dimensão espaciais. Nas tabelas de dimensão espaciais, atributos com o sufixo _geo, como city_geo, são atributos espaciais que armazenam geometrias. Note que os esquemas híbrido e floco de neve são diferentes, desde que o primeiro não normaliza as hierarquias de atributos espaciais.

Existem duas hierarquias de atributos espaciais no esquema do DWG ilustrado na Figura 2.9: $(i)($ region_geo $) \preceq($ nation_geo $) \preceq($ city_geo $) \preceq($ c_address_geo $)$; e $($ ii $)($ region_geo $) \preceq$ (nation_geo $) \preceq($ city_geo $) \preceq($ s_address_geo $)$. Essas hierarquias são classificadas como hierarquias espaciais predefinidas do tipo simétrico simples com o relacionamento topológico está contido, especificando que uma geometria em um atributo de granularidade espacial de nível mais baixo está contida em somente uma geometria em um atributo de granularidade espacial de nível mais alto (Malinowski e Zimányi, 2005).

Nas seções 2.2.1 e 2.2.2 são discutidos tipos de dados espaciais e consultas SOLAP, respectivamente.

\subsubsection{Tipos de Dados Espaciais}

Os tipos de dados espaciais mais básicos e comumente encontrados em SGBDRs espaciais são ponto, linha e polígono. Ponto é a entidade mais primitiva para representar objetos espaciais. Um objeto espacial representado por um único ponto é dito sem dimensionalidade 


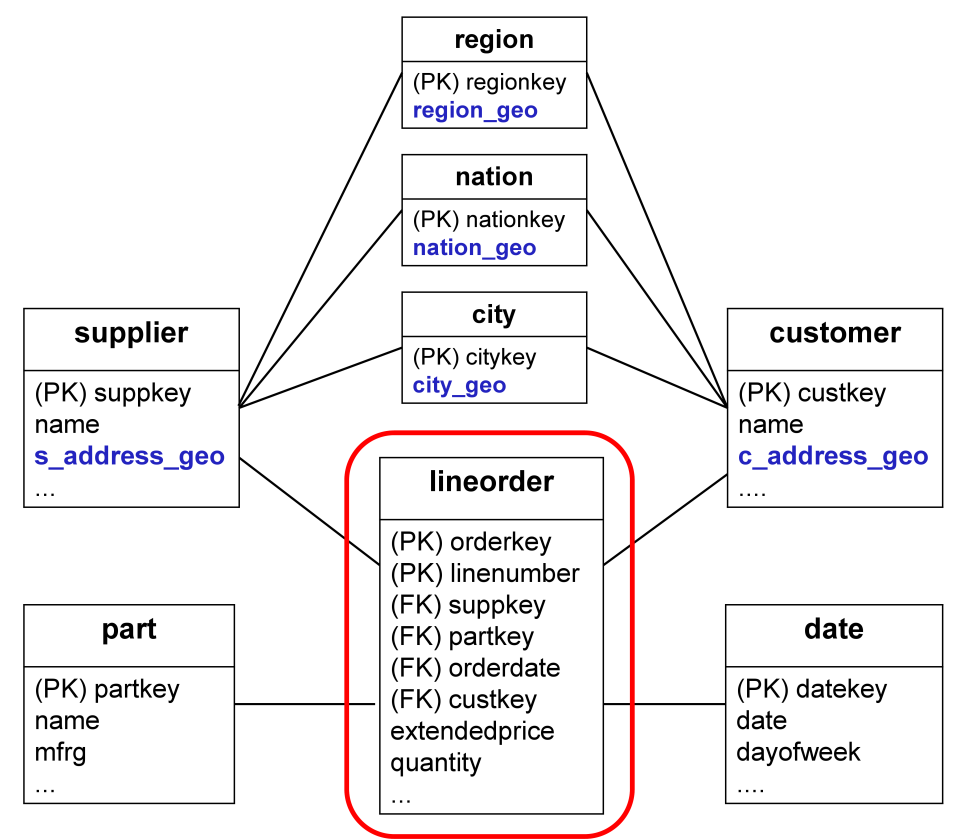

Figura 2.9: New Geographic Hybrid SSB (NewGHSSB).

ou 0-dimensional (e.g., um carro em uma estrada, uma casa). Uma sequência de pontos conectados forma uma linha e essa é uma estrutura 1-dimensional. Polígono é uma sequência de linhas conectadas, cujo primeiro ponto coincide com o último e é dito ser 2-dimensional. Além desses tipos de dados espaciais básicos ilustrados na Figura 2.10, outros tipos de dados espaciais são ilustrados em (Ciferri, 2002), como exemplo polígono complexo com buraco, polígono complexo com ilha e poliedro.

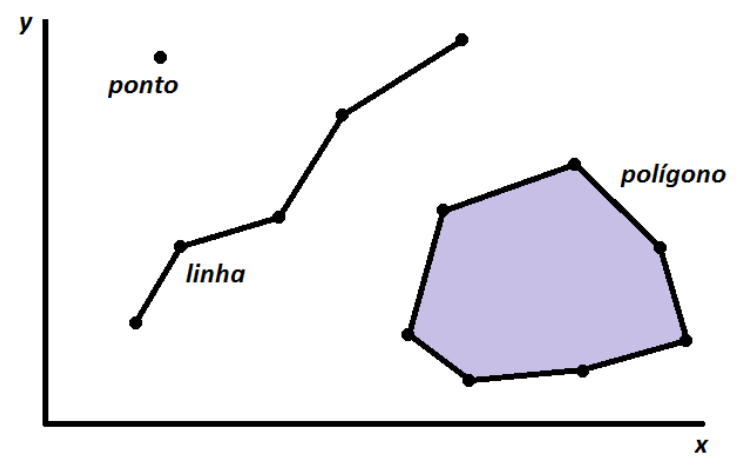

Figura 2.10: Exemplos de tipos de dados espaciais.

A análise de um predicado espacial pode se tornar extremamente custosa, pois objetos espaciais podem conter da ordem de milhares de pontos. Por exemplo, pode-se citar um polígono representando com precisão a geometria de uma cidade que possua uma fronteira 
muito acidentada. Dessa maneira, uma consulta por um relacionamento topológico (e.g., intersecta, contém, está contido) sobre dados espaciais é realizada em duas fases.

A primeira fase é chamada de filtragem, na qual um índice é utilizado para reduzir o conjunto de dados a ser analisado. Nessa fase, uma representação aproximada do objeto espacial é utilizada, por exemplo, o minimun bounding rectangle (MBR). Os objetos espaciais selecionados nessa fase são chamados de candidatos. Uma segunda análise é necessária, pois a utilização de representações aproximadas pode selecionar falsos candidatos.

A segunda fase é chamada de refinamento, na qual os objetos espaciais candidatos selecionados na fase anterior são avaliados por sua geometria exata. Os falsos candidatos são descartados e os objetos que satisfazem o relacionamento topológico considerado são selecionados.

O processamento em duas fases proporciona um ganho de desempenho significativo, pois poucos objetos têm que passar pela fase de refinamento, que é a fase mais cara. Na verdade, pode ser que haja mais de uma fase de filtragem, ou seja, filtragens que utilizem aproximações melhores progressivamente.

\subsubsection{Consultas SOLAP}

Consultas SOLAP são consultas que realizam operações OLAP (seção 2.1.5) e possuem predicados espaciais sobre dados espaciais. Consultas com predicados espaciais são geralmente classificadas de acordo com esse predicado. São exemplos dessas classificações: point queries, region queries, range queries e consultas com junção espacial. Nas seções 2.2.2.1 e 2.2.2.2 são resumidas as consultas do tipo range queries e consultas com junção espacial, respectivamente, por se tratarem de tipos considerados pelas estratégias de processamento propostas nesta dissertação. Os predicados das consultas drill-across usadas nesta dissertação são do tipo range queries. Já o processamento de consultas SOLAP com junção espacial é um dos enfoques desta dissertação.

\subsubsection{Range Queries}

Esse tipo de consulta busca por objetos espaciais que satisfaçam o predicado espacial de acordo com um intervalo (range), o qual é definido por uma janela de consulta, geralmente quadrada. De acordo com o predicado espacial, as range queries são classificadas nos seguintes subtipos:

(a) Intersection range query: seleciona todos os objetos espaciais que compartilhem ao menos um ponto com a janela de consulta (Figura 2.11.a).

(b) Containment range query: seleciona todos os objetos espaciais que estejam contidos na janela de consulta (Figura 2.11.b).

(c) Enclousure range query: seleciona os objetos espaciais nos quais a janela de consulta está contida (Figura 2.11.c). 


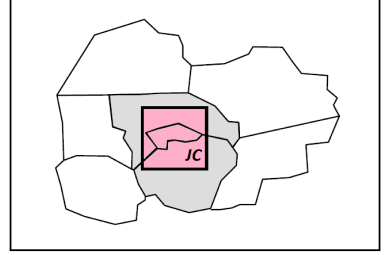

(a) Intersection range query.

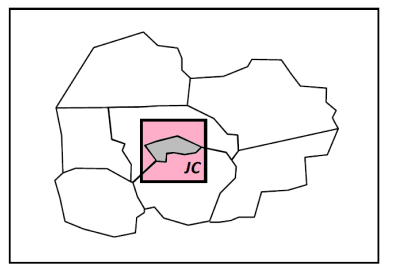

(b) Containment range query.

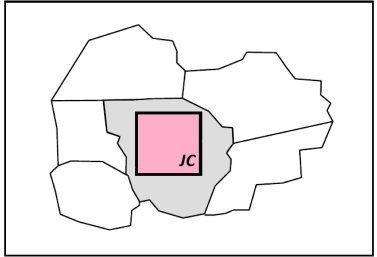

(c) Enclousure range query.

Figura 2.11: Subtipos de range queries - adaptada de (Ciferri, 2002b).

\subsubsection{Consultas com junção espacial}

Consultas com junção espacial ou spatial join queries são consultas que realizam operações de junção espacial. A operação de junção espacial combina dois conjuntos de dados espaciais de acordo com um predicado espacial. Em outras palavras, a operação de junção espacial seleciona um subconjunto do produto cartesiano de dois conjuntos de objetos espaciais de acordo com um relacionamento topológico entre esses objetos. Consultas com junção espacial formam um conjunto importante de consultas devido à sua grande aplicabilidade em análises realizadas em sistemas de informações geográficas (SIGs) e em bancos de dados espaciais. Por exemplo, operações de junção espacial podem ser aplicadas na execução de map overlay, que é uma espécie de sobreposição de mapas.

Exemplos de consultas SOLAP com junção espacial são: "Liste o total de unidades vendidas por produto, por filial, por mês em filiais cujas cidades são intersectadas por rios", "Liste o lucro total por cliente, por produto para clientes que estão localizados a menos de $100 \mathrm{Km}$ de distância do fornecedor" e "Liste o total de unidades vendidas por produto, por fornecedor considerando vendas para clientes cujas cidades são vizinhas da cidade do fornecedor". O último exemplo realiza uma operação de junção entre as cidades dos clientes e as cidades dos fornecedores para verificar quais são vizinhas. Vários operadores espaciais podem ser empregados para compor o predicado, sendo o de intersecção o mais comum.

Em geral, o processamento de uma junção espacial também é realizado em duas fases: filtragem e refinamento. A fase de filtragem seleciona pares de objetos espaciais candidatos representados por meio de uma aproximação do objeto original, por exemplo, MBR. A fase de refinamento avalia o predicado espacial utilizando a geometria exata dos pares candidatos e seleciona os pares que satisfaçam o predicado.

Sejam $A$ e $B$ duas relações que armazenam dados espaciais representados por polígonos, sendo $m$ e $n$ o número de objetos espaciais em cada relação respectivamente. Uma consulta com junção espacial entre as relações $A$ e $B$ utilizando o predicado intersecta seleciona pares de objetos espaciais representados por $\left(a_{i}, b_{j}\right)$ onde $1 \leq i \leq m, 1 \leq j \leq n$ e $a_{i}$ e $b_{j}$ se intersectam. Essa consulta gera uma combinação de $m \times n$ pares de objetos espaciais. Para 
relações com um grande número de objetos espaciais, esse valor pode se tornar extremamente alto, tornando indispensável o uso de algoritmos que realizem junções espaciais eficientemente.

Segundo Gaede e Günther (1998), não é possível determinar uma ordenação total sobre objetos espaciais que preserve a proximidade desses objetos. Consequentemente, a aplicação de algoritmos conhecidos que realizam junções convencionais (e.g., merge-join) não são aplicáveis a junções espaciais. Isso se reflete em um grande número de trabalhos voltados ao estudo e desenvolvimento de algoritmos de junção espacial. Este assunto será abordado mais detalhadamente na seção 3.1.3.

\subsection{Considerações Finais}

Neste capítulo foram discutidos os principais conceitos relacionados a DWs convencionais e geográficos, dentre os quais se destacam: (i) a modelagem multidimensional, por meio da descrição de dimensões e medidas numéricas; (ii) a representação lógica dos dados, a qual define a maneira na qual os dados são armazenados fisicamente nos SGBDRs; (iii) o uso de visões materializadas como uma forma de melhorar o desempenho das consultas; (iv) as operações OLAP que determinam as possíveis manipulações que os dados em um DW podem sofrer; (v) os principais tipos de dados espaciais presentes nos DWGs; e (vi) os principais tipos de consultas SOLAP que são de interesse em DWGs.

A integração dos dados provenientes dos SIGs, DW e OLAP deram origem aos DWGs e às consultas SOLAP. Isso trouxe um diferencial para as análises efetuadas pelos usuários de SSD, pois atualmente muitas as informações são georreferenciadas. Estratégias podem ser traçadas com base no posicionamento das informações, como exemplo: "Qual o volume de vendas de cada produto para clientes em um raio de $100 \mathrm{Km}$ ?".

Embora alguns aspectos que influenciem a busca de desempenho no processamento das consultas que incidam sobre DWs convencionais e geográficos tenham sido destacados neste capítulo, não foram discutidos índices para esses dados. O próximo capítulo trata justamente esse assunto: índices. 

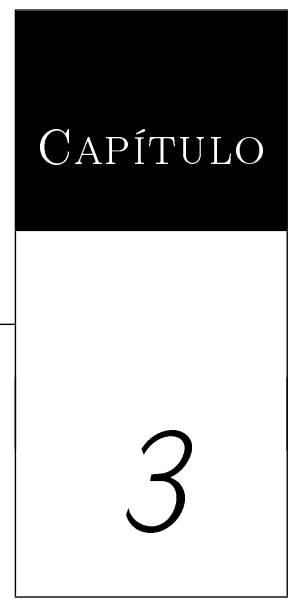

Índices

Índices são estruturas de dados projetadas para atuarem como um caminho otimizado aos dados. Um índice organiza o espaço indexado de tal forma que a recuperação dos dados que satisfaçam uma consulta não requeira a análise do conjunto completo dos dados armazenados. Um índice é definido por uma estrutura de dados, e de algoritmos de busca e alteração de sua estrutura. Neste capítulo são descritos os principais índices espaciais (seção 3.1) e para DWs convencionais (seção 3.2) existentes na literatura. O capítulo é finalizado na seção 3.3, com as considerações finais.
\end{abstract}

\title{
3.1 Índices Espaciais
}

Um índice espacial é voltado à indexação de objetos espaciais. A organização mais utilizada é uma estrutura hierárquica na forma de uma árvore de múltiplos caminhos ou na forma de uma estrutura hash, ou ainda uma combinação entre elas (Ciferri, 2002). A R-Tree e a R*-Tree, dois dos índices espaciais mais referenciados na literatura, são descritos nas seção 3.1.1. A seção 3.1.3 discute as principais características de algoritmos de junção espacial.

\subsubsection{R-Tree}

A R-Tree (Gaede e Günther, 1998; Guttman, 1984) é uma estrutura de indexação espacial utilizada em dados multidimensionais. Em sua estrutura, objetos espaciais são representados 
por meio de intervalos em suas dimensões. No contexto desta dissertação, indexação de DWGs, o espaço dos objetos espaciais considerados (pontos, linhas e polígonos) é bidimensional e por isso são representados por meio de seu MBR. O MBR de um objeto bidimensional (Figura 3.1) é definido por suas quatro coordenadas $x_{\min }, y_{\min }, x_{\max }$ e $y_{\max }$. A área definida pela parte externa do objeto e que está contida no seu MBR é chamada de deadspace.

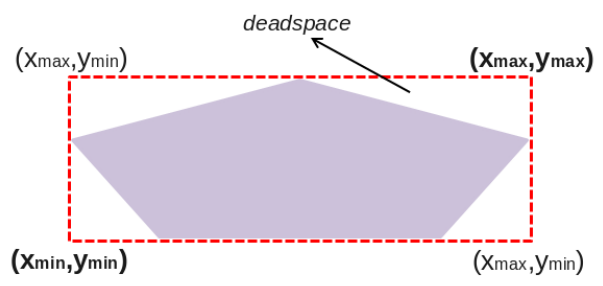

Figura 3.1: Minimum Bounding Rectangle (MBR) de um objeto espacial.

Ilustrada na Figura 3.2, a R-Tree constrói uma hierarquia baseada no MBR dos objetos espaciais. Existem dois tipos de nós: interno e folha. Nós internos possuem entradas que armazenam um ponteiro para outro nó (nó filho), e o MBR que engloba o MBR de todas as entradas de seu nó filho. Nós folhas possuem entradas que armazenam o MBR e um ponteiro para o objeto espacial. Uma estrutura no formato de árvore balanceada é mantida de forma que todos os nós folhas encontram-se no mesmo nível. Cada nó possui de $m$ a $M$ entradas, onde $m \leq M / 2$. O valor $M$ pode ser ajustado de acordo com o desempenho desejado. Somente o nó raiz pode possuir menos de $m$ entradas.

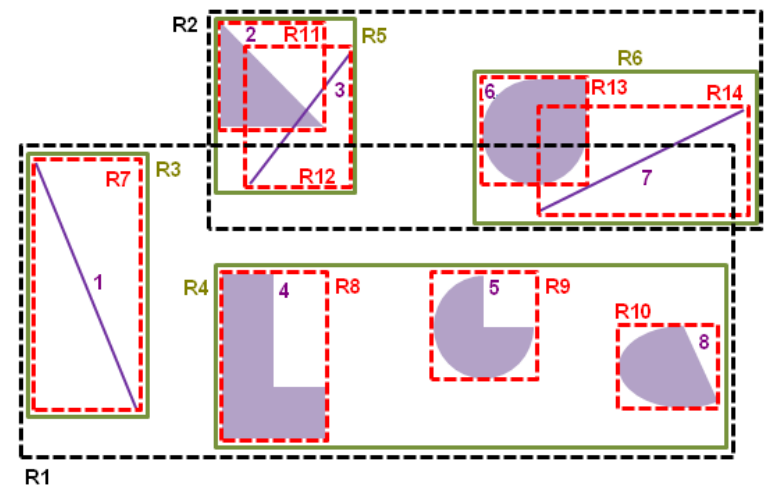

(a) Particionamento espacial da R-Tree.

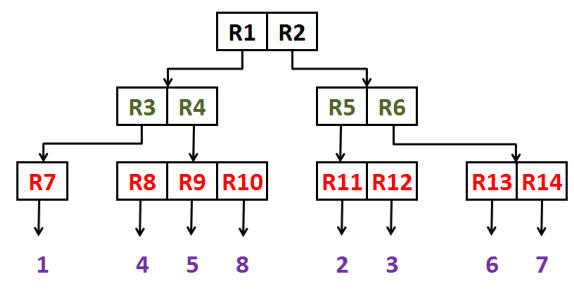

(b) Estrutura hierárquica da R-Tree.

Figura 3.2: Exemplo de R-Tree.

No exemplo da Figura 3.2, os objetos espaciais estão numerados de 1 a 8 e os MBRs identificados por $R_{i}$, onde $1 \leq i \leq 14$. Cores identificam os diferentes níveis formados pela hierarquia dos MBRs. Nesse exemplo, é possível identificar as intersecções entre MBRs de um mesmo nível. Nesse caso, é dito que os nós estão indexando a mesma porção do espaço. Além dessa definição da estrutura de dados da R-Tree, a seção 3.1.1.1 detalha a inserção de 
novas entradas na árvore, enquanto a seção 3.1.1.2 descreve as características dos algoritmos de busca.

\subsubsection{Inserção de novas entradas}

Novas entradas são inseridas em nós folhas e, se necessária, é realizada a promoção de novos nós até a raiz. Partindo de uma árvore vazia, novas entradas são inseridas no nó raiz até que sua capacidade máxima $M$ seja atingida. Uma nova tentativa de inserção nessa raiz causará uma divisão desse nó em dois e a criação de um novo nó raiz. As entradas do novo nó raiz apontarão para os dois nós resultantes da divisão.

Quando a árvore já possui mais de um nível, novos dados são inseridos em nós folhas. Caso haja espaço, a entrada é simplesmente inserida em um nó folha. Se a inserção de uma nova entrada deve ser feita em um nó que está cheio, esse nó sofre uma divisão. O nó é dividido em dois, ou seja, um novo nó é criado e metade das entradas do antigo nó são transferidas para o novo nó. Uma nova entrada é criada no nível superior e aponta para o nó recém criado. $O$ novo dado é então inserido. Esse processo é repetido sempre que uma entrada causa o estouro da capacidade de um nó. Além disso, toda vez que uma nova entrada é inserida na árvore, é preciso realizar uma atualização do MBR. Essa atualização deve ser propagada aos níveis superiores até que a alteração do MBR de uma entrada de um nível $l$ não afete o MBR de uma entrada do nível $l-1$.

A distribuição das entradas em uma divisão e a escolha dos nós em que novas entradas são inseridas visa sempre minimizar a área total ocupada pelos MBRs de um nó. Em seu trabalho, Guttman (1984) propõe alguns algoritmos de particionamento do espaço com esse objetivo.

\subsubsection{Algoritmo de busca}

O algoritmo de busca mais básico da R-Tree utiliza uma consulta do tipo intersection range query (Figura 2.11.a). Em um percurso top-down pela árvore a partir da raiz, cada entrada tem seu MBR avaliado. É verificado se o MBR da entrada intersecta a janela de consulta. Caso o predicado seja de junção espacial, essa janela é substituída pelo MBR de outro objeto espacial. Nós filhos de entradas cujo MBR não satisfaz a consulta são descartados. Dessa maneira, é realizada uma poda na árvore. Esse é o maior benefício da estrutura hierárquica da R-Tree, pois essa poda faz com que muitos nós não sejam visitados, diminuindo o número de acessos a disco e cálculos desnecessários. Em contrapartida, nós filhos de entradas cujos MBRs intersectam a janela (ou o MBR) são avaliados, gerando uma ramificação do percurso, a qual pode degenerar o desempenho do índice, fazendo com que um número grande de nós sejam visitados e, consequentemente, um grande número de páginas de disco sejam recuperadas.

Se a entrada de um nó folha é avaliada positivamente, o objeto indexado por ela tem sua geometria exata avaliada ou é enviado diretamente para a resposta. Essa avaliação considerando a forma exata é chamada de fase de refinamento. A R-Tree é percorrida até que não 
haja mais entradas para serem avaliadas. Esse envio direto pode acontecer dependendo do tipo de dado e predicado utilizado, por exemplo, um ponto é completamente definido por seu MBR, fazendo com que uma fase de refinamento seja dispensada em uma range query com o predicado está contido.

A utilização de uma representação aproximada (MBR) faz com que objetos que não satisfaçam o predicado espacial sejam selecionados. Esses são chamados de falsos candidatos. Quanto melhor for a aproximação utilizada, menos falsos candidatos são selecionados. Por outro lado, o custo de armazenamento de uma aproximação melhor é provavelmente mais caro, assim como o custo de verificação das entradas no processo de busca.

\subsubsection{R*-Tree}

Segundo Gaede e Günther (1998), a R*-Tree (Beckmann et al., 1990) é uma proposta baseada em uma série de estudos de desempenho da R-Tree sob diferentes configurações da distribuição dos dados espaciais. Por meio desse estudo, Beckmann et al. (1990) introduziram uma política chamada reinserção forçada. Quando um nó estoura sua capacidade, ao invés de dividir o nó em dois da mesma forma que a R-Tree, essa política determina que $p$ entradas devem ser retiradas desse nó e reinseridas na árvore. Em seu trabalho, Beckmann et al. (1990) sugerem que o valor de $p$ seja em torno de $30 \%$ do total de entradas do nó.

Outra diferença diz respeito ao particionamento do espaço. A R*-Tree tenta particionar o espaço de forma que a área total dos MBRs de um nó seja mínima. Além desse, a $\mathrm{R}^{*}$-Tree tem como objetivos:

- Minimizar a sobreposição dos MBRs de entradas de nós do mesmo nível.

- Minimizar o perímetro dos MBRs de entradas de nós internos.

- Maximizar a utilização do espaço de armazenamento.

Além disso, Beckmann et al. (1990) propõem um algoritmo de divisão dos nós de complexidade $O(n d \log n)$, onde $n$ é o número de entradas do nó e $d$ é a dimensão do espaço. A essência dos algoritmos de busca e exclusão de dados é a mesma dos da R-Tree. Roussopoulos e Leifker (1985) já haviam observado que a inserção dos dados era o ponto crítico da R-Tree, pois determina o desempenho do algoritmo de busca e, em seus estudos, Beckmann et al. (1990) comprovaram esse fato.

\subsubsection{Algoritmos de Junção Espacial}

Segundo Brinkhoff et al. (1993), as consultas espaciais podem ser classificadas em single scan queries e multiple scan queries. As single scan queries são consultas em que os objetos espaciais são percorridos uma única vez (e.g., range queries, point queries), enquanto que 
multiple scan queries são consultas em que os objetos espaciais necessitam ser percorridos mais de uma vez. Consultas de junção espacial fazem parte desse segundo grupo.

Para as single scan queries, um processamento eficiente é atingido com o uso de diversos índices espaciais conhecidos (e.g., R-Tree). Já para as multiple scan queries, novas estratégias foram desenvolvidas. Essa estratégias são em sua maioria baseadas em algoritmos aplicados em junções convencionais e índices espaciais utilizados em single scan queries, sendo os da família R-Tree os mais utilizados.

De acordo com Papadopoulos et al. (1999), os algoritmos de junção espacial podem ser classificados a partir da disponibilidade de índices espaciais nas relações envolvidas. Essa classificação é realizada da seguinte maneira:

- Algoritmos para relações não indexadas.

- Algoritmos para relações indexada e não indexada.

- Algoritmos para relações indexadas.

Cada um desses tipos de algoritmos se baseia em diferentes estratégias para o cálculo da junção espacial. Günther et al. (1999) fazem uma breve descrição dessas estratégias. Para algoritmos de junção espacial entre relações não indexadas, a estratégia mais utilizada é baseada no algoritmo Nested Loop, o qual, utilizado em junções convencionais, possui a estratégia mais simples e direta. Ela consiste em dois laços aninhados que percorrem as duas relações sequencialmente, testando todos os possíveis pares de objetos espaciais por meio de sua geometria exata. Em geral, essa estratégia tem o pior desempenho no processamento das consultas devido ao grande número de testes necessários.

Quando uma das duas relações envolvidas na junção espacial dispõe de um índice espacial, a estratégia escolhida é chamada de Scan-and-Index, a qual consiste em uma mistura de busca sequencial em uma relação e busca indexada na outra relação. Cada entrada lida sequencialmente em uma relação busca por seus pares utilizando o índice da outra relação. Sua vantagem com relação à estratégia anterior é que nem todos os objetos espaciais da relação indexada são testados pelo predicado espacial.

O último tipo, algoritmos para relações indexadas, é o mais encontrado na literatura, visto que a maioria dos SGBDRs dispõe de índices espaciais. A estratégia mais utilizada para esse tipo de algoritmo é chamada de Synchronized Tree Traversal. Ela consiste de um percurso sincronizado entre duas estruturas hierárquicas, sendo que esse percurso é uma busca em profundidade. Por meio de podas, um grande número de pares de objetos espaciais são descartados. Essa estratégia apresenta o melhor desempenho dentre as já existentes.

Na literatura são encontrados diversos trabalhos voltados à proposição de índices e algoritmos de junção espacial. A maioria desses algoritmos dedica-se à fase de filtragem, ou seja, à seleção de pares candidatos. Dentre esse grande número de trabalhos, destacam-se: (i) trabalhos que são baseados em índices da família R-Tree (Bae et al., 2006, 2010; Brinkhoff 
et al., 1993); (ii) trabalhos que fazem um levantamento sobre algoritmos, modelos de custo e técnicas de otimização (Jacox e Samet, 2007; Mamoulis, 2009; Mamoulis et al., 2005); (iii) trabalho que traz um estudo de planos de execução de consultas (Papadopoulos et al., 1999); (iv) trabalho que realiza um estudo sobre a aplicação de índices espaciais em junções espaciais (Günther, 1993); (v) trabalho que utiliza aproximações progressivas dos objetos espaciais (Brinkhoff et al., 1994); e (vi) trabalhos que são baseados em outros tipos de estruturas (Aref, 2007; Back et al., 2010; Chen e Chang, 2009; Jacox e Samet, 2003). Além desses trabalhos, Gatti e Magalhães (2000) descrevem um estudo de métodos de estimativas de custo e análise de fatores que afetam o desempenho de junções espaciais, enquanto que Rocha (2003) realiza um estudo de desempenho de junções espaciais comparando modelos analíticos e resultados experimentais.

\section{2 Índices para Data Warehouses Convencionais}

Índices convencionais, em geral, não são adequados para DWs devido ao grande volume de armazenamento do próprio índice e a tempos proibitivos de respostas às consultas. Nesta seção são descritos o índice de Projeção (seção 3.2.1) e o índice Bitmap de junção (seção 3.2.2), propostas voltadas à solução desse problema.

\subsection{1 Índice de Projeção}

Dada uma tabela $\mathcal{T}$ e um atributo $A$, o índice de Projeção (Projection Index) (O'Neil e Quass, 1997) de $A$ consiste no armazenamento sequencial dos valores de $A$ ordenados pelo Rowld, o qual é um número que identifica e ordena as tuplas de uma tabela. Se o tamanho do atributo $A$ é 4 Bytes, então é possível armazenar 1012 valores de $A$ em uma página de disco de 4 KBytes.

\begin{tabular}{|c|c|c|c|c|c|}
\hline Rowld & s_suppkey & s_address & s_city & s_nation & s_region \\
\hline 0 & 1 & A & Vietnam 2 & Vietnam & Asia \\
\hline 1 & 2 & B & France 5 & France & Europe \\
\hline 2 & 3 & C & Romania 2 & Romania & Europe \\
\hline 3 & 4 & D & Algeria 6 & Algeria & Africa \\
\hline 4 & 5 & $E$ & Algeria 0 & Algeria & Africa \\
\hline
\end{tabular}

(a) Fragmento da tabela supplier do SSB.

\begin{tabular}{|c|cc}
\multicolumn{1}{c|}{ s_nation } & página & slot \\
\cline { 1 - 1 } Vietnam & 0 & 0 \\
\cline { 1 - 1 } France & 0 & 1 \\
\cline { 1 - 1 } Romania & 1 & 0 \\
\cline { 1 - 1 } Algeria & 1 & 1 \\
\cline { 1 - 1 } Algeria & 2 & 0 \\
\hline
\end{tabular}

(b) Índice de Projeção para s_nation.

Figura 3.3: Índice de Projeção - adaptada de (Siqueira et al., 2009a).

Na Figura 3.3 é ilustrado um exemplo de índice de Projeção para o atributo s_nation do fragmento da tabela supplier do benchmark SSB. A partir do Rowld de uma tupla, a posição em que se encontra o valor de s_nation na sequência é diretamente calculada pelo Rowld e 
o valor pode então ser recuperado. O inverso também se aplica, ou seja, dado um valor do atributo s_nation em uma certa posição, o Rowld é facilmente calculado. No exemplo da Figura 3.3, os Rowlds são enumerados a partir de 0 e as páginas armazenam 2 valores. Dada uma tupla com Rowld igual a $n$, o valor correspondente do atributo $s_{-}$nation está localizado na página $p$ e slot $s$, onde $p=n \div 2$ e $s$ é o resto da divisão de $n$ por 2 . O Rowld da tupla correspondente é fornecido pela expressão Rowld $=p * 2+s$. Por exemplo, o valor de s_nation para o Rowld 3 está localizado na página 1 e slot 1 . De maneira inversa, o Rowld correspondente ao valor de s_nation localizado página 2 e slot 0 é 4 , ou seja, Rowld $=2 * 2+0$.

O índice de Projeção é análogo a um particionamento vertical dos dados. No entanto, considera-se que ele seja uma estrutura auxiliar para melhorar o desempenho de consultas e que os dados permanecem armazenados da forma convencional.

\subsection{2 Índice Bitmap de Junção}

Um índice Bitmap para um atributo $A$ é uma sequência ordenada de todos os valores distintos que esse atributo assume. Cada valor dessa sequência aponta para uma sequência de bits. $O$ número de elementos dessa sequência de bits é igual ao número de tuplas da tabela à qual esse atributo $A$ pertence. Portanto, cada bit aponta para uma tupla da tabela. Por exemplo, se o bit de um vetor Bitmap, correspondente a um valor $x$ do atributo $A$, possui valor igual a 1 , significa que a tupla correspondente possui o valor $x$ no atributo $A$. Caso o valor do bit seja 0 , a tupla não possui esse valor no atributo $A$.

Em geral, consultas que incidem sobre o esquema estrela de um DW exigem a realização de várias operações de junção das tabelas de dimensão com a tabela de fatos. Essa operação é conhecida como junção estrela e trata-se de uma operação muito custosa, pois uma tabela de fatos usualmente contém da ordem de milhões de tuplas. Para evitar o cálculo da junção estrela, um índice Bitmap pode ser construído para indexar os atributos das tabelas de dimensão na tabela de fatos, ou seja, pode-se criar um índice Bitmap para um dado atributo $C$ de uma tabela de dimensão com a finalidade de indicar o conjunto de tuplas na tabela de fatos a ser juntada com um dado valor de $C$. Esse tipo de construção é chamado de índice Bitmap de junção (O'Neil e Graefe, 1995).

Na Figura 3.4 é exibido um índice Bitmap de junção entre as tabelas supplier e lineorder, ambas do benchmark SSB. Na Figura 3.4.c são ilustrados dois índices Bitmap de junção: um definido sobre o atributo s_address e outro sobre o atributo s_nation. Note que o atributo s_suppkey da tabela de dimensão supplier é referenciado pelo atributo lo_suppkey da tabela de fatos lineorder. Suponha uma consulta que busque pelo valor s_address = 'a'. Nesse caso, é necessário apenas verificar os bits do vetor Bitmap correspondente ao valor 'a' que possuam valor igual a 1 e recuperar as respectivas tuplas da tabela lineorder. Dessa forma, o índice 


\begin{tabular}{|c|c|c|c|c|c|}
\hline Rowld & s_suppkey & s_address & s_city & s_nation & s_regior \\
\hline 0 & 1 & a & Vietnam 2 & Vietnam & Asia \\
\hline 1 & 2 & b & France 5 & France & Europe \\
\hline 2 & 3 & c & Romania 2 & Romania & Europe \\
\hline 3 & 4 & d & Algeria 6 & Algeria & Africa \\
\hline 4 & 5 & e & Algeria 0 & Algeria & Africa \\
\hline
\end{tabular}

(a) Fragmento da tabela de dimensão supplier do SSB.

\begin{tabular}{|c|c|c|}
\multicolumn{1}{r|}{ lo_suppkey } & lo_custkey & lo_revenue \\
\hline 1 & 235 & 20785 \\
\hline 1 & 512 & 166751 \\
\hline 2 & 512 & 252145 \\
\hline 3 & 235 & 197451 \\
\hline 3 & 512 & 152389 \\
\hline 3 & 106 & 217410 \\
\hline 4 & 235 & 200489 \\
\hline 5 & 106 & 182877 \\
\hline
\end{tabular}

(b) Fragmento da tabela de fatos lineorder.
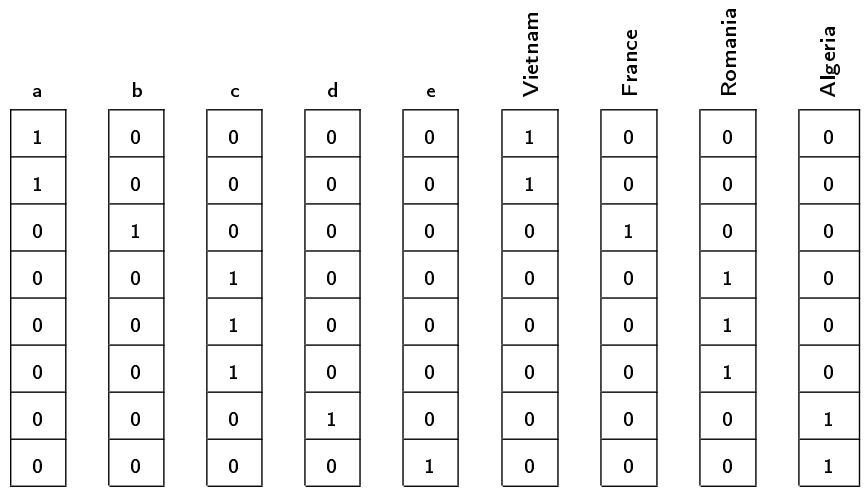

(c) Índice Bitmap de junção para s_address e s_nation.

Figura 3.4: Índice Bitmap de junção - adaptada de (Siqueira et al., 2009a).

Bitmap de junção evita a junção entre as tabelas supplier e lineorder e permite um rápido processamento da consulta por meio de operações lógicas bit a bit.

$O$ índice Bitmap de junção tem um desempenho satisfatório na resolução de consultas mesmo quando o número de dimensões indexadas é grande, sendo, portanto, amplamente utilizado na indexação de DWs. Entretanto, índices Bitmap podem sofrer problemas causados pela alta cardinalidade de atributos. Isso acarreta em alto custo de armazenamento e perda de desempenho devido aos vetores Bitmaps extremamente grandes e esparsos. Esses problemas podem ser atenuados por técnicas de otimização, a saber: binning (Rotem et al., 2005; Stockinger et al., 2004; Wu et al., 2008), compressão (Antoshenkov, 1995; Goyal et al., 2006; Wu et al., 2006) e codificação (Chan e loannidis, 1999; O'Neil e Quass, 1997; Wu e Buchmann, 1998).

Na seção 3.2.2.1 é descrito o software FastBit para a manipulação de índices Bitmap, o qual foi usado nas implementações realizadas durante o desenvolvimento desta dissertação.

\subsubsection{FastBit}

O FastBit (Wu, 2005) é um software livre que fornece uma série de ferramentas para a manipulação de índices Bitmap. É uma ferramenta usada em vários projetos de pesquisa, por exemplo no trabalho de Siqueira (2009). O software é escrito em $\mathrm{C}++$, mas outras linguagens de programação podem acessá-lo por meio de bibliotecas. Sua primeira versão comercial foi o Model 204 (O’Neil, 1987). Além disso, o FastBit utiliza por padrão a técnica de compressão 
Word Aligned Hybrid (WAH) (Wu et al., 2006) e oferece como opções as técnicas de binning e codificação.

A organização dos dados é feita em partições. O número de partições depende do tamanho da tabela, ou seja, do volume de dados. Cada partição é armazenada em um diretório e possui um arquivo de metadados, os quais descrevem os dados indexados na partição (e.g, número de colunas, número de linhas, técnica de otimização utilizada). Para cada coluna, são descritos o nome da coluna, tipo de dado, valores mínimo e máximo. Além do arquivo de metadados, cada coluna é representada por no mínimo dois arquivos: um com os possíveis valores da coluna em sequência ordenada e outro com o índice Bitmap correspondente. Essa ordenação funciona como ponteiros implícitos para os vetores. Dependendo do tipo e distribuição dos dados, arquivos adicionais podem ser criados na indexação de uma coluna.

\subsection{Considerações Finais}

Neste capítulo foram descritos dois dos mais referenciados índices espaciais existentes na literatura, a R-Tree e a $\mathrm{R}^{*}$-Tree. Também foram destacadas as principais estratégias utilizadas pelos algoritmos de junção espacial. Além disso, foram discutidos dois índices para DWs convencionais: índice de Projeção e índice Bitmap de junção. Por fim, foi feita uma breve descrição do software livre FastBit.

Por um lado, apesar dos índices R-Tree e $\mathrm{R}^{*}$-Tree serem bastante usados na indexação de objetos espaciais com suporte a ponto, linhas e polígonos, eles não são apropriados para DWGs por não evitarem a junção estrela. Ademais, os algoritmos de junção espacial abordados na seção 3.1.3 não são baseados em índices para DWGs, tornando seu uso inadequado nesses ambientes. Por outro lado, apesar do índice Bitmap de junção ser amplamente utilizado na indexação de DW convencionais, evitando a necessidade de junção estrela, esse índice não provê suporte à indexação de DWGs, isto é, não indexa dados espaciais.

No próximo capítulo são descritos trabalhos correlatos às estratégias propostas nesta dissertação, os quais consistem em índices para DWGs. 


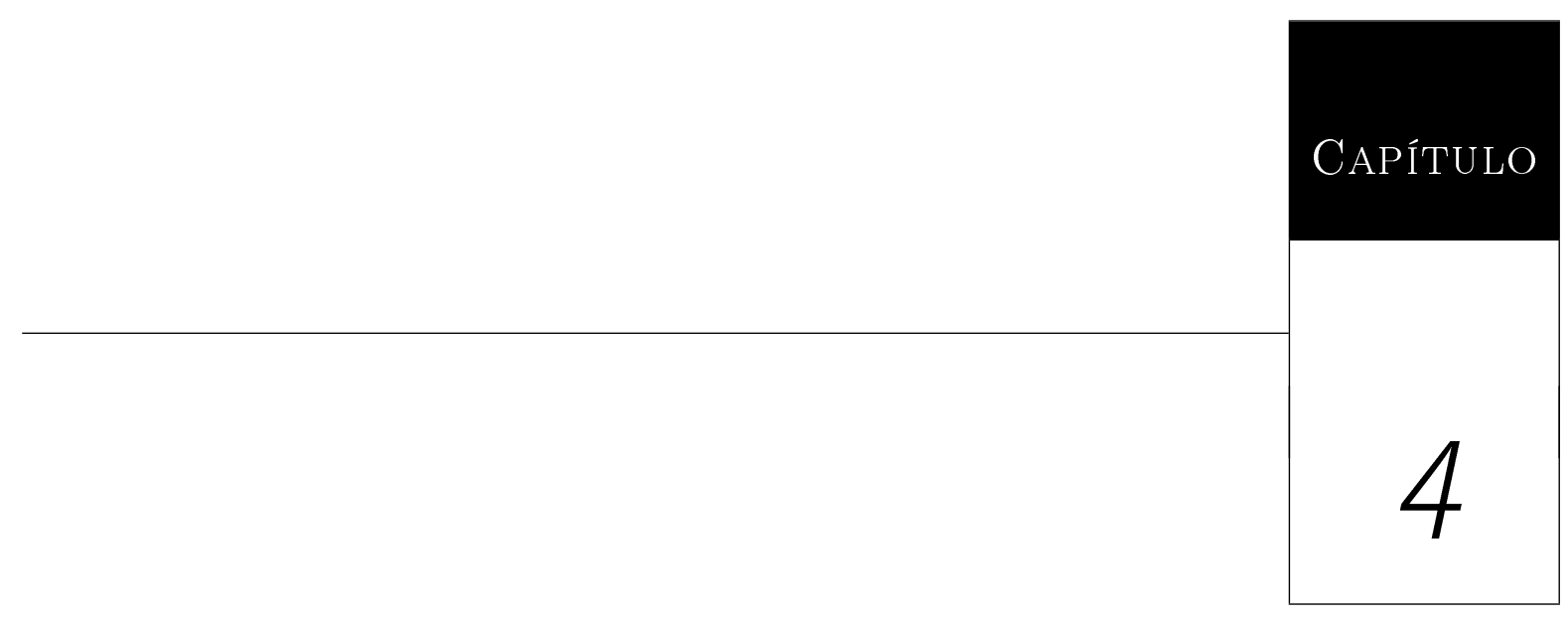

\section{Índices para Data Warehouses Geográficos}

A indexação de DWGs é um tema de pesquisa desafiador, sendo que poucos trabalhos na literatura vislumbram esse desafio. Trabalhos correlatos relacionados às estratégias de processamento propostas nesta dissertação são os índices aR-tree (Papadias et al., 2001) (seção 4.1), e suas extensões a3DR-tree e aRB-tree (Papadias et al., 2002) (seção 4.2). Outro trabalho correlato, o qual envolve junção espacial em DWGs, consiste na proposta dos índices SJELI e SJALI (Mohan et al., 2008) (seção 4.3). As propostas dos índices SB-index (Siqueira et al., 2009b, 2008, 2009a) e HSB-index (Siqueira et al., 2012) são descritas nas seções 4.4 e 4.5, respectivamente. Em especial, o SB-index é um índice precursor ao trabalho desenvolvido nesta dissertação, ou seja, ele é usado como base para a proposta das estratégias de processamento de consultas SOLAP drill-across e com junção espacial. O capítulo é finalizado na seção 4.6, com as considerações finais.

\section{1 aR-tree}

A aggregate R-tree (aR-tree) (Papadias et al., 2001), é um índice para DWGs com enfoque em hierarquias espaciais ad hoc. Desde que métodos conhecidos de pré-agregação para o processamento de operações OLAP não são aplicáveis a esse tipo de hierarquia, a aR-tree foi 
proposta com o intuito de desenvolver um índice que integre a pré-agregação de resultados e a indexação espacial.

Dado um conjunto de objetos (e.g., carros, usuários de celular) que possuem localização espacial, ou seja, coordenadas $x$ e $y$, a aR-tree é um índice voltado para responder questões nas quais há interesse na quantidade desses objetos em certas regiões do espaço. Um exemplo de consulta desse tipo, pertinente em sistemas que envolvam o controle de tráfego é: "Qual a quantidade de carros localizados em estradas que intersectam uma dada janela de consulta?".

A estrutura de dados da aR-tree consiste em uma R-tree que armazena para cada MBR o valor da função de agregação dos objetos nele contidos. O método de particionamento do espaço é o mesmo utilizado na R-tree e o índice é construído no grau mais fino de granularidade dos objetos espaciais. O exemplo da Figura 4.1 refere-se a um sistema de monitoramento do tráfego de carros nas ruas de uma cidade. Carros são representados por pontos e ruas por linhas. A entrada $\left(r_{2}, 3\right)$ na aR-tree indica que existem três carros na rua cujo MBR é $r_{2}$. O mesmo se aplica para as outras entradas. Os resultados agregados de um nível inferior são agregados no próximo nível superior. Por exemplo, a entrada $\left(R_{1}, 6\right)$ armazena os resultados agregados das entradas $\left(r_{2}, 3\right),\left(r_{3}, 1\right)$ e $\left(r_{5}, 2\right)$. Nesse exemplo, a função de agregação utilizada é soma, mas outras funções podem ser utilizadas. Uma mesma entrada pode guardar mais de um valor agregado, correspondentes a funções ou objetos diferentes.

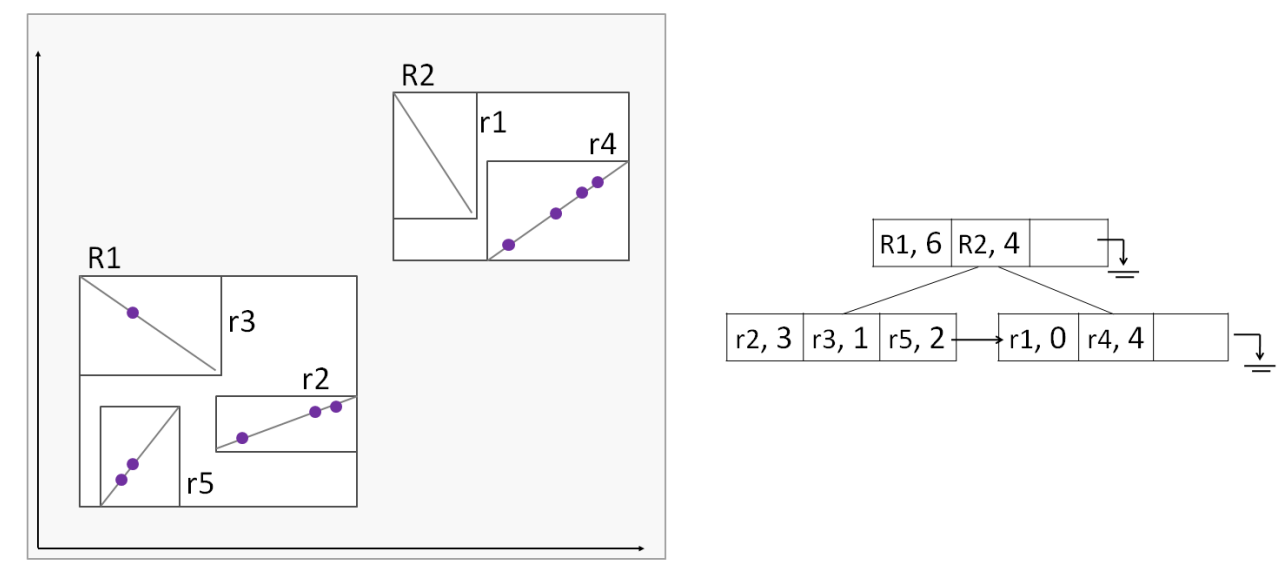

Figura 4.1: aR-tree - adaptada de (Papadias et al., 2001)

Segundo Papadias et al. (2001), a aR-tree define uma hierarquia sobre os MBRs e forma um grafo de derivação. Seja $E_{l}$ o conjunto de resultados materializados de todos os nós de uma aR-tree do nível $l$. $E_{l}$ pode ser obtido por meio dos resultados agregados armazenados em cada nó do nível $l-1$, ou seja, a partir de $E_{l-1}$. Portanto $E_{l+1} \preceq E_{l} \forall l \in[0, h]$, onde $h$ é a altura da árvore. Na Figura 4.2 é apresentado o grafo de derivação do exemplo da Figura 4.1.

Caso os carros do exemplo fossem classificados em tipos, e as consultas buscassem por valores agregados de tipos específicos, a estrutura da aR-tree precisaria guardar um valor agregado para cada tipo de carro. Isso poderia ser feito por meio de um arranjo multidimensional 


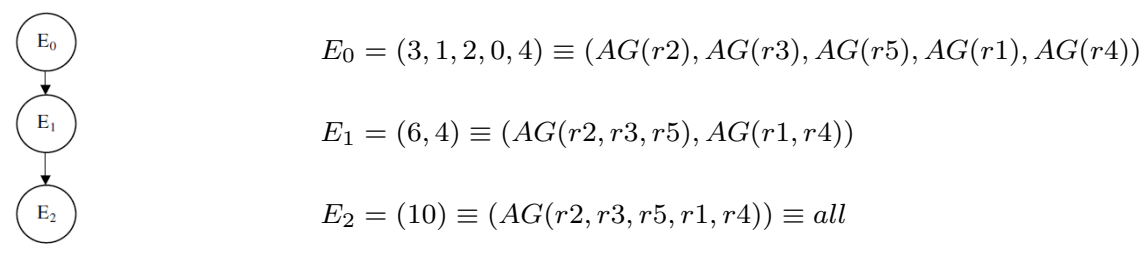

Figura 4.2: Grafo de derivação da aR-tree - extraída de (Papadias et al., 2001).

(vetor) apontado por cada entrada da aR-tree. Nesse vetor seria armazenado o valor agregado para cada tipo de carro. É possível perceber que a existência de um número grande de tipos de carros, ou até outras dimensões, geraria arranjos grandes e um alto custo associado para acessá-los.

\subsubsection{Processamento de Consultas}

Um exemplo do tipo de consulta considerada nessa seção é: "Qual a quantidade de carros localizados em estradas que intersectam uma dada janela de consulta?". O algoritmo de busca é similar ao aplicado em uma consulta que utiliza a R-tree. O processamento da consulta inicia-se pela raiz e percorre a árvore até as folhas. Dado o MBR de uma entrada e uma janela de consulta $(J C)$, três situações podem ocorrer:

- O MBR está contido na JC, portanto o valor da função de agregação armazenado na entrada é recuperado.

- O MBR e a JC são disjuntos, portanto o valor da função de agregação é descartado.

- O MBR sobrepõe parcialmente a JC, portanto é necessário visitar todos seus nós filhos e executar o mesmo processo.

Estimativas podem ser usadas caso entradas de nós folhas intersectem parcialmente a JC. Há diferenças sutis entre a R-tree e a aR-tree em consultas que utilizam janela de consulta. Na R-tree, se a janela de consulta for muito grande, a utilização do índice se torna dispensável e é melhor optar por uma busca sequencial. Já para a aR-tree, tem-se as seguintes situações:

- Se a JC é muito grande, muitos nós de níveis intermediários da aR-tree estão contidos na janela e os resultados armazenados são utilizados, evitando a visita a muitos nós.

- Se a JC for muito pequena, a aR-tree é usada como um índice espacial, selecionando apenas porções de interesse do conjunto de dados.

\section{2 a3DR-tree e aRB-tree}

Em (Papadias et al., 2002), o foco de interesse deixa de ser a busca por informações sobre localização de objetos individuais em um determinado instante de tempo em uma certa região 
do espaço e passa a ser a busca de informações espaço-temporal sumarizadas, ou seja, informações baseadas no histórico da localização desses objetos. Dois cenários são considerados: indexação de dimensões espaciais estáticas e indexação de dimensões espaciais dinâmicas.

No primeiro cenário, dimensões espaciais estáticas, os objetos espaciais associados às regiões são fixos e apenas o valor agregado associado ao número de objetos em cada região é variável. Por exemplo, considere um conjunto fixo de ruas com carros em movimento. As ruas correspondem às regiões indexadas pela aR-tree e são fixas, enquanto os carros correspondem aos objetos associados a essas regiões e seu número é variável, pois os carros estão em movimento. Já no segundo cenário, dimensões espaciais dinâmicas, esse conjunto é variável, ou seja, objetos espaciais associados às regiões podem mudar de tamanho, serem excluídos e novos objetos espaciais podem aparecer. O contexto desta dissertação de mestrado diz respeito a objetos espaciais que não alteram sua geometria ao longo do tempo (e.g., cidades e países), logo, somente o primeiro cenário é considerado nessa seção.

$\mathrm{Na}$ indexação de dimensões espaciais estáticas, o objetivo é apenas manter dados históricas relacionadas às mudanças dos valores das medidas agregadas e, para isso, é apresentada uma primeira abordagem, a aggregate 3-dimensional R-tree (a3DR-tree). Cada entrada da a3DRtree possui quatro campos: o MBR do objeto espacial, um ponteiro para o nível inferior ou para a própria tupla, o intervalo de tempo no qual o valor da medida agregada é válido e o valor da medida agregada. A cada mudança no valor de uma medida, uma nova entrada é inserida na árvore.

A Figura 4.3.c ilustra uma a3DR-tree construída a partir do cubo de dados ilustrado na Figura 4.3.b e da R-tree representada na Figura 4.3.a. A cada mudança no valor de uma medida agregada, uma nova entrada é gerada. Por exemplo, no instante de tempo T2, os valores das medidas das regiões $R_{2}$ e $R_{3}$ mudam de 75 para 80 e de 132 para 127, respectivamente. Duas novas entradas são geradas, $\left(R_{2},[\mathrm{~T} 2, \mathrm{~T} 2], 80\right)$ e $\left(R_{3},[\mathrm{~T} 2, \mathrm{~T} 2], 127\right)$. Essas mudanças são propagadas para o nível superior, e o valor de $R_{5}$ passa de 225 para 230 e de $R_{6}$ de 144 para 139.

A a3DR-tree possibilita uma integração entre as dimensões espacial e temporal em uma única estrutura. Sua grande desvantagem é a redundância gerada pelo fato de um mesmo MBR poder ser armazenado várias vezes, gerando um desperdício de espaço de armazenamento. Outro fator negativo é o pequeno fan-out gerado, ou seja, pequeno número de conexões entre uma grande quantidade de entradas. Várias versões de uma mesma entrada (e.g., entrada $R_{5}$ ) estão ligadas ao mesmo nó no nível inferior (e.g., nó que possui as entradas $R_{1}$ e $R_{2}$ ). Isso gera uma perda de desempenho no processamento de consultas.

A segunda abordagem, a aggregate RB-tree (aRB-tree), armazena as regiões em uma única R-tree. Cada entrada da R-tree mantém um valor que corresponde à soma do número de objetos presentes nessa entrada ao longo do tempo. Além disso, cada entrada da R-tree também mantém um ponteiro para uma B-tree que armazena o histórico desses valores para aquela entrada. 


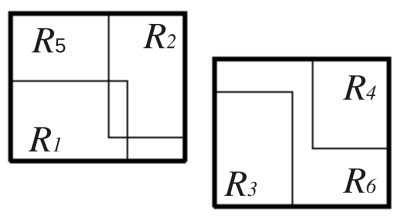

(a) MBRs da R-tree - adaptada de (Papadias et al., 2002).

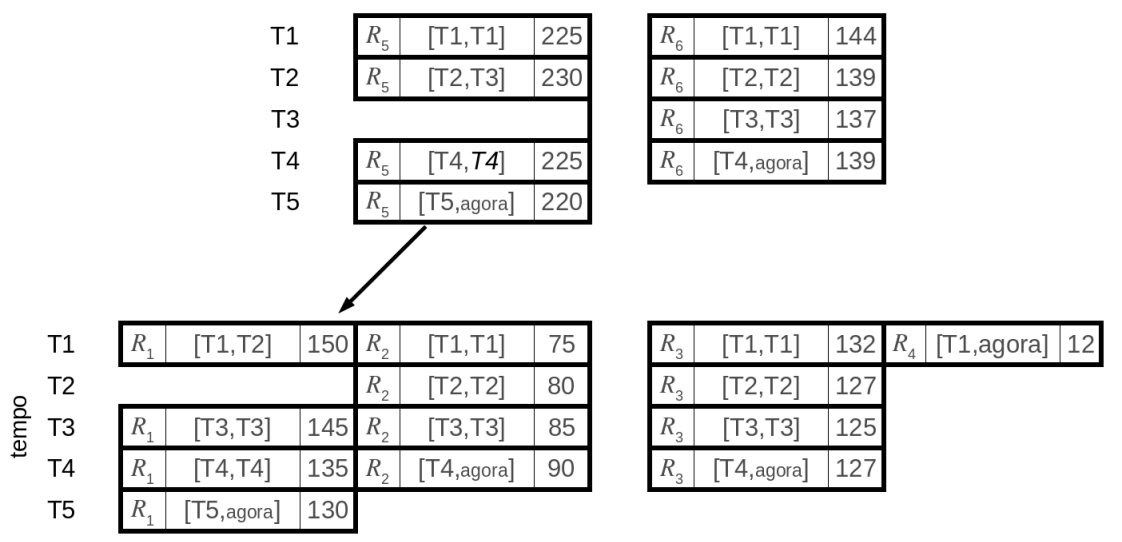

(c) a3DR-tree.

Figura 4.3: a3DR-tree

No exemplo da Figura 4.4, a entrada $R_{5}$ da R-tree registra um total de 1130 objetos ao longo do tempo. As entradas dos nós folhas de sua B-tree correspondente indicam que a região $R_{5}$ possui 225 objetos no instante de tempo 1, 230 no instante 2, 225 no instante 4 e 220 no instante 5. Note que não há entrada referente ao instante 3, ou seja, o número de objetos não se alterou e nesse instante há 230 objetos, indicado pela entrada $(2,230)$. As entradas de nós superiores armazenam valores agregados por um determinado período de tempo. No exemplo, a entrada $(1,685)$ da B-tree de $R_{5}$ armazena um total de objetos igual a 685 , correspondente ao intervalo $[1,3]$.

Seja uma consulta que utilize a JC ilustrada na Figura 4.5 e que requeira o total de objetos contidos em regiões que intersectam a JC no intervalo [1,3]. A região $R_{5}$ está contida na JC, então o valor 685, correspondente ao intervalo desejado, é recuperado na raiz da B-tree de $R_{5}$. A região $R_{6}$ intersecta parcialmente a janela de busca, portanto é preciso descer um nível na $\mathrm{R}$-tree e checar as regiões $R_{3}$ e $R_{4}$. Somente a região $R_{3}$ intersecta a JC e portanto sua Btree correspondente deve ser percorrida. No primeiro nível da B-tree de $R_{3}$, a entrada $(1,259)$ representa 259 objetos no intervalo [1,2]. Portanto, o valor 259 é recuperado e parte-se para 


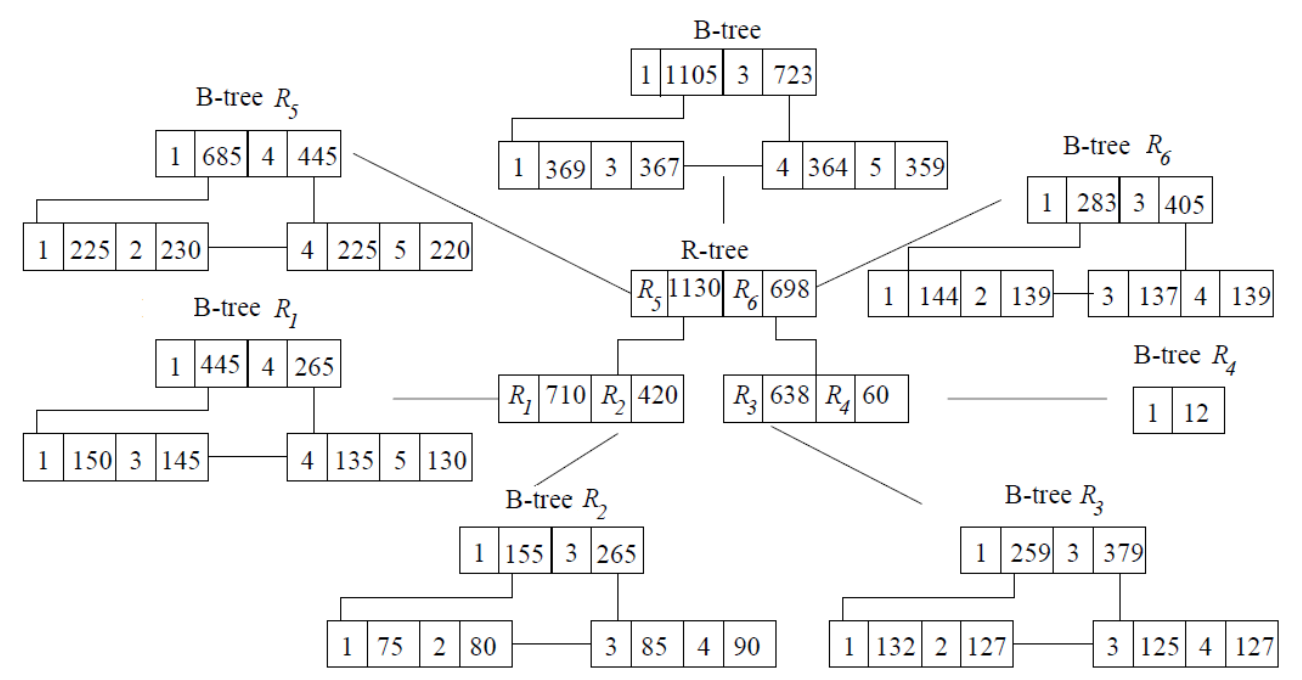

Figura 4.4: aRB-tree - adaptada de (Papadias et al., 2002).

a busca do valor no intervalo [3,3], o qual é encontrado na entrada $(3,125)$ do nível inferior. A resposta da consulta é a soma dos valores $685+259+125$, ou seja, 1069 objetos.

Quando a frequência segundo a qual os valores agregados mudam é muito alta, a estrutura do índice pode se tornar extremamente grande, gerando assim um custo alto de acesso às várias estruturas.

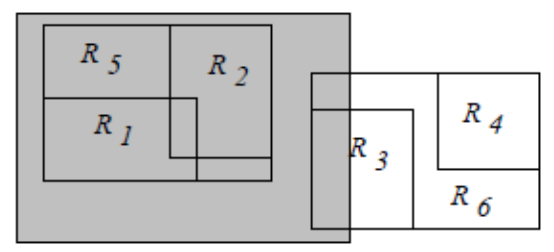

Figura 4.5: Exemplo de janela de consulta - extraída de (Papadias et al., 2002).

\subsection{SJELI e SJALI}

Em seu trabalho, Mohan et al. (2008) propõem duas variações do Self-Join Index capazes de responder a um conjunto de consultas espaciais estatísticas de forma eficiente: os índices SJELI e SJALI. O estudo de caso utilizado como base no trabalho é um pacote popular de programas chamado CrimeStat, o qual realiza estatísticas baseadas na distribuição espacial dos dados. Os dados são mantidos em um DWG que não sofre alterações frequentes e dizem respeito à incidência e localização de crimes, sendo a localização representada por meio de pontos. Um exemplo de análise estatística que pode ser realizada é verificar a existência de regiões concentradas de crimes. Esse tipo de análise faz um mapeamento da criminalidade e pode auxiliar o sistema de segurança pública. 
As consultas espaciais estatísticas são classificadas como $W$-Queries pelo fato de serem frequentemente realizadas sobre um tipo de representação matricial chamada $W$ Matrix. $W$ Queries envolvem cálculos repetidos de relações de vizinhança entre os vértices (e.g., vértices vizinhos de um vértice, vértices sucessores de um vértice, vértices antecessores de um vértice). Um exemplo de $W$-Query é: "Os dados são clusterizados?". Esse exemplo está diretamente relacionado com o cálculo de uma medida estatística chamada Ripley's K function, a qual envolve o cálculo, para todos os vértices, de quantos vértices estão localizados dentro de um certo raio.

O Self-Join Index é representado por um grafo não direcionado que consiste em um conjunto de vértices e arestas. Os vértices representam os pontos, que no exemplo corrente indica a localização de um crime, enquanto que as arestas representam relações de vizinhança entre dois vértices. Uma aresta pode ter um peso associado a uma interação espacial, distância ou adjacência. O grafo pode ser representado por uma lista de adjacência (adjacency list) ou por uma lista de arestas (edge list), como ilustrado na Figura 4.6, onde $\mathrm{R} i$ e $\mathrm{N} i$ representam as relações de vizinhança e os nós, respectivamente.

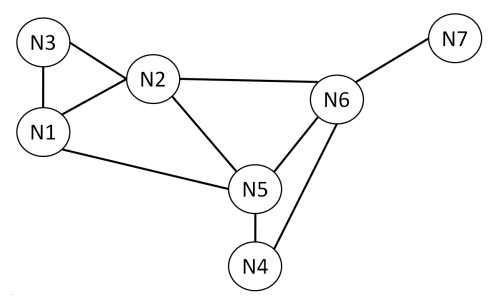

(a) Grafo de vizinhança de R1.

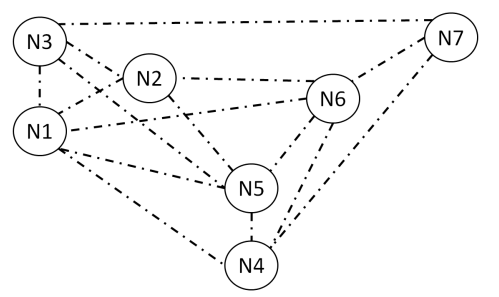

(b) Grafo de vizinhança de R2.

\begin{tabular}{|c|c|c|}
\hline $\mathrm{Ni}$ & Nu & $R_{k}\left(N_{i}, N_{J}\right)$ \\
\hline N1 & $\mathrm{N} 2$ & $\mathrm{R} 1(\mathrm{~N} 1, \mathrm{~N} 2)$ \\
\hline N1 & N3 & $\mathrm{R} 1(\mathrm{~N} 1, \mathrm{~N} 3)$ \\
\hline N1 & N5 & $\mathrm{R} 1(\mathrm{~N} 1, \mathrm{~N} 5)$ \\
\hline N1 & N4 & $\mathrm{R} 2(\mathrm{~N} 1, \mathrm{~N} 4)$ \\
\hline N1 & N6 & $\mathrm{R} 2(\mathrm{~N} 1, \mathrm{~N} 6)$ \\
\hline N2 & N1 & $\mathrm{R} 1(\mathrm{~N} 2, \mathrm{~N} 1)$ \\
\hline N2 & N3 & $\mathrm{R} 1(\mathrm{~N} 2, \mathrm{~N} 3)$ \\
\hline N2 & N5 & $\mathrm{R} 1(\mathrm{~N} 2, \mathrm{~N} 5)$ \\
\hline N2 & N6 & $\mathrm{R} 1(\mathrm{~N} 2, \mathrm{~N} 6)$ \\
\hline$\ldots$ & $\ldots$ & $\ldots$ \\
\hline
\end{tabular}

(c) SJELI de R1 e R2.

\begin{tabular}{|c|c|}
\hline Ni & Lista de Adjacência \\
\hline N1 & {$[\mathrm{N} 2: R 1, N 3: R 1, N 5: R 1, N 4: R 2, N 6: R 2]$} \\
\hline N2 & {$[\mathrm{N} 1: R 1, N 3: R 1, N 5: R 1, N 6: R 1]$} \\
\hline$\ldots$ & $\ldots$ \\
\hline
\end{tabular}

(d) SJALI de R1 e R2.

Figura 4.6: Índices SJALI e SJELI - adaptada de (Mohan et al., 2008).

A variação que utiliza lista de arestas e é denominada Self-Join Edge List Index (SJELI). Nessa variação, o índice é organizado em uma lista com três colunas (Figura 4.6.c), sendo que as duas primeiras colunas indicam os vértices aos quais a aresta está conectada, e a terceira coluna contém o valor associado à relação $R$. Segundo Mohan et al. (2008), essa representação não fornece informações sobre sucessores e antecessores. Além disso, é um desafio determinar um particionamento da estrutura de dados que otimize os custos de I/O. 
A segunda variação é o Self-Join Adjacency List Index (SJALI), o qual é baseado em lista de adjacência (Figura 4.6.d). Para cada vértice é criada uma lista que indica a quais vértices o primeiro está relacionado e por qual relação. Segundo Mohan et al. (2008), essa representação apresenta vantagens com relação à primeira, pois ela mantém uma lista de sucessores e antecessores que é um fator crítico no processamento de $W$-Queries. Além disso, o esquema de coloração usado no conjunto de operações realizado pelas $W$-Queries podem facilmente explorar essa representação no fornecimento de sucessores e antecessores. Em grafos coloridos, vértices sucessores possuem a mesma cor e antecessores possuem cores diferentes.

\subsection{SB-index}

O Spatial Bitmap Index (SB-index) (Siqueira et al., 2009a) é um índice para DWG com enfoque em hierarquias espaciais predefinidas. Sua proposta é introduzir o índice Bitmap de junção na indexação de tabelas de dimensão com atributos espaciais, proporcionando suporte à multidimensionalidade de maneira eficiente.

Dada uma consulta que utiliza predicado espacial, o SB-index filtra os objetos espaciais e evita a computação da junção estrela por meio da transformação do predicado espacial em um predicado convencional, o qual é combinado com os demais predicados convencionais da consulta. Isso possibilita a resolução da consulta contendo somente predicados convencionais por meio do uso de índices Bitmap de junção. O SB-index provê suporte às consultas analíticas dos tipos roll-up e drill-down.

Na seção 4.4 .1 são detalhadas a estrutura de dados e a construção do SB-index. O processamento de consultas é descrito na seção 4.4.2.

\subsubsection{Estrutura de Dados e Construção do Índice}

A estrutura de dados do SB-index mantém, para cada entrada da tabela de dimensão espacial, o valor da chave primária e o MBR do objeto espacial. Conceitualmente, o SB-index é um vetor unidimensional de $n$ entradas do tipo sbitvector, onde $n$ é o número de objetos espaciais da tabela de dimensão espacial indexada. As entradas e seus respectivos vetores de bits são mantidos em ordem crescente dos valores de chave e, consequentemente, a i-ésima entrada do SB-index aponta para o i-ésimo vetor de bits.

Sbitvector é um tipo de dados definido por dois campos, key e mbr. O campo key é do tipo inteiro e armazena um valor chave que referencia a chave primária do objeto espacial. O campo $m b r$ é um vetor de quatro elementos do tipo double que armazena as quatro coordenadas que definem o MBR do objeto espacial: $x_{\min }, y_{\min }, x_{\max }$ e $y_{\max }$. Cada objeto do tipo sbitvector possui um tamanho $s$, onde $s=\operatorname{sizeof}($ int $)+4 *$ sizeof(double). A estrutura de dados do SB-index é ilustrada na Figura 4.7. 
O vetor SB-index é mantido como um arquivo sequencial em disco, com páginas de tamanho fixo. Considerando que um dado do tipo inteiro tem 4 Bytes e do tipo double tem 8 Bytes, cada entrada do SB-index possui um tamanho $s=4+4 * 8$, ou seja, 36 Bytes. Portanto, se uma página possui um tamanho $m$, cada página pode armazenar $\lfloor m / s\rfloor$ entradas. Considerando uma página de 4096 Bytes, seriam armazenadas $\lfloor 4096 / 36\rfloor=113$ entradas por página.

A construção do índice requer uma conexão com o DWG e é feita por meio de uma consulta SQL sobre a tabela de dimensão espacial. Essa consulta seleciona a chave primária e o MBR de seu respectivo atributo espacial e as classifica em ordem crescente de chave primária. Um buffer do tipo sbitvector é ultilizado para armazenar os dados que estão sendo extraídos pela consulta. Quando sua capacidade é esgotada, ele é escrito em uma página de disco. Esse processo é repetido até que todas as tuplas da tabela de dimensão espacial sejam processadas pela consulta. A construção do SB-index é ilustrada na Figura 4.8.

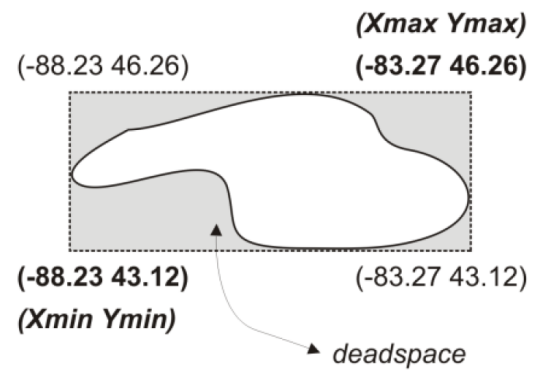

(a) Um objeto espacial e seu MBR.

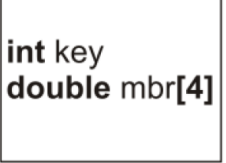

(b) O tipo sbitvector.

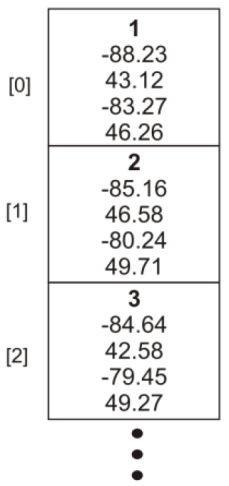

(c) O vetor SB-index.

Figura 4.7: Estrutura do SB-index - extraída de (Siqueira, 2009).

\subsubsection{Processamento de Consultas}

A primeira fase do processamento de uma consulta utilizando o SB-index é a fase de filtragem dos objetos espaciais. O SB-index é percorrido sequencialmente, e as chaves primárias dos objetos espaciais cujo MBR atende ao predicado espacial são selecionadas. As chaves primárias selecionadas formam uma coleção de chaves candidatas. De acordo com a Figura 4.9, a fase de filtragem corresponde aos números 1,2 e 3 .

Os objetos espaciais cujas chaves estão na coleção de candidatas são submetidos a uma próxima fase, chamada de refinamento (Figura 4.9, número 4), na qual os objetos espaciais são analisados considerando suas geometrias exatas. Na sequência, as chaves primárias dos objetos que satisfazem o predicado espacial são concatenadas a uma string, a qual substitui o predicado espacial, fazendo com que todos os predicados sejam convencionais. Por exemplo, na Figura 4.9, número 5, a consulta original é transformada substituindo-se seu predicado 


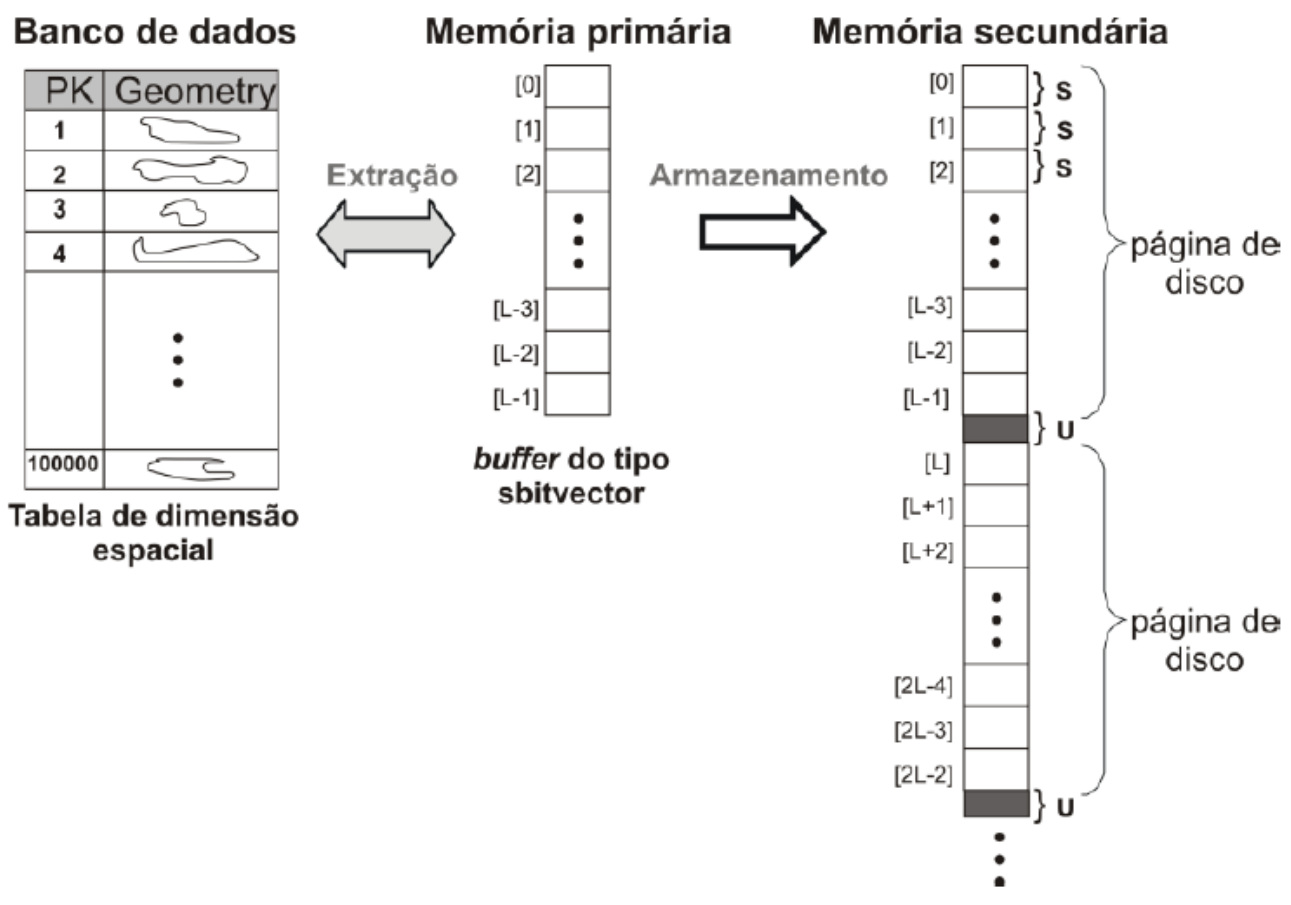

Figura 4.8: Construção do SB-index - extraída de (Siqueira, 2009).

espacial pelo predicado convencional $(P K=1 O R P K=9)$. Por fim, a consulta é resolvida pelo índice Bitmap de junção.

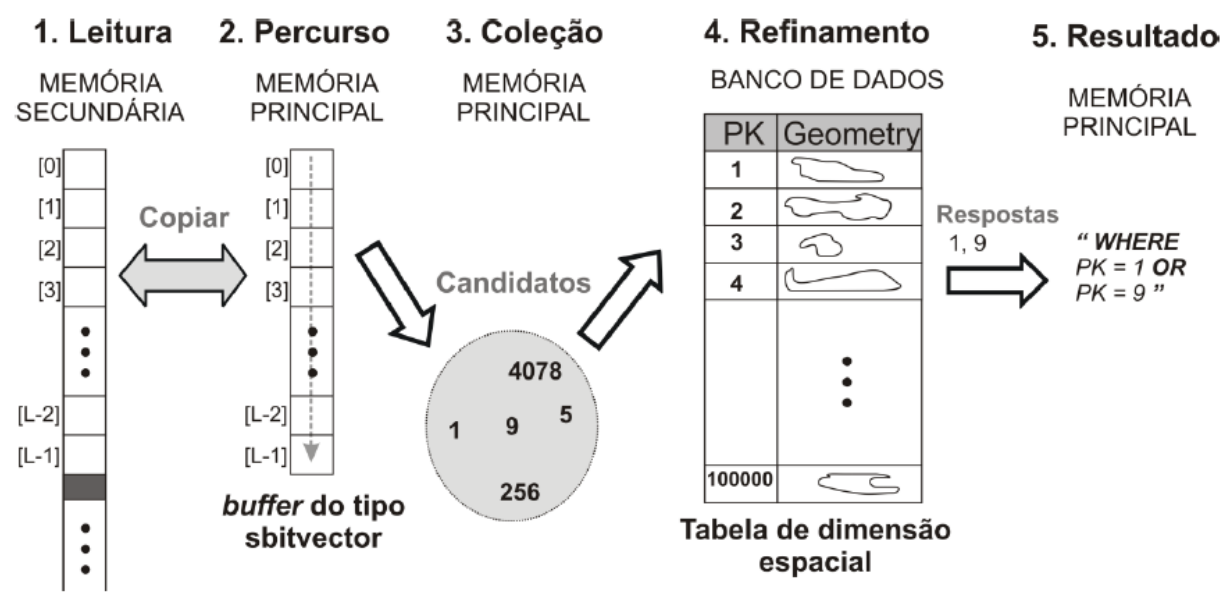

Figura 4.9: Processamento de consultas pelo SB-index - extraída de (Siqueira, 2009).

\subsection{HSB-index}

O Hierarchical Spatial Bitmap Index (HSB-index) (Siqueira et al., 2012) é uma variação do SB-index. Nessa nova proposta, a estrutura sequencial do índice espacial foi substituída por uma estrutura na forma hierárquica (como exemplo, a $\mathrm{R}^{*}$-Tree). Além disso, um bufferpool especializado foi adicionado para diminuir o número de acessos a disco. Esse buffer- 
pool é considerado especializado porque armazena, em memória principal, apenas páginas de disco do HSB-index. Assim, sempre que uma consulta é submetida para execução, o bufferpool é analisado com o objetivo de verificar se o nó desejado do índice espacial encontra-se armazenado em memória primária. Caso essa condição seja satisfeita, os dados armazenados no buffer-pool são utilizados e, portanto, não existe a necessidade de acessos a disco.

A estrutura de dados do HSB-index é ilustrada na Figura 4.10. Na estrutura hierárquica do índice espacial, cada nó folha aponta para um vetor Bitmap, o qual indica as ocorrências desse objeto espacial nas tuplas da tabela de fatos. Por exemplo, a entrada $<1, \mathrm{R} 1>$ do índice espacial representa o objeto espacial 1 cujo MBR é R1. De acordo com o vetor Bitmap apontado por essa entrada, o objeto espacial 1 ocorre na primeira tupla da tabela de fatos que este índice Bitmap de junção indexa.

Com relação à construção e à consulta usando o HSB-index, pode-se destacar que o algoritmo de construção da estrutura hierárquica é mesmo da $\mathrm{R}^{*}$-Tree, considerando $m=$ $0,4 M$. Além disso, o algoritmo de processamento de consultas é bem semelhante ao original, com diferença apenas na fase de filtragem, a qual incorpora as características e funcionalidades da $\mathrm{R}^{*}$-Tree.

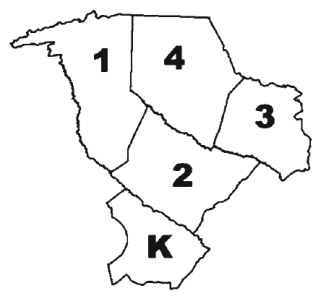

(a) Objetos espaciais.

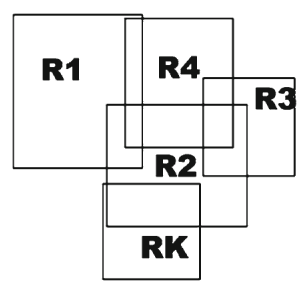

(b) MBRs.

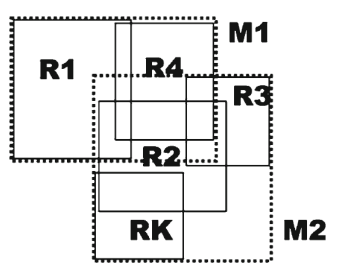

(c) Índice espacial.

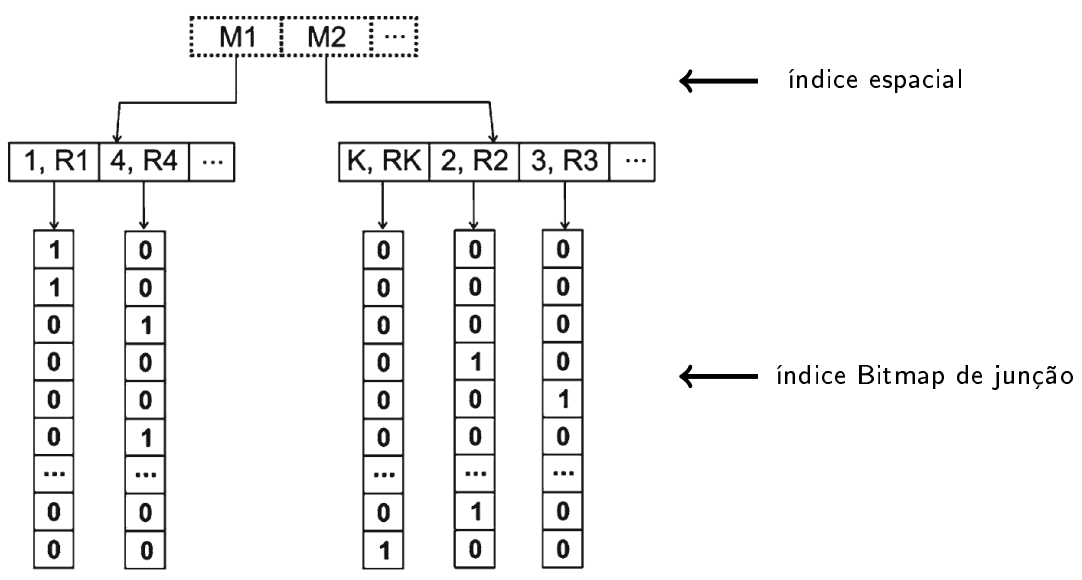

(d) HSB-index dos objetos espaciais.

Figura 4.10: Estrutura do HSB-index - extraída de (Siqueira et al., 2012). 


\subsection{Considerações Finais}

Este capítulo descreveu trabalhos que são correlatos às estratégias propostas nesta dissertação. O primeiro desses trabalhos, a aR-tree, é um índice multidimensional para DWGs com enfoque em hierarquias espaciais ad hoc. Já suas extensões, aRB-tree e a3DR-tree, são voltadas à indexação de DWs espaço-temporais. As variações do Self Join Index, SJALI e SJELI, são voltadas para operações de junção espacial necessárias em consultas espaciais estatísticas que realizam repetidos cálculos de relações de vizinhança entre pontos. Por fim, o SB-index e o HSB-index são índices para DWGs baseados no Índice Bitmap de junção. Enquanto que - SB-index tem uma estrutura sequencial, o HSB-index reusa técnicas de agrupamento de índices espaciais baseados em árvore, como a $\mathrm{R}^{*}$-Tree, para agrupar os objetos espaciais.

Apesar de já existirem trabalhos correlatos na literatura, esses trabalhos possuem limitações, as quais motivam o desenvolvimento das estratégias de processamento de consultas SOLAP drill-across e consultas SOLAP com junção espacial propostas nesta dissertação. Essas limitações são discutidas a seguir. Além do custo adicional requerido para acessar os arranjos multidimensionais, o índice aR-tree e suas extensões, a3DR-tree e aRB-tree, possuem outra restrição: nenhuma dessas estruturas vislumbra consultas SOLAP drill-across e consultas SOLAP com junção espacial. Os índices SJALI e SJELI, por sua vez, oferecem suporte para operações de junção espacial, porém eles vislumbram apenas autojunção de pontos e não evitam o cálculo da junção estrela. Adicionalmente, esses índices armazenam os resultados de junção espacial entre todos os pares de conjuntos espaciais e todos os possíveis relacionamentos topológicos, o que requer um grande espaço de armazenamento e a necessidade de se ter um conhecimento prévio de todos os predicados com os respectivos pares de conjuntos envolvidos, o que nem sempre é possível, principalmente em ambientes de consultas com predicados de junção espacial ad-hoc. Outra limitação refere-se ao fato de que os índices SJALI e SJELI não vislumbram consultas SOLAP drill-across. Por fim, o SB-index e o HSB-index enfocam apenas em consultas SOLAP drill-down e roll-up, não oferecendo suporte para consultas SOLAP drill-across e com junção espacial.

Segundo o nosso conhecimento, o SB-index e o HSB-index são os índices para DWGs mais rápidos existentes na literatura até o presente momento. Comparando esses dois índices, em seu trabalho, Siqueira et al. (2012) demonstraram que a escolha entre o uso do SB-index e do HSB-index depende da seletividade dos predicados espaciais envolvidos na consulta. Por um lado, o HSB-index deve ser usado quando as consultas requerem que somente poucos objetos espaciais sejam recuperados, desde que sua organização hierárquica garante que apenas um subconjunto dos objetos espaciais é analisado no processamento da consulta (i.e., consultas com baixa seletividade). Por outro lado, o SB-index deve ser usado principalmente quando as consultas requerem um número maior de objetos a serem processados. Nessa situação, ou seja, quando vislumbra-se uma gama variada de seletividades, a varredura sequencial provida pelo SB-index representa a técnica mais apropriada para o processamento de consultas SOLAP. 
Nos Capítulos 5 e 6 são apresentadas as propostas desenvolvidas nesta dissertação para o processamento de consultas SOLAP drill-across e consultas SOLAP com junção espacial, respectivamente. Essas propostas são baseadas no uso do SB-index, o qual além de ser muito eficiente, garante melhor indexação de consultas segundo uma gama variada de seletividades dos predicados espaciais. 


$\mathbf{a}$

\section{Processamento de Consultas SOLAP Drill-across}

Este capítulo tem como objetivo propor duas estratégias para o processamento de consultas SOLAP drill-across em DWGs, as quais são baseadas no índice espacial para DWGs SB-index. As estratégias de processamento que foram desenvolvidas consideram as seguintes características do SB-index: (i) enfoque em hierarquia espacial predefinida; (ii) reuso das características e funcionalidades do índice Bitmap de junção; e (iii) computação do predicado espacial primeiro, posterior transformação da resposta do predicado espacial em um predicado convencional, e processamento do predicado convencional gerado usando-se um índice Bitmap de junção. Assim, essas estratégias podem ser caracterizadas como uma extensão dos algoritmos do SBindex para enfocar consultas SOLAP drill-across. Em especial, a principal motivação para a proposta dessas estratégias refere-se ao fato de que nenhum trabalho na literatura é voltado ao processamento dessas consultas SOLAP complexas, como descrito no Capítulo 4.

Este capítulo está organizado da seguinte forma. Na seção 5.1 são detalhadas as propostas realizadas para o processamento de consultas SOLAP drill-across. Na seção 5.2 são discutidos os resultados dos testes de desempenho. O capítulo é finalizado na seção 5.3 , com as considerações finais. 


\subsection{Proposta}

Visando oferecer fundamentação para a proposta das estratégias para o processamento de consultas SOLAP drill-across, foram pesquisados dois aspectos desafiadores relacionados, os quais referem-se ao esquema de DWG e à definição de classes de consultas. Portanto, esta dissertação inclui as seguintes contribuições:

- A especificação de um conjunto de diretrizes que deve ser seguido para o projeto de um esquema de DWG que possibilite a execução de consultas SOLAP drill-across.

- A especificação de classes de consultas SOLAP drill-across que introduzem diferentes custos de processamento, de forma que essas consultas sejam executadas sobre um DWG projetado de acordo com as diretrizes propostas.

- A proposta de uma primeira estratégia, chamada de Divide, a qual consiste em uma abordagem baseada no índice SB-index para o processamento de consultas SOLAP drillacross.

- A proposta de uma segunda abordagem, chamada de Única, a qual também consiste em uma abordagem baseada no índice SB-index para o processamento de consultas SOLAP drill-across.

Essas contribuições são detalhadas nas seções 5.1 .1 a 5.1.4, respectivamente.

\subsubsection{Diretrizes do Esquema}

Por um lado, esquemas existentes na literatura voltados a DWs convencionais que possibilitam a execução de consultas OLAP drill-across, como o esquema do benchmark TPC-H (Figura 2.5), não possuem dados e hierarquias espaciais, impossibilitando a execução de consultas SOLAP de forma geral. Por outro lado, esquemas voltados a DWGs, como o NewGHSSB (Figura 2.9), permitem a execução de consultas SOLAP, mas não especificamente do tipo drill-across, pois possuem apenas uma tabela de fatos. Assim, foi definido nesta dissertação um conjunto de diretrizes que deve ser seguido para o projeto de um esquema de DWG que possibilite a execução de consultas SOLAP drill-across.

O projeto do DWG deve ser desenvolvido com base nas seguintes diretrizes:

- O esquema deve possuir pelo menos uma constelação de fatos, ou seja, deve possuir no mínimo duas tabelas de fatos com dimensões em comum.

- Os dados espaciais devem estar armazenados como atributos em tabelas de dimensão.

- Quando existir uma relação 1:1 entre os elementos de um conjunto de objetos espaciais $\mathcal{E}$ e as entradas de uma tabela de dimensão $\mathcal{T}$, esses objetos espaciais devem ser armazenados como atributos espaciais dessa dimensão $\mathcal{T}$. Caso contrário, esses objetos $\mathcal{E}$ 
devem ser armazenados em uma nova tabela de dimensão espacial, que é referenciada pela tabela de dimensão original $\mathcal{T}$. Em especial, esse princípio está de acordo com o trabalho de Mateus et al. (2010).

Note que a existência de hierarquia de atributos espaciais viabiliza a execução de consultas SOLAP dos tipos drill-down e roll-up em conjunto com consultas SOLAP drill-across. Essa característica é usada nas propostas realizadas para as classes de consultas descritas na seção 5.1.2.

\subsubsection{Classes de Consultas}

De forma similar à discussão realizada na seção 5.1.1, consultas definidas sobre benchmarks de DWs convencionais não possuem predicados espaciais (e.g., TPC-H). Em contrapartida, consultas SOLAP definidas sobre benchmarks de DWGs não são do tipo drill-across por serem executadas sobre apenas uma tabela de fatos (e.g., NewGHSSB). Nessa seção, são propostas três diferentes classes de consultas SOLAP drill-across, as quais enfocam na análise de diferentes tipos de dados espaciais, diferentes granularidades e números crescentes de janelas de consulta espacial, impondo dessa forma diferentes custos de processamento. Essas classes definem o formato das consultas que devem ser executadas sobre um DWG projetado de acordo com as diretrizes definidas na seção 5.1.1.

As classes de consulta propostas nesta dissertação compartilham as seguintes características:

- Utilizam pelo menos duas tabelas de fatos distintas com uma ou mais dimensões compartilhadas.

- Recuperam ou manipulam pelo menos uma medida numérica de cada tabela de fatos.

- Recuperam pelo menos dois atributos convencionais de quaisquer dimensões.

- Possuem ou não predicados convencionais definidos sobre quaisquer dimensões.

- Possuem a cláusula group by definida sobre os atributos convencionais recuperados.

- Possuem a cláusula order by definida sobre os atributos convencionais recuperados.

- Permitem a definição de diferentes consultas, de forma que consultas de uma mesma classe que diferenciam-se somente pelo nível de granularidade sejam organizadas e enumeradas em ordem crescente de granularidade, caracterizando um conjunto de consultas SOLAP roll-up/drill-down.

Além dessas características em comum, cada classe de consulta possui características específicas relacionadas aos seus predicados espaciais, conforme detalhado a seguir. 


\section{Classe Q1}

A classe Q1 engloba consultas que possuam apenas um predicado espacial do tipo range query definido sobre um atributo espacial relacionado a uma dimensão espacial. Consultas da classe Q1 possuem uma janela de consulta espacial que restringe a localização das entradas da tabela de dimensão espacial a uma certa região do espaço. Essa região é determinada de acordo com o relacionamento topológico das localizações dessas entradas e a janela de consulta utilizada. Ademais, as consultas da classe Q1 podem ser organizadas de acordo com a hierarquia de atributos espaciais envolvida, dentro do contexto de consultas SOLAP roll-up/drill-down.

\section{Exemplo 1}

Considere uma hierarquia espacial da dimensão Hospital envolvida no predicado espacial, representado por $\left(H_{\text {região }}\right) \preceq\left(H_{\text {estado }}\right) \preceq\left(H_{\text {cidade }}\right) \preceq\left(H_{\text {rua }}\right)$. Um possível conjunto de consultas roll-up/drill-down da classe Q1, considerando essa hierarquia, é organizado da seguinte forma:

- Q1.1: granularidade rua de Hospital.

- Q1.2: granularidade cidade de Hospital.

- Q1.3: granularidade estado de Hospital.

- Q1.4: granularidade região de Hospital.

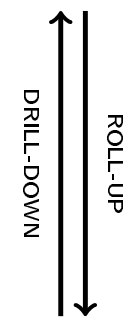

\section{Classe Q2}

A classe Q2 engloba consultas que possuam dois predicados espaciais do tipo range query definidos sobre atributos espaciais relacionados a dimensões espaciais distintas e que estejam no mesmo nível de granularidade.

Consultas da classe Q2 são mais complexas que consultas da classe $Q 1$, pois restringem as localizações de dois conjuntos de entradas pertencentes às dimensões espaciais distintas. Além disso, consultas da classe Q2 realizam mais operações de junção quando comparadas às consultas da classe Q1. De maneira similar à classe Q1, as consultas da classe Q2 podem ser organizadas de acordo com as hierarquias de atributos espaciais envolvidas.

\section{Exemplo 2}

Considere duas hierarquias espaciais, $\left(H_{\text {região }}\right) \preceq\left(H_{\text {estado }}\right) \preceq\left(H_{\text {cidade }}\right) \preceq\left(H_{\text {rua }}\right)$ e $\left(P_{\text {região }}\right) \preceq\left(P_{\text {estado }}\right) \preceq\left(P_{\text {cidade }}\right) \preceq\left(P_{\text {rua }}\right)$, relacionadas a duas dimensões 
espaciais, Hospital e Paciente, envolvidas nos predicados espaciais. Um possível conjunto de consultas roll-up/drill-down dessa classe é:

- Q2.1: granularidades rua de Hospital e rua de Paciente.

- Q2.2: granularidades cidade de Hospital e cidade de Paciente.

- Q2.3: granularidades estado de Hospital e estado de Paciente.

- Q2.4: granularidades região de Hospital e região de Paciente.

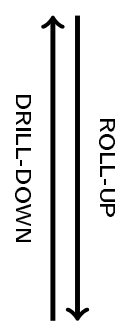

\section{Classe Q3}

A classe Q3 engloba consultas que possuam dois predicados espaciais do tipo range query definidos sobre atributos espaciais relacionados a dimensões espaciais distintas. Além disso, os predicados espaciais incidem sobre níveis de granularidade e tipos de dados espaciais distintos.

Assim como a classe Q2, a classe Q3 restringe as localizações de dois conjuntos de entradas pertencentes a dimensões espaciais distintas. Porém, na classe Q3 essas localizações são de níveis de granularidades diferentes. O objetivo dessa diferença de granularidade é explorar o processamento de diferentes tipos de dados espaciais, como pontos e polígonos, além do processamento de diferentes cardinalidades em uma mesma consulta. Nessa classe também pode ser realizada uma organização baseada nas hierarquias espaciais.

\section{Exemplo 3}

Considere novamente duas hierarquias, $\left(H_{\text {região }}\right) \preceq\left(H_{\text {estado }}\right) \preceq\left(H_{\text {cidade }}\right) \preceq$ $\left(H_{\text {rua }}\right)$ e $\left(P_{\text {região }}\right) \preceq\left(P_{\text {estado }}\right) \preceq\left(P_{\text {cidade }}\right) \preceq\left(P_{\text {rua }}\right)$, relacionadas a duas dimensões espaciais, Hospital e Paciente, envolvidas nos predicados espaciais. Ruas são representadas por linhas, enquanto que cidades, estados e regiões são representados por polígonos. Um possível conjunto de consultas pode ser criado fixando a granularidade de Paciente e variando a granularidade de Hospital. A organização desse conjunto é feita da seguinte forma:

- Q3.1: granularidades cidade de Hospital e rua de Paciente.

- Q3.2: granularidades estado de Hospital e rua de Paciente.

- Q3.3: granularidades região de Hospital e rua de Paciente.

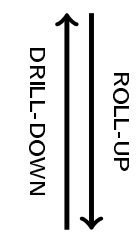

Outro possível conjunto de consultas é formado de forma inversa: a granularidade de Hospital é fixada e a de Paciente é variada, conforme mostrado a seguir: 
- Q3.1: granularidades rua de Hospital e cidade de Paciente.

- Q3.2: granularidades rua de Hospital e estado de Paciente.

- Q3.3: granularidades rua de Hospital e região de Paciente.

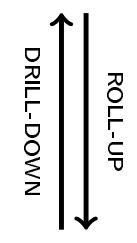

\subsubsection{Estratégia Divide}

Nesta seção é proposta a estratégia Divide, a primeira estratégia desenvolvida para processar consultas SOLAP drill-across utilizando o SB-index. Essa estratégia é baseada em duas etapas principais. Na primeira, cada esquema estrela e cada tabela de dimensão espacial da constelação de fatos é indexada separadamente. Na segunda etapa, cada esquema estrela é processado para produzir subresultados que são combinados, agrupados e ordenados para obter o resultado final.

A ideia que motivou a proposta da estratégia Divide é baseada na discussão que se segue. O Índice Bitmap de junção, empregado na resolução de consultas SOLAP pelo SB-index, considera um esquema estrela de várias tabelas como uma única tabela resultante de todas as operações de junção necessárias para responder uma consulta sobre esse esquema. Isso significa que, para se construir um Índice Bitmap de junção que indexe um atributo de um esquema estrela, é preciso realizar as junções da tabela de fatos com todas as dimensões necessárias, transformando o esquema estrela em uma única tabela. Seguindo essa premissa, a estratégia Divide realiza a construção de vários Índices Bitmap de junção, um para cada esquema estrela da constelação de fatos. Desta forma, o SB-index pode ser aplicado em cada esquema estrela seguindo seu algoritmo original.

Como exibido na Figura 5.1, a qual ilustra o processamento de uma consulta SOLAP drillacross que incide sobre uma constelação de fatos com dois esquemas estrelas, $A$ e $B$, antes da estratégia Divide ser executada, a consulta deve ser dividida em várias subconsultas, cada uma correspondente a um esquema estrela. Cada esquema estrela da constelação de fatos é indexada por um Índice Bitmap de junção $I B J$ (i.e., $I B J_{A}$ e $I B J_{B}$ ), enquanto que cada atributo espacial é indexado por um SB-index $S B$ (i.e., $S B_{1}, S B_{2}, S B_{3}, S B_{4}, S B_{5}$ ). Na sequência, cada subconsulta é processada usando-se os índices apropriados, e os subresultados gerados são combinados, agrupados e ordenados para se obter um resultado final.

Em detalhes, dada uma consulta SOLAP drill-across $Q$, a estrutura de cada subconsulta $S Q_{i}$ é definida da seguinte forma:

- A cláusula SELECT de cada subconsulta $S Q_{i}$ deve possuir os atributos envolvidos nas cláusulas SELECT, GROUP BY e ORDER BY de $Q$ correspondentes ao esquema estrela $E E_{i}$.

- A cláusula FROM de cada subconsulta $S Q_{i}$ deve possuir o esquema estrela $E E_{i}$ correspondente. 


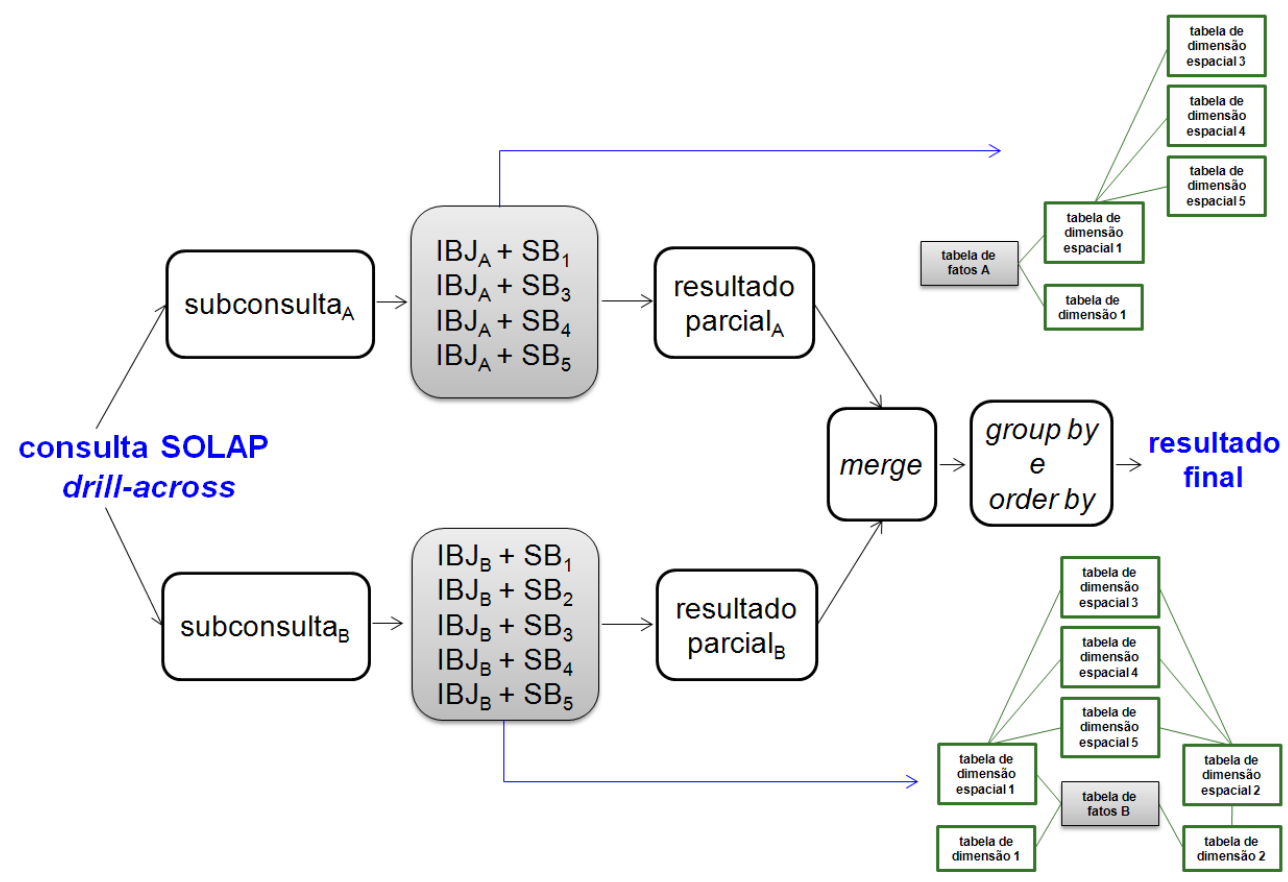

Figura 5.1: Processamento de uma consulta SOLAP drill-across que incide sobre uma constelação de fatos com dois esquemas estrelas, $A$ e $B$, sendo realizado pela estratégia Divide.

- A cláusula WHERE de cada subconsulta $S Q_{i}$ deve possuir os predicados convencionais e espaciais de $Q$ que possam ser aplicados ao esquema estrela $E E_{i}$.

- A cláusula ORDER BY de cada subconsulta $S Q_{i}$ deve possuir os atributos da cláusula SELECT de $S Q_{i}$ ordenados de acordo com os atributos que serão posteriormente utilizados na junção dos subresultados.

Os Algoritmos 1 and 2 detalham a estratégia Divide. O Algoritmo 1, chamado DivideContruirĺndices, define a etapa de construção dos índices, que envolve tanto a construção do Índice Bitmap de junção para cada esquema estrela, como a construção do SB-index para cada tabela de dimensão espacial. O algoritmo recebe como entrada uma constelação de fatos $C F$, com $m$ esquemas estrela e $n$ tabelas de dimensão espacial. Para cada esquema estrela $\left(E E_{i} \in C F\right)$ é criado um Índice Bitmap de junção $I B J_{i}$ (linhas 1 e 2), e para cada tabela de dimensão espacial $T D E_{j}$ é criado um SB-index $S B_{j}$ (linhas 3 e 4 ). Como saída são gerados $m$ Índices Bitmap de junção $\left(I B J_{1}, \ldots, I B J_{m}\right)$ e $n$ SB-index $\left(S B_{1}, \ldots, S B_{n}\right)$. Cada índice $I B J_{i}$ deve conter os atributos correspondentes ao seu $E E_{i}$ envolvidos nas consultas que são executadas. Os SB-index são construídos seguindo o algoritmo de construção original descrito na seção 4.4.1.

O Algoritmo 2, chamado DivideProcessarConsulta, define o processamento de consultas da estratégia Divide. O algoritmo recebe como entrada uma consulta SOLAP drill-across $Q$, uma constelação de fatos $C F$, um conjunto de Índices Bitmap de junção $I B J$ e um conjunto 


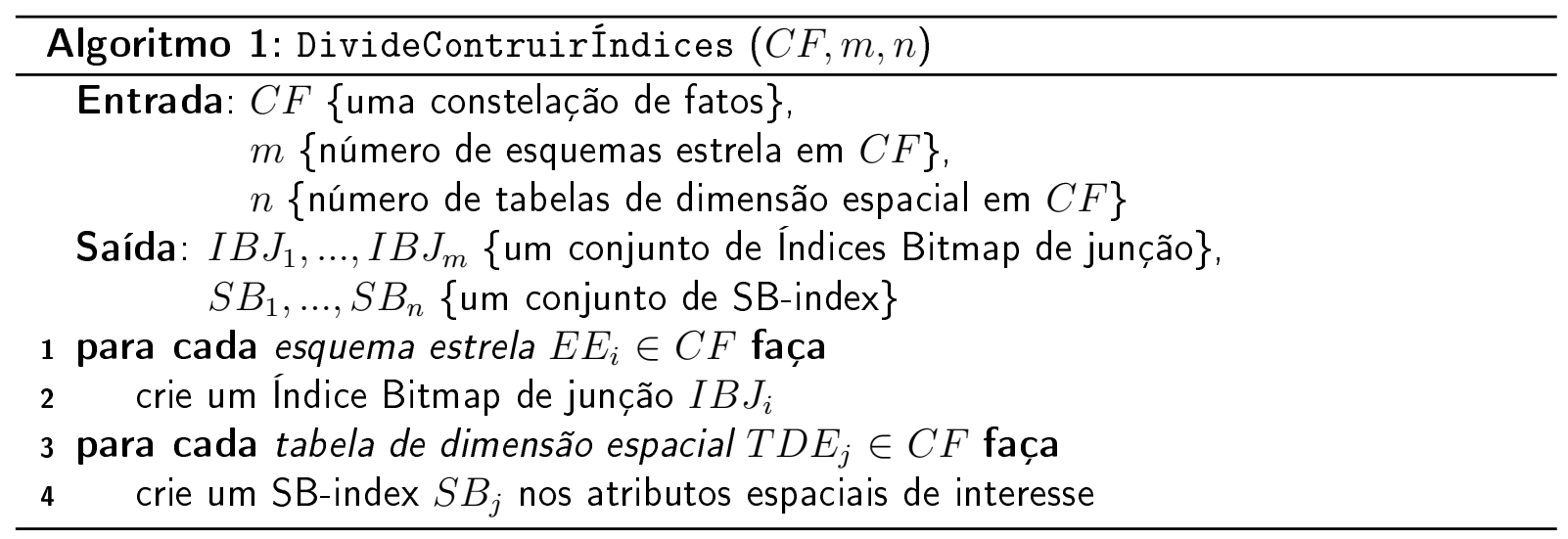

de SB-index $S B$. A consulta $Q$ é subdividida em $m$ subconsultas $S Q_{i}$, onde $m$ é o número de esquemas estrela envolvidos na consulta $Q$ (linhas 1 e 2). Em seguida, $m$ resultados parciais são gerados a partir da aplicação do SB-index em cada subconsulta $S Q_{i}$ (linha 3). O resultado final da consulta SOLAP drill-across, saída do algoritmo, é obtido combinando-se esses resultados parciais por meio das dimensões compartilhadas pelos esquemas estrela (linha 4) e aplicando-se as operações de group by (linha 5) e order by (linha 6) de $Q$. Note que a presença da constelação de fatos como entrada do Algoritmo 2 é necessária para que as tabelas de dimensão espaciais sejam acessadas na fase de refinamento do predicado espacial das subconsultas. O processamento das subconsultas pelo SB-index é realizado utilizando seu algoritmo original descrito na seção 4.4.2.

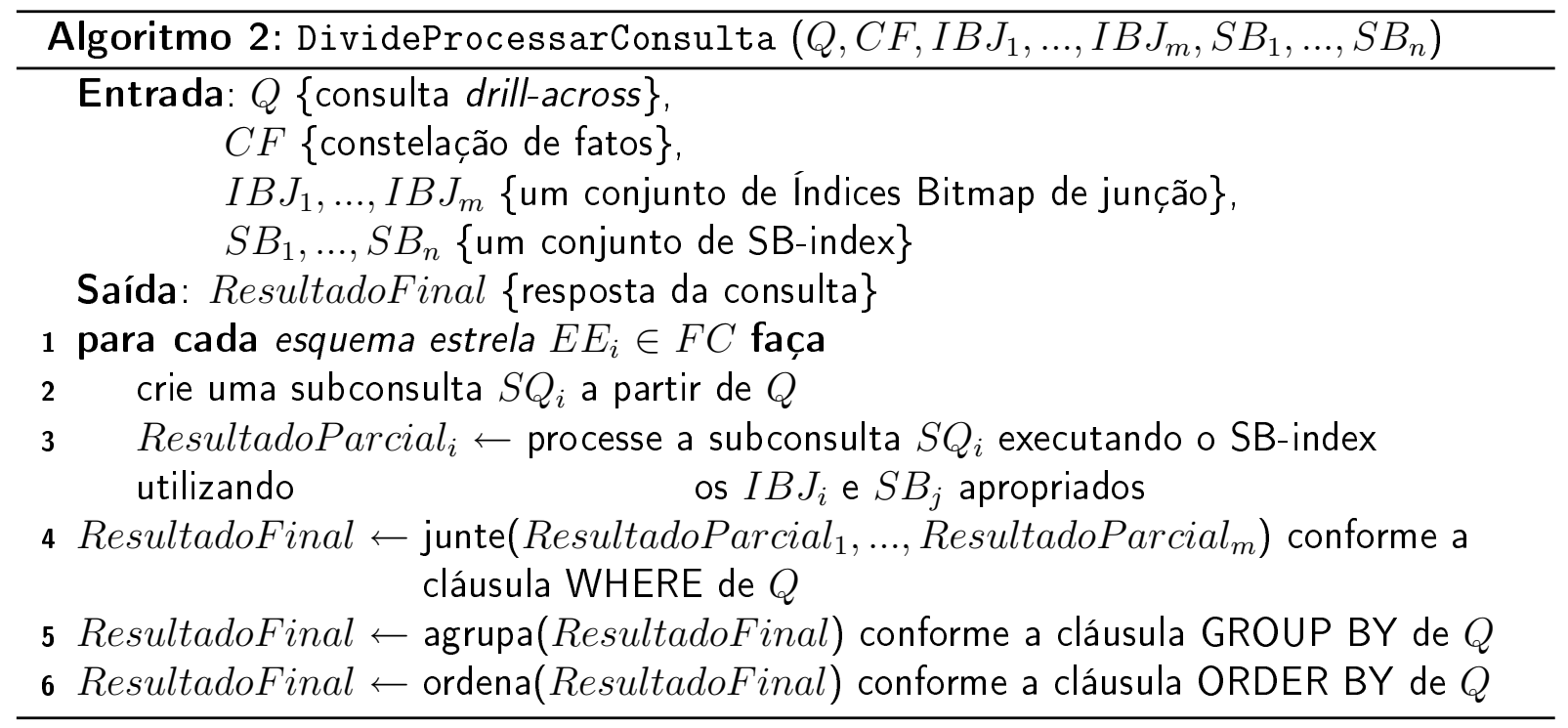

\subsubsection{Estratégia Única}

Nessa seção é definida a segunda estratégia para processar consultas SOLAP drill-across utilizando o SB-index. A estratégia proposta, chamada de Única, também é baseada em duas etapas. Na primeira, a constelação de fatos é indexada por um único Índice Bitmap de junção 
e cada tabela de dimensão espacial da constelação de fatos é indexada por um SB-index. Na segunda etapa, a constelação de fatos é processada para produzir um único subresultado que é agrupado e ordenado para gerar o resultado final.

Assim como a estratégia Divide, a ideia que motivou a proposta da estratégia Única também baseia-se na premissa de que o Índice Bitmap de junção, empregado pelo SB-index, considera um esquema estrela de várias tabelas como uma única tabela resultante de todas as operações de junção necessárias para responder uma consulta. Consequentemente, se a constelação de fatos for considerada como uma única tabela que possua todos os atributos necessários para processar a consulta, é possível construir apenas um único Índice Bitmap de junção que indexe essa constelação de fatos. Como resultado, o SB-index pode ser aplicado da mesma forma que tem sido aplicado em apenas um esquema estrela.

A Figura 5.2 ilustra uma consulta SOLAP drill-across que incide sobre uma constelação de fatos e que é processada pela estratégia Única. É gerado um único Índice Bitmap de junção $I B J$ que indexa a constelação de fatos e é produzido um único subresultado, o qual é agrupado e ordenado para obter o resultado final da consulta. Note que, também para a estratégia Única, cada atributo espacial é indexado por um SB-index $S B$ (i.e., $S B_{1}, S B_{2}$, $\left.S B_{3}, S B_{4}, S B_{5}\right)$.

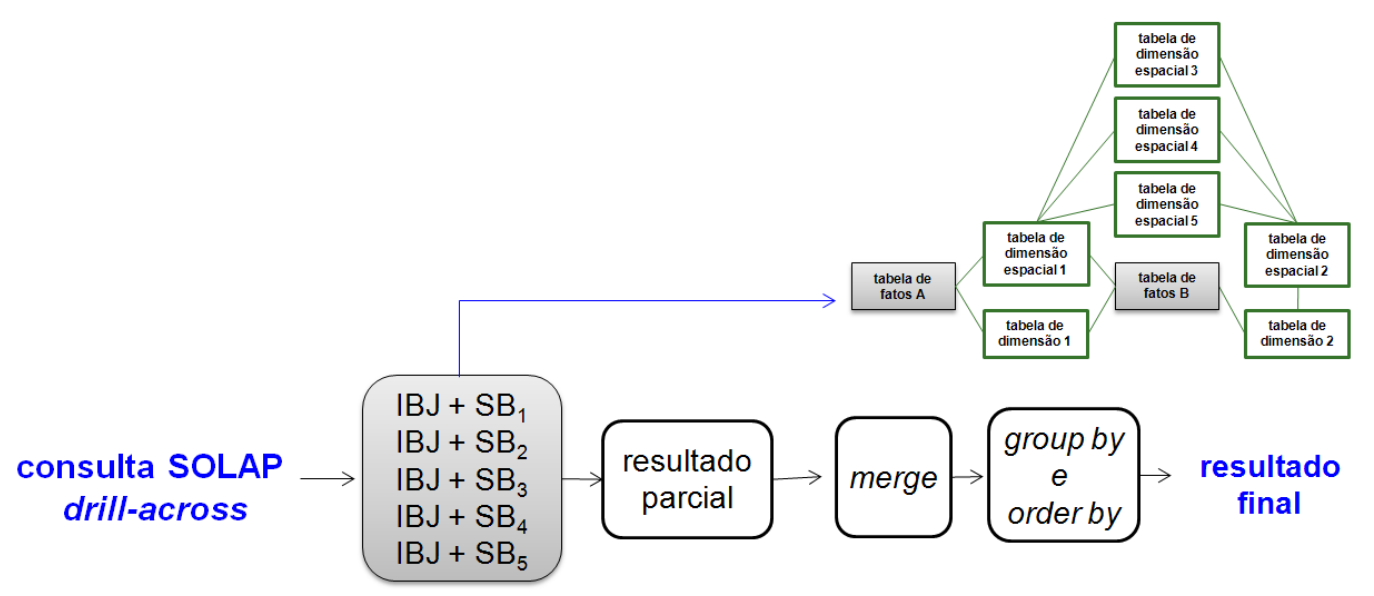

Figura 5.2: Processamento de uma consulta SOLAP drill-across que incide sobre uma constelação de fatos sendo realizado pela estratégia Única.

Os algoritmos 3 e 4 detalham a estratégia Única. O Algoritmo 3, chamado ÚnicaContruirÍndices, define a etapa de construção dos índices, a qual envolve tanto a construção do Índice Bitmap de junção, quanto a construção do SB-index para cada tabela de dimensão espacial. O algoritmo recebe como entrada uma constelação de fatos $C F$ com $n$ tabelas de dimensão espacial. É criado um único Índice Bitmap de junção $I B J$ (linha 1), e para cada tabela de dimensão espacial é criado um SB-index (linhas 2 e 3). Como saída são gerados um único Índice Bitmap de junção $(I B J)$ e $n$ SB-index $\left(S B_{1}, \ldots, S B_{n}\right)$. O índice $I B J$ deve conter todos os atributos de $C F$ envolvidos na consulta que está sendo processada. Os SB-index são construídos seguindo o algoritmo de construção original descrito na seção 4.4.1. 


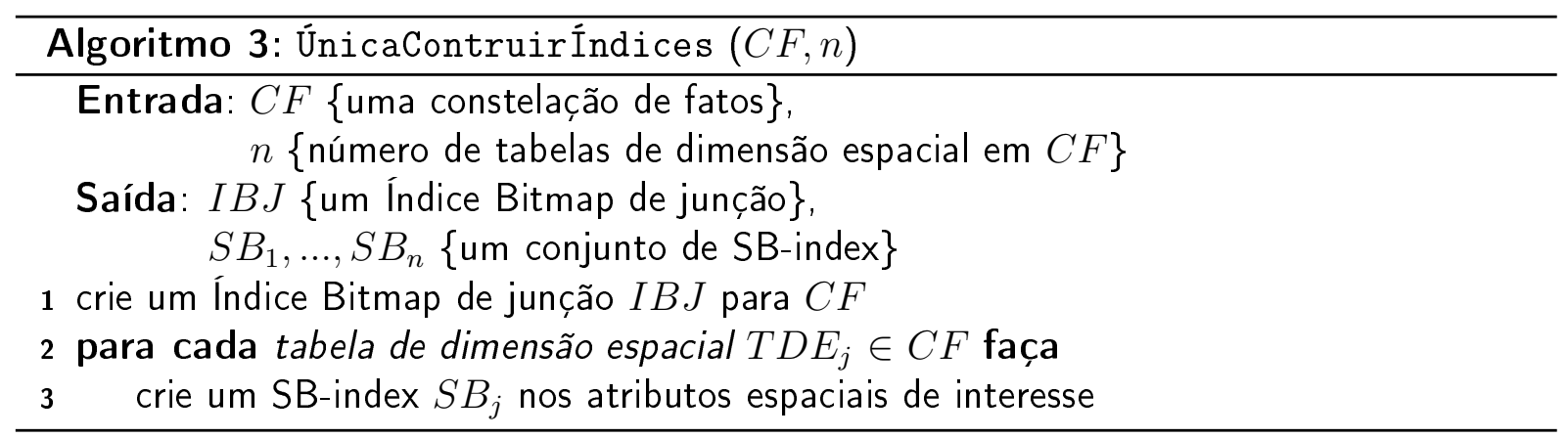

O Algoritmo 4, chamado ÚnicaProcessarConsulta, define o processamento de consultas da estratégia Única. O algoritmo recebe como entrada uma consulta SOLAP drill-across $Q$, uma constelação de fatos $C F$, um Índice Bitmap de junção $I B J$ e um conjunto de SB-index $S B$. Um único resultado parcial é gerado a partir da aplicação do SB-index na consulta $Q$ (linha 1). O resultado final da consulta SOLAP drill-across, saída do algoritmo, é obtido aplicando-se as operações de group by (linha 2) e order by (linha 3) de $Q$. O processamento da consulta pelo SB-index é realizado utilizando seu algoritmo original descrito na seção 4.4.2.

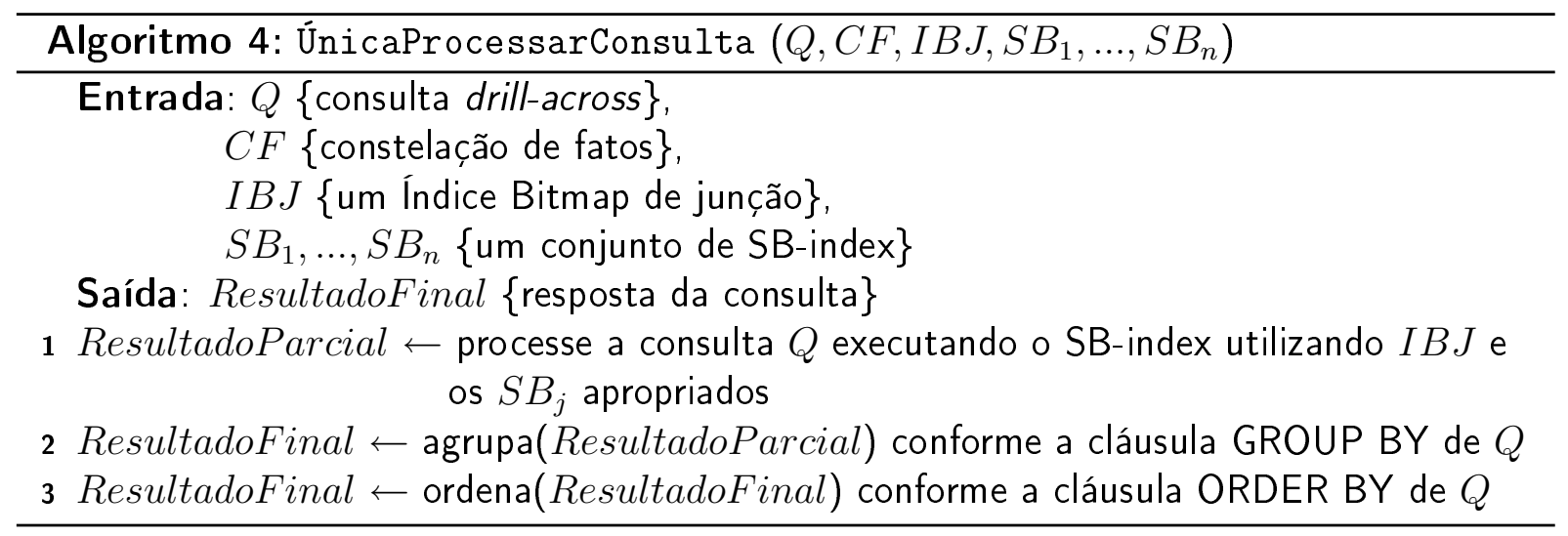

\subsection{Testes de Desempenho}

Nessa seção são apresentados os resultados obtidos nos testes de desempenho do processamento de consultas SOLAP drill-across utilizando as duas estratégias de processamento propostas. Antes da apresentação dos resultados, a seção 5.2.1 detalha a configuração dos testes.

\subsubsection{Configuração dos Testes}

Os seguintes aspectos relacionados à configuração dos testes foram definidos para a execução dos testes de desempenho: ambiente de testes, esquemas, dados, consultas, janelas de consulta, e os métodos usados para comparação. 


\section{Ambiente de Testes}

Os testes foram conduzidos em uma máquina com um processador Intel Core i7 2,67 GHz, 12GB de memória RAM, 2 discos rígidos de 1TB, 2 discos rígidos de 2TB, Linux Ubuntu 11.10, kernel 3.0.0-13-generic-pae, PostgreSQL 9.1.1, PostGIS 1.5.3 e FastBit 1.2.4.

\section{Esquemas}

Com base no conjunto de diretrizes definidas na seção 5.1.1, foi projetado o esquema SpatialDrillAcross, o qual possui dimensões e dados convencionais e espaciais e permite que consultas SOLAP drill-across sejam processadas sobre seus dados. A Figura 5.3 ilustra o esquema proposto, o qual consiste em uma adaptação do esquema do benchmark TPC-H (Pöss e Floyd, 2000) e do NewGHSSB (Mateus et al., 2010). O esquema atende às diretrizes propostas devido às características descritas a seguir.

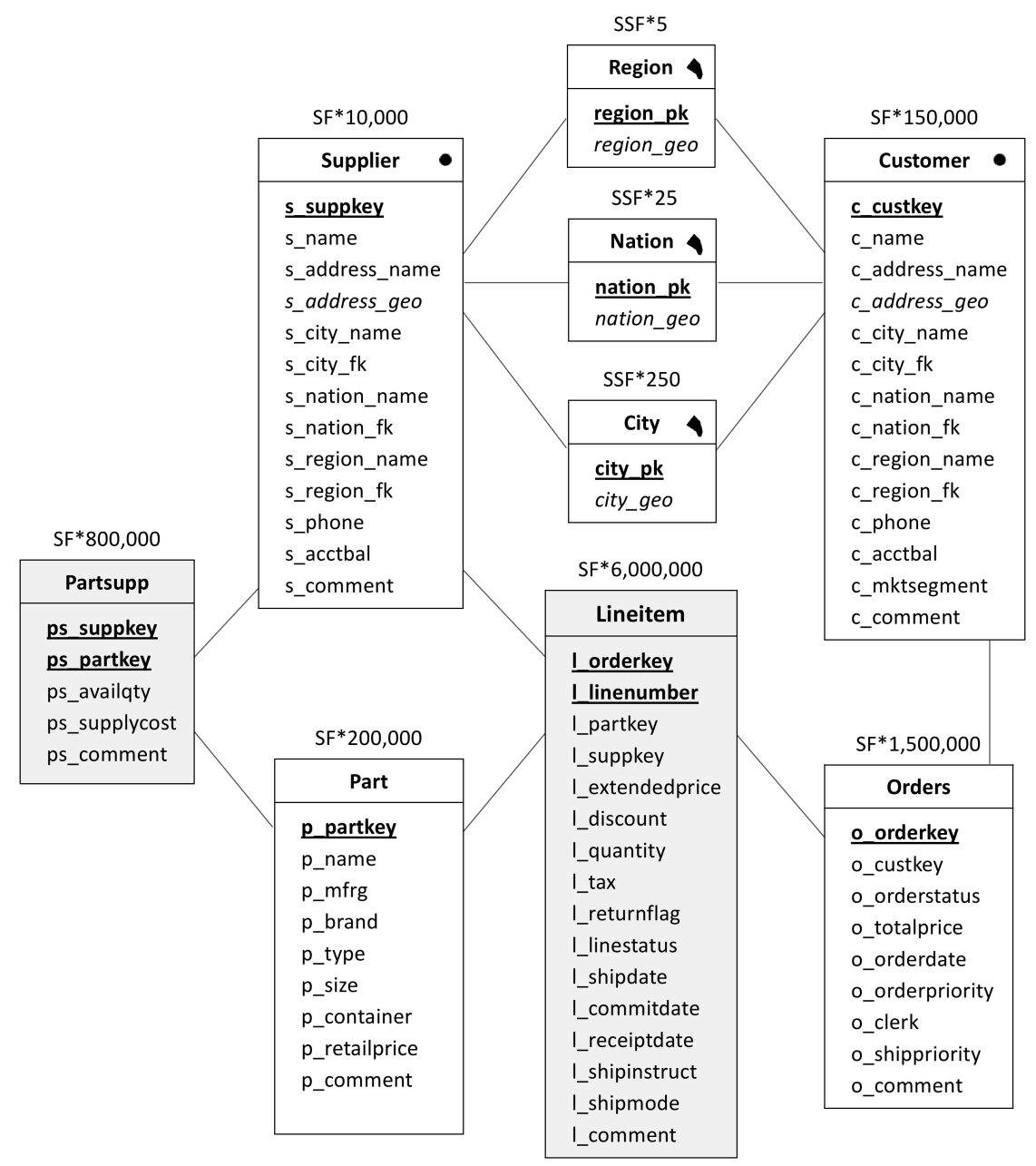

Figura 5.3: Esquema SpatialDrillAcross construído a partir de uma adaptação dos esquemas do benchmark TPC-H e do NewGHSSB . 
- O esquema proposto possui uma constelação de fatos composta pelas tabelas de fatos Lineitem e Partsupp, as quais compartilham as dimensões Part e Supplier.

- Os dados espaciais, identificados pelo sufixo geo, estão armazenados em tabelas de dimensão, mais especificamente nas tabelas de dimensão Supplier, Customer, City, Nation e Region. Esses dados foram introduzidos respeitando a correspondência com os atributos descritivos/convencionais já existentes no TPC-H, exceto City. Além disso, foi introduzido um fator de escala espacial $S S F$ que controla a cardinalidade das tabelas de dimensão espacial City, Nation e Region.

- Os conjuntos de objetos espaciais s_address_geo e c_address_geo, os quais representam endereços de fornecedores e clientes, possuem relações 1:1 com as tabelas de dimensão Supplier e Customer, respectivamente. Portanto, esses conjuntos de objetos espaciais estão armazenados como atributos espaciais nas tabelas Supplier e Customer, respectivamente. Além disso, Customer e Supplier não compartilham endereços semelhantes.

- Existem duas hierarquias espaciais predefinidas do tipo simétrico simples com o relacionamento topológico está contido: $(i)($ region_geo $) \preceq($ nation_geo $) \preceq($ city_geo $) \preceq$ (c_address_geo); e $($ ii) $($ region_geo $) \preceq($ nation_geo $) \preceq($ city_geo $) \preceq($ s_address_geo $)$. Isso significa que uma geometria em um atributo de granularidade espacial de nível mais baixo está contida por somente uma geometria em um atributo de granularidade espacial de nível mais alto. A dimensão espacial City foi incorporada ao esquema proposto, adicionando mais um grau de granularidade espacial, embora não exista no TPC-H um atributo descritivo/convencional correspondente.

Adicionalmente, foi gerado um segundo esquema a partir de modificações nas tabelas de fatos Lineitem e Partsupp do esquema SpatialDrillAcross. Esse esquema não possui significado semântico, pois visa apenas permitir comparações entre as estratégias de processamento Divide e Única, ou seja, visa permitir que diferentes proporções entre as tabelas de fatos Lineitem e Partsupp sejam exploradas pelas estratégias propostas. A Figura 5.4 ilustra o esquema SpatialDrillAcross modificado.

Em detalhes, na tabela Lineitem foi introduzido o parâmetro $n$ para controlar a proporção de tuplas que essa tabela mantém com a tabela de fatos Partsupp. Cada tupla da tabela Lineitem sempre realiza junção com apenas uma tupla da tabela Partsupp. Além do parâmetro $n$, foram introduzidos dois outros atributos: $l_{-} n$ e $l_{-} i d$. $\mathrm{O}$ atributo $l_{-} n$ compõe a chave primária e possui um valor inicial igual a 1 . Esse valor é acrescido de uma unidade toda vez que a tabela é replicada para aumentar essa proporção. A função desse atributo é garantir a integridade da chave primária quando seus dados são replicados. Por exemplo, se o parâmetro $n$ for igual a 3 , os possíveis valores do atributo $l_{-} n$ são 1,2 e 3 . A outra modificação refere-se à introdução do atributo $l_{-} i d$, que também compõe a chave primária e que é usado na cláusula de junção entre as tabelas de fatos. Seu objetivo é garantir a proporção correta entre as tuplas das tabelas 


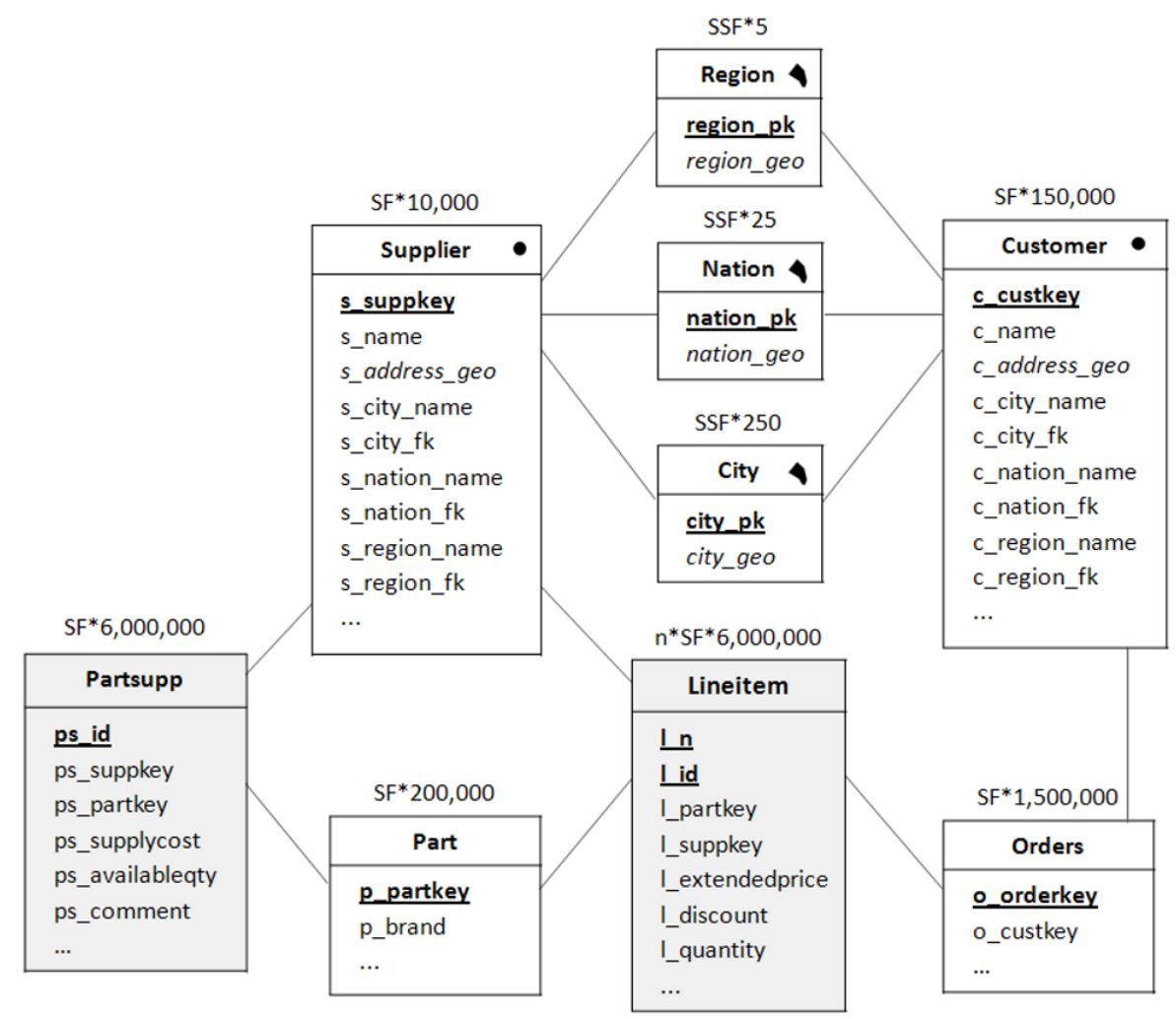

Figura 5.4: Esquema SpatialDrillAcross modificado.

de fatos quando a operação de junção é executada. Na tabela Partsupp foi introduzido o atributo $p s \_i d$, o qual relaciona as tuplas da tabela Partsupp com as tuplas da tabela Lineitem por meio do atributo $l_{-} i d$. Consequentemente, houve um aumento na quantidade de tuplas da tabela Partsupp.

A tabela de fatos Partsupp é gerada a partir da replicação de alguns atributos da tabela $\mathrm{Li}$ neitem quando $l_{-} n=1$. Os atributos $l_{-} i d, l_{-}$suppkey, $l \_$partkey, $l_{-}$extendedprice, $l_{-} q u a n t i t y$ e $l_{-}$comment da tabela Lineitem se transformam nos atributos ps_id, ps_suppkey, ps_partkey, ps_supplycost, ps_availqty e ps_comment, respectivamente, considerando $l_{-} n=1$. Nessa forma de gerar a tabela de fatos Partsupp, é mantido o mesmo tipo de dados dos atributos ps_supplycost e ps_availqty, mas não há uma preocupação com o domínio em si, desde que esses atributos não compõem predicados nas consultas, e por consequência, não afetam a seletividade das consultas e o volume de dados retornado pelas mesmas. A geração dos dados da tabela Lineitem para valores $l_{-} n>1$ é realizada por meio da replicação de seus dados quando $l_{-} n=1$. Essa forma de gerar essas duas tabelas foi escolhida visando manter a distribuição dos dados da tabela Lineitem gerada pelo benchmark TPC-H. Assim, a seletividade de um predicado que atue sobre a tabela Lineitem é mantida nos dois esquemas propostos e é possível gerar bases com diferentes proporções.

\section{Dados}


O dados convencionais e espaciais utilizados são dados sintéticos. Dados convencionais foram gerados a partir do benchmark TPC-H e dados espaciais foram gerados a partir do benchmark Spadawan. A cardinalidade das tabelas de dimensão espacial City, Nation e Region é controladada pelo fator de escala espacial, $S S F$. Este parâmetro permite explorar como o desempenho das estratégias de processamento são afetadas mediante a um fator de escala convencional fixo. Os dados espaciais originais do benchmark Spadawan possuem 250 cidades, 25 nações e 5 regiões, todos representados por polígonos. O aumento desse volume de dados é obtido por meio da replicação e translação do conjunto original de dados de modo que não haja sobreposição, gerando $S S F * 250$ cidades, $S S F * 25$ nações e $S S F * 5$ regiões. A Figura 5.5 ilustra os dados da tabela de dimensão espacial Region replicados a partir do conjunto original de 5 regiões. Dados espaciais referentes aos endereços de fornecedores e clientes, representados por pontos, seguem a cardinalidade das tabelas Supplier e Customer, respec de acordo com as diretrizes definidas na seção 5.1.1, tivamente. Isso corresponde a $S F * 10.000$ endereços na tabela Supplier e a $S F * 150.000$ endereços na tabela Customer.
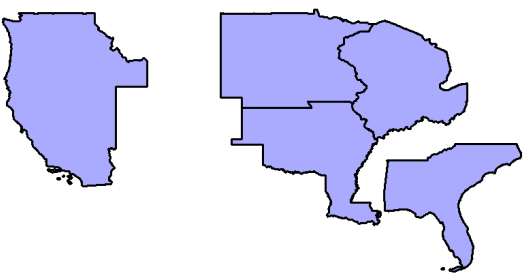

(a) Conjunto original - $S S F=1$.
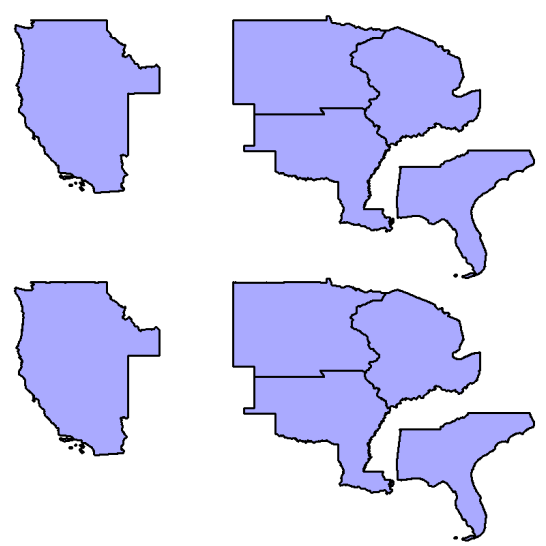

(b) Conjunto replicado $-S S F=2$.

Figura 5.5: Dados da tabela de dimensão espacial Region.

Com relação aos dados convencionais, no esquema SpatialDrillAcross as tabelas de fatos Partsupp e Lineitem possuem $S F * 800.000$ e $S F * 6.000 .000$ tuplas, respectivamente. Já no esquema SpatialDrillAcross modificado, as tabelas de fatos Partsupp e Lineitem possuem $S F * 6.000 .000$ e $n * S F * 6.000 .000$ tuplas, respectivamente.

\section{Consultas}

As Figuras 5.6 e 5.7 ilustram as consultas utilizadas nos testes de desempenho. Essas consultas compõem três grupos, os quais são baseados nas três classes de consultas definidas na seção 5.1.2. A Figura 5.6 ilustra o template das consultas, enquanto a Figura 5.7 detalha os valores das variáveis granularidade e predicado espacial para cada consulta. As janelas de consultas são representadas por $Q W_{D g}$, onde os subíndices $D$ e $g$ indicam a dimensão e 
granularidade na qual a janela de consulta é aplicada, respectivamente. De acordo com o template, todas as consultas compartilham as seguintes características:

- Utilizam as tabelas de fatos Partsupp e Lineitem.

- Manipulam três medidas numéricas, l_discount, l_extendedprice e l_quantity, da tabela de fatos Lineitem, e a medida numérica ps_supplycost da tabela de fatos Partsupp.

- Recuperam dois atributos convencionais: o_year e um atributo determinado pela variável granularidade, relacionado com a granularidade da consulta.

- Possuem a cláusula group by sobre os atributos convencionais recuperados.

- Possuem a cláusula order by sobre os atributos convencionais recuperados.

As consultas Q1.1, Q1.2, Q1.3 e Q1.4 fazem parte da classe Q1. Elas possuem um predicado espacial do tipo range query sobre a dimensão espacial Supplier, restringindo a localização de fornecedores a uma certa região do espaço, de acordo com o relacionamento topológico desses fornecedores e a janela de consulta $Q W_{D g}$. A consulta Q1.1 possui um predicado espacial sobre a granularidade (s_address_geo) da hierarquia espacial de Supplier e recupera o atributo convencional s_name. Nas consultas Q1.2 e Q1.3, o predicado espacial incide sobre as granularidades (city_geo) e (nation_geo), respectivamente, da hierarquia espacial de Supplier. Além disso, as consultas Q1.2 e Q1.3 recuperam os atributos convencionais s_city_name e $s_{-} n a t i o n \_n a m e$, respectivamente. A consulta Q1.4 possui um predicado espacial sobre a granularidade (region_geo) da hierarquia espacial de Supplier e recupera o atributo convencional s_region_name. Essas quatro consultas constituem um conjunto de operações roll-up/drill-down.

As consultas Q2.1, Q2.2, Q2.3 e Q2.4 fazem parte da classe Q2 e estão relacionadas com as duas hierarquias espaciais presentes no esquema SpatialDrillAcross. Elas possuem dois predicados espaciais do tipo range query, cada um relacionado a uma hierarquia. Em cada consulta, ambos os predicados estão no mesmo nível de granularidade. Essas consultas são mais complexas, quando comparadas nos níveis correspondentes, que as consultas da classe Q1, pois além de restringirem as localizações dos fornecedores e as localizações dos clientes, também realizam mais operações de junção. A consulta Q2.1 possui dois predicados espaciais, um sobre a granularidade (s_address_geo) da hierarquia espacial de Supplier e outro sobre a granularidade (c_address_geo) da hierarquia espacial de Customer, e recupera o atributo convencional s_name. A consulta Q2.2 possui dois predicados espaciais, ambos sobre a granularidade (city_geo) das hierarquias espaciais de Supplier e Customer, e recupera o atributo convencional s_city_name. A consulta Q2.3 possui dois predicados espaciais, ambos sobre a granularidade (nation_geo) das hierarquias espaciais de Supplier e Customer, e recupera o atributo convencional s_nation_name. Por fim, a consulta Q2.4 possui dois predicados 


\section{SELECT granularidade,}

extract(year from o_orderdate) as o_year,

SUM(I_extendedprice*(1 - I_discount) - I_quantity*ps_supplycost) as revenue

FROM Partsupp, Part, Supplier, Lineitem, Orders, tabelas de dimensão espacial

WHERE ps_partkey = p_partkey

AND ps_suppkey $=$ s_suppkey $_{-}$

AND I_partkey = p_partkey

AND I_suppkey $=$ s_suppkey

AND I_orderkey = o_orderkey

AND junções necessárias de acordo com o predicado espacial

AND predicado espacial

GROUP BY granularidade, o_year

ORDER BY granularidade, o_year

Figura 5.6: Template das classes de consultas SOLAP drill-across.

\begin{tabular}{|c|l|l|}
\hline Consulta & granularidade & \multicolumn{1}{|c|}{ predicado espacial } \\
\hline Q1.1 & s_name & WITHIN $\left(s \_a d d r e s s \_g e o, Q W_{S a}\right)$ \\
\hline Q1.2 & $s_{-}$city_name & INTERSECTS $\left(\right.$city_geo, $\left.Q W_{S c}\right)$ \\
\hline Q1.3 & s_nation_name & INTERSECTS $\left(\right.$ nation_geo, $\left.Q W_{S n}\right)$ \\
\hline Q1.4 & s_region_name & INTERSECTS $\left(\right.$ region_geo, $\left.Q W_{S r}\right)$ \\
\hline
\end{tabular}

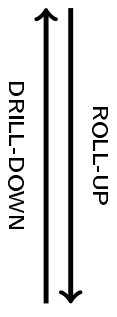

\begin{tabular}{|c|l|l|}
\hline Consulta & granularidade & predicado espacial \\
\hline Q2.1 & $s_{-} n a m e$ & WITHIN $\left(s \_a d d r e s s \_g e o, Q W_{S a}\right)$ and WITHIN $\left(c \_a d d r e s s \_g e o, Q W_{C a}\right)$ \\
\hline Q2.2 & $s_{-}$city_name & INTERSECTS $\left(\right.$city_geo, $\left.Q W_{S c}\right)$ and INTERSECTS $\left(\right.$ city_geo, $\left.Q W_{C c}\right)$ \\
\hline Q2.3 & $s_{-} n a t i o n \_n a m e$ & INTERSECTS $\left(\right.$ nation_geo, $\left.Q W_{S n}\right)$ and INTERSECTS $\left(\right.$ nation_geo, $\left.Q W_{C n}\right)$ \\
\hline Q2.4 & s_region_name & INTERSECTS $\left(\right.$ region_geo, $\left.Q W_{S r}\right)$ and INTERSECTS $\left(\right.$ region_geo, $\left.Q W_{C r}\right)$ \\
\hline
\end{tabular}

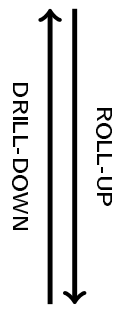

\begin{tabular}{|c|l|c|}
\hline Consulta & granularidade & predicado espacial \\
\hline Q3.1 & $s_{-}$city_name & INTERSECTS $\left(\right.$city_geo, $\left.Q W_{S c}\right)$ and WITHIN $\left(\right.$ address_geo, $\left.Q W_{C a}\right)$ \\
\hline Q3.2 & s_nation_name & INTERSECTS $\left(\right.$ nation_geo, $\left.Q W_{S n}\right)$ and WITHIN $\left(\right.$ address_geo, $\left.Q W_{C a}\right)$ \\
\hline Q3.3 & s_region_name & INTERSECTS $\left(\right.$ region_geo, $\left.Q W_{S r}\right)$ and WITHIN $\left(\right.$ address_geo, $\left.Q W_{C a}\right)$ \\
\hline
\end{tabular}

Figura 5.7: Valores de granularidade e predicado espacial paras as classes Q1, Q2 e Q3. 
espaciais, ambos sobre a granularidade (region_geo) das hierarquias espaciais de Supplier e Customer, e recupera o atributo convencional s_region_name.

As consultas Q3.1, Q3.2 e Q3.3 fazem parte da classe Q3 e também estão relacionadas com as duas hierarquias espaciais presentes no esquema SpatialDrillAcross. Elas possuem dois predicados espaciais do tipo range query, cada um relacionado a uma hierarquia. Diferentemente da classe Q2, na qual em cada consulta ambos os predicados estão em níveis diferentes de granularidade, nas consultas da classe Q3 o predicado espacial relacionado à hierarquia espacial de Customer é mantido fixo na granularidade (c_address_geo) e apenas a granularidade do predicado espacial relacionado à hierarquia espacial da dimensão Supplier é variado. O objetivo dessa configuração, como já destacado anteriormente, é explorar diferentes tipos de dados, pontos e polígonos, e diferentes cardinalidades na mesma consulta. As consultas dessa classe, assim como a classe Q2, restringem as localizações dos fornecedores e clientes. A consulta Q3.1 possui um predicado espacial sobre a granularidade (city_geo) da hierarquia espacial de Supplier e outro predicado espacial sobre a granularidade (c_address_geo) da hierarquia espacial de Customer, e recupera o atributo convencional s_city_name. A consulta Q3.2 possui um predicado espacial sobre a granularidade (nation_geo) da hierarquia espacial de Supplier e outro sobre a granularidade (c_address_geo) de Customer, e recupera o atributo convencional s_nation_name. Por fim, a consulta Q3.3 possui um predicado espacial sobre a granularidade (region_geo) da hierarquia espacial de Supplier e outro sobre a granularidade (c_address_geo) de Customer, recuperando o atributo convencional s_region_name.

\section{Janelas de Consulta}

Nos testes de desempenho foram utilizados cinco conjuntos de janelas de consulta, quatro nas localizações de fornecedores e um nas localizações de clientes. Para cada base de dados utilizada, cada conjunto é formado por quatro janelas concêntricas de formato quadrado, uma para cada nível de granularidade das hierarquias espaciais de Supplier e de Customer. Portanto, cada base utilizada possui seus próprios conjuntos. Durante a exibição dos resultados, quando for indicado que foi utilizado o conjunto $Q W_{S 1}$ em um teste, significa que em cada base foram utilizadas janelas que seguem a seletividade desse conjunto nas respectivas granularidades.

Na Tabela 5.1 são detalhadas as seletividades para cada nível de granularidade dos cinco conjuntos de janelas de consulta. Os subíndices $S$ e $C$ correspondem às dimensões Supplier e Customer, respectivamente.

\section{Métodos para Comparação}

Os métodos usados para comparar e validar as estratégias propostas foram a junção estrela e visões materializadas. Em especial, o índice espacial R-tree foi utilizado para indexar os dados espaciais tanto da junção estrela quando das visões materializadas. Na contrução do índice Bitmap de junção pelo software FastBit, foram utilizadas as opções <binning none/> 
Tabela 5.1: Seletividade dos Conjuntos de Janelas de Consulta.

\begin{tabular}{|c|c|c|c|c|c|}
\hline \multirow{2}{*}{ Granularidade } & \multicolumn{5}{|c|}{ Conjuntos de janelas de consulta } \\
\cline { 2 - 6 } & $Q W_{S 1}$ & $Q W_{S 2}$ & $Q W_{S 3}$ & $Q W_{S 4}$ & $Q W_{C 1}$ \\
\hline Endereço & $0,04 \%$ & $1,22 \%$ & $2,40 \%$ & $3,58 \%$ & $1,00 \%$ \\
\hline Cidade & $0,20 \%$ & $2,40 \%$ & $4,60 \%$ & $6,80 \%$ & $1,00 \%$ \\
\hline Nação & $0,40 \%$ & $3,20 \%$ & $6,00 \%$ & $8,80 \%$ & $1,60 \%$ \\
\hline Região & $2,00 \%$ & $6,00 \%$ & $10,00 \%$ & $14,00 \%$ & $2,00 \%$ \\
\hline
\end{tabular}

e <encoding equality/>. Isto significa que a técnica de otimização binning não foi utilizada e que foi contruído um vetor Bitmap para cada valor de todos os atributos.

\section{Medida Coletada}

Nos testes de desempenho foi coletado o tempo gasto em segundos na execução das consultas. O tempo de cada consulta corresponde à média de 5 execuções, sendo que entre cada execução foi realizada a limpeza da memória cache.

\subsubsection{Comparação entre Junção Estrela, Visão Materializada e as Estratégias Divide e Única}

O objetivo desse teste foi comparar o desempenho das estratégias propostas Divide e Única com o uso da junção estrela e de visões materializadas. As visões materializadas consideradas no teste foram as visões utilizadas na criação dos índices Bitmap de junção pelas duas estratégias propostas. A estratégia Divide utiliza duas visões materializadas, representadas por $\mathrm{V}_{D}$, uma para cada esquema estrela, enquanto que a estratégia Única utiliza apenas uma visão materializada, a qual é representada por $\mathrm{V}_{U}$.

Adicionalmente, foi utilizada uma base fixa gerada a partir do esquema SpatialDrillAcross com a seguinte configuração: $S F=10$ e $S S F=10$. O conjunto de janelas de consulta $Q W_{S 1}$ foi utilizado nas localizações dos fornecedores e nas dos clientes, $Q W_{C 1}$.

Os resultados desse teste de desempenho são exibidos na Figura 5.8, onde cada gráfico corresponde a uma consulta. Os gráficos mostram que as estratégias Divide e Única sempre garantiram desempenho superior quando comparadas com o desempenho da junção estrela e das visões $\mathrm{V}_{D}$ e $\mathrm{V}_{U}$ em todas as consultas. A junção estrela apresentou o pior desempenho. A estratégia Única mostrou uma redução no tempo de $82,7 \%$ a $97,7 \%$ quando comparada ao uso da junção estrela, e de $75,8 \%$ a $90,5 \%$ quando comparada ao uso das visões $\bigvee_{D}$ e $\bigvee_{U}$. 
A estratégia Divide mostrou uma redução no tempo de $87,7 \%$ a $98,6 \%$ quando comparada ao uso da junção estrela, e de $82,7 \%$ a $93,9 \%$ quando comparada ao uso das visões $\mathrm{V}_{D}$ e $V_{U}$. Portanto, as duas estratégias propostas são excelentes opções como estratégias de processamento para consultas SOLAP drill-across.

Analisando-se os resultados obtidos, pode-se verificar que as duas estratégias propostas superam muito o desempenho da junção estrela e das visões materializadas, confirmando as expectativas de que o uso do SB-index, o qual, segundo o nosso conhecimento, é o índice para DWG mais rápido existente na literatura, foi uma boa opção empregada nas estratégias propostas.

Os testes de desempenho que se seguem visam comparar as estratégias Divide e Única considerando diferentes cenários, com o objetivo de identificar em quais cenários cada estratégia é melhor. Portanto, nos próximos testes, não são ilustrados resultados de desempenho para a junção estrela e as visões materializadas.

\subsubsection{Divide e Única: Variando a Seletividade das Consultas}

O objetivo deste teste foi comparar o desempenho entre as estratégias Divide e Única quando a seletividade das consultas é variada. A variação da seletividade é realizada por meio da utilização de diferentes conjuntos de janelas de consulta nas localizações de fornecedores.

No teste foram utilizadas uma base fixa gerada a partir do esquema SpatialDrillAcross com $S F=10$ e $S S F=10$. Nas localizações dos fornecedores foram utilizados quatro diferentes conjuntos de janelas de consulta com seletividade crescente, $Q W_{S 1}, Q W_{S 2}, Q W_{S 3}$ e $Q W_{S 4}$. Já nas localizações dos clientes foi utilizado um conjunto fixo, $Q W_{C 1}$.

Os resultados desse teste de desempenho são exibidos na Figura 5.9. Os gráficos mostram que a estratégia Divide obteve sempre o melhor desempenho, ou seja, um tempo de execução menor em todas as consultas. Nos gráficos também é possível observar que as duas estratégias possuem comportamentos semelhantes conforme a seletividade das consultas é variada. $\mathrm{O}$ acréscimo no tempo de execução em ambas as estratégias é explicado pelo fato de que mais vetores Bitmap são lidos e processados conforme a seletividade aumenta, acarretando também em uma maior quantidade de dados a serem retornados. Tomando como referência o tempo gasto pela estratégia Única, a estratégia Divide atinge uma redução no tempo de 6,5\% a 43,5\%. É importante observar que, apesar da estratégia Divide ter obtido o melhor desempenho, ambas estratégias apresentaram tempos muito positivos no processamento das consultas, e em alguns casos, tempos muito próximos (e.g, consulta Q2.1).

\subsubsection{Divide e Única: Variando o Fator de Escala Convencional}

O objetivo desse teste foi comparar o desempenho entre as estratégias Divide e Única quando o fator de escala convencional (parâmetro $S F$ ) é variado. Com a variação desse parâmetro, é possível verificar como as estratégias se comportam na presença de um volume crescente de 
dados convencionais em todas as consultas, e de dados espaciais para consultas relacionadas às granularidades (s_address_geo) e (c_address_geo).

No teste foram utilizadas seis bases geradas a partir do esquema SpatialDrillAcross. O parâmetro $S S F$ foi mantido fixo com um valor igual a 10. O parâmetro $S F$ foi variado com o objetivo de aumentar o volume de dados convencionais, sendo utilizados os seguintes valores: 5, 10, 15, 20, 25 e 30. Nas localizações dos fornecedores foi utilizado o conjunto de janelas de consulta $Q W_{S 1}$ e nas dos clientes, $Q W_{C 1}$. O tempo de cada consulta corresponde à média de 5 execuções.

Os resultados desse teste de desempenho são exibidos na Figura 5.10. Os gráficos mostram novamente que a estratégia Divide obteve sempre o melhor desempenho, ou seja, um tempo de execução menor em todas as consultas. Para valores de $S F<20 \mathrm{em}$ consultas que estão apenas sofrendo um aumento do volume de dados convencionas (Q1.2, Q1.3, Q1.4, Q2.2, Q2.3 e Q2.4), ambas estratégias apresentam um comportamento linear de crescimento conforme o volume de dados aumenta. A partir do valor $S F=20$, há uma mudança no comportamento de crescimento das duas estratégias. Além disso, a partir desse mesmo valor, a diferença de desempenho entre a estratégia Única e a estratégia Divide aumenta. Tomando como referência o tempo gasto pela estratégia Única, a estratégia Divide atinge uma redução no tempo de $19,4 \%$ a $56,4 \%$. Novamente, é importante observar que, apesar da estratégia Divide ter garantido o melhor desempenho, ambas estratégias apresentaram tempos muito positivos no processamento das consultas, e em alguns casos tempos muito próximos (e.g, consultas Q2.1, Q3.1, Q3.2 e Q3.3).

\subsubsection{Divide e Única: Variando o Fator de Escala Espacial}

O objetivo desse teste foi comparar o desempenho entre as estratégias Divide e Única quando o fator de escala espacial (parâmetro $S S F$ ) é variado. Com a variação do parâmetro $S S F$, é possível verificar como as estratégias se comportam na presença de um volume crescente de dados espaciais nas tabelas City, Nation e Region, isto é, exceto para as consultas Q1.1 e Q2.1 que não se relacionam com essas tabelas.

No teste foram utilizadas quatro bases geradas a partir do esquema SpatialDrillAcross. O valor de $S F=10$ foi mantido, enquanto que o parâmetro $S S F$ foi variado de 5 a 20 , em passos de 5. Nas localizações dos fornecedores foi utilizado o conjunto de janelas de consulta $Q W_{S 1}$ e nas dos clientes, $Q W_{C 1}$.

Os resultados desse teste de desempenho são exibidos na Figura 5.11. Confirmando os resultados anteriores, os gráficos mostram que a estratégia Divide obteve sempre o melhor desempenho, ou seja, um tempo de execução menor em todas as consultas. Nos gráficos é possível observar que as duas estratégias possuem um comportamento bem semelhante conforme o parâmetro $S S F$ é variado. Também é possível observar que o aumento do volume de dados espaciais nas tabelas City, Nation e Region quase não afetou ambas estratégias, que 
apresentaram um comportamento praticamente constante no tempo de execução das consultas. Isso pode ser explicado pelo fato de que as fases de filtragem e refinamento, relacionadas ao processamento do predicado espacial, representam a menor parcela do custo total de cada consulta. A parcela mais custosa está relacionada à resolução da consulta transformada pelo índice Bitmap de junção. Com a manutenção do parâmetro $S F$ e da seletividade da consulta em valores fixos, o custo relacionado a essa última etapa do processamento tornou-se quase constante, definindo o comportamento das curvas. Tomando como referência o tempo gasto pela estratégia Única, a estratégia Divide atinge uma redução no tempo de $19,1 \%$ a 45,6\%. Novamente, é importante observar que, apesar da estratégia Divide ter obtido o meIhor desempenho, ambas estratégias apresentaram tempos muito positivos no processamento das consultas. 


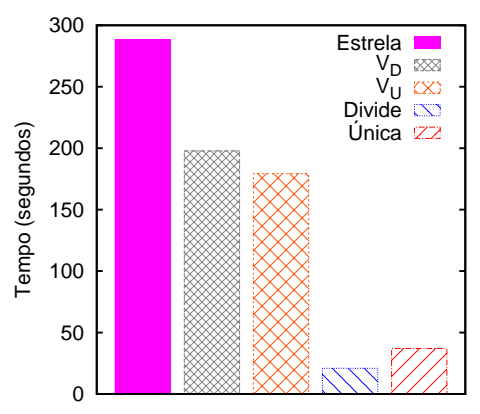

(a) Consulta Q1.1.

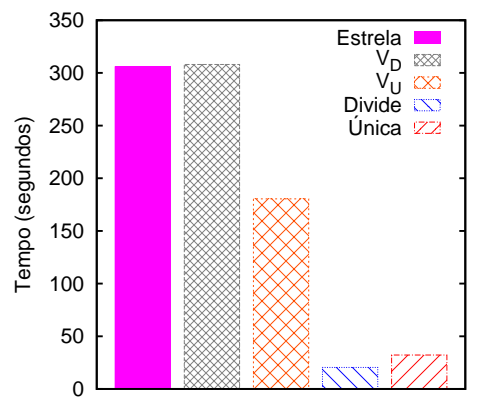

(b) Consulta Q1.2.

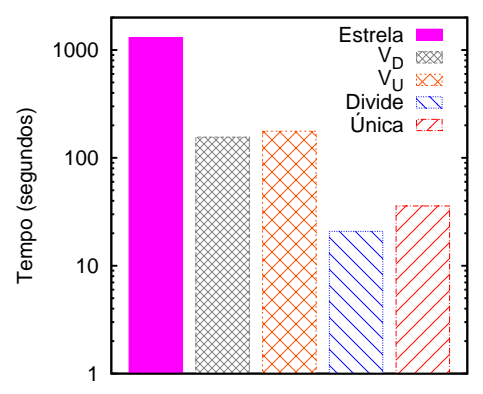

(c) Consulta Q1.3.

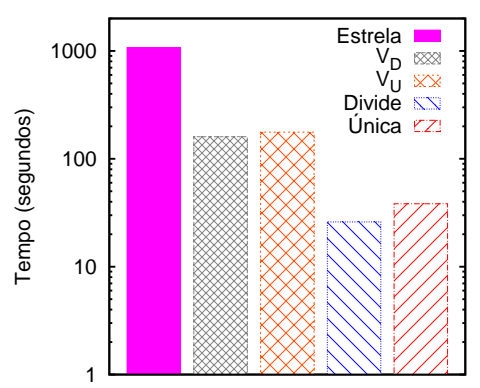

(d) Consulta Q1.4.

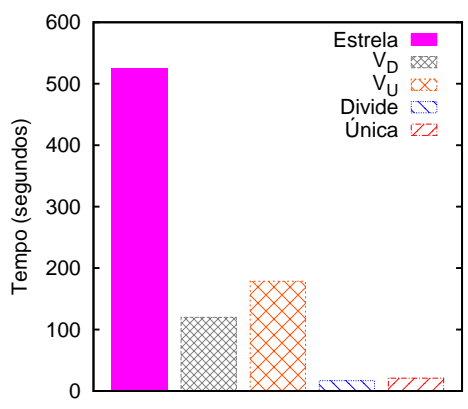

(e) Consulta Q2.1.

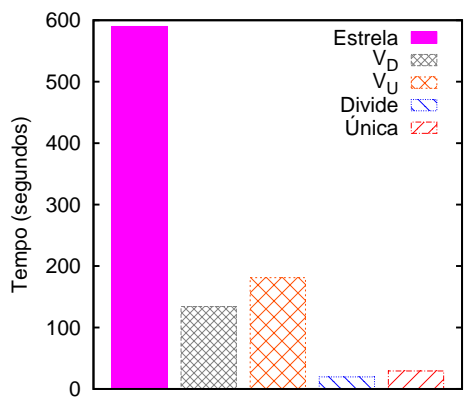

(f) Consulta Q2.2.

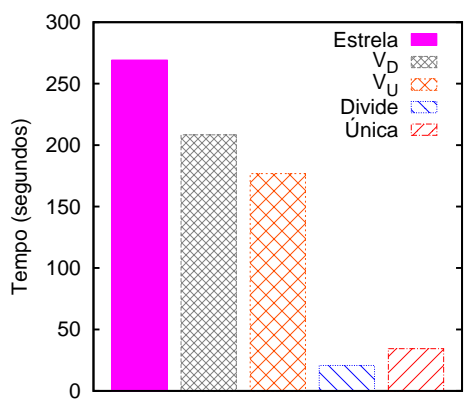

(g) Consulta Q2.3.

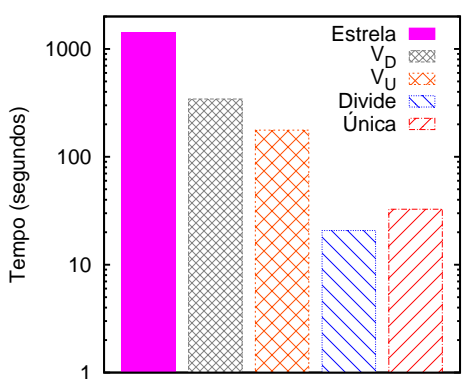

(h) Consulta Q2.4.

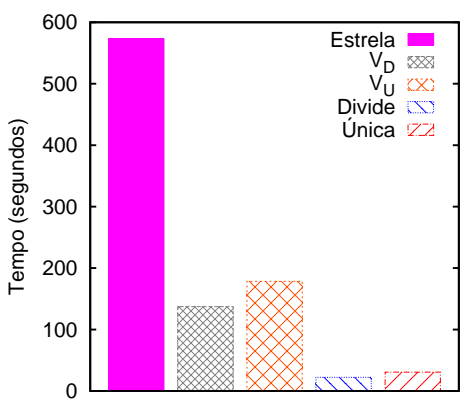

(i) Consulta Q3.1.

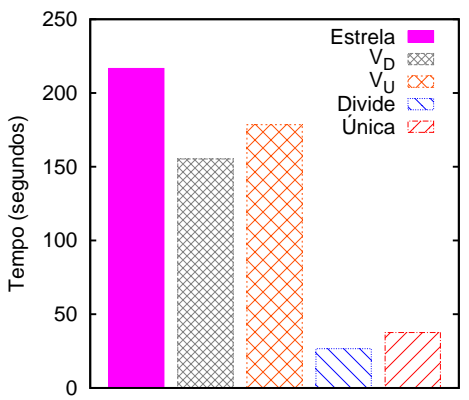

(j) Consulta Q3.2.

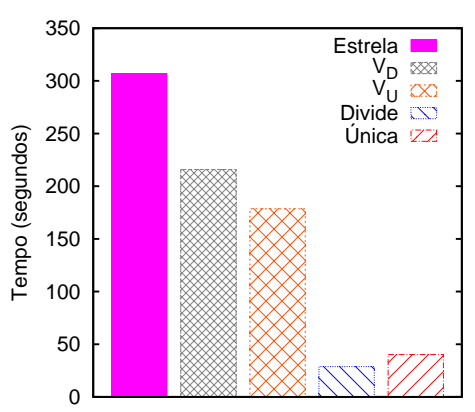

(k) Consulta Q3.3.

Figura 5.8: Comparação do desempenho entre junção estrela, visões materializadas $\bigvee_{D}$ e $\bigvee_{U}$ e as estratégias Divide e Única. O eixo y correspondente ao tempo de execução está em escala logarítmica nas consultas Q1.3, Q1.4 e Q2.4. 


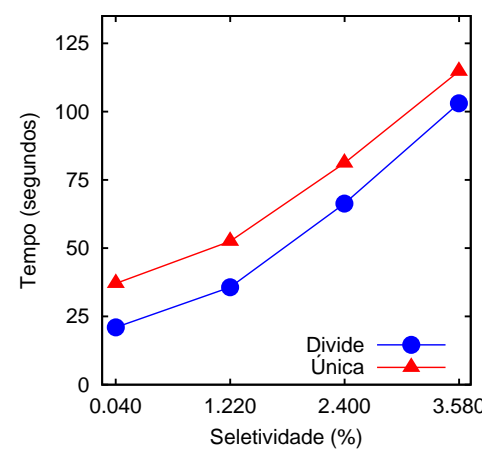

(a) Consulta Q1.1.

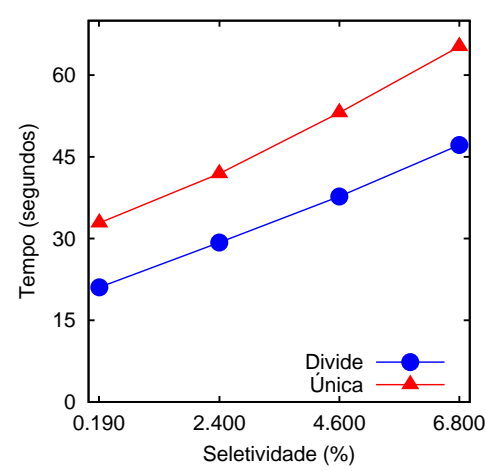

(b) Consulta Q1.2.

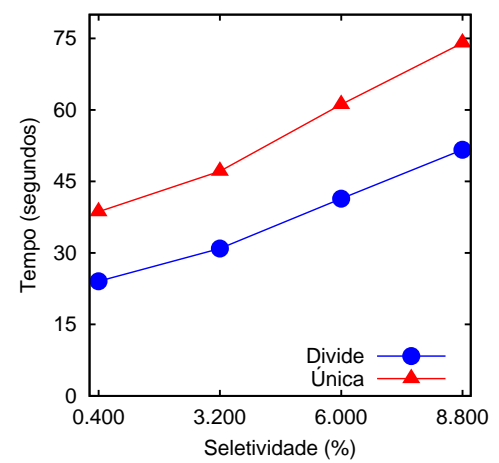

(c) Consulta Q1.3.

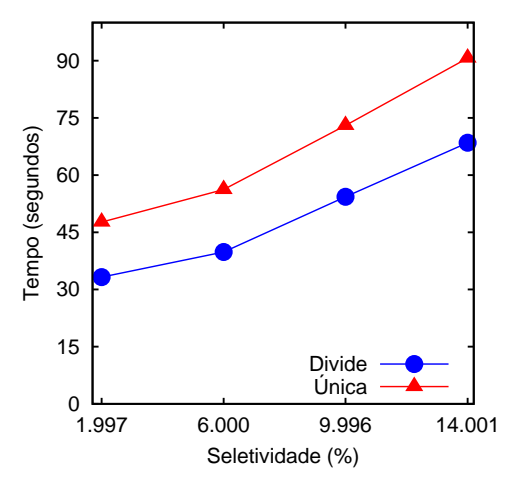

(d) Consulta Q1.4.

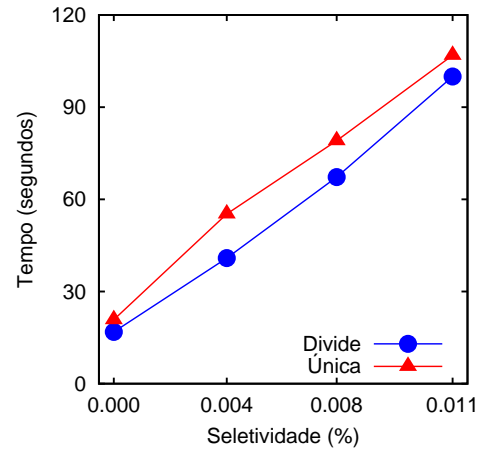

(e) Consulta Q2.1.

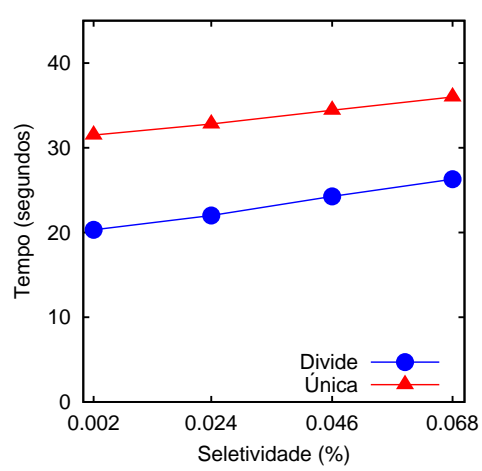

(f) Consulta Q2.2.

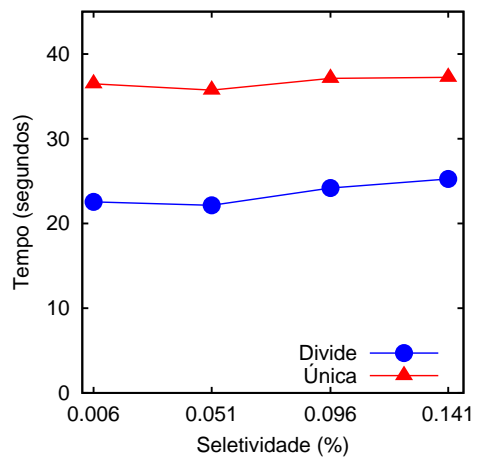

(g) Consulta Q2.3.

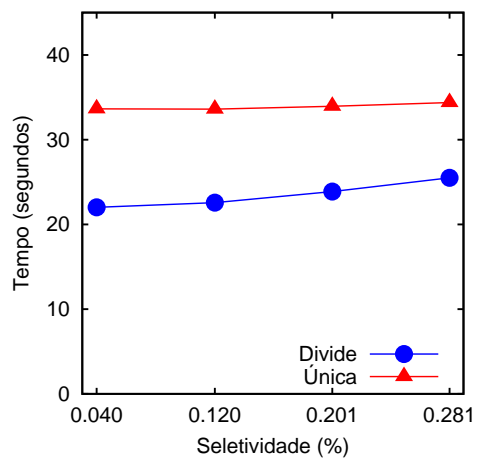

(h) Consulta Q2.4.

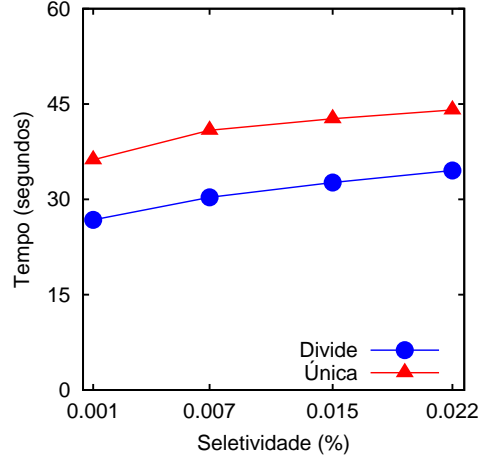

(i) Consulta Q3.1.

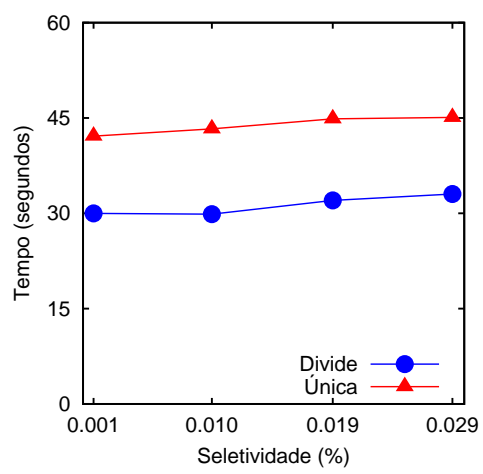

(j) Consulta Q3.2.

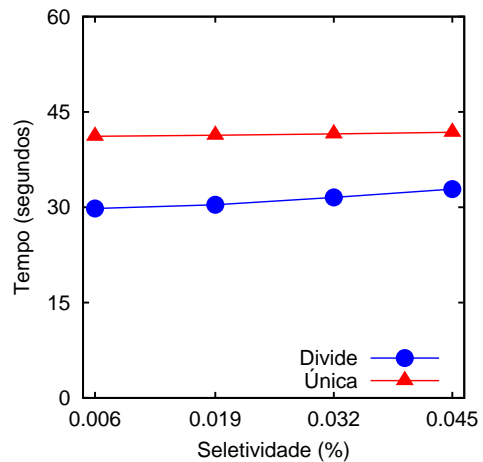

(k) Consulta Q3.3.

Figura 5.9: Desempenho das estratégias Divide e Única variando a seletividade das consultas. 


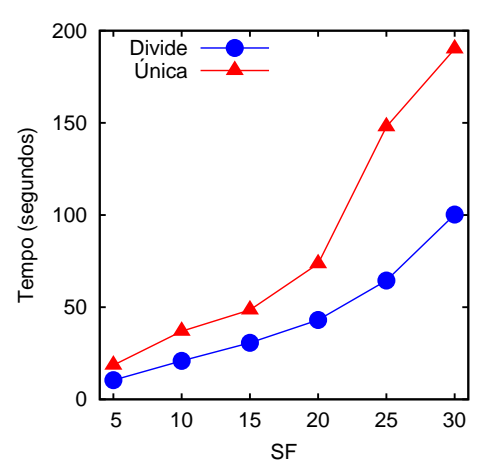

(a) Consulta Q1.1.

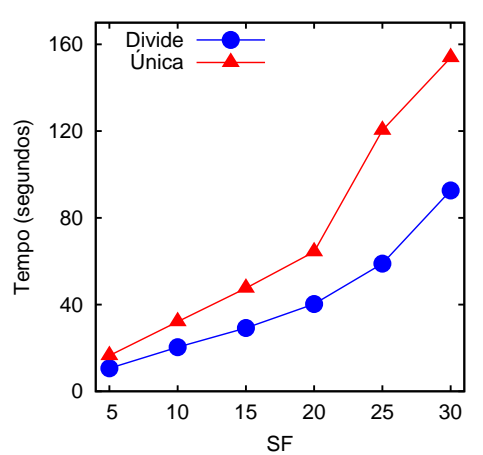

(b) Consulta Q1.2.

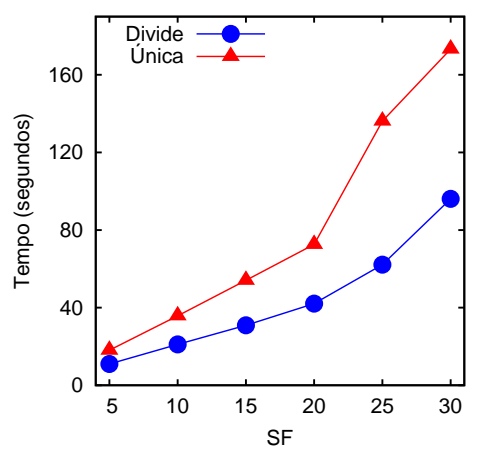

(c) Consulta Q1.3.

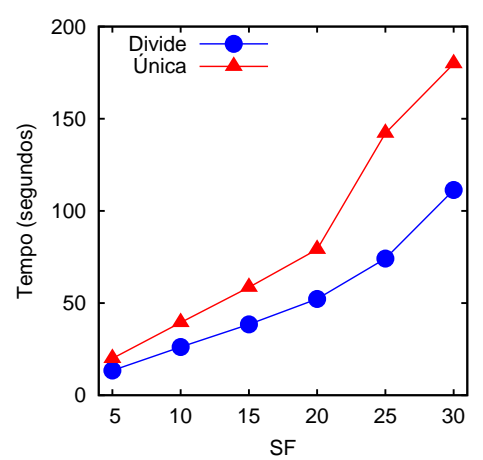

(d) Consulta Q1.4.

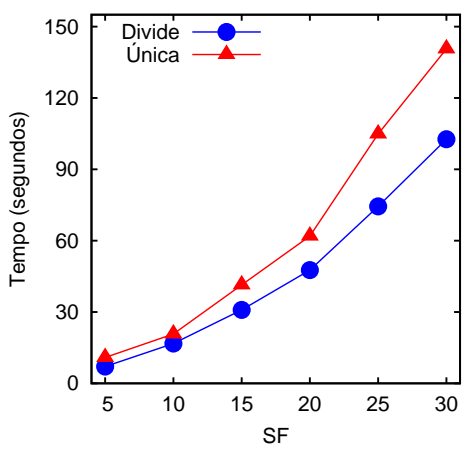

(e) Consulta Q2.1.

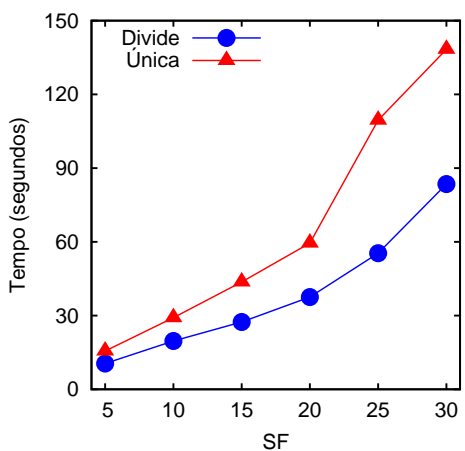

(f) Consulta Q2.2.

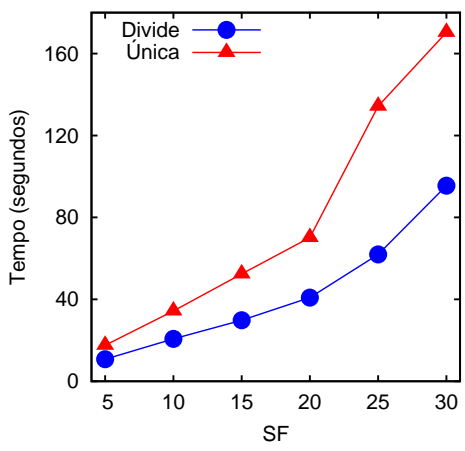

(g) Consulta Q2.3.

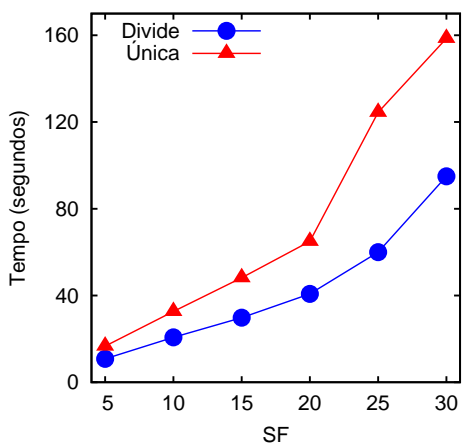

(h) Consulta Q2.4.

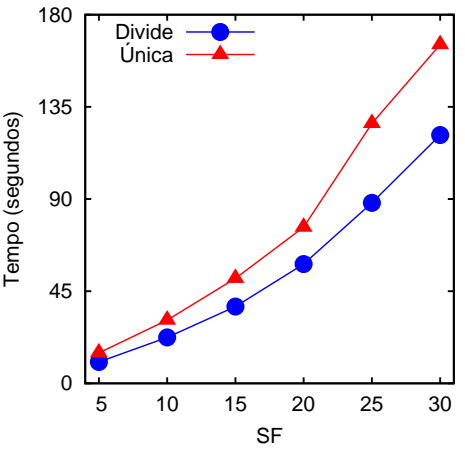

(i) Consulta Q3.1.

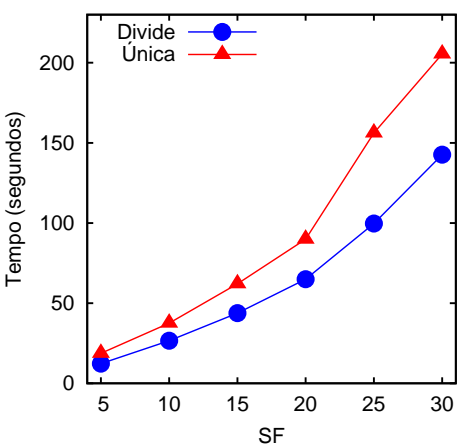

(j) Consulta Q3.2.

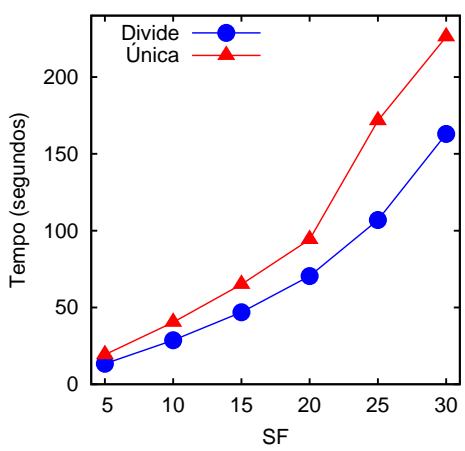

(k) Consulta Q3.3.

Figura 5.10: Desempenho das estratégias Divide e Única variando o fator de escala (SF) convencional. 


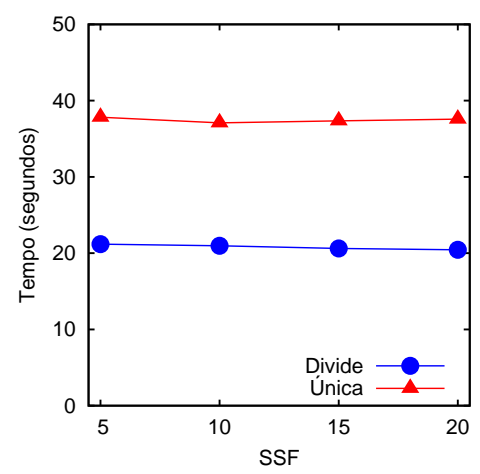

(a) Consulta Q1.1.

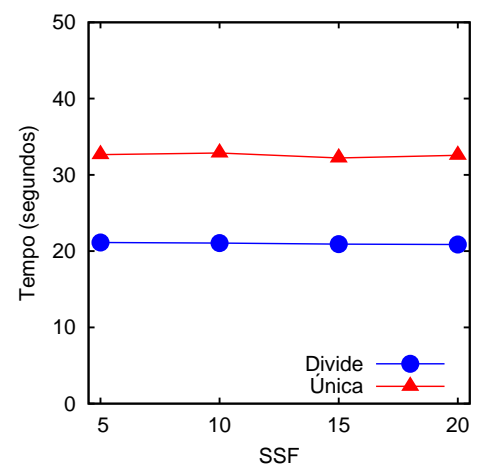

(b) Consulta Q1.2.

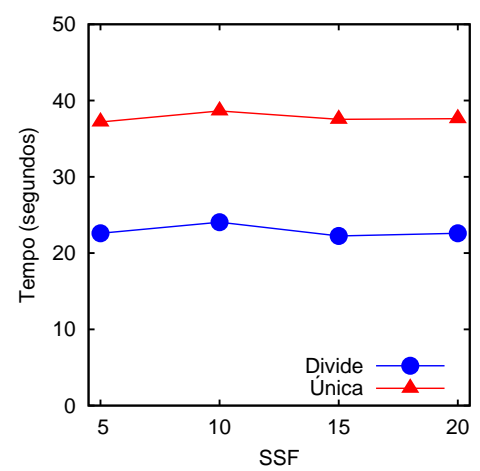

(c) Consulta Q1.3.

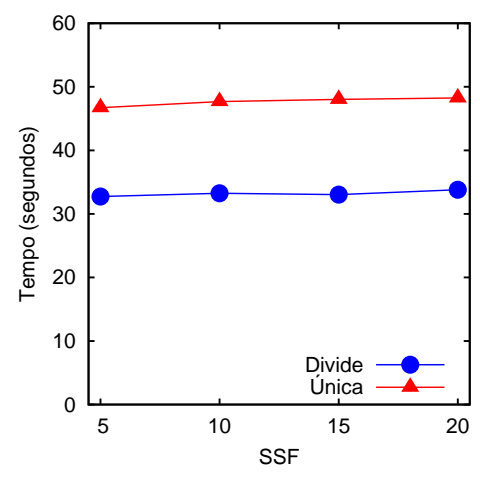

(d) Consulta Q1.4.

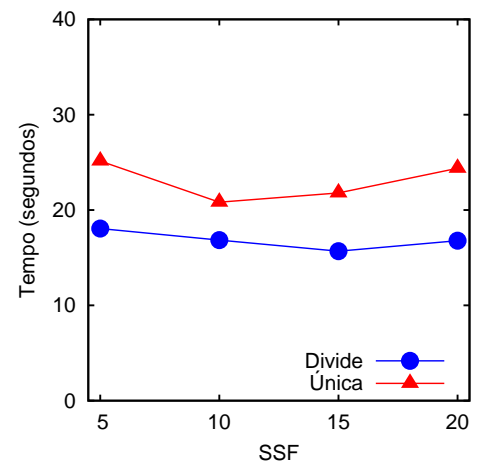

(e) Consulta Q2.1.

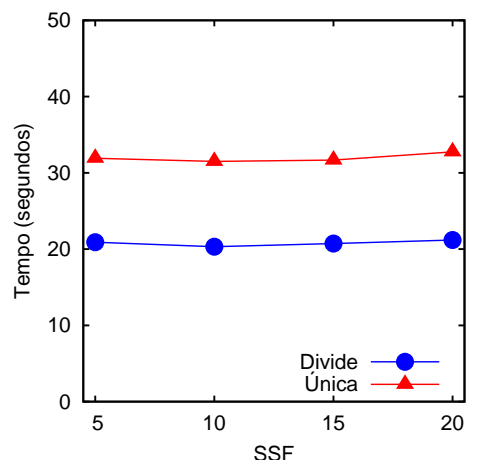

(f) Consulta Q2.2.

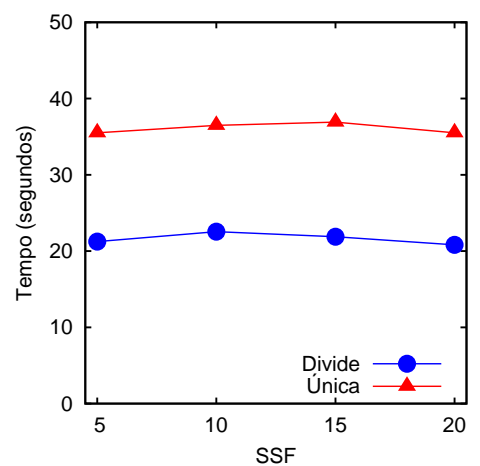

(g) Consulta Q2.3.

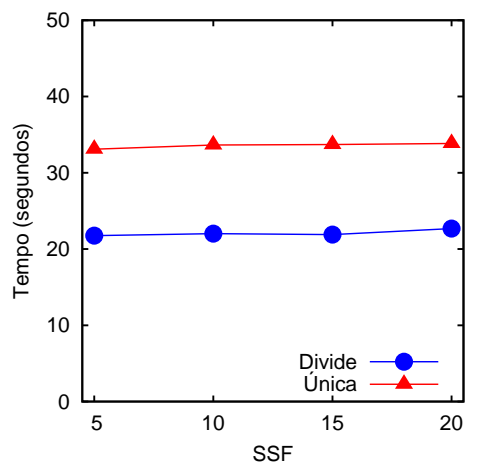

(h) Consulta Q2.4.

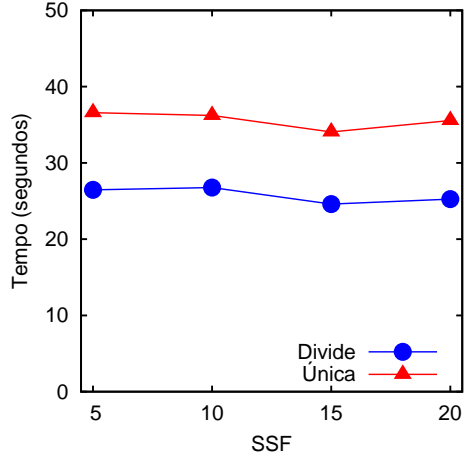

(i) Consulta Q3.1.

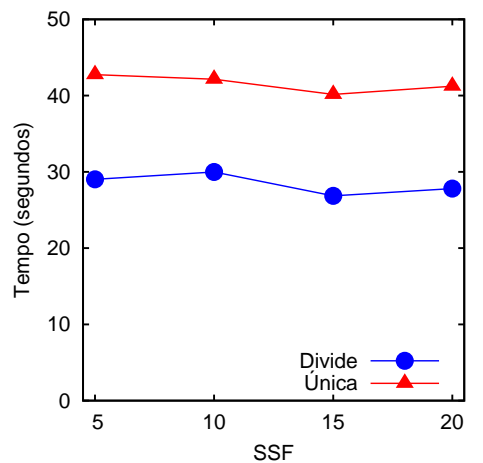

(j) Consulta Q3.2.

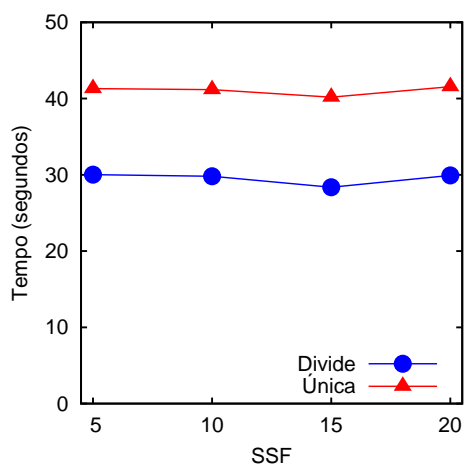

(k) Consulta Q3.3.

Figura 5.11: Desempenho das estratégias Divide e Única variando o fator de escala espacial (SSF). 


\subsubsection{Divide e Única: Variando a Proporção entre Tabelas de Fatos}

O objetivo desse teste foi comparar o desempenho entre as estratégias Divide e Única quando a proporção do número de tuplas nas tabelas de fatos varia. Nos resultados mostrados anteriormente, verificou-se que, apesar da estratégia Divide ter apresentado um desempenho sempre superior à estratégia Única, muitas vezes a diferença de tempos entre ambas estratégias foi bem pequena. Esse fato motivou a investigação de diferentes cenários em que a estratégia Única poderia ser mais indicada ao processamento de consultas SOLAP drill-across.

Analisando o esquema SpatialDrillAcross utilizado nos testes de desempenho mostrados anteriormente, é possível perceber uma característica marcante nesse esquema e que está diretamente ligada às consultas drill-across: a proporção do número de tuplas entre as tabelas de fatos Partsupp e Lineitem. Para esse esquema, essa proporção é igual a 1:7,5, o que significa que a tabela de fatos Lineitem possui 7,5 vezes mais tuplas do que a tabela de fatos Partsupp. Dessa forma, enquanto a estratégia Única constrói um único índice Bitmap de junção com um tamanho proporcional à tabela de fatos Lineitem, a estratégia Divide constrói dois índices Bitmap de junção, um com tamanho proporcional à tabela de fatos Partsupp e outro com tamanho proporcional à tabela de fatos Lineitem. Portanto, a estratégia Única sempre realiza consultas em vetores Bitmap de tamanho $N$, enquanto que a estratégia Divide realiza uma parte da consulta em vetores Bitmap de tamanho $N$ e a outra parte em vetores de tamanho $N / 7,5$. Logo, torna-se interessante investigar como essa proporção afeta o desempenho das estratégias propostas.

Nos testes de desempenho discutidos nessa seção, foram utilizadas cinco bases geradas a partir do esquema SpatialDrillAcross modificado. Todas as bases foram geradas com a configuração $S F=10$ e $S S F=10$, variando apenas o parâmetro $n$ de 1 a 5 , o qual controla a proporção entre as tabelas de fatos Partsupp e Lineitem. Por exemplo, se o valor de $n$ é igual 5, significa que cada tupla da tabela de fatos Partsupp faz junção com 5 tuplas da tabela de fatos Lineitem, e que cada tupla da tabela de fatos Lineitem faz junção com apenas uma tupla da tabela de fatos Partsupp. Quando o parâmetro $n$ é variado e os parâmetros $S F$ e $S S F$ são mantidos fixos, apenas o número de tuplas da tabela de fatos Lineitem varia. O conjunto de janelas de consulta $Q W_{S 1}$ foi utilizado nas localizações dos fornecedores e o conjunto $Q W_{C 1}$ nas localizações dos clientes. Para fins de melhor visualização dos resultados, nos resultados obtidos nesses testes são ilustrados nos gráficos a razão entre o tempo gasto pela estratégia Única $\left(T_{U}\right)$ e o tempo gasto pela estratégia Divide $\left(T_{D}\right)$. Dessa forma, um valor da razão $T_{U} / T_{D}$ abaixo do valor 1 indica que a estratégia Única é mais eficiente.

Os resultados desse teste de desempenho são exibidos na Figura 5.12, onde cada gráfico corresponde a uma classe de consulta. Os gráficos mostram que a estratégia Única obteve o melhor desempenho em todas as consultas quando a proporção entre as tabelas de fatos é igual a 1:1. Tomando como referência o tempo gasto pela estratégia Divide, na proporção 1:1 
a estratégia Única atinge uma redução no tempo de $8,2 \%$ a $41,9 \%$. Apenas para a consulta Q2.1 na proporção 1:2, a estratégia Única ainda é a mais indicada. Conforme a proporção entre as tabelas de fatos aumenta, o valor da razão $T_{U} / T_{D}$ também aumenta, mostrando que a estratégia Única é mais afetada por essa variação.

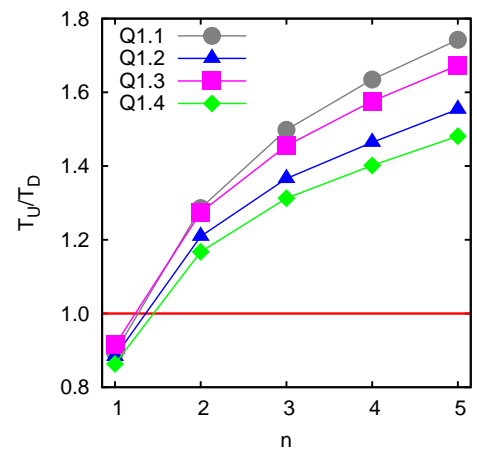

(a) Classe Q1.

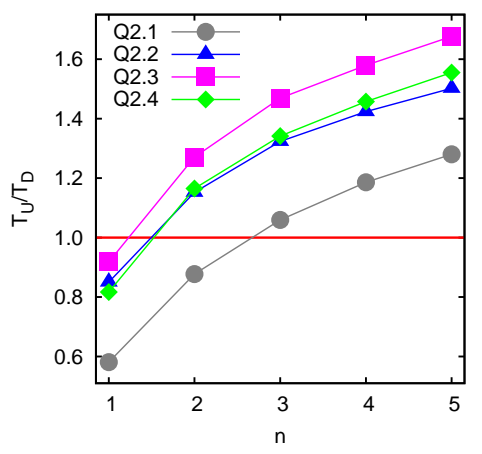

(b) Classe Q2.

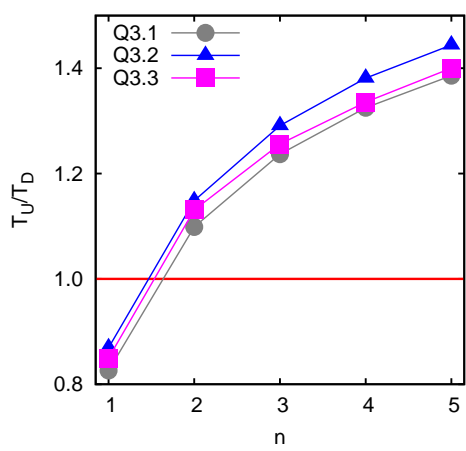

(c) Classe Q3.

Figura 5.12: Razão $T_{U} / T_{D}$ : variação da proporção entre as tabelas de fatos Partsupp e Lineitem.

Nas próximas seções, é investigado como os fatores seletividade das consultas, o fator de escala convencional e o fator de escala espacial afetam o desempenho das estratégias propostas frente à proporção de dados mantidos nas tabelas de fatos. Os resultados ilustrados referem-se a testes realizados até a proporção 1:3, uma vez que a partir desse valor a estratégia Única não é mais recomendada.

\subsubsection{Divide e Única: Variando a Proporção entre Tabelas de Fatos e a Seletividade das Consultas}

O objetivo desse teste foi comparar o desempenho entre as estratégias Divide e Única quando a seletividade das consultas varia, considerando diferentes proporções entre as tabelas de fatos Partsupp e Lineitem. A variação da seletividade é realizada por meio da utilização de diferentes conjuntos de janelas de consulta nas localizações de fornecedores.

No teste foram utilizadas três bases geradas a partir do esquema SpatialDrillAcross modificado. Todas as bases foram geradas com a configuração $S F=10$ e $S S F=10$, variando apenas o parâmetro $n$ de 1 a 3. Nas localizações dos fornecedores foram utilizados quatro conjuntos de janelas de consulta, $Q W_{S 1}, Q W_{S 2}, Q W_{S 3}$ e $Q W_{S 4}$, enquanto que nas localizações dos clientes foi utilizado o conjunto $Q W_{C 1}$.

Os resultados desse teste de desempenho são exibidos na Figura 5.13. Novamente, os gráficos mostram que a estratégia Única obteve o melhor desempenho em todas as consultas quando a proporção entre as tabelas de fatos é igual a 1:1. Além disso, o aumento da seletividade das consultas causou um maior impacto no desempenho da estratégia Divide. Isso acontece porque a estratégia Divide processa duas vezes o predicado espacial relacionado aos 
fornecedores, que é justamente o predicado que está sendo utilizado para variar a seletividade das consultas. Comparando os resultados ilustrados nessa seção com os resultados descritos na seção 5.2.3, pode-se concluir que, conforme a proporção entre as tabelas de fatos aumenta, esse impacto negativo na estratégia Divide é suprimido pelo impacto positivo que o aumento da proporção causa. Portanto, a proporção entre as tabelas de fatos parece ser um fator mais relevante na escolha da estratégia de processamento a ser aplicada. Tomando como referência o tempo gasto pela estratégia Divide, na proporção $1: 1$ a estratégia Única atinge uma redução no tempo de $8,2 \%$ a $48,7 \%$. Confirmando o resultado anterior, apenas para a consulta Q2.1 na proporção 1:2, a estratégia Única ainda é a mais indicada. Conforme a proporção entre as tabelas de fatos aumenta, o valor da razão $T_{U} / T_{D}$ também aumenta, mostrando que a estratégia Única é mais afetada por essa variação.

\subsubsection{Divide e Única: Variando a Proporção entre Tabelas de Fatos e o Fator de Escala Convencional}

O objetivo deste teste foi comparar o desempenho entre as estratégias Divide e Única quando o fator de escala convencional (parâmetro $S F$ ) é variado. Com a variação desse parâmetro, pode-se verificar como as estratégias se comportam na presença de um volume crescente de dados convencionais em todas as consultas e de dados espaciais para consultas relacionadas às granularidades (s_address_geo) e (c_address_geo).

No teste foram utilizadas dezoito bases geradas a partir do esquema SpatialDrillAcross modificado. Todas as bases foram geradas com a seguinte configuração: $S S F=10$, parâmetro $S F$ variando de 5 a 30 (em passos iguais a 5), e parâmetro $n$ com valores iguais a 1,2 e 3 . Nas localizações dos fornecedores foi utilizado o conjunto de janelas de consulta $Q W_{S 1}$, e nas dos clientes, $Q W_{C 1}$. Novamente, é exibida nos gráficos a razão $T_{U} / T_{D}$.

Os resultados desse teste de desempenho são ilustrados na Figura 5.14. Novamente, os gráficos mostram que a estratégia Única obteve o melhor desempenho em todas as consultas quando a proporção entre as tabelas de fatos é igual a 1:1. Também confirmando resultados anteriores, para a consulta Q2.1 e proporção igual a 1:2, a estratégia Única obteve o melhor desempenho. Comparando os resultados mostrados nessa seção e os resultados descritos na seção 5.2.4, pode-se observar a mesma mudança de comportamento das estratégias para $S F=20$, embora dessa vez essa mudança seja mais suave para as proporções consideradas. Isso pode ser considerado um indicativo de que o aumento da proporção entre as tabelas de fatos acentua essa mudança de comportamento. Além disso, para o valor $S F=30$, a razão $T_{U} / T_{D}$ se aproxima bastante do valor $1 \mathrm{em}$ algumas consultas (Q1.1, Q1.2, Q1.3 e Q2.3), indicando que mesmo para a proporção $1: 1$, a estratégia Única pode deixar de ser a melhor opção para valores de $S F>30$. Portanto, além da proporção entre as tabelas de fatos, o volume de dados demonstrou ser um fator relevante na escolha da estratégia de processamento a ser aplicada. Tomando como referência o tempo gasto pela estratégia Divide, 
na proporção 1:1 a estratégia Única praticamente empata com a estratégia Divide (consulta Q1.3 e $S F=30)$ e atinge uma redução no tempo máxima de $41,9 \%$. Novamente, conforme a proporção entre as tabelas de fatos aumenta, o valor da razão $T_{U} / T_{D}$ também aumenta, mostrando que a estratégia Única é mais afetada por essa variação.

\subsubsection{Divide e Única: Variando a Proporção entre Tabelas de Fatos e o Fator de Escala Espacial}

O objetivo desse teste foi comparar o desempenho entre as estratégias Divide e Única quando o fator de escala espacial (parâmetro $S S F$ ) é variado, considerando diferentes proporções entre as tabelas de fatos Partsupp e Lineitem. Com a variação desse parâmetro pode-se verificar como as estratégias se comportam na presença de um volume crescente de dados espaciais nas tabelas City, Nation e Region, isto é, exceto para as consultas Q1.1 e Q2.1 que não se relacionam com essas tabelas.

No teste foram utilizadas doze bases geradas a partir do esquema SpatialDrillAcross modificado. Todas as bases foram geradas com a seguinte configuração: $S F=10$, parâmetro $S S F$ variando de 5 a 20 (em passos iguais a 5), e parâmetro $n$ com valores iguais a 1,2 e 3 . Nas localizações dos fornecedores foi utilizado o conjunto de janelas de consulta $Q W_{S 1}$, e nas dos clientes, $Q W_{C 1}$. Novamente, nesse teste é mostrada nos gráficos a razão $T_{U} / T_{D}$.

Os resultados desse teste de desempenho são exibidos na Figura 5.15. Novamente, os gráficos mostram que a estratégia Única obteve o melhor desempenho em todas as consultas quando a proporção entre as tabelas de fatos é igual a 1:1. Confirmando resultados anteriores, para a consulta Q2.1 e proporção igual a 1:2, a estratégia Única obteve o melhor desempenho para valores $S S F>=10$. Comparando os resultados descritos nessa seção com os resultados exibidos na seção 5.2.5, conclui-se que a proporção entre as tabelas de fatos é o fator mais importante na escolha da estratégia de processamento a ser aplicada, uma vez que a razão $T_{U} / T_{D}$ tende a ser uniforme com a variação de $S S F$. Como explicado anteriormente, esse comportamento se deve ao fato de que as fases de filtragem e refinamento, relacionadas ao processamento do predicado espacial, representam a menor parcela do custo de processamento total de cada consulta. A parcela mais custosa está relacionada com a resolução da consulta transformada pelo índice Bitmap de junção. Com os parâmetros $S F$ e da seletividade da consulta fixos, o custo relacionado a essa última etapa do processamento tornou-se quase constante, definindo o comportamento das curvas. Tomando como referência o tempo gasto pela estratégia Divide, na proporção 1:1 a estratégia Única atinge uma redução no tempo de $9,5 \%$ a $49,2 \%$. Novamente, conforme a proporção entre as tabelas de fatos aumenta, o valor da razão $T_{U} / T_{D}$ também aumenta, mostrando que a estratégia Única é mais afetada por essa variação.

Uma possível explicação para o comportamento anômalo das consultas Q1.1 e Q2.1 no valor $S S F=5$ pode ser feita com base na análise dos tamanhos dos índices Bitmap de 
junção gerados pelo software FastBit empregado pelo SB-index. Quando um conjunto de atributos é indexado pelo software FastBit, todas as opções de otimização (e.g., binning) são aplicadas a todos os atributos. Portanto, não é possível designar opções para atributos específicos. Além disso, por padrão o FastBit comprime os vetores Bitmap gerados. Em todas as bases de dados foram aplicadas as mesmas opções de otimização. Porém, índices de tamanhos significativamente menores foram gerados para o atributo suppkey quando $S S F=$ 5. Na consulta Q1.1, o predicado transformado está diretamente relacionado a esse atributo. Assim, a estratégia Única foi beneficiada pelo fato de processar esse predicado uma única vez, diminuindo significativamente o valor da razão $T_{U} / T_{D}$ nesse ponto. Já para a consulta Q2.1, que possui dois predicados, houve um comportamento inverso no ponto $S S F=5$ : a razão $T_{U} / T_{D}$ aumentou. Esse aumento pode ser explicado devido ao fato de que a estratégia Única processa os dois predicados juntos e uma única vez, o que fez com que o tamanho reduzido do índice Bitmap de junção do atributo suppkey não afetasse seu processamento, tornando praticamente constante o tempo para todos os valores de $S S F$. Já na estratégia Divide, a etapa correspondente à subconsulta da tabela de fatos Partsupp foi significativamente afetada por essa redução no Bitmap desse atributo. Com isso, apenas a estratégia Divide foi beneficiada, enquanto a estratégia Única manteve seu tempo de execução praticamente constante. Consequentemente, a razão $T_{U} / T_{D}$ aumentou nesse ponto. Esses resultados demonstram que as características dos dados são um fator importante a ser considerado na comparação das estratégias propostas caso o FastBit seja utilizado. 


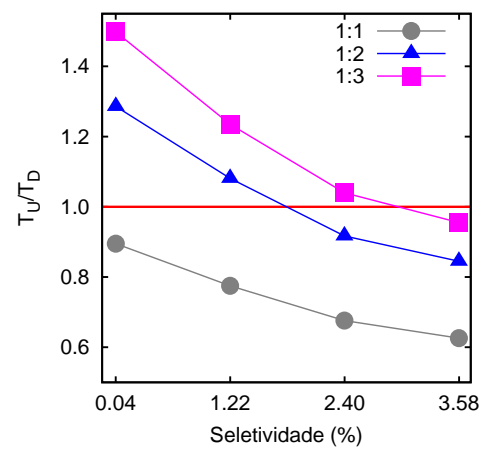

(a) Consulta Q1.1.

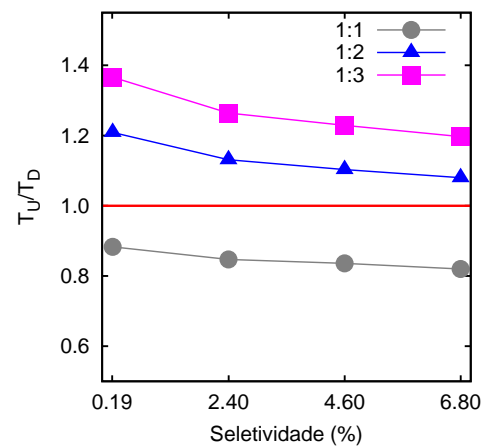

(b) Consulta Q1.2.

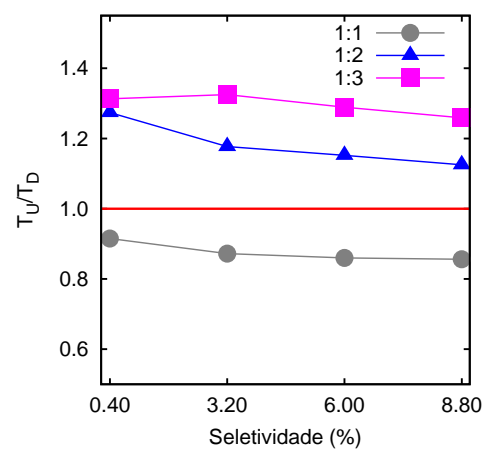

(c) Consulta Q1.3.

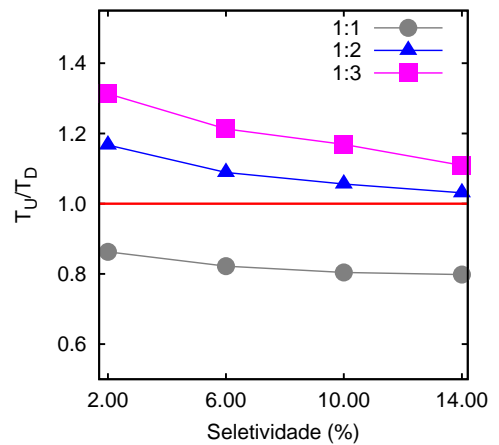

(d) Consulta Q1.4.

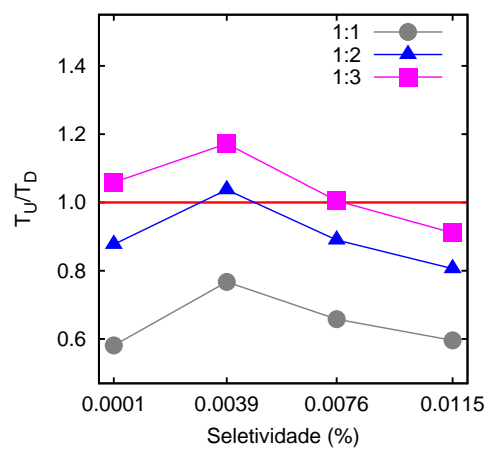

(e) Consulta Q2.1.

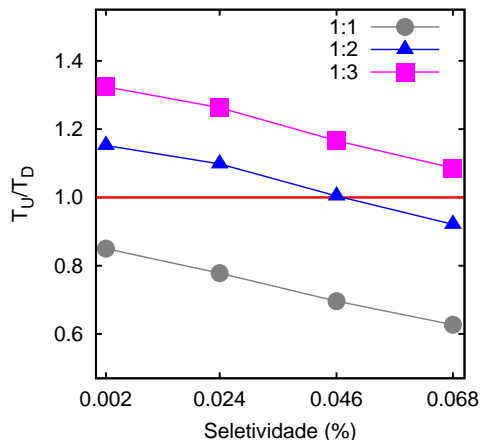

(f) Consulta Q2.2.

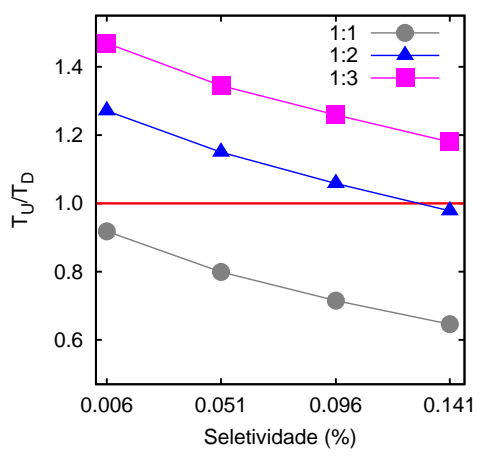

(g) Consulta Q2.3.

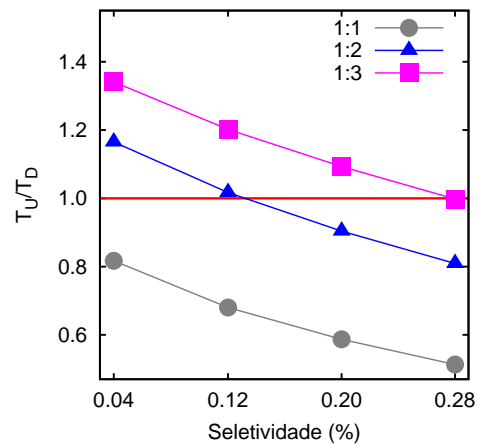

(h) Consulta Q2.4.

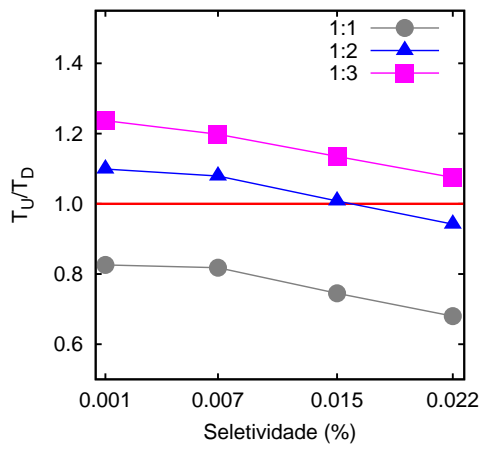

(i) Consulta Q3.1.

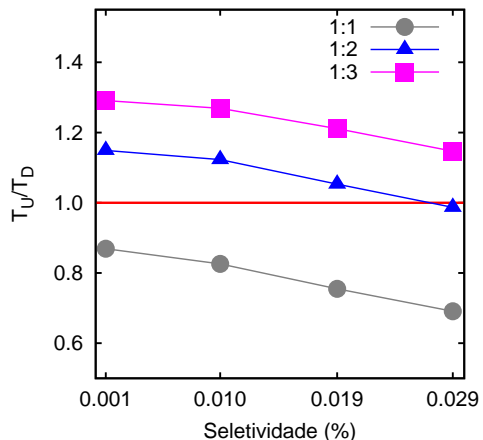

(j) Consulta Q3.2.

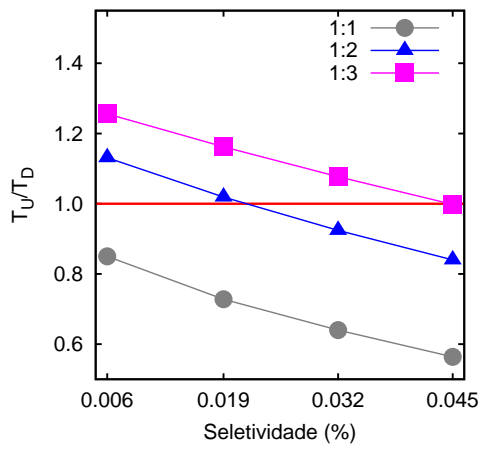

(k) Consulta Q3.3.

Figura 5.13: Razão $T_{U} / T_{D}$ : variação da proporção entre as tabelas de fatos Partsupp e Lineitem e da seletividade das consultas. 


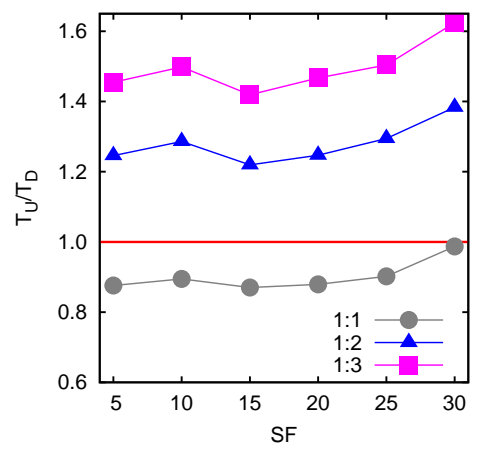

(a) Consulta Q1.1.

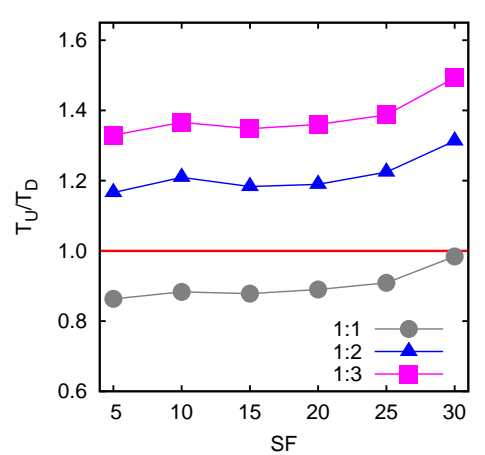

(b) Consulta Q1.2.

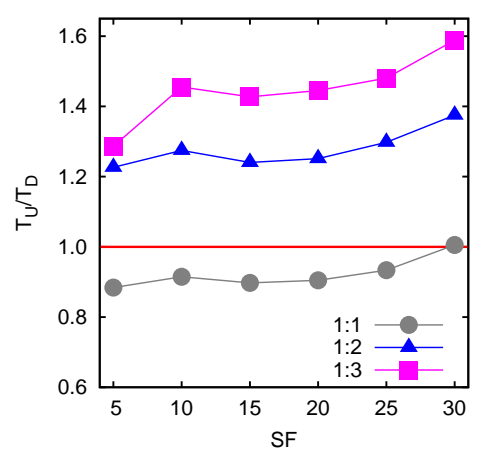

(c) Consulta Q1.3.

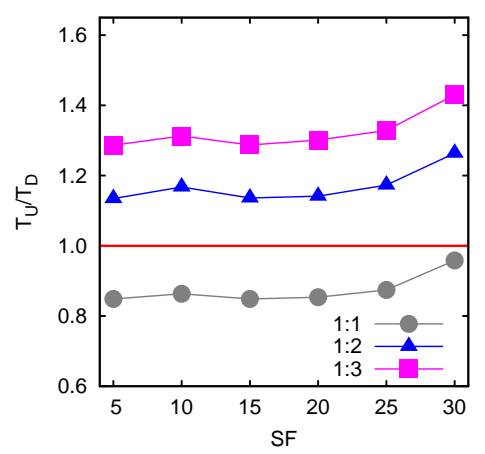

(d) Consulta Q1.4.

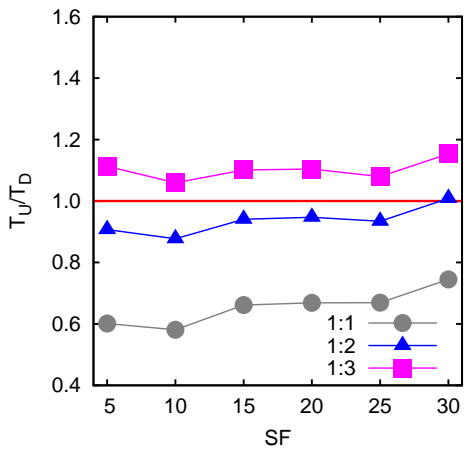

(e) Consulta Q2.1.

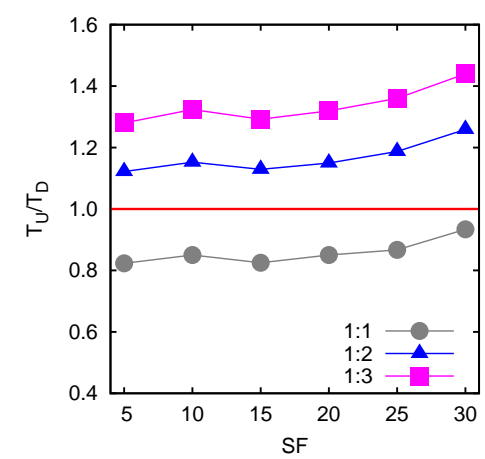

(f) Consulta Q2.2.

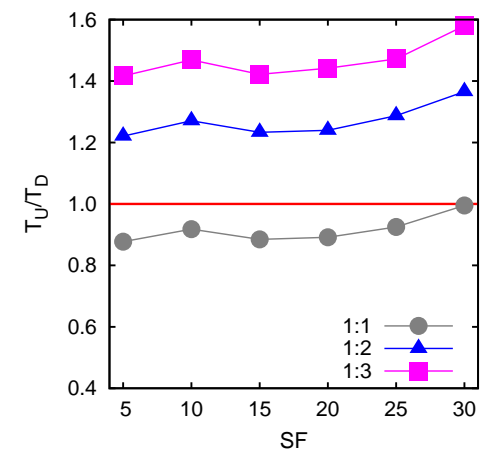

(g) Consulta Q2.3.

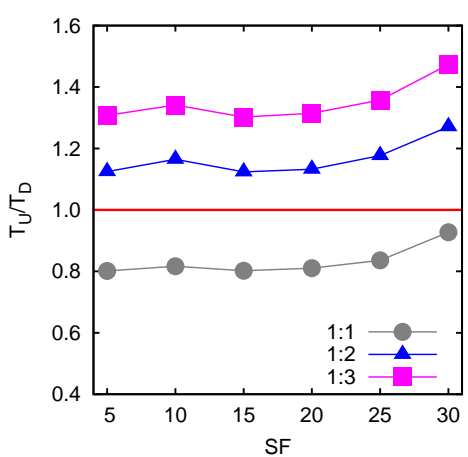

(h) Consulta Q2.4.

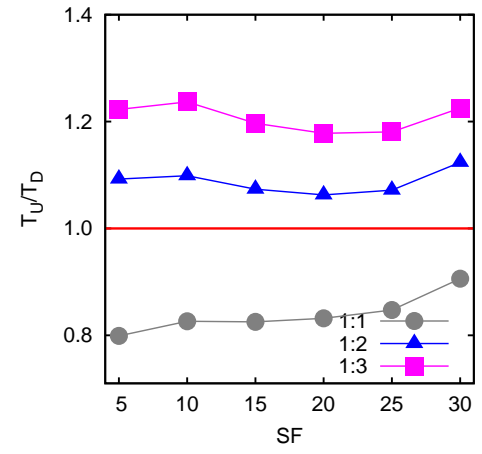

(i) Consulta Q3.1.

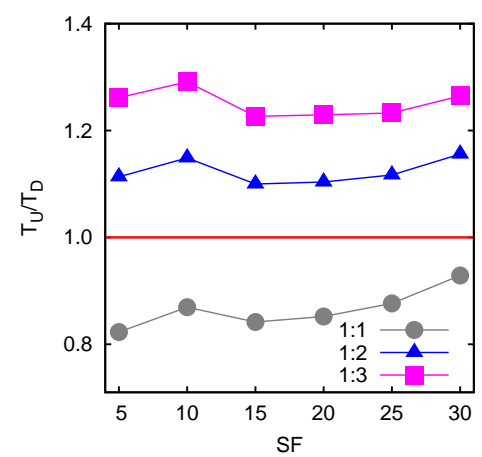

(j) Consulta Q3.2.

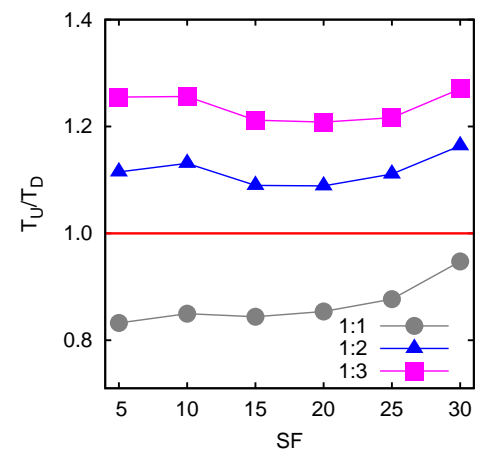

(k) Consulta Q3.3.

Figura 5.14: Razão $\mathrm{T}_{U} / \mathrm{T}_{D}$ : variação da proporção entre as tabelas de fatos Partsupp e Lineitem e do fator de escala (SF) convencional. 


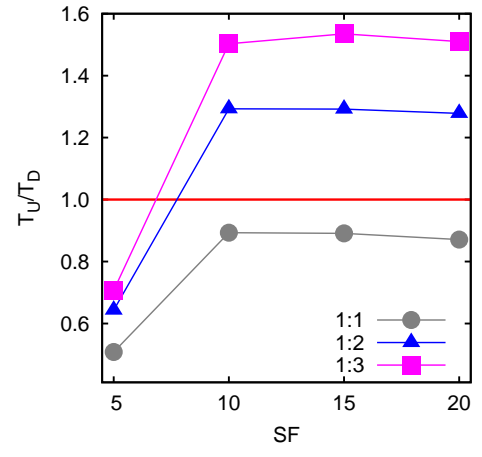

(a) Consulta Q1.1.

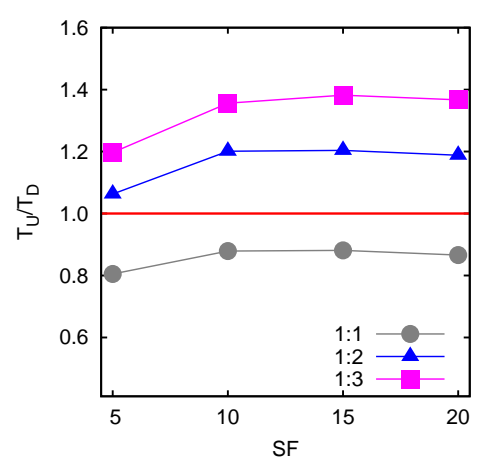

(b) Consulta Q1.2.

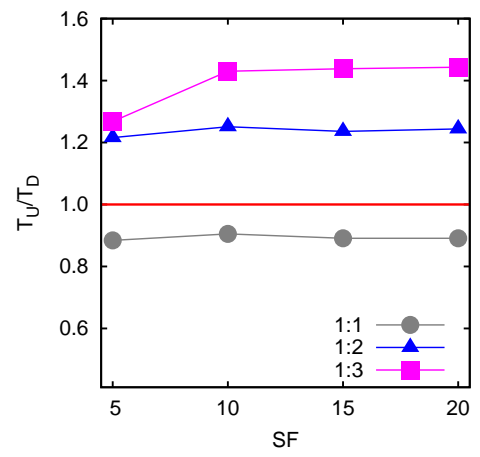

(c) Consulta Q1.3.

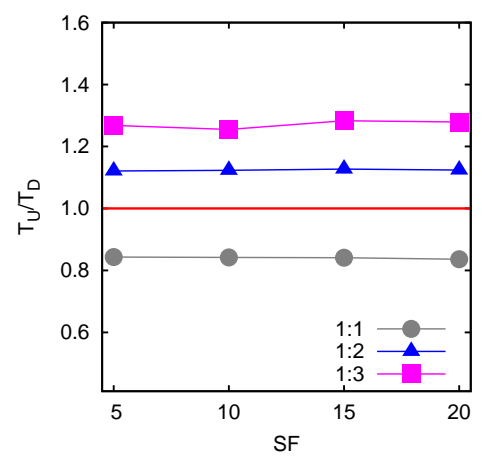

(d) Consulta Q1.4.

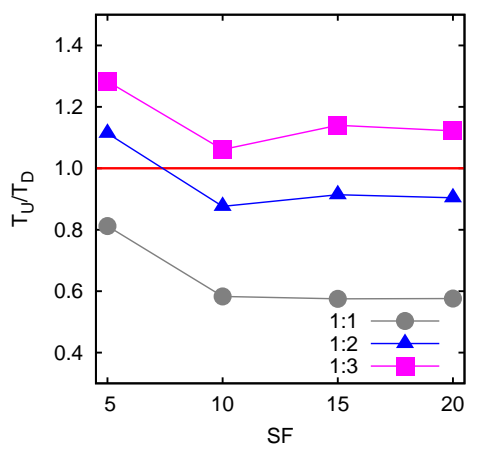

(e) Consulta Q2.1.

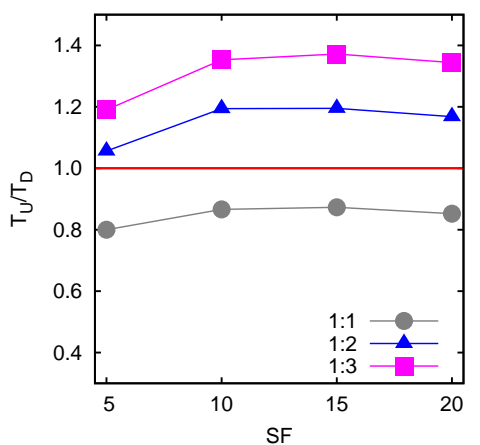

(f) Consulta Q2.2.

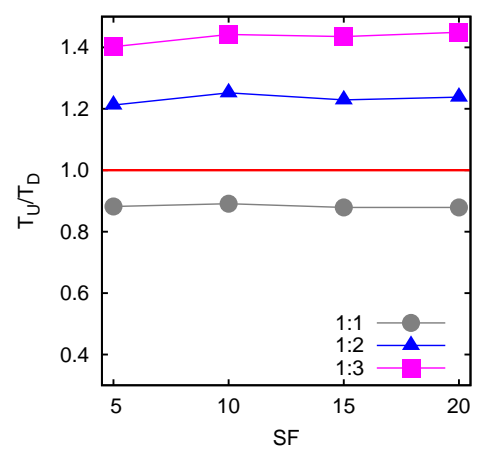

(g) Consulta Q2.3.

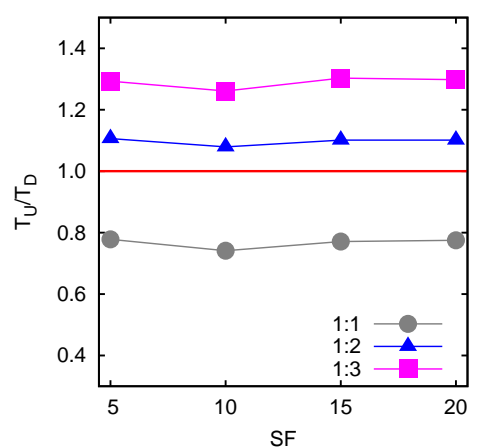

(h) Consulta Q2.4.

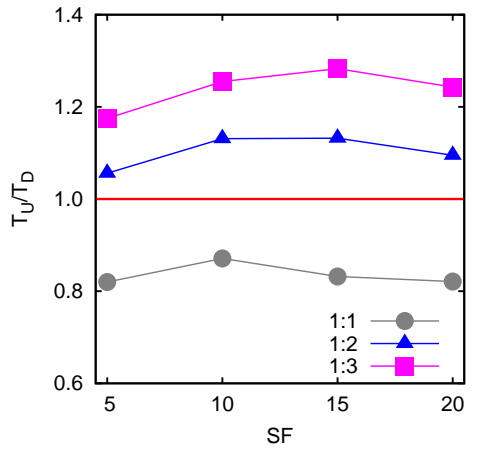

(i) Consulta Q3.1.

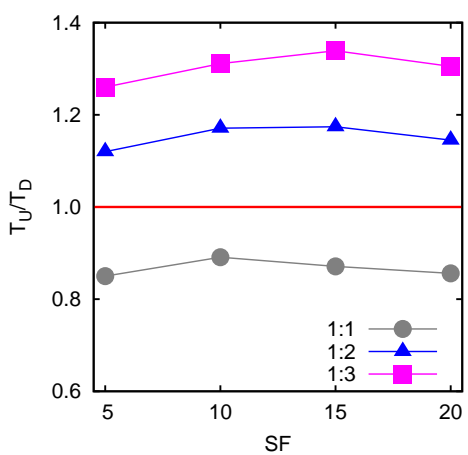

(j) Consulta Q3.2.

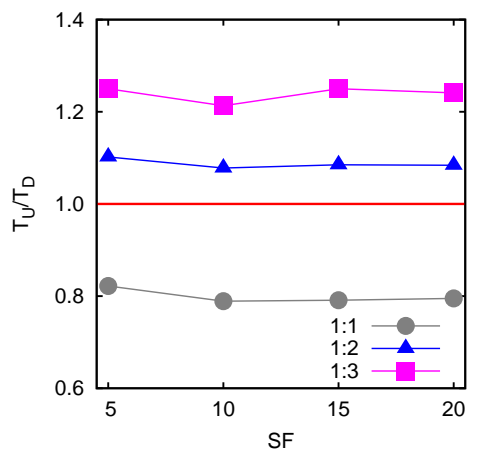

(k) Consulta Q3.3.

Figura 5.15: Razão $T_{U} / T_{D}$ : variação da proporção entre as tabelas de fatos Partsupp e Lineitem e do fator de escala espacial (SSF). 


\subsection{Considerações Finais}

Neste capítulo foram apresentadas duas estratégias de processamento de consultas SOLAP drill-across em DWGs, as quais são baseadas no índice para DWG SB-index. A estratégia Divide baseia-se na indexação individual de esquemas estrela de uma constelação de fatos e na divisão de uma consulta em várias subconsultas para produzir subresultados que são combinados, agrupados e ordenados para obter o resultado final. A estratégia Única baseia-se na indexação unificada da constelação de fatos e na execução completa da consulta sobre esse único índice.

Adicionalmente, visando oferecer fundamentação para as estratégias propostas, foram pesquisados dois aspectos desafiadores relacionados, os quais referem-se: (i) à especificação de um conjunto de diretrizes que deve ser seguido para o projeto de um esquema de DWG que possibilite a execução de consultas SOLAP drill-across, e (ii) à especificação de classes de consultas SOLAP drill-across que introduzem diferentes custos de processamento, de forma que essas consultas sejam executadas sobre um DWG projetado de acordo com as diretrizes propostas.

As estratégias Divide e Única foram contrastadas contra a junção estrela e o uso de visões materializadas. Nos testes de desempenho realizados, as estratégias propostas apresentaram desempenho muito superior aos concorrentes, o qual variou de $82,7 \%$ a $98,6 \%$. Comparando apenas a estratégia Divide com a estratégia Única, os testes de desempenho demonstraram que a estratégia Divide é a mais eficiente na maioria dos cenários. Entretanto, em cenários específicos, nos quais a proporção entre as tabelas de fatos é $1: 1$, a estratégia Única demonstrou ser a melhor opção na grande maioria dos casos.

No próximo capítulo é descrita a proposta de processamento para consultas SOLAP com junção espacial utilizando o SB-index. 


$\mathbf{m}$

\section{Processamento de Consultas SOLAP com Junção Espacial}

Este capítulo tem como objetivo propor uma estratégia de processamento para consultas SOLAP com junção espacial em DWGs. Assim como no processamento de consultas SOLAP drill-across, a nova estratégia proposta é baseada no SB-index e, portanto, também pode ser caracterizada como uma extensão dos algoritmos do SB-index para enfocar esse tipo de consulta SOLAP complexa. Em especial, a principal motivação para a proposta dessa estratégia refere-se ao fato de que nenhum trabalho na literatura é voltado ao processamento eficiente de consultas SOLAP com junção espacial, como descrito no Capítulo 4.

Este capítulo está organizado da seguinte forma. Na seção 6.1 é detalhada a proposta para o processamento de consultas SOLAP com junção espacial. Na seção 6.2 são discutidos os resultados dos testes de desempenho. O capítulo é finalizado na seção 6.3 , com as considerações finais.

\subsection{Proposta}

Visando oferecer fundamentação para a proposta da estratégia de processamento de consultas SOLAP com junção espacial, foram pesquisados dois aspectos relacionados, os quais referemse ao esquema de DWG e à definição de conjuntos de consultas. Portanto, esta dissertação inclui as seguintes contribuições: 
- A identificação de quais características o esquema de DWG deve possuir para possibilitar a execução de consultas SOLAP com junção espacial.

- A especificação do formato de consultas SOLAP com junção espacial, de forma que essas consultas sejam executadas sobre um DWG projetado de acordo com as características identificadas.

- A proposta da estratégia SJB, a qual consiste em uma abordagem baseada no índice SB-index para o processamento de consultas SOLAP com junção espacial.

Essas contribuições são detalhadas nas seções 6.1 .1 a 6.1.3, respectivamente.

\subsubsection{Características do Esquema}

Na seção 5.1.1 foi definido um conjunto de diretrizes que devem ser seguidas para o projeto de um DWG que possibilite a execução de consultas SOLAP drill-across. No caso de consultas SOLAP com junção espacial, o requisito necessário é a existência de duas ou mais dimensões espaciais. Consultas SOLAP são sempre realizadas em pelo menos uma tabela de fatos. Isto significa que pelo menos uma das dimensões espaciais deve ser diretamente ou indiretamente referenciada por uma tabela de fatos presente na consulta.

\subsubsection{Formato de Consultas}

Benchmarks de DWGs existentes na literatura (e.g., Spadawan) não possuem consultas SOLAP com junção espacial. Portanto, nessa seção é definido o formato que consultas SOLAP com junção espacial devem possuir.

Para consultas SOLAP com junção espacial, os predicados espaciais das consultas podem utilizar uma ou duas tabelas de dimensão espacial, isto é, uma ou duas tabelas que são referenciadas de forma direta ou indireta pela tabelas de fatos. Além disso, as consultas são agrupadas em conjuntos. Um conjunto é formado sempre que um percurso é realizado em apenas uma hierarquia espacial. Consultas de um mesmo conjunto que diferenciam-se somente pelo nível de granularidade são organizadas e enumeradas em ordem crescente de granularidade. Todas as consultas possuem uma estrutura semelhante e compartilham os mesmos predicados convencionais. Essa forma de agrupar as consultas visa apenas organizá-las, caracterizando conjuntos de consultas SOLAP roll-up/drill-down com junção espacial. Dois exemplos de formação de conjuntos de consultas são dados a seguir.

\section{Exemplo 4}

Considere duas hierarquias espaciais, $\left(H_{\text {região }}\right) \preceq\left(H_{\text {estado }}\right) \preceq\left(H_{\text {cidade }}\right) \preceq\left(H_{\text {rua }}\right)$ e $\left(P_{\text {região }}\right) \preceq\left(P_{\text {estado }}\right) \preceq\left(P_{\text {cidade }}\right) \preceq\left(P_{\text {rua }}\right)$, relacionadas a duas dimensões espaciais, Hospital e Paciente, envolvidas nos predicados espaciais. Q1 e Q2 são dois possíveis conjuntos formados com essas hierarquias. 
- Q1.1: junção espacial entre $\left(H_{\text {rua }}\right)$ e $\left(P_{\text {rua }}\right)$.

- Q1.2: junção espacial entre $\left(H_{\text {rua }}\right)$ e $\left(P_{\text {cidade }}\right)$.

- Q1.3: junção espacial entre $\left(H_{\text {rua }}\right)$ e $\left(P_{\text {estado }}\right)$.

- Q1.4: junção espacial entre $\left(H_{\text {rua }}\right)$ e $\left(P_{\text {região }}\right)$.

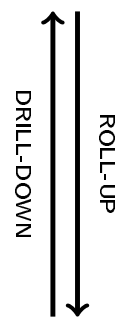

- Q2.1: junção espacial entre $\left(H_{\text {cidade }}\right)$ e $\left(P_{\text {rua }}\right)$.

- Q2.2: junção espacial entre $\left(H_{\text {cidade }}\right)$ e $\left(P_{\text {cidade }}\right)$.

- Q2.3: junção espacial entre $\left(H_{\text {cidade }}\right)$ e $\left(P_{\text {estado }}\right)$.

- Q2.4: junção espacial entre $\left(H_{\text {cidade }}\right)$ e $\left(P_{\text {região }}\right)$.

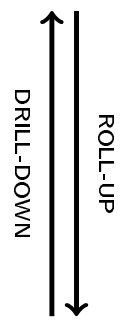

Em cada conjunto, a hierarquia de Hospital permanece em uma granularidade fixa e a hierarquia de Paciente é percorrida.

\section{Exemplo 5}

Considere a mesma hierarquia espacial de Hospital, $\left(H_{\text {região }}\right) \preceq\left(H_{\text {estado }}\right) \preceq$ $\left(H_{\text {cidade }}\right) \preceq\left(H_{\text {rua }}\right)$. Considere também dados espaciais sobre aeroportos, representados por (Aeroporto), que não são referenciados por tabelas de fatos. Q3 representa um possível conjunto formardo pelo percurso da hierarquia de Hospital.

- Q3.1: junção espacial entre (Aeroporto) e $\left(H_{\text {rua }}\right)$.

- Q3.2: junção espacial entre (Aeroporto) e ( $\left.H_{\text {cidade }}\right)$.

- Q3.3: junção espacial entre (Aeroporto) e $\left(H_{\text {estado }}\right)$.

- Q3.4: junção espacial entre (Aeroporto) e $\left(H_{\text {região }}\right)$.

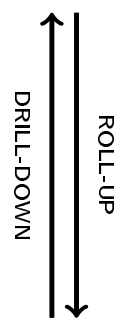

Nesse exemplo, as localizações de aeroportos não formam uma hierarquia e apenas a hierarquia de Hospital pode ser percorrida. Além disso, mesmo que os aeroportos não sejam referenciados por tabelas de fatos, é possível realizar junção espacial com as localizações de hospitais, desde que os hospitais são referenciados por tabelas de fatos. 


\subsubsection{Estratégia SJB}

Nesta seção é apresentada a estratégia Spatial Join Bitmap (SJB), a estratégia desenvolvida para processar consultas SOLAP com junção espacial utilizando o SB-index. A estratégia proposta é baseada em duas etapas principais. Na primeira, o esquema estrela e cada tabela de dimensão espacial são indexados. Na segunda etapa, o esquema estrela é processado para produzir um único subresultado que é agrupado e ordenado para obter o resultado final.

A estrutura de dados do SB-index consiste em um vetor unidimensional do tipo sbit-vector com $n$ entradas. Esse vetor é armazenado em um arquivo sequencial em disco. Portanto, para realizar a junção de duas relações espaciais de acordo com um predicado espacial, é necessário manusear dois arquivos. Para o manuseio dos dois arquivos, é adotada uma abordagem Nested Loop guiada, a qual percorre sequencialmente os dois arquivos do SB-index em um laço aninhado, ou seja, um arquivo é lido em um laço externo e o outro arquivo em um laço interno. Essa leitura é feita com auxílio de um guia que contém as páginas e as respectivas entradas que devem ser processadas, e é feita em unidades de página de disco. Cada página armazena no máximo $m$ entradas.

Suponha que a junção espacial seja realizada entre as relações espaciais $A$ e $B$. Na fase de filtragem, para cada página de $A$ lida no laço externo é realizada a leitura de todas as páginas de $B$ no laço interno. Em especial, a página com menos entradas deve ser posicionada no laço externo, de forma que sempre seja realizado um número menor de leituras de páginas de disco. Somente as entradas listadas no guia são testadas pelo predicado espacial por meio de seus MBRs. Esse guia é gerado em tempo de execução por meio de uma consulta ao índice Bitmap de junção, que selecionas as chaves primárias das entradas, aplicando os predicados convencionais da consulta. Isso reduz o custo de processamento do predicado espacial, descartando entradas que não satisfazem os predicados convencionais e pares de entradas que não estão presentes na tabela de fatos.

Se necessário, os pares de objetos espaciais que foram selecionados na fase de filtragem são enviados para a fase de refinamento, a qual seleciona os pares de objetos que atendem ao predicado espacial por meio de suas geometrias exatas. Na sequência, os pares de objetos são enviados para a transformação do predicado espacial em um predicado convencional. Caso o tipo de dados ou predicado espacial não necessite de refinamento, os pares de objetos espaciais são enviados diretamente para a transformação em um predicado convencional. Em ambos os casos, a transformação é realizada por meio das chaves primárias dos objetos espaciais selecionados.

Os Algoritmos 5 e 6 detalham a estratégia SJB. O Algoritmo 5, chamado SJBContruirĺndices, define a etapa de construção dos índices, a qual envolve tanto a construção do índice Bitmap de junção, quanto a construção do SB-index para cada tabela de dimensão espacial. O algoritmo recebe como entrada um esquema estrela $E E$ com $n$ tabelas de dimensão espacial. É criado um índice Bitmap de junção $I B J$ (linha 1), e para cada tabela de dimensão espacial 
$T D E_{j}$ é criado um SB-index (linhas 2 e 3). Como saída são gerados um índice Bitmap de junção $(I B J)$ e $n$ SB-index $\left(S B_{1}, \ldots, S B_{n}\right)$. O índice $I B J$ deve conter todos os atributos de $E E$ envolvidos nas consultas que serão executadas. Os $\mathrm{SB}$-index são construídos seguindo o algoritmo de construção original descrito na seção 4.4.1.

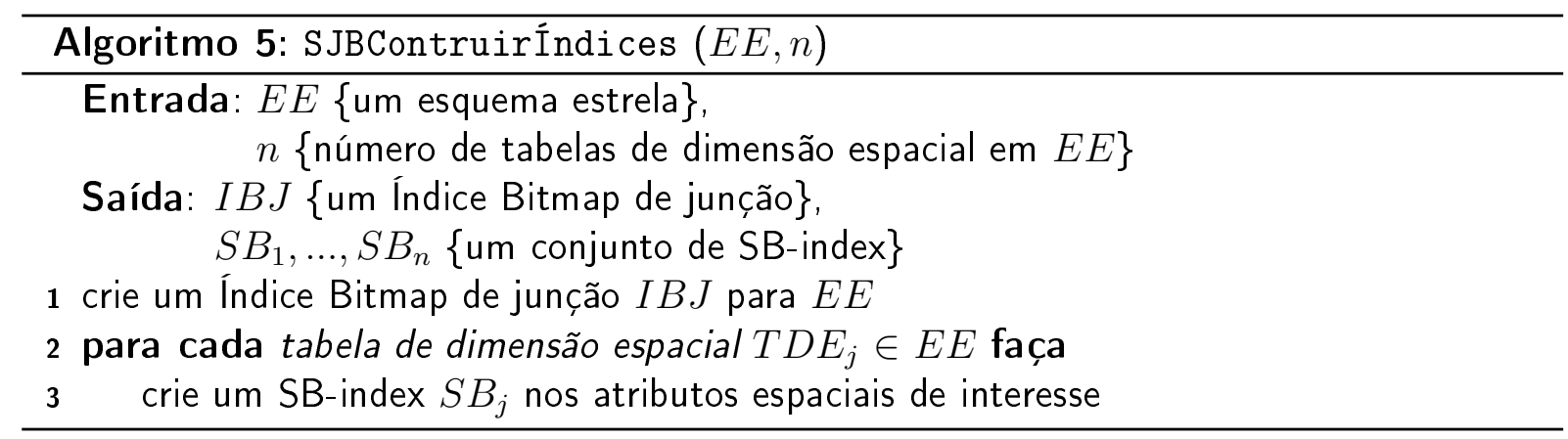

O Algoritmo 6, chamado SJBProcessarConsulta, define o processamento de consultas da estratégia SJB. O algoritmo recebe como entrada uma consulta SOLAP com junção espacial $Q$, um esquema estrela $E E$, um Índice Bitmap de junção $I B J$ e um conjunto de SB-index $S B$. O primeiro passo é a criação do guia que relaciona as páginas e entradas que são processadas pelo predicado espacial (linha 1 ). Um resultado parcial é gerado a partir da aplicação do SB-index na consulta $Q$ (linha 2). O resultado final da consulta SOLAP com junção espacial, saída do algoritmo, é obtido aplicando-se as operações de group by (linha 3) e order by (linha 4) de $Q$. O processamento do SB-index é realizado utilizando seu algoritmo original descrito na seção 4.4.2.

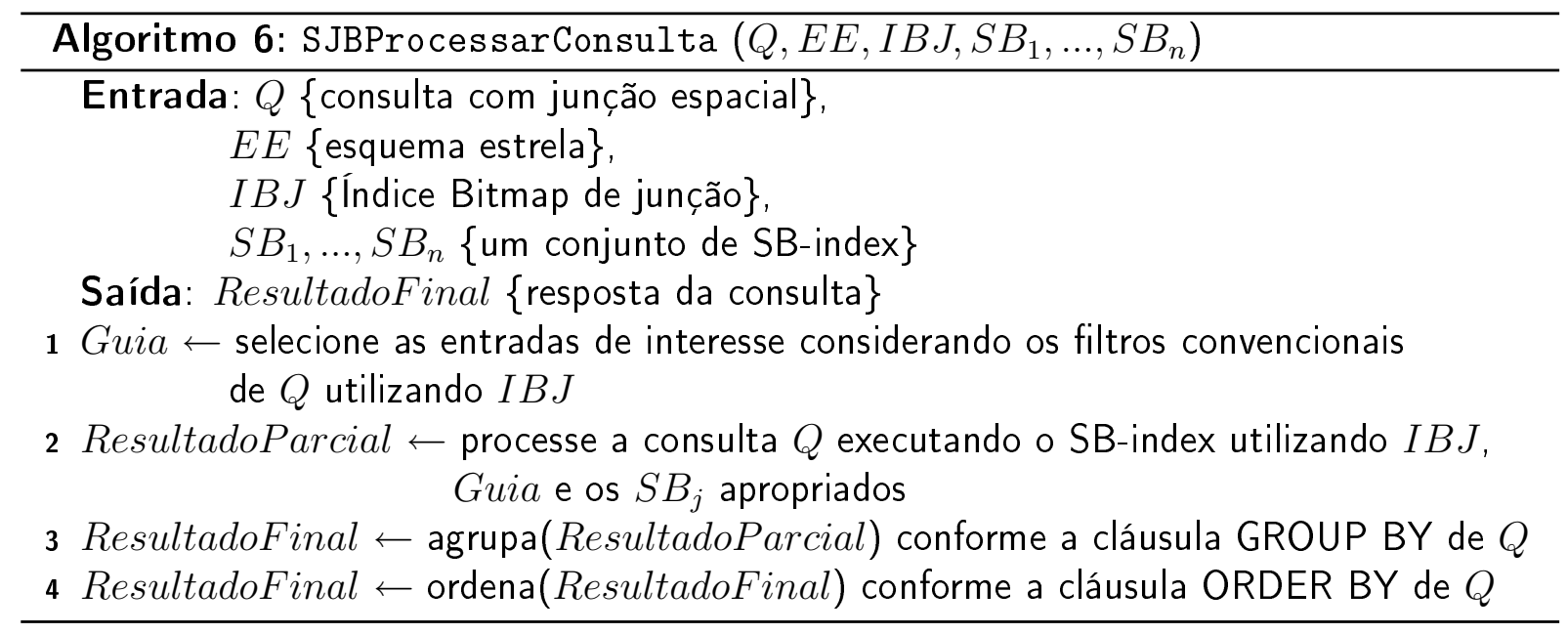

\subsection{Testes de Desempenho}

Nessa seção são apresentados os resultados obtidos nos testes de desempenho do processamento de consultas SOLAP com junção espacial utilizando a estratégia de processamento 
proposta. Antes da apresentação dos resultados, a seção 5.2.1 detalha a configuração dos testes.

\subsubsection{Configuração dos Testes}

Os seguintes aspectos relacionados à configuração dos testes foram definidos para a execução dos testes de desempenho: ambiente de testes, esquemas, dados, consultas, predicados convencionais e espaciais, e os métodos usados para comparação.

\section{$\underline{\text { Ambiente de Testes }}$}

Os testes foram conduzidos em uma máquina com um processador Intel Core i7 2,67 GHz, 12GB de memória RAM, 2 discos rígidos de $1 \mathrm{~TB}, 2$ discos rígidos de $2 \mathrm{~TB}$, Linux Ubuntu 11.10, kernel 3.0.0-13-generic-pae, PostgreSQL 9.1.1, PostGIS 1.5.3 e FastBit 1.2.4.

\section{Esquema}

Com base nos requisitos definidos na seção 6.1.1, nesta dissertação é proposta a utilização do esquema SpatialDrillAcross com a adição de duas tabelas com dados espaciais: River e Port. Esse esquema possui dimensões e dados convencionais e espaciais e permite que consultas SOLAP com junção espacial sejam processadas sobre seus dados. Na Figura 6.1 é ilustrado o esquema SpatialDrillAcross com as duas tabelas adicionais. Para facilitar a descrição dos experimentos, esse esquema é chamado de SpatialDrillAcross+.

O esquema SpatialDrillAcross+ possui duas hierarquias espaciais: $(i)$ (region_geo) $\preceq$ $($ nation_geo $) \preceq($ city_geo $) \preceq($ c_address_geo $) ;$ e $($ ii $)($ region_geo $) \preceq($ nation_geo $) \preceq$ (city_geo $\preceq\left(s_{-} a d d r e s s \_g e o\right)$. A hierarquia espacial de Supplier está relacionada às tabelas de fatos Partsupp e Lineitem. A hierarquia espacial de Customer está relacionada apenas à tabela Lineitem. As tabelas River e Port possuem dados espaciais sobre rios e portos, respectivamente, e não são referenciadas por nenhuma outra tabela do esquema.

\section{Dados}

O dados convencionais e espaciais utilizados são dados sintéticos. Dados convencionais foram gerados a partir do benchmark TPC-H. Dados espaciais referentes a endereços (pontos), cidades (polígonos), nações (polígonos) e regiões (polígonos) foram gerados a partir do benchmark Spadawan (Siqueira et al., 2010). Dados espaciais referentes a rios (linhas) e portos (pontos) foram gerados a partir de dados Geográficos do Brasil disponíveis na web ${ }^{1}$. Esses dados foram transladados e alguns rios foram descartados para diminuir a densidade. As cardinalidades das tabelas de dimensão espaciais City, Nation, Region, River e Port são controladas pelo fator de escala espacial SSF. Esse parâmetro permite explorar como o de-

\footnotetext{
${ }^{1}$ http://www.gismaps.com.br/divpol/divpol.htm
} 


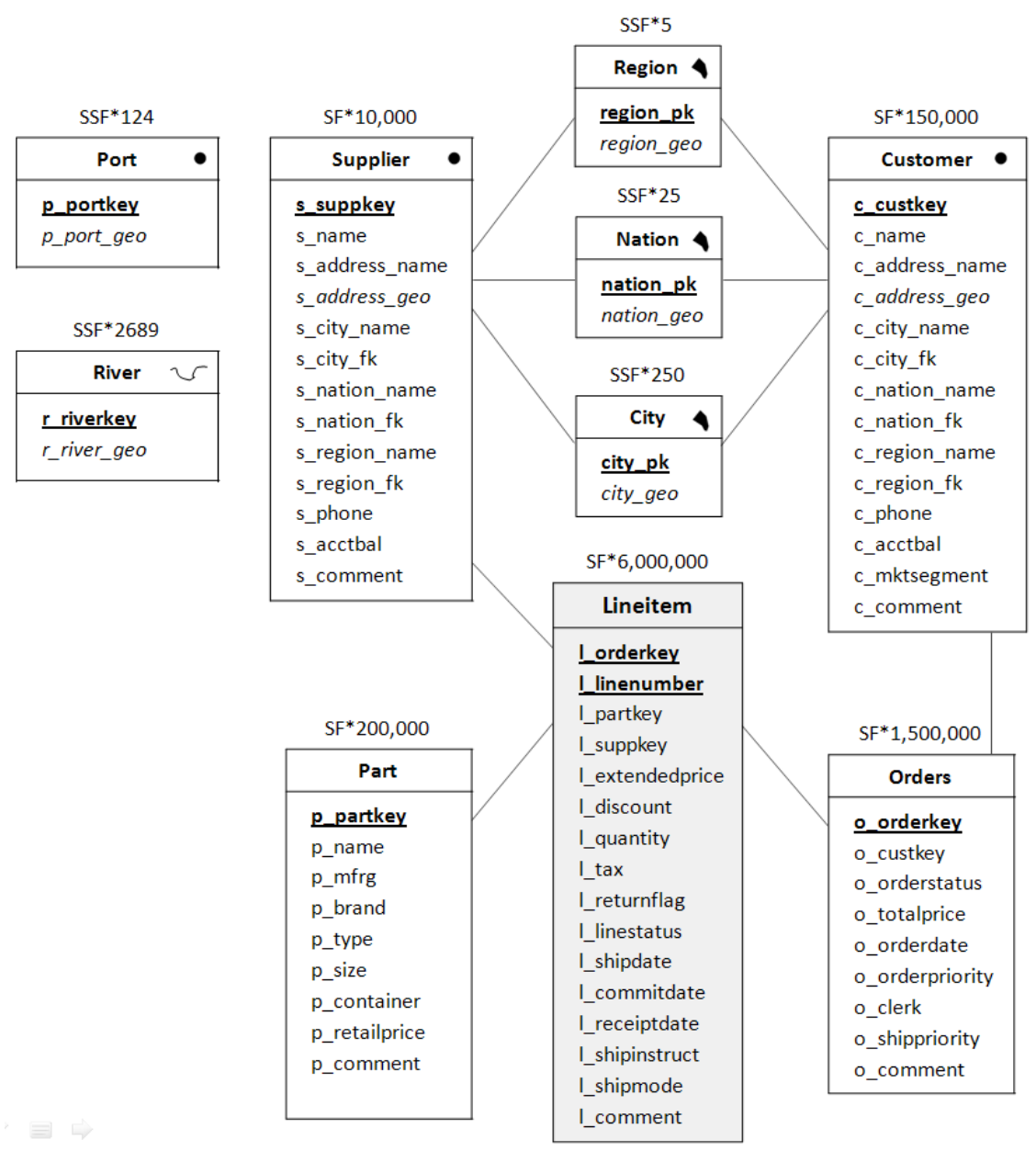

Figura 6.1: Esquema SpatialDrillAcross+.

sempenho das estratégias de processamento são afetadas mediante um fator de escala dos dados convencionais fixo. O aumento de dados espaciais é obtido por meio da replicação e translação do conjunto original de modo que não haja sobreposição. A Figura 6.2 ilustra os dados das tabelas City, Nation, Region, River e Port com $S S F=1$.

\section{Consultas}

As Figuras 6.3 e 6.4 ilustram as consultas utilizadas nos testes de desempenho. Essas consultas compõem, no total, 6 conjuntos, os quais são baseados no formato definido na seção 6.1.2. A Figura 6.3 ilustra o template das consultas, enquanto que a Figura 6.4 detalha os valores das variáveis granularidade e predicado espacial para cada consulta. De acordo com o template, todas as consultas compartilham as seguintes características:

- Utilizam a tabela de fatos Lineitem.

- Manipulam duas medidas numéricas, $l_{-}$discount e l_extendedprice. 

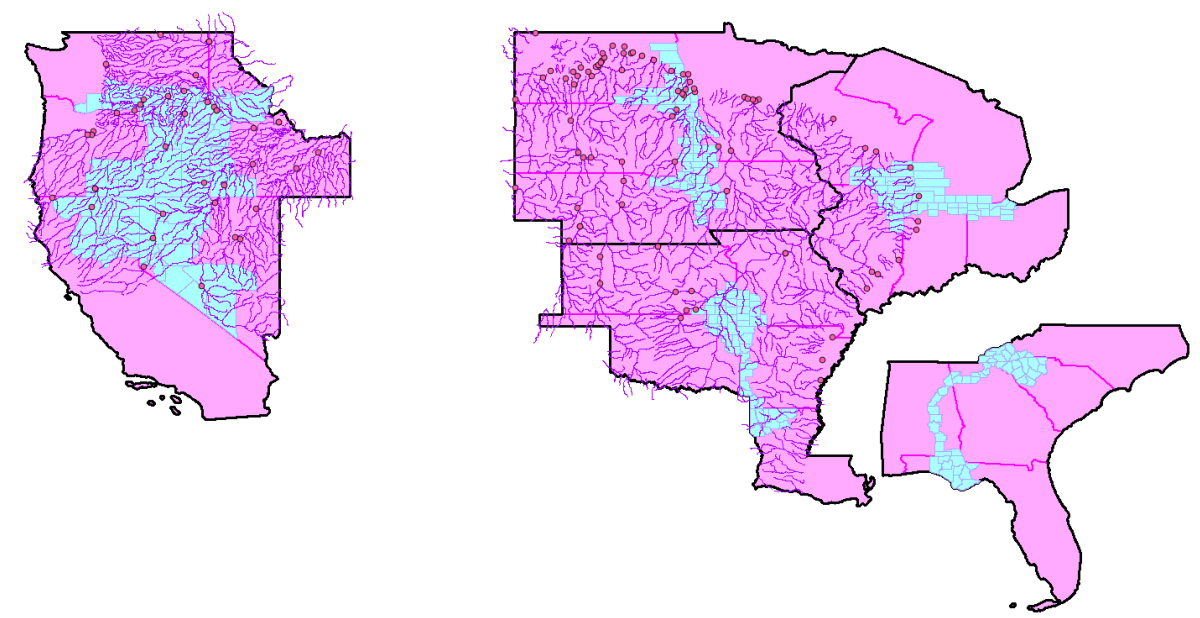

Figura 6.2: Dados espaciais das tabelas City, Nation, Region, River e Port com $S S F=1$.

- Recuperam dois atributos convencionais: $l \_y e a r$ e um atributo determinado pela variável granularidade, relacionado com a granularidade da consulta.

- Possuem os mesmos predicados convencionais.

- Possuem cláusula group by sobre os atributos convencionais recuperados.

- Possuem cláusula order by sobre os atributos convencionais recuperados.

As consultas Q1.1, Q1.2, Q1.3 e Q1.4 fazem parte do conjunto Q1. Elas possuem um predicado de junção espacial entre as hierarquias de Supplier e Customer, restringindo fornecedores

\section{SELECT granularidade,}

extract(year from I_shipdate) as I_year,

SUM(I_extendedprice*(1 - I_discount $))$ as revenue

FROM Supplier, Lineitem, Orders, Part, tabelas de dimensão espacial

WHERE I_partkey = p_partkey

AND I_suppkey $=$ s_suppkey

AND I_orderkey = o_orderkey

AND o_custkey = c_custkey

AND predicados convencionais

AND junções necessárias de acordo com o predicado espacial

AND predicado espacial

GROUP BY granularidade, I_year

ORDER BY granularidade, I year

Figura 6.3: Template dos conjuntos de consultas SOLAP com junção espacial. 


\begin{tabular}{|c|c|c|}
\hline Consulta & granularidade & predicado espacial \\
\hline Q1.1 & s_name, c_name & DISTANCE $\left(s \_a d d r e s s \_g e o, c \_a d d r e s s \_g e o\right)<=d$ \\
\hline Q1.2 & s_name, c_city_name & WITHIN(s_address_geo, c_city_geo $)$ \\
\hline Q1.3 & s_name, c_nation_name & WITHIN(s_address_geo, c_nation_geo $)$ \\
\hline Q1.4 & s_name, c_region_name & WITHIN(s_address_geo, c_region_geo $)$ \\
\hline
\end{tabular}

\begin{tabular}{|c|c|c|}
\hline Consulta & granularidade & predicado espacial \\
\hline Q2.1 & s_city_name, c_name & WITHIN(c_address_geo, s_city_geo) \\
\hline Q2.2 & s_city_name, c_city_name & INTERSECTS $\left(s \_c i t y \_g e o, c \_c i t y \_g e o\right)$ \\
\hline Q2.3 & s_city_name, c_nation_name & INTERSECTS(s_city_geo, c_nation_geo) \\
\hline Q2.4 & s_city_name, c_region_name & INTERSECTS(s_city_geo, c_region_geo) \\
\hline
\end{tabular}

\begin{tabular}{|c|l|l|}
\hline Consulta & \multicolumn{1}{|c|}{ granularidade } & \multicolumn{1}{c|}{ predicado espacial } \\
\hline Q3.1 & s_nation_name, c_name & WITHIN $\left(c \_a d d r e s s\right.$ geo, s_nation_geo $)$ \\
\hline Q3.2 & s_nation_name, c_city_name & INTERSECTS $\left(s \_n a t i o n \_g e o, c \_c i t y \_g e o\right)$ \\
\hline Q3.3 & s_nation_name, c_nation_name & INTERSECTS $\left(s \_n a t i o n \_g e o, c \_n a t i o n \_g e o\right)$ \\
\hline Q3.4 & s_nation_name, c_region_name & INTERSECTS $\left(s_{-}\right.$nation_geo,c_region_geo $)$ \\
\hline
\end{tabular}

\begin{tabular}{|c|l|l|}
\hline Consulta & \multicolumn{1}{|c|}{ granularidade } & \multicolumn{1}{c|}{ predicado espacial } \\
\hline Q4.1 & s_region_name, c_name & WITHIN $\left(c \_a d d r e s s \_g e o, s \_r e g i o n \_g e o\right)$ \\
\hline Q4.2 & s_region_name,c_city_name & INTERSECTS $\left(s \_r e g i o n \_g e o, c \_c i t y \_g e o\right)$ \\
\hline Q4.3 & s_region_name, c_nation_name & INTERSECTS $\left(s \_r e g i o n \_g e o, c \_n a t i o n \_g e o\right)$ \\
\hline Q4.4 & s_region_name, c_region_name & INTERSECTS $($ s_region_geo, c_region_geo $)$ \\
\hline
\end{tabular}

\begin{tabular}{|c|c|c|}
\hline Consulta & granularidade & predicado espacial \\
\hline Q5.1 & $s_{-}$name, c_name & $\begin{array}{l}\text { s_suppkey IN (select distinct } s \_s u p p k e y \text { from Supplier, Port } \\
\left.\text { where DISTANCE }\left(s \_a d d r e s s \_g e o, \text { port_geo }\right)<=d\right)\end{array}$ \\
\hline Q5.2 & s_city_name, c_name & $\begin{array}{l}\text { s_city_fk IN (select distinct city_pk from City, Port where } \\
\text { WITHIN }(\text { port_geo,city_geo }))\end{array}$ \\
\hline Q5.3 & s_nation_name, c_name & $\begin{array}{l}\text { s_nation_fk IN (select distinct nation_pk from Nation, Port } \\
\text { where WITHIN }(\text { port_geo,nation_geo }))\end{array}$ \\
\hline Q5.4 & s_region_name, c_name & $\begin{array}{l}\text { s_region_fk IN (select distinct region_pk from Region, Port } \\
\text { where WITHIN(port_geo,region_geo }) \text { ) }\end{array}$ \\
\hline
\end{tabular}

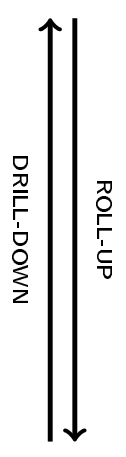

\begin{tabular}{|c|l|l|}
\hline Consulta & \multicolumn{1}{|c|}{ granularidade } & \multicolumn{1}{c|}{ predicado espacial } \\
\hline Q6.1 & s_city_name, c_name & $\begin{array}{l}s_{-} \text {city_f } k \text { IN (select distinct city_pk from City, River where } \\
\text { INTERSECTS }(\text { river_geo,city_geo }))\end{array}$ \\
\hline Q6.2 & s_nation_name, c_name & $\begin{array}{l}s_{-} \text {nation_f } k \text { IN (select distinct nation_pk from Nation, River } \\
\text { where INTERSECTS }(\text { river_geo,nation_geo }))\end{array}$ \\
\hline Q6.3 & s_region_name, c_name & $\begin{array}{l}s_{-} r e g i o n \_f k \text { IN (select distinct region_pk from Region, River } \\
\text { where INTERSECTS }(\text { river_geo,region_geo }))\end{array}$ \\
\hline
\end{tabular}

Figura 6.4: Valores de granularidade e predicado espacial dos conjuntos de consultas SOLAP com junção espacial Q1, Q2, Q3, Q4, Q5 e Q6. 
e clientes por meio de um relacionamento topológico entre suas localizações. Nesse conjunto a granularidade de Supplier é fixa, (s_address_geo). A hierarquia de Customer é variada da seguinte forma: Q1.1 em (c_address_geo), Q1.2 em (c_city_geo), Q1.3 em (c_nation_geo) e $\mathrm{Q} 1.4$ em (c_region_geo). Os atributos convencionais recuperados estão relacionados na $\mathrm{Fi}$ gura 6.4. Os conjuntos Q2, Q3 e Q4 diferenciam-se apenas pela granularidade da hierarquia de Supplier, que é mantida fixa nas seguintes granularidades: Q2 em (s_city_geo), Q3 em (s_nation_geo) e Q4 em (s_region_geo). Além disso, os tipos de relacionamentos topológicos utilizados variam de acordo com os tipos de dados envolvidos.

As consultas Q5.1, Q5.2, Q5.3 e Q5.4 fazem parte do conjunto Q5. Elas possuem um predicado de junção espacial entre a tabela espacial Port e a hierarquia espacial de Supplier, restringindo os fornecedores àqueles cujas localizações possuam um certo relacionamento topológico com as localizações dos portos. Nesse caso apenas a hierarquia de Supplier pode ser variada. Os predicados e atributos convencionais recuperados estão relacionados na Figura 6.4 .

As consultas Q6.1, Q6.2, Q6.3 fazem parte do conjunto Q6. Elas possuem um predicado de junção espacial entre a tabela espacial River e a hierarquia espacial de Supplier, restringindo os fornecedores àqueles cujas localizações possuam um certo relacionamento topológico com as localizações dos rios. Novamente, apenas a hierarquia de Supplier pode ser variada. Nesse conjunto a granularidade (s_address_geo) não é considerada. Os predicados e atributos convencionais recuperados estão relacionados na Figura 6.4.

\section{$\underline{\text { Predicados Convencionais e Espaciais }}$}

Nos testes de desempenho foram utilizados três configurações de predicados convencionais. Foram escolhidos três predicados para definir as três configurações. A escolha baseou-se nos atributos disponíveis e cardinalidade dos mesmos. Com relação à porcentagem aproximada de tuplas recuperadas pelas configurações: $C 1$ recupera 4,0\%, $C 2$ recupera 0,5\% e $C 3$ recupera $0,15 \%$. A Tabela 6.1 detalha as três configurações utilizadas nos testes de desempenho.

Tabela 6.1: Configurações dos predicados convencionais.

\begin{tabular}{|c|l|}
\hline Configuração & \multicolumn{1}{|c|}{ predicado convencional } \\
\hline$C 1$ & $p_{-}$brand='Brand\#14 ' \\
\hline$C 2$ & $p_{-}$brand='Brand\#14 ' and l_year=1995 \\
\hline$C 3$ & $p_{-}$brand='Brand\#14 ' and $l \_y e a r=1995$ and $l_{-} d i s c o u n t$ between 0.05 and 0.07 \\
\hline
\end{tabular}

Com relação aos predicados espaciais, nos testes de desempenhos foi fixado o valor $d=$ 0.25. Esse valor determina o raio de abrangência das consultas Q1.1 e Q5.1. As porcentagens 
de tuplas retornadas pelo predicado espacial de cada consulta nas respectivas bases de dados são listada na Tabela 6.2 .

Tabela 6.2: Porcentagem de tuplas retornadas pelo predicado espacial de cada consulta.

\begin{tabular}{|c|c|c|c|c|c|c|c|c|c|}
\hline Consulta & $\begin{array}{c}S F=5 \\
S S F=10\end{array}$ & $\begin{array}{l}S F=10 \\
S S F=5\end{array}$ & $\begin{array}{c}S F=10 \\
S S F=10\end{array}$ & $\begin{array}{c}S F=10 \\
S S F=15\end{array}$ & $\begin{array}{c}S F=10 \\
S S F=20\end{array}$ & $\begin{array}{c}S F=15 \\
S S F=10\end{array}$ & $\begin{array}{c}S F=20 \\
S S F=10\end{array}$ & $\begin{array}{c}S F=25 \\
S S F=10\end{array}$ & $\begin{array}{c}S F=30 \\
S S F=10\end{array}$ \\
\hline Q1.1 & $0.04 \%$ & $0.07 \%$ & $0.04 \%$ & $0.02 \%$ & $0.02 \%$ & $0.04 \%$ & $0.04 \%$ & $0.04 \%$ & $0.04 \%$ \\
\hline Q1.2 & $0.04 \%$ & $0.08 \%$ & $0.04 \%$ & $0.03 \%$ & $0.02 \%$ & $0.04 \%$ & $0.04 \%$ & $0.04 \%$ & $0.04 \%$ \\
\hline Q1.3 & $0.40 \%$ & $0.80 \%$ & $0.40 \%$ & $0.27 \%$ & $0.20 \%$ & $0.40 \%$ & $0.40 \%$ & $0.40 \%$ & $0.40 \%$ \\
\hline Q1.4 & $2.00 \%$ & $4.00 \%$ & $2.00 \%$ & $1.33 \%$ & $1.00 \%$ & $2.00 \%$ & $2.00 \%$ & $2.00 \%$ & $2.00 \%$ \\
\hline Q2.1 & $0.04 \%$ & $0.08 \%$ & $0.04 \%$ & $0.03 \%$ & $0.02 \%$ & $0.04 \%$ & $0.04 \%$ & $0.04 \%$ & $0.04 \%$ \\
\hline Q2.2 & $0.20 \%$ & $0.41 \%$ & $0.21 \%$ & $0.14 \%$ & $0.10 \%$ & $0.21 \%$ & $0.21 \%$ & $0.21 \%$ & $0.21 \%$ \\
\hline Q2.3 & $0.75 \%$ & $1.49 \%$ & $0.74 \%$ & $0.50 \%$ & $0.37 \%$ & $0.75 \%$ & $0.74 \%$ & $0.74 \%$ & $0.75 \%$ \\
\hline Q2.4 & $2.00 \%$ & $4.00 \%$ & $2.00 \%$ & $1.33 \%$ & $1.00 \%$ & $2.00 \%$ & $2.00 \%$ & $2.00 \%$ & $2.00 \%$ \\
\hline Q3.1 & $0.40 \%$ & $0.80 \%$ & $0.40 \%$ & $0.27 \%$ & $0.20 \%$ & $0.40 \%$ & $0.40 \%$ & $0.40 \%$ & $0.40 \%$ \\
\hline Q3.2 & $0.75 \%$ & $1.49 \%$ & $0.74 \%$ & $0.50 \%$ & $0.37 \%$ & $0.74 \%$ & $0.75 \%$ & $0.74 \%$ & $0.75 \%$ \\
\hline Q3.3 & $1.71 \%$ & $3.42 \%$ & $1.71 \%$ & $1.14 \%$ & $0.85 \%$ & $1.71 \%$ & $1.71 \%$ & $1.71 \%$ & $1.71 \%$ \\
\hline Q3.4 & $2.88 \%$ & $5.75 \%$ & $2.88 \%$ & $1.92 \%$ & $1.44 \%$ & $2.88 \%$ & $2.88 \%$ & $2.88 \%$ & $2.88 \%$ \\
\hline Q4.1 & $2.00 \%$ & $4.00 \%$ & $2.00 \%$ & $1.33 \%$ & $1.00 \%$ & $2.00 \%$ & $2.00 \%$ & $2.00 \%$ & $2.00 \%$ \\
\hline Q4.2 & $2.00 \%$ & $4.00 \%$ & $2.00 \%$ & $1.33 \%$ & $1.00 \%$ & $2.00 \%$ & $2.00 \%$ & $2.00 \%$ & $2.00 \%$ \\
\hline Q4.3 & $2.88 \%$ & $5.76 \%$ & $2.88 \%$ & $1.92 \%$ & $1.44 \%$ & $2.88 \%$ & $2.88 \%$ & $2.88 \%$ & $2.88 \%$ \\
\hline Q4.4 & $4.40 \%$ & $8.80 \%$ & $4.40 \%$ & $2.93 \%$ & $2.20 \%$ & $4.40 \%$ & $4.40 \%$ & $4.40 \%$ & $4.40 \%$ \\
\hline Q5.1 & $3.93 \%$ & $3.98 \%$ & $3.94 \%$ & $3.96 \%$ & $3.90 \%$ & $3.94 \%$ & $3.91 \%$ & $3.95 \%$ & $3.87 \%$ \\
\hline Q5.2 & $9.20 \%$ & $9.19 \%$ & $9.19 \%$ & $9.19 \%$ & $9.19 \%$ & $9.20 \%$ & $9.20 \%$ & $9.20 \%$ & $9.20 \%$ \\
\hline Q5.3 & $71.99 \%$ & $72.00 \%$ & $72.00 \%$ & $72.00 \%$ & $72.00 \%$ & $72.00 \%$ & $72.00 \%$ & $72.00 \%$ & $71.99 \%$ \\
\hline Q5.4 & $79.99 \%$ & $80.00 \%$ & $80.00 \%$ & $80.00 \%$ & $80.00 \%$ & $80.01 \%$ & $80.00 \%$ & $80.00 \%$ & $79.99 \%$ \\
\hline Q6.1 & $66.79 \%$ & $66.80 \%$ & $66.80 \%$ & $66.80 \%$ & $66.80 \%$ & $66.80 \%$ & $66.80 \%$ & $66.80 \%$ & $66.79 \%$ \\
\hline Q6.2 & $76.00 \%$ & $76.00 \%$ & $76.00 \%$ & $76.00 \%$ & $76.00 \%$ & $76.00 \%$ & $75.99 \%$ & $76.00 \%$ & $75.99 \%$ \\
\hline Q6.3 & $79.99 \%$ & $80.00 \%$ & $80.00 \%$ & $80.00 \%$ & $80.00 \%$ & $80.01 \%$ & $80.00 \%$ & $80.00 \%$ & $79.99 \%$ \\
\hline
\end{tabular}

\section{Métodos para Comparação}

Os métodos usados para comparar e validar a estratégia proposta foram a junção estrela e visões materializadas. Em especial, o índice espacial R-tree foi utilizado para indexar os dados espaciais tanto da junção estrela quando das visões materializadas. Na contrução do índice Bitmap de junção pelo software FastBit, foram utilizadas as opções <binning none/> e <encoding equality/>. Isso significa que a técnica de otimização binning não foi utilizada e que foi contruído um vetor Bitmap para cada valor de todos os atributos. 


\section{Medida Coletada}

Nos testes de desempenho foi coletado o tempo gasto em segundos na execução das consultas. O tempo de cada consulta corresponde à média de 5 execuções, sendo que entre cada execução foi realizada a limpeza da memória cache.

Os tempos de execução da junção estrela e da visão materializada correspondem ao melhor tempo obtido em duas configurações, conforme descrito a seguir. Na primeira, os atributos envolvidos nos predicados convencionais ( $p$ brand, $l \_$year e $l_{-}$discount) foram indexados por meio de B-trees. Já na segunda configuração não foram usados índices.

\subsubsection{Comparação entre Junção Estrela, Visão Materializada e a Estratégia SJB}

O objetivo desse teste foi comparar o desempenho da estratégia proposta SJB com o uso da junção estrela e de visão materializada. A visão materializada considerada no teste é a visão utilizada na criação do índice Bitmap de junção pela estratégia SJB.

No teste foi utilizada uma base fixa gerada a partir do esquema SpatialDrillAcross + com a seguinte configuração: $S F=10$ e $S S F=10$. Foram utilizadas as três configurações de predicados, $C 1, C 2$ e $C 3$. Para fins de melhor visualização dos resultados, nos gráficos exibidos nessa seção é ilustrada a razão entre o tempo gasto pela estratégia SJB $\left(T_{S J B}\right)$ e o

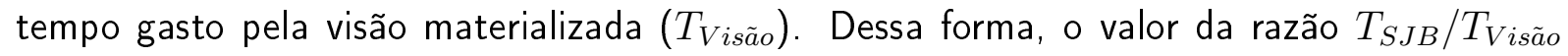
menor do que 1 indica que a estratégia SJB é mais eficiente.

Os resultados desse teste de desempenho são exibidos nas Figuras 6.5 e 6.6. Os gráficos mostram que a estratégia SJB obteve desempenho superior à junção estrela em todas as consultas. Comparada à junção estrela, a estratégia SJB mostrou uma redução no tempo de $0.3 \%$ a $98,9 \%$ na configuração $C 1$, de $29,3 \%$ a $96,5 \%$ na configuração $C 2$ e de $49,0 \%$ a $99,2 \%$ na configuração $C 3$.

Com relação à visão materializada, a estratégia SJB garantiu sempre o melhor desempenho na configuração $C 3$, mostrando uma redução no tempo de $2,5 \%$ a $71,4 \%$. Considerando $C 1$, a estratégia SJB perdeu em desempenho para a visão materializada nas consultas Q1.1, Q1.3, Q1.4, Q2.1, Q3.1 e Q4.1. Este comportamento já era previsto, visto que essas consultas são exatamente as que geram um custo alto de processamento pelo índice Bitmap de junção. Isso ocorre porque seus predicados espaciais possuem altas seletividades, além de envolverem atributos de alta cardinalidade, fazendo com que muitos vetores Bitmap sejam lidos e processados. Com isso, operações de leituras sobre as tabelas tornam-se menos custosas que a utilização do índice Bitmap de junção. Nas consultas restantes, a estratégia SJB mostrou uma redução no tempo de $12,7 \%$ a $96,4 \%$.

Na configuração $C 2$, a estratégia SJB foi superada pela visão materializada somente na consulta Q4.1. No restante das consultas, a estratégia SJB mostrou uma redução no tempo de $5,8 \%$ a $70,0 \%$. Esse desempenho crescente da estratégia SJB com relação às diferentes 
configurações se deve ao fato de que conforme os predicados convencionais recuperam menos tuplas, mais a estratégia filtra e elimina candidatos, diminuindo significativamente o custo de processamento da consulta transformada pelo índice Bitmap de junção.

Portanto, a estratégia proposta é uma excelente opção como estratégia de processamento para consultas SOLAP com junção espacial, principalmente para consultas com predicados convencionais de baixa seletividade.

Nos próximos testes, como a junção estrela garantiu sempre o pior desempenho em todas as consultas, ela não será considerada.

\subsubsection{SJB: Variando o Fator de Escala Convencional}

O objetivo deste teste foi comparar o desempenho da estratégia SJB e da visão materializada quando o fator de escala convencional (parâmetro $S F$ ) é variado. Com a variação desse parâmetro é possível verificar como essas duas abordagens se comportam na presença de um volume crescente de dados convencionais em todas as consultas, e de dados espaciais para consultas relacionadas às granularidades (s_address_geo) e (c_address_geo).

No teste foram utilizadas seis bases geradas a partir do esquema SpatialDrillAcross+. O parâmetro $S S F$ foi mantido fixo com um valor igual a 10 , enquanto que o parâmetro $S F$ foi variado com o objetivo de aumentar o volume de dados convencionais, sendo utilizados os seguintes valores: 5, 10, 15, 20, 25 e 30. Foram utilizadas as três configurações de predicados, $C 1, C 2$ e $C 3$. Novamente, é exibida nos gráficos a razão $T_{S J B} / T_{V i s a ̃ o}$.

Os resultados desse teste de desempenho são exibidos nas Figuras 6.7 e 6.8. Esses resultados corroboram os resultados obtidos no teste anterior, isto é, a estratégia SJB obteve desempenho semelhante se forem consideradas as mesmas consultas e as suas respectivas configurações. Em detalhes, a estratégia SJB obteve sempre o melhor desempenho na configuração $C 3$, mostrando uma redução no tempo de $1,0 \%$ a $85,1 \%$. Consultas dos conjuntos Q1, Q2, Q3 e Q4 são mais beneficiadas pela diminuição progressiva das porcentagem de tuplas retornadas pelas configurações de predicados convencionais. Para essas consultas, a redução do custo de processamento do predicado espacial é significativa. Já para os conjuntos Q5 e Q6, o tempo gasto pela estratégia SJB em cada consulta permaneceu praticamente constante, fazendo com que a razão aumentasse na configuração $C 3$. Esse aumento é causado pela diminuição do tempo gasto pela visão materializada, indicando que para consultas desse tipo, a visão consegue gerenciar melhor os predicados convencionais.

Como pode ser observado pelos resultados apresentados nessa seção, a diferença de desempenho entre as três configurações é muito pequena, e os valores da razão $T_{S J B} / T_{V i s a ̃ o}$ ainda indicam que a estratégia proposta é a melhor opção. 


\subsubsection{SJB: Variando o Fator de Escala Espacial}

O objetivo deste teste foi comparar o desempenho da estratégia SJB e da visão materializada quando o fator de escala espacial (parâmetro $S S F$ ) é variado. Com a variação do parâmetro $S S F$ é possível verificar como essas duas abordagens se comportam na presença de um volume crescente de dados espaciais nas tabelas City, Nation, Region, River e Port, isto é, exceto para as consultas Q1.1 que não se relacionam com essas tabelas.

No teste foram utilizadas quatro bases geradas a partir do esquema SpatialDrillAcross+. Foi usado o valor de $S F=10$, enquanto que o parâmetro $S S F$ foi variado de 5 a 20 , em passos de 5. Foram utilizadas as três configurações de predicados, $C 1, C 2$ e $C 3$. Novamente,

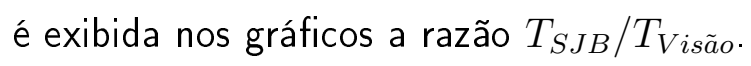

Os resultados desse teste de desempenho são exibidos nas Figuras 6.9 e 6.10. Mais uma vez, os resultados obtidos anteriormente foram corroborados, ou seja, a estratégia SJB obteve desempenho semelhante aos desempenhos anteriores se forem consideradas as mesmas consultas e as suas respectivas configurações. Portanto, as mesmas discussões realizadas nas seções 6.2.2 e 6.2.3 aplicam-se a essa seção. Com relação apenas à configuração C3, a estratégia SJB obteve sempre o melhor desempenho, exceto no ponto $S S F=5$ da consulta Q4.1. Desconsiderando essa consulta, a estratégia SJB mostrou uma redução no tempo de $2,5 \%$ a $88,3 \%$.

Relacionada ao objetivo desse teste, uma característica que pode ser observada nos gráficos é que quando o predicado espacial envolve conjuntos de objetos espaciais com cardinalidades alta (Q1,1, Q2,1, Q3,1 e Q4.1), as curvas tendem a apresentar um declínio conforme SSF aumenta. Isso indica que o aumento da cardinalidade nessas consultas afeta mais a visão materializada. Outra característica é que consultas nas quais as cardinalidades dos objetos são baixas (Q4.3, Q4.4, Q5.3 e Q5.4) apresentaram as menores média da razão $T_{S J B} / T_{V i s a ̃ o}$. 


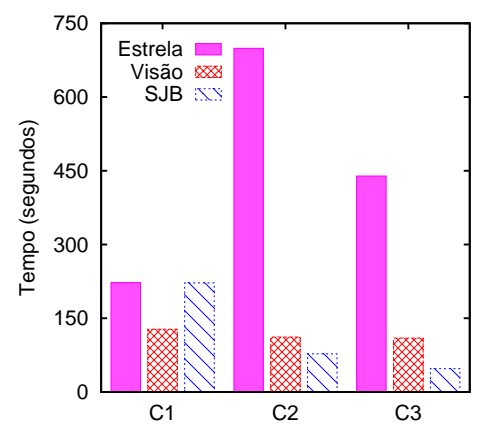

(a) Consulta Q1.1.

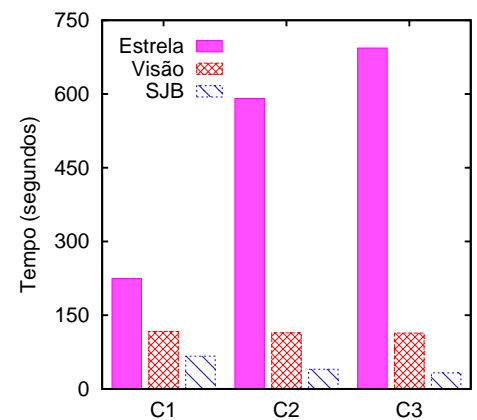

(b) Consulta Q1.2.

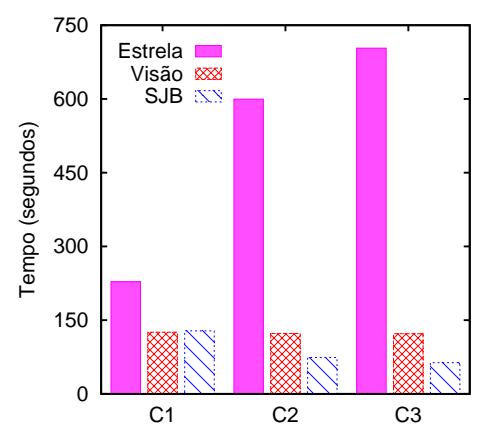

(c) Consulta Q1.3.

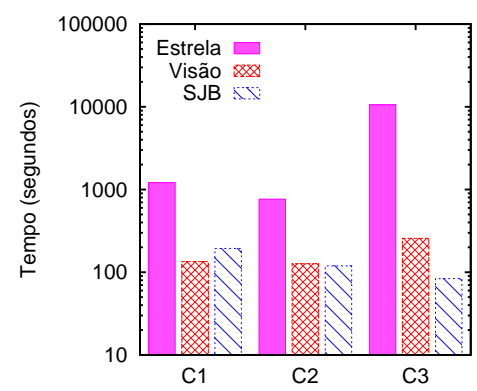

(d) Consulta Q1.4.

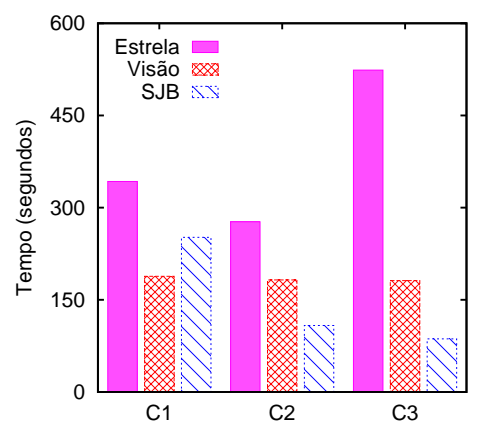

(e) Consulta Q2.1.

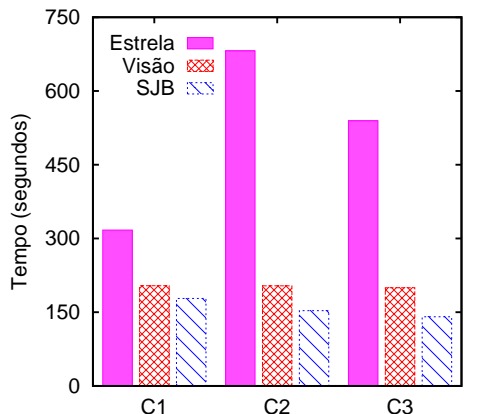

(f) Consulta Q2.2.

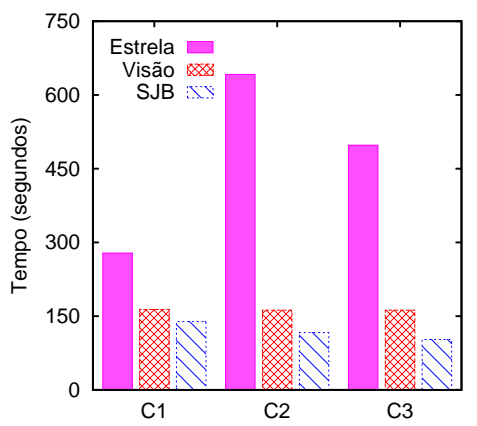

(g) Consulta Q2.3.

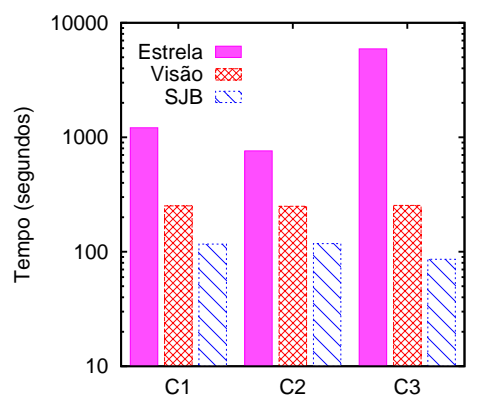

(h) Consulta Q2.4.

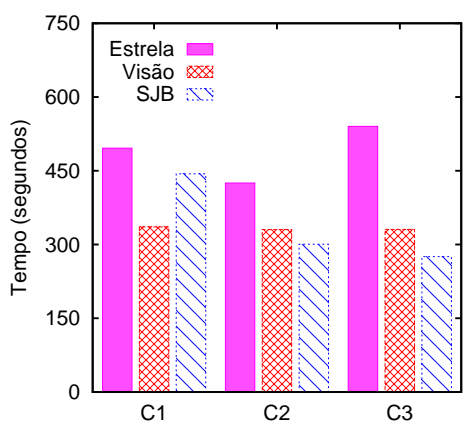

(i) Consulta Q3.1.

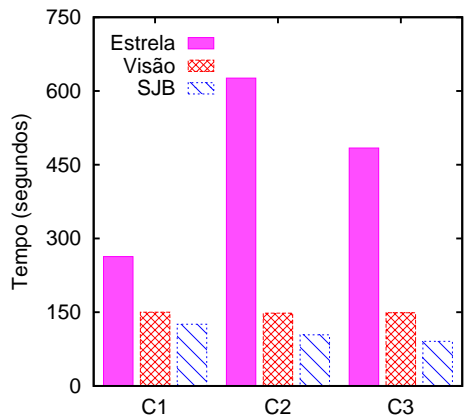

(j) Consulta Q3.2.

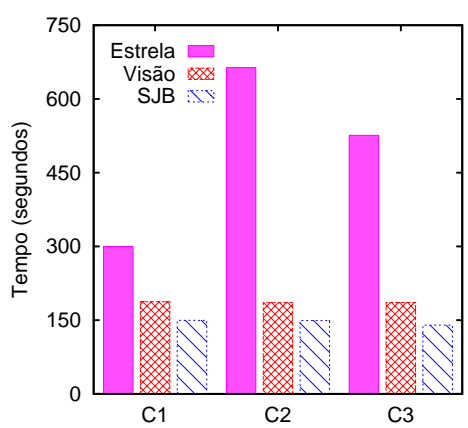

(k) Consulta Q3.3.

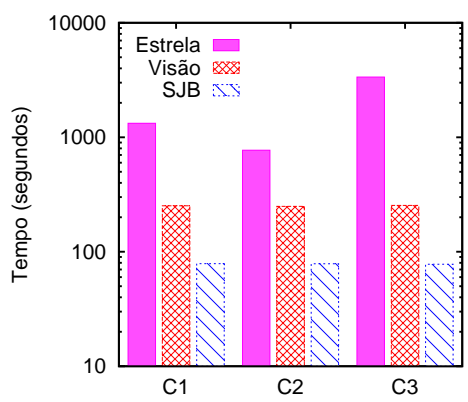

(I) Consulta Q3.4.

Figura 6.5: Comparação do desempenho entre junção estrela, visão materializada e a estratégia SJB para os conjuntos Q1, Q2 e Q3. O eixo y correspondente ao tempo de execução está em escala logarítmica nas consultas Q1.4, Q2.4 e Q3.4. 


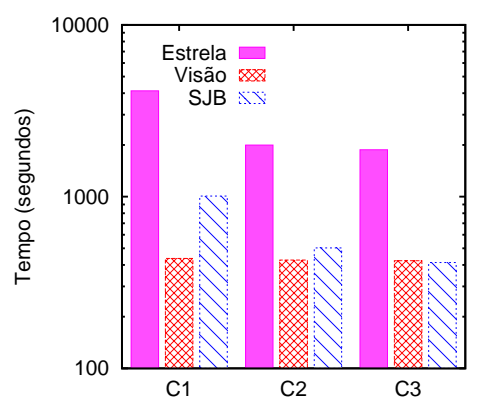

(a) Consulta Q4.1.

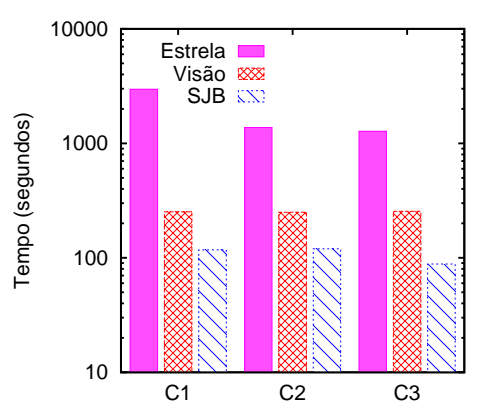

(b) Consulta Q4.2.

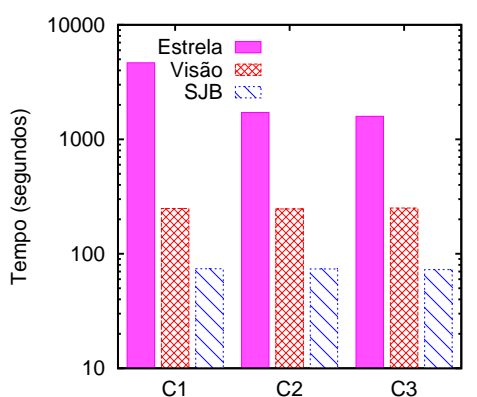

(c) Consulta Q4.3.

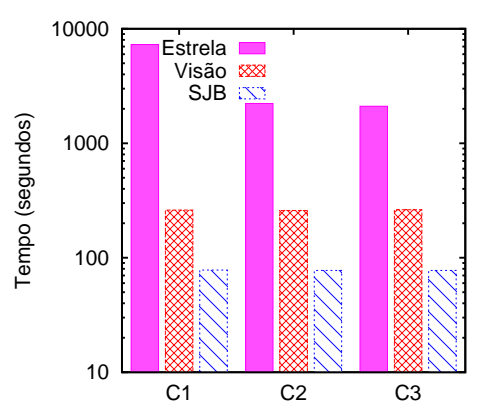

(d) Consulta Q4.4.

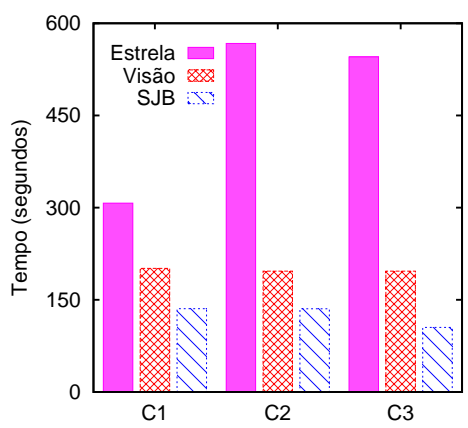

(e) Consulta Q5.1.

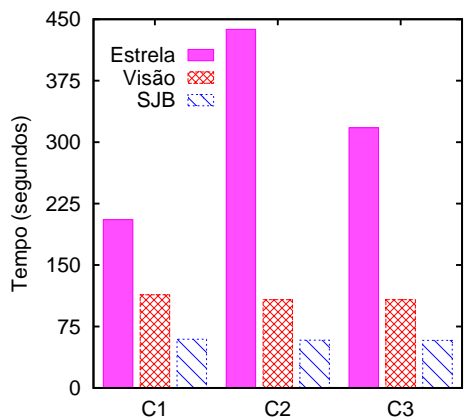

(f) Consulta Q5.2.

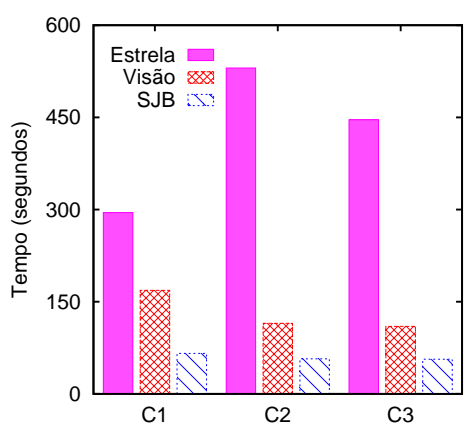

(g) Consulta Q5.3.

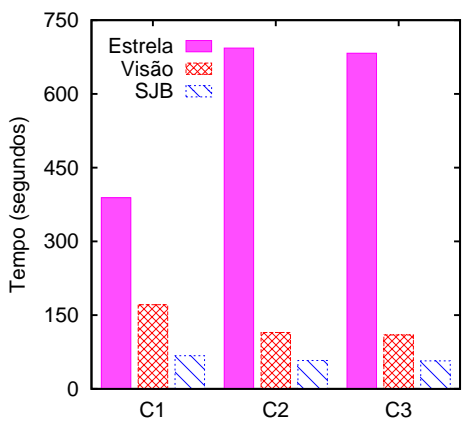

(h) Consulta Q5.4.

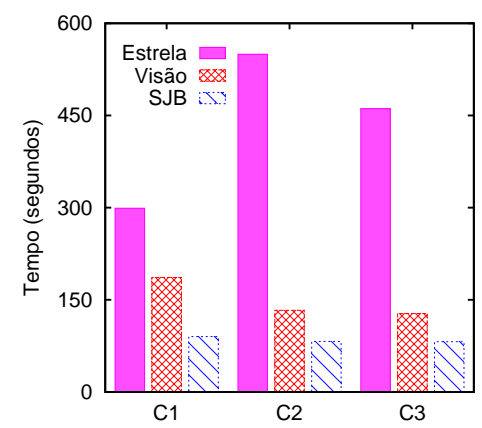

(i) Consulta Q6.1.

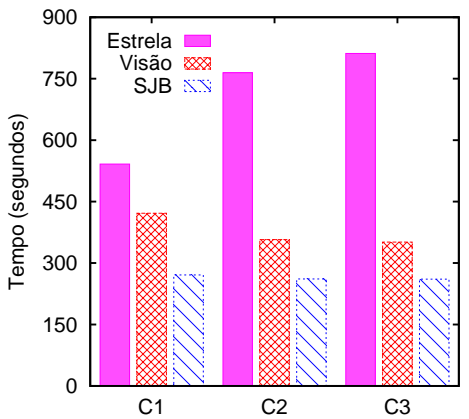

(j) Consulta Q6.2.

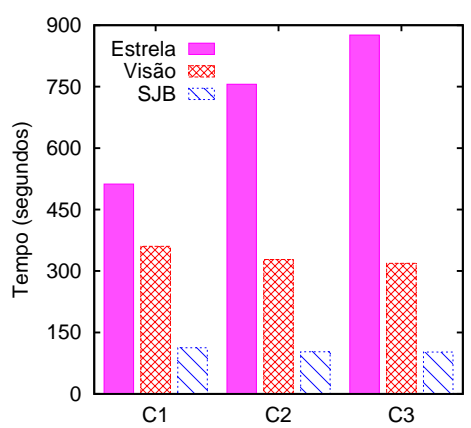

(k) Consulta Q6.3.

Figura 6.6: Comparação do desempenho entre junção estrela, visão materializada e a estratégia SJB para os conjuntos Q4, Q5 e Q6. O eixo y correspondente ao tempo de execução está em escala logarítmica nas consultas Q4.1, Q4.2, Q4.3, Q4.4. 


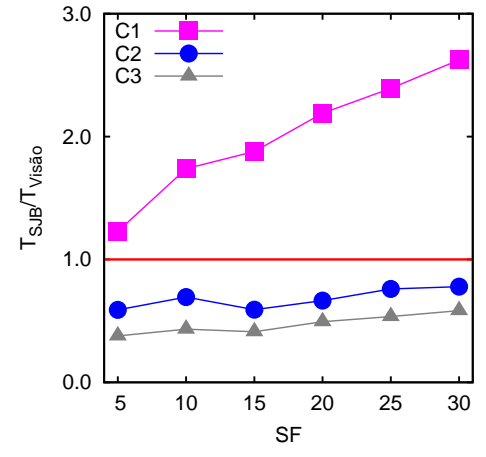

(a) Consulta Q1.1.

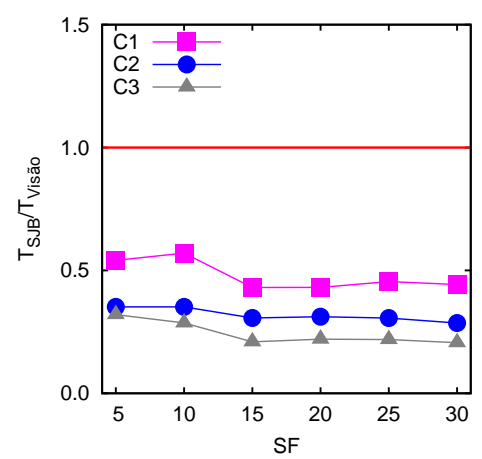

(b) Consulta Q1.2.

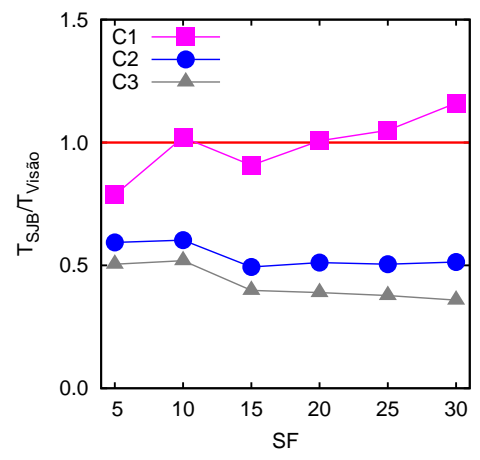

(c) Consulta Q1.3.

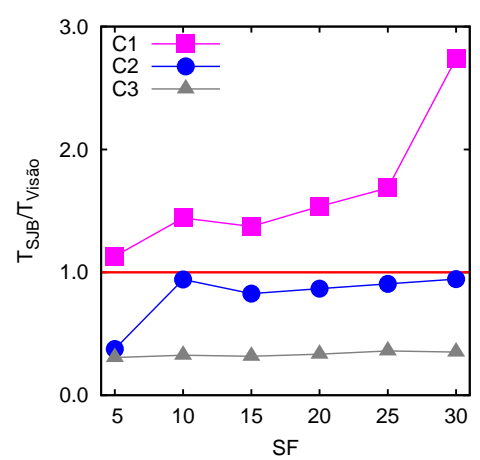

(d) Consulta Q1.4.

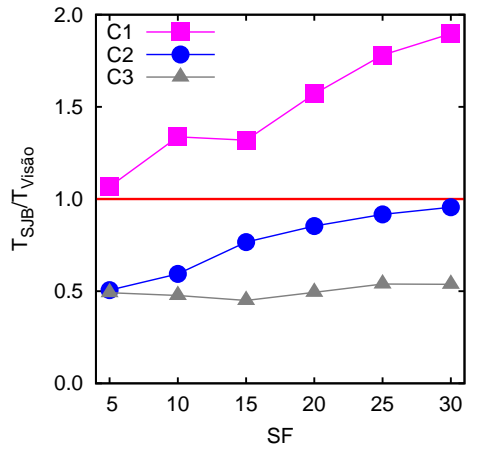

(e) Consulta Q2.1.

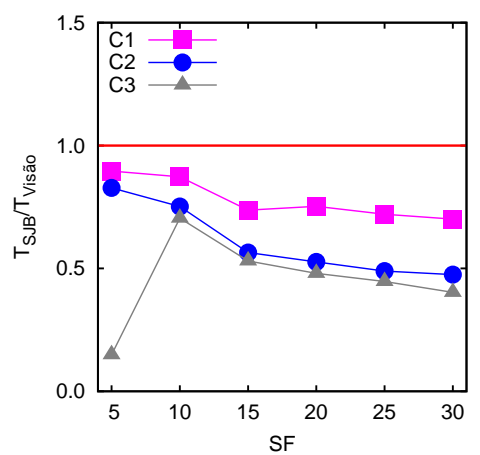

(f) Consulta Q2.2.

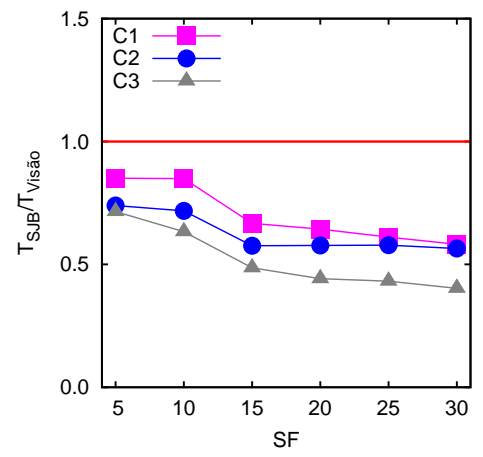

(g) Consulta Q2.3.

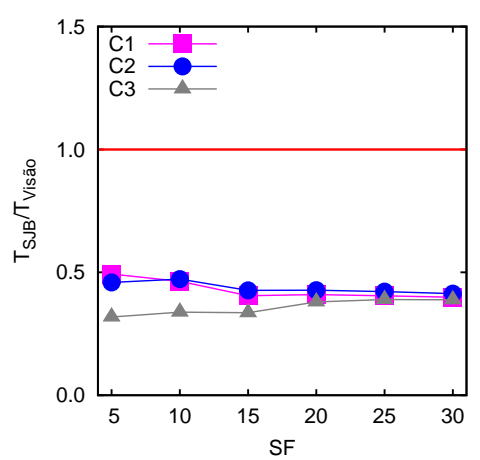

(h) Consulta Q2.4.

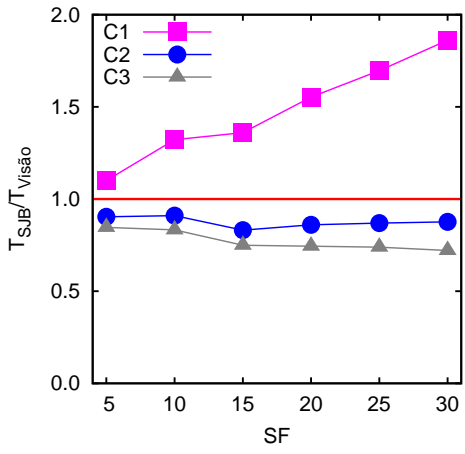

(i) Consulta Q3.1.

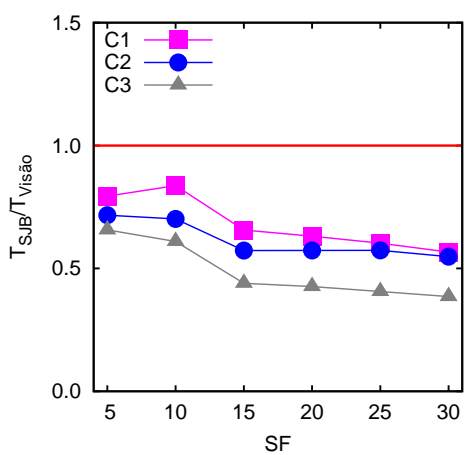

(j) Consulta Q3.2.

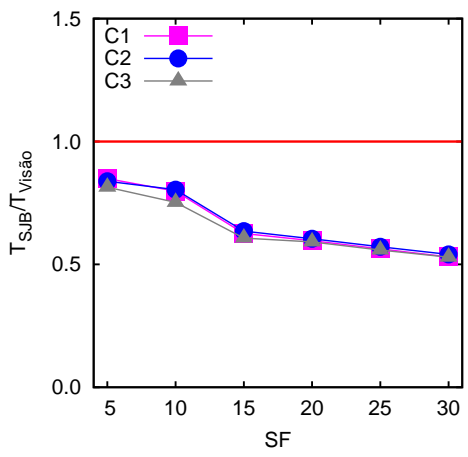

(k) Consulta Q3.3.

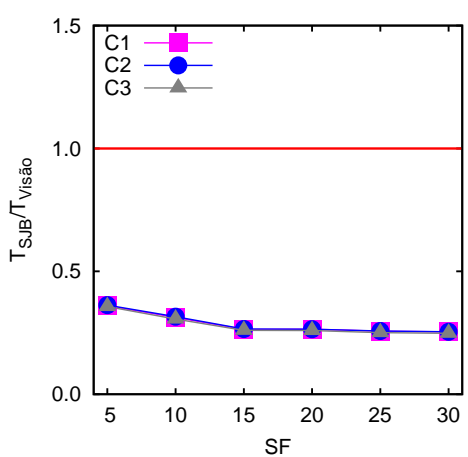

(I) Consulta Q3.4.

Figura 6.7: Comparação do desempenho entre visão materializada e a estratégia SJB para os conjuntos Q1, Q2 e Q3 variando o fator de escala (SF) convencional. 


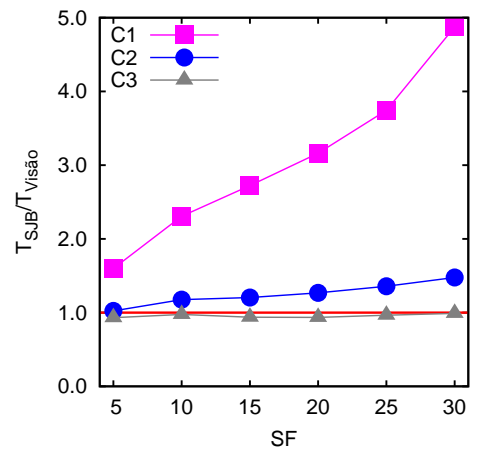

(a) Consulta Q4.1.

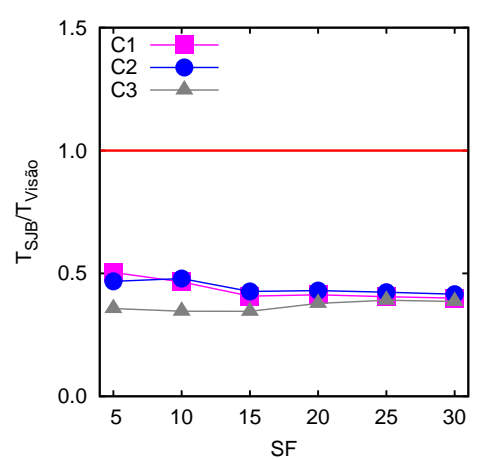

(b) Consulta Q4.2.

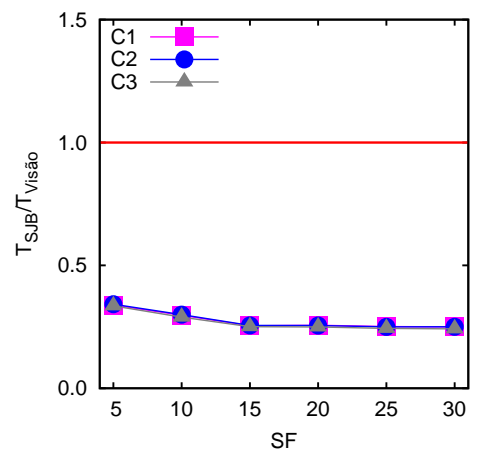

(c) Consulta Q4.3.

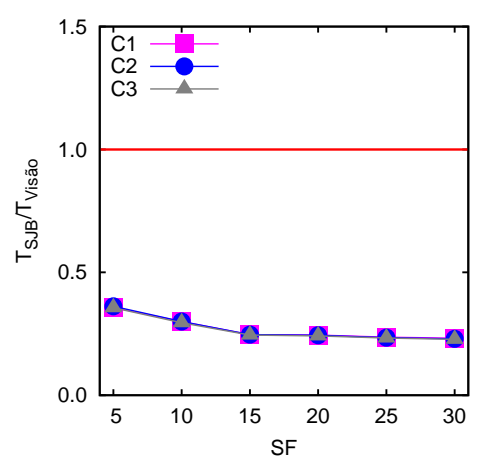

(d) Consulta Q4.4.

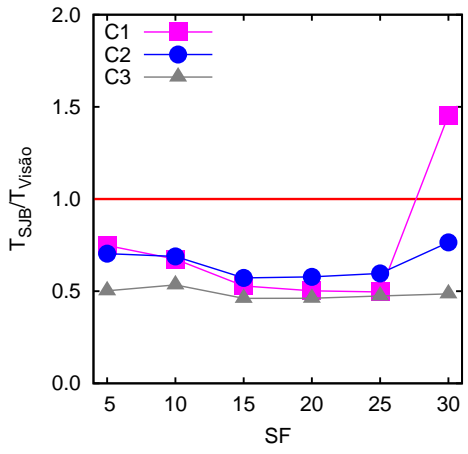

(e) Consulta Q5.1.

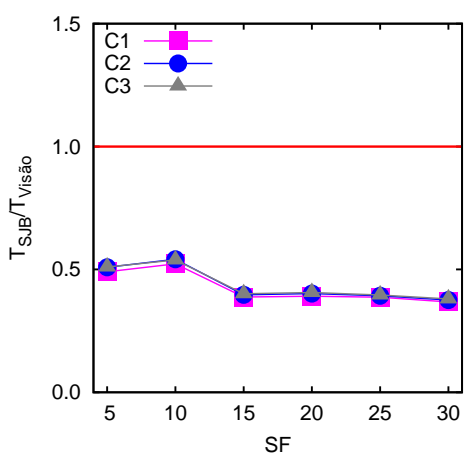

(f) Consulta Q5.2.

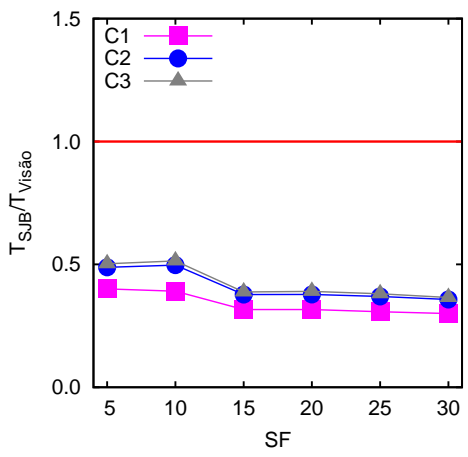

(g) Consulta Q5.3.

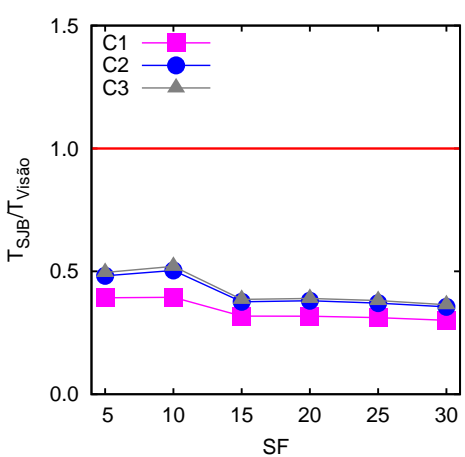

(h) Consulta Q5.4.

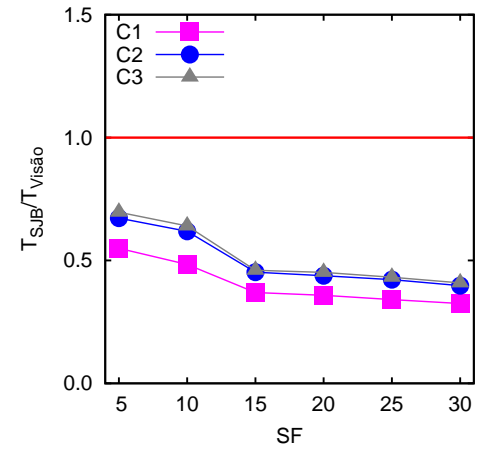

(i) Consulta Q6.1.

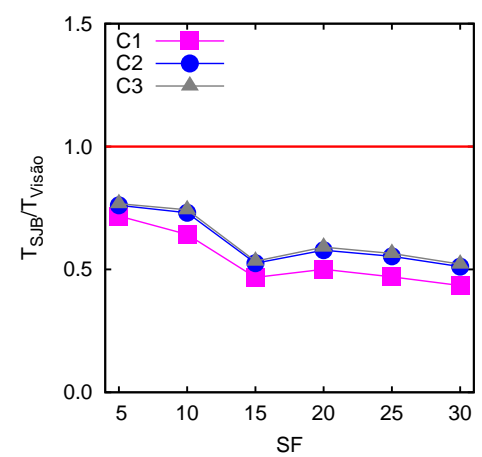

(j) Consulta Q6.2.

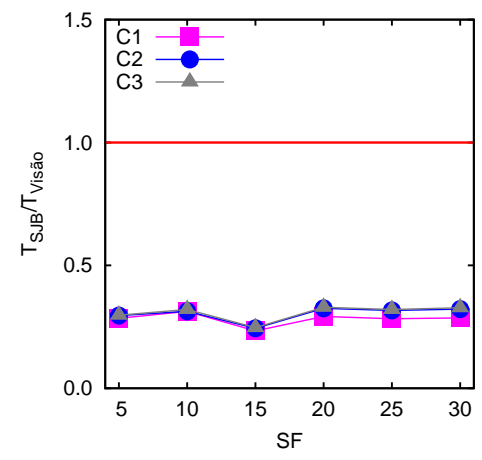

(k) Consulta Q6.3.

Figura 6.8: Comparação do desempenho entre visão materializada e a estratégia SJB para os conjuntos Q4, Q5 e Q6 variando o fator de escala (SF) convencional. 


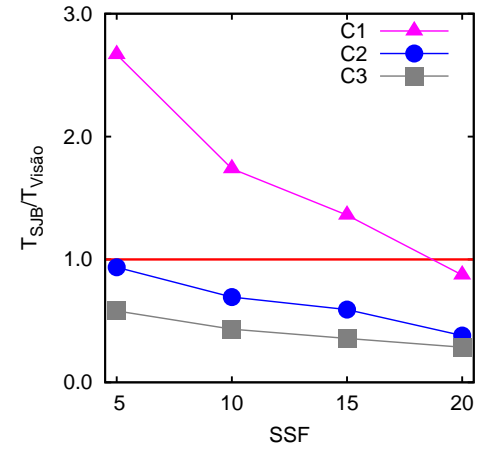

(a) Consulta Q1.1.

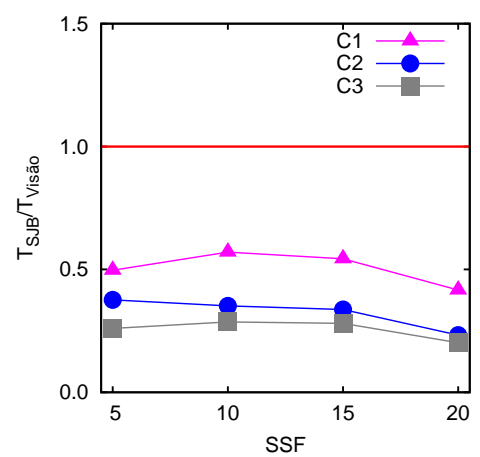

(b) Consulta Q1.2.

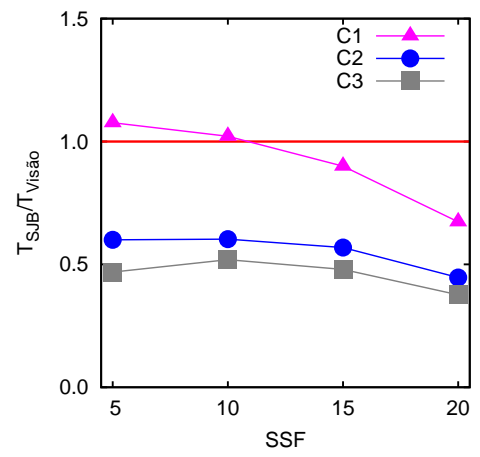

(c) Consulta Q1.3.

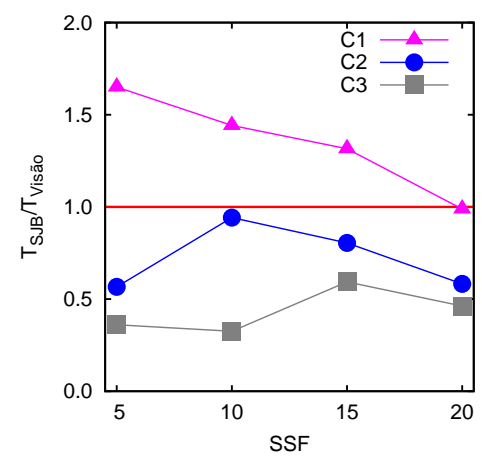

(d) Consulta Q1.4.

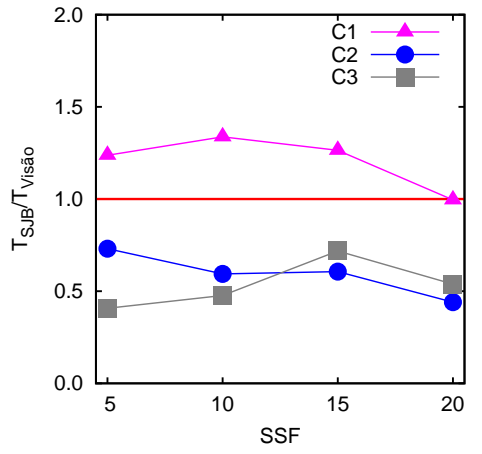

(e) Consulta Q2.1.

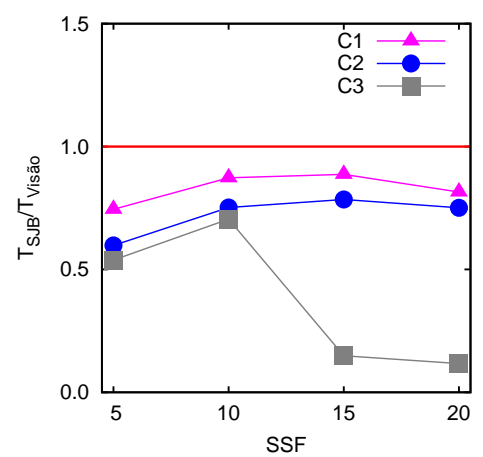

(f) Consulta Q2.2.

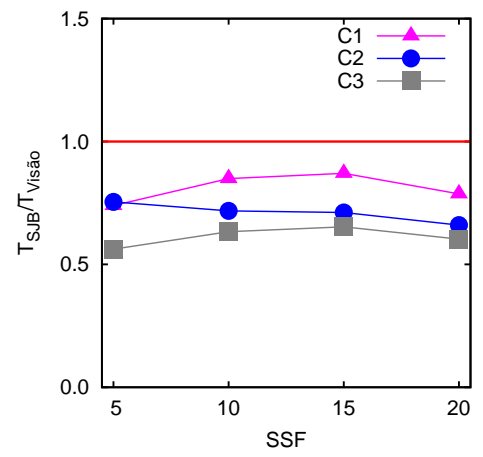

(g) Consulta Q2.3.

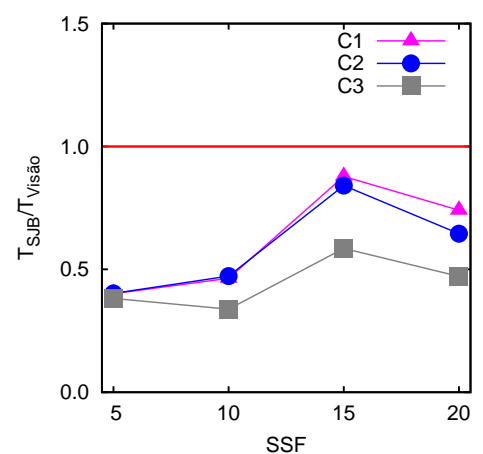

(h) Consulta Q2.4.

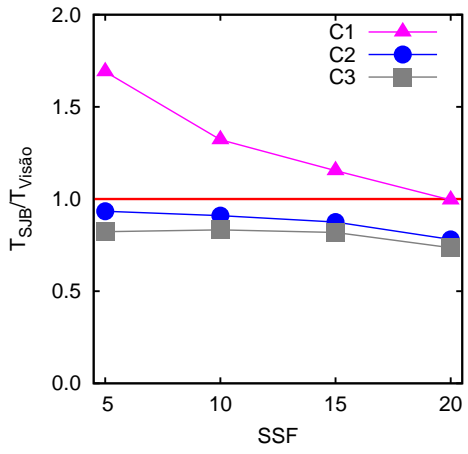

(i) Consulta Q3.1.

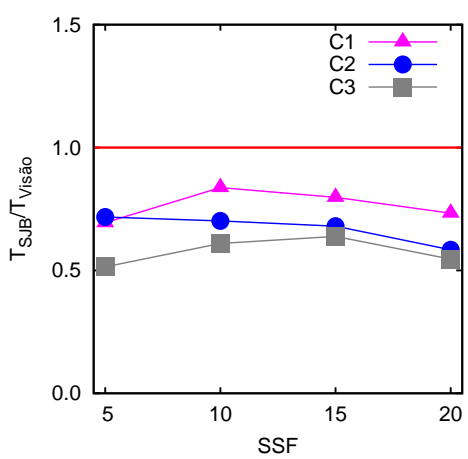

(j) Consulta Q3.2.

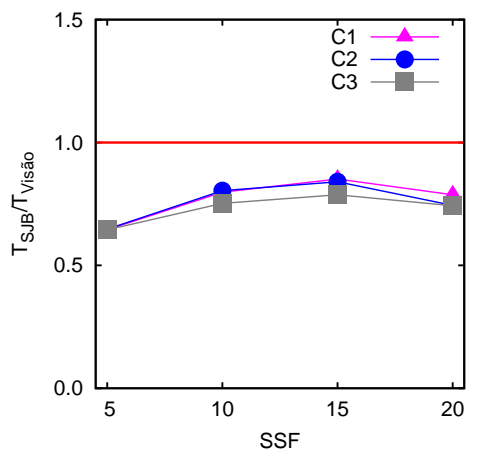

(k) Consulta Q3.3.

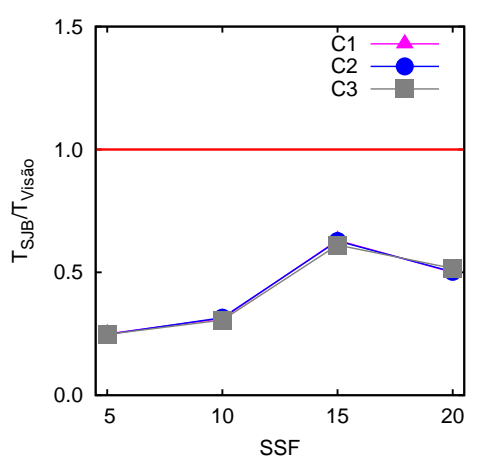

(I) Consulta Q3.4.

Figura 6.9: Comparação do desempenho entre visão materializada e a estratégia SJB para os conjuntos Q1, Q2 e Q3 variando o fator de escala espacial (SSF). 


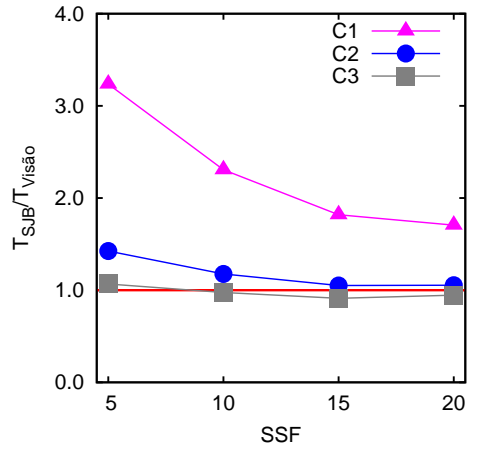

(a) Consulta Q4.1.

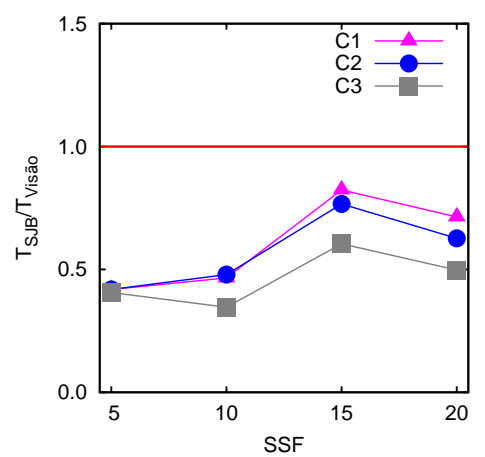

(b) Consulta Q4.2.

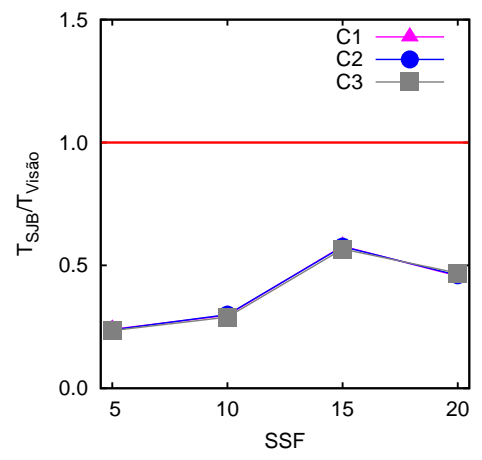

(c) Consulta Q4.3.

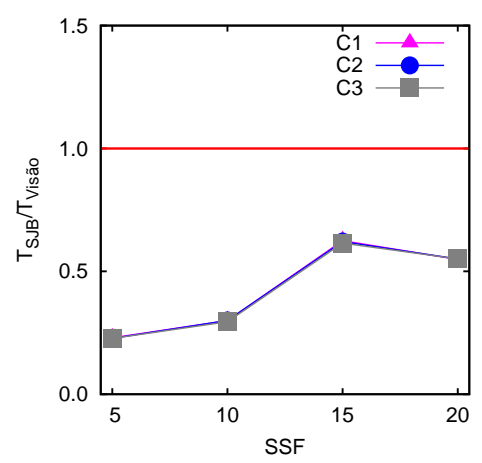

(d) Consulta Q4.4.

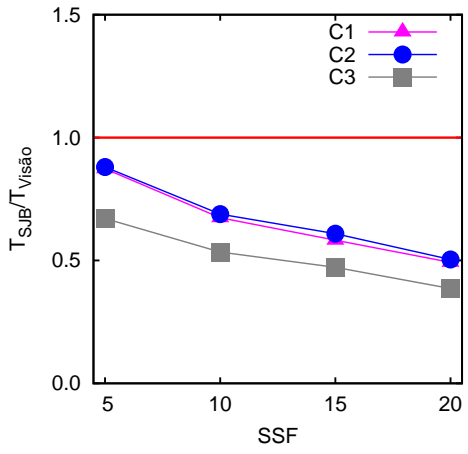

(e) Consulta Q5.1.

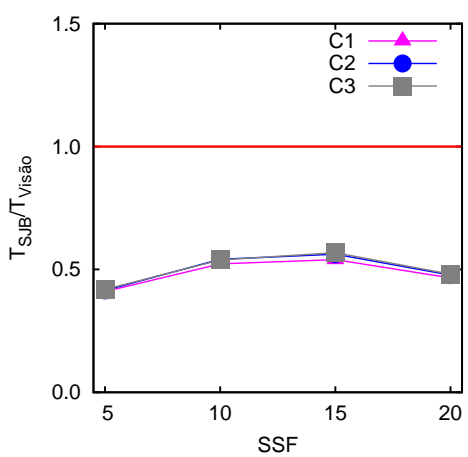

(f) Consulta Q5.2.

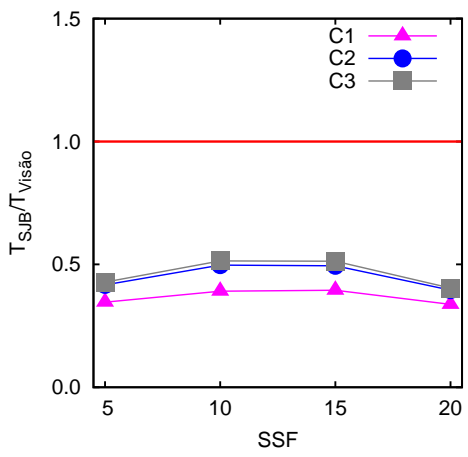

(g) Consulta Q5.3.

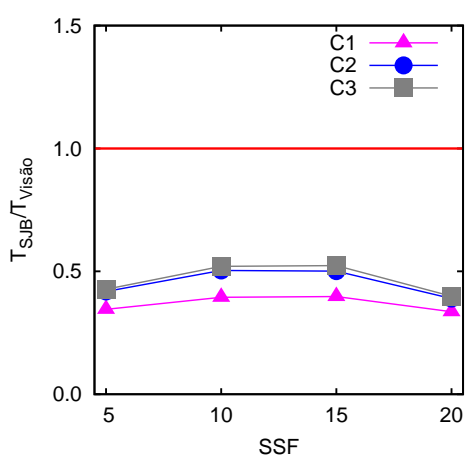

(h) Consulta Q5.4.

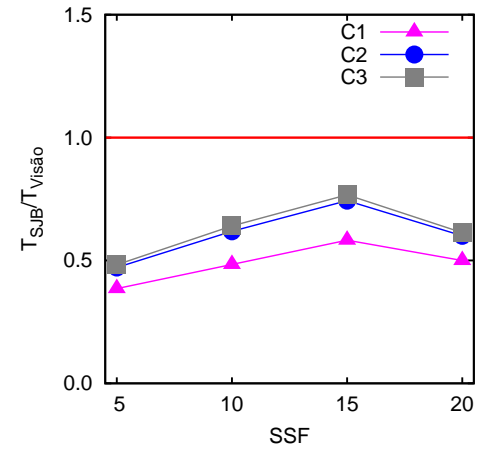

(i) Consulta Q6.1.

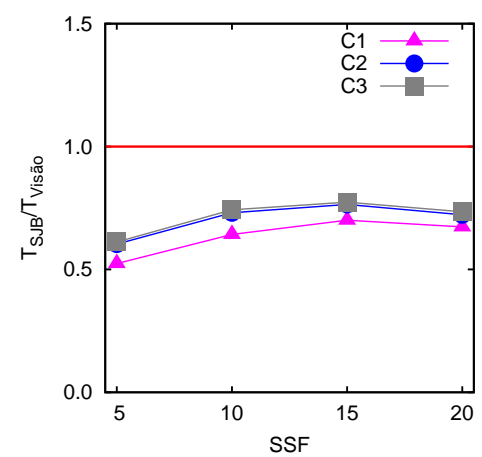

(j) Consulta Q6.2.

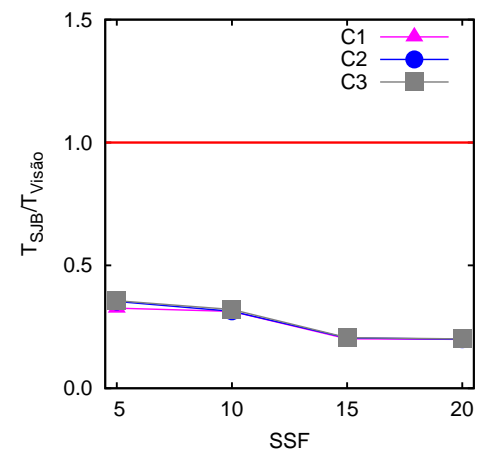

(k) Consulta Q6.3.

Figura 6.10: Comparação do desempenho entre visão materializada e a estratégia SJB para os conjuntos Q4, Q5 e Q6 variando o fator de escala espacial (SSF). 


\subsection{Considerações Finais}

Neste capítulo foi apresentada a estratégia SJB para o processamento de consultas SOLAP com junção espacial em DWGs, a qual é baseada no índice para DWG SB-index. A estratégia proposta baseia-se na redução do custo de processamento do predicado espacial, selecionando apenas pares de objetos espaciais de interesse.

Adicionalmente, visando oferecer fundamentação para a estratégia proposta, foram pesquisados dois aspectos relacionados, os quais referem-se: (i) à identificação de quais características o esquema de DWG deve possuir para possibilitar a execução de consultas SOLAP com junção espacial; e (ii) à especificação do formato de consultas SOLAP com junção espacial, de forma que essas consultas sejam executadas sobre um DWG projetado de acordo com as características identificadas.

A estratégia SJB foi contrastada contra a junção estrela e o uso de visões materializadas. Nos testes de desempenho realizados, a estratégia SJB garantiu um desempenho superior ao dos concorrentes na maioria das consultas. Para essas consultas, o ganho de desempenho variou de $2,5 \%$ até $99,2 \%$. Pode-se concluir que a estratégia proposta é a melhor opção a ser utilizada, principalmente se as consultas possuírem predicados convencionais com seletividade baixa.

No próximo capítulo é apresentada a conclusão desta dissertação de mestrado. 


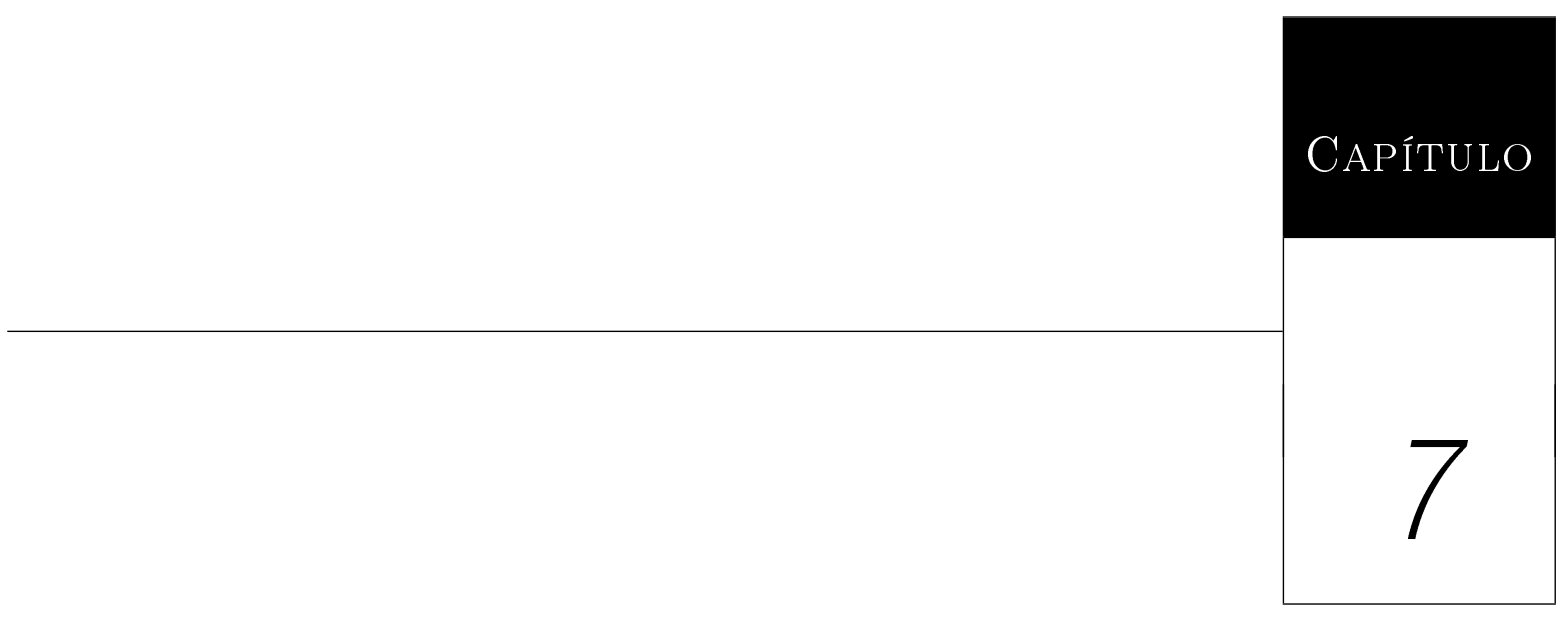

\section{Conclusões}

Esta dissertação teve como objetivo desenvolver estratégias voltadas ao processamento eficiente de consultas SOLAP drill-across e consultas SOLAP com junção espacial em data warehouses geográficos.

As estratégias propostas são baseadas no SB-index, o qual, segundo o nosso conhecimento, é o índice espacial para data warehouses geográficos mais eficiente existente na literatura até o momento. As estratégias de processamento que foram desenvolvidas consideram as seguintes características do SB-index: (i) enfoque em hierarquia espacial predefinida; (ii) reuso das características e funcionalidades do índice Bitmap de junção; e (iii) computação do predicado espacial primeiro, posterior transformação da resposta do predicado espacial em um predicado convencional, e processamento do predicado convencional gerado usando-se um índice Bitmap de junção.

Com relação ao processamento de consultas SOLAP drill-across, foram desenvolvidas duas estratégias: Divide e Única. A estratégia Divide baseia-se na divisão da constelação de fatos em vários esquemas estrela, de forma que cada esquema estrela seja indexado separadamente, e na divisão de uma consulta em várias subconsultas gerando vários subresultados, os quais são subsequentemente combinados, agrupados e ordenados para se obter o resultado final. A estratégia Única baseia-se na criação de um único índice para a constelação de fatos completa, e na consequente geração de um único subresultado, o qual é agrupado e ordenado para obter o resultado final da consulta. Adicionalmente, visando oferecer fundamentação para as estratégias propostas, foi feita a especificação de um conjunto de diretrizes que deve ser 
seguido para o projeto de um esquema de data warehouse geográfico que possibilite a execução de consultas SOLAP drill-across, além da especificação de classes de consultas SOLAP drillacross para serem executadas sobre esse esquema, as quais enfocam na análise de diferentes características das consultas, impondo dessa forma diferentes custos de processamento.

Para o processamento de consultas SOLAP com junção espacial foi proposta a estratégia $\mathrm{SJB}$, a qual é caracterizada por utilizar os predicados convencionais da consulta para reduzir o custo de processamento do predicado espacial. Visando oferecer fundamentação para a estratégia proposta, também foram identificadas quais características o esquema de data warehouse geográfico deve possuir para possibilitar a execução de consultas SOLAP com junção espacial e foi especificado o formato de consultas SOLAP com junção espacial, de forma que essas consultas sejam executadas sobre um data warehouse geográfico projetado de acordo com as características identificadas.

A validação das estratégias de processamento de consultas SOLAP drill-across e consultas SOLAP com junção espacial propostas nesta dissertação foi realizada por meio de testes de desempenho considerando diferentes volumes de dados armazenados no data warehouse geográfico e diferentes seletividades de consultas. Os resultados obtidos foram contrastados com a execução de consultas do tipo junção estrela e o uso de visões materializadas.

Os testes de desempenho com as estratégias Divide e Única mostraram que ambas são muito mais eficientes que o uso da junção estrela e de visões materializadas no processamento de consultas SOLAP drill-across. Os resultados mostraram que as abordagens Divide e Única melhoraram o desempenho no processamento de consultas SOLAP drill-across de $82,7 \%$ a $98,6 \%$. Nos testes de desempenho realizados, verificou-se que a estratégia Única deve ser usada quando a proporção entre as tabelas de fatos é de 1:1. Nessa proporção, essa estratégia apresentou uma redução no tempo de execução com relação à estratégia Divide que variou de $8,2 \%$ a $49,2 \%$. Para as proporções $1: 3,1: 4$ e 1:5, a estratégia Divide apresentou um desempenho superior, reduzindo o tempo de execução das consultas de 5,6\% a 42,6\% com relação à estratégia Única.

Os testes de desempenho com a estratégia SJB mostraram que ela é uma excelente opção para o processamento de consultas SOLAP com junção espacial que possuam predicados convencionais com seletividade baixa. A estratégia SJB mostrou ser mais eficiente que o uso da junção estrela e da visão materializada, mesmo quando essas dispõem de índices (Btrees) nos atributos envolvidos nos predicados convencionais. Os resultados dos testes de desempenho mostraram que, para a grande maioria das consultas, a estratégia SJB garantiu uma melhora de desempenho com relação à junção estrela e ao uso de visões materializadas. Para uma das configurações utilizadas, na qual os predicados convencionais recuperam $0,15 \%$ das tuplas, a estratégia SJB reduziu o tempo de execução de $1,0 \%$ a 99,2\% em relação à junção estrela e ao uso de visões materializadas. 


\subsection{Contribuições}

As principais contribuições desta dissertação de mestrado são:

- Proposta de diretrizes para projetar esquemas de data warehouses geográficos que possibilitem a execução de consultas SOLAP drill-across.

- Proposta de classes de consultas SOLAP drill-across.

- Proposta das estratégias Divide e Única, duas estratégias eficientes para o processamento de consultas SOLAP drill-across.

- Identificação de cenários nos quais as estratégias Divide e Única são mais apropriadas para serem utilizadas.

- Proposta da estratégia SJB, uma estratégia eficiente para o processamento de consultas SOLAP com junção espacial.

- Proposta de características que esquemas de data warehouses geográficos devem possuir para possibilitar a execução de consultas SOLAP com junção espacial.

- Proposta do formato de consultas SOLAP com junção espacial.

\subsection{Trabalhos Futuros}

Trabalhos futuros relativos à extensão das estratégias de processamento propostas incluem:

- Identificação de outros cenários nos quais a estratégia Única seja mais eficiente que a estratégia Divide.

- Investigação do desempenho das três estratégias mediante a substituição do SB-index pelo HSB-index.

- Investigação do desempenho das estratégias Divide, Única e SJB em diferentes esquemas, consultas, tipos de dados espaciais e predicados espaciais.

- Investigação do desempenho da estratégia SJB considerando um número maior de configurações dos predicados convencionais.

- Utilização da estratégia SJB em conjunto com as estratégias Divide e Única para resolver consultas SOLAP drill-across com junção espacial. 


\section{Referências Bibliográficas}

Agrawal, S.; Chaudhuri, S.; NArasayya, V. R. Automated selection of materialized views and indexes in SQL databases. In: INTERNATIONAL CONFERENCE ON VERY LARGE DATA BASES, 26., 2000, Cairo, Egypt. Proceedings... San Francisco, CA, USA: Morgan Kaufmann Publishers Inc., 2000. p. 496-505.

Antoshenkov, G. Byte-aligned bitmap compression. In: CONFERENCE ON DATA COMPRESSION, 1995, Snowbird, UT, USA. Proceedings... Washington, DC, USA: IEEE Computer Society, 1995. p. 476.

AREF, W. G. Pipelined spatial join processing for quadtree-based indexes. In: ACM INTERNATIONAL SYMPOSIUM ON GEOGRAPHIC INFORMATION SYSTEMS, 15., 2007, Seattle, Washington. Proceedings... New York, NY, USA: ACM, 2007. p. 49.

Back, H.; Won, J.-I.; YoOn, J.; PARK, S.; KIM, S.-W. Efficient processing of spatial joins with DOT-based indexing. Information Sciences, v. 180, n. 8, p. 1292-1312, 2010.

Bae, W. D.; Alkobaisi, S.; Leutenegger, S. T. An incremental refining spatial join algorithm for estimating query results in GIS. In: INTERNATIONAL CONFERENCE ON DATABASE AND EXPERT SYSTEMS APPLICATIONS, 17., 2006, Kraków, Poland. Proceedings... Berlin/Heidelberg, Germany: Springer-Verlag, 2006. p. 935-944.

Bae, W. D.; Alkobaisi, S.; Leutenegger, S. T. IRSJ: incremental refining spatial joins for interactive queries in gis. Geolnformatica, v. 14, n. 4, p. 507-543, 2010.

BAIKOUsi, E.; VAssiliadis, P. View usability and safety for the answering of top-k queries via materialized views. In: INTERNATIONAL WORKSHOP ON DATA WAREHOUSING AND OLAP, 12., 2009, Hong Kong, China. Proceedings... New York, NY, USA: ACM, 2009. p. $97-104$

Baralis, E.; Paraboschi, S.; Teniente, E. Materialized views selection in a multidimensional database. In: INTERNATIONAL CONFERENCE ON VERY LARGE DATA BASES, 23., 1997, Athens, Greece. Proceedings... San Francisco, CA, USA: Morgan Kaufmann Publishers Inc., 1997. p. 156-165. 
Beckmann, N.; Kriegel, H.-P.; Schneider, R.; Seeger, B. The R*-tree: An efficient and robust access method for points and rectangles. In: ACM SIGMOD INTERNATIONAL CONFERENCE ON MANAGEMENT OF DATA, 1990, Atlantic City, NJ. Proceedings... New York, NY, USA: ACM, 1990. p. 322-331.

Bellatreche, L.; Woameno, K. Y. Dimension table driven approach to referential partition relational data warehouses. In: INTERNATIONAL WORKSHOP ON DATA WAREHOUSING AND OLAP, 12., 2009, Hong Kong, China. Proceedings... New York, NY, USA: ACM, 2009. p. 9-16.

Benkrid, S.; Bellatreche, L.; Drias, H. A combined selection of fragmentation and allocation schemes in parallel data warehouses. In: INTERNATIONAL CONFERENCE ON DATABASE AND EXPERT SYSTEMS APPLICATIONS, 19., 2008, Turin, Italy. Proceedings... Washington, DC, USA: IEEE Computer Society, 2008. p. 370-374.

Bimonte, S.; TChounikine, A.; Miquel, M. Towards a spatial multidimensional model. In: INTERNATIONAL WORKSHOP ON DATA WAREHOUSING AND OLAP, 8., 2005, Bremen, Germany. Proceedings... New York, NY, USA: ACM, $2005 . \quad$ p. 39-46.

Brinkhoff, T.; Kriegel, H.-P.; Schneider, R.; SeEger, B. Multi-step processing of spatial joins. In: ACM SIGMOD INTERNATIONAL CONFERENCE ON MANAGEMENT OF DATA, 1994, Minneapolis, Minnesota, United States. Proceedings... New York, NY, USA: ACM, 1994. p. 197-208.

Brinkhoff, T.; Kriegel, H.-P.; SEeger, B. Efficient processing of spatial joins using R-trees. In: ACM SIGMOD INTERNATIONAL CONFERENCE ON MANAGEMENT OF DATA, 1993, Washington, D.C.. Proceedings... New York, NY, USA: ACM, $1993 . \quad$ p. 237-246.

Brito, J. J.; Siqueira, T. L. L.; Times, V. C.; Ciferri, R. R.; Ciferri, C. D. A. Efficient processing of drill-across queries over geographic data warehouses. In: DaWaK, 2011. p. $152-166$.

Chan, C. Y.; IOANNIDIS, Y. E. An efficient bitmap encoding scheme for selection queries. In: ACM SIGMOD INTERNATIONAL CONFERENCE ON MANAGEMENT OF DATA, 1999, Philadelphia, Pennsylvania. Proceedings... New York, NY, USA: ACM, 1999. p. $215-226$.

Chaudhuri, S.; Dayal, U. An overview of data warehousing and olap technology. ACM SIGMOD Record, v. 26, n. 1, p. 65-74, 1997.

Chen, H.-L.; Chang, Y.-I. Spatial joins based on na-trees. Information Processing Letters, v. 109, n. 13, p. 713-718, 2009. 
Ciferri, C. D. A.; Ciferri, R. R.; Forlani, D. T.; Traina, A. J. M.; Souza, F. F. Horizontal fragmentation as a technique to improve the performance of drill-down and roll-up queries. In: ACM SYMPOSIUM ON APPLIED COMPUTING, 2007, Seoul, Korea. Proceedings... New York, NY, USA: ACM, 2007. p. 494-499.

Ciferri, C. D. A.; SouzA, F. F. Data warehousing: Estado da arte e direções futuras. In: CONFERÊNCIA LATINO-AMERICANA DE INFORMÁTICA, 26., 2000, Mexico City, Mexico. Memórias... p. p.12.

CIfERri, R. R. Análise da influência do fator distribuição espacial dos dados no desempenho de métodos de acesso multidimensionais. Tese (Doutorado em Ciências da Computação), UFPE, Recife, Brasil, 2002.

Costa, M.; MAdeIRA, H. Handling big dimensions in distributed data warehouses using the DWS technique. In: INTERNATIONAL WORKSHOP ON DATA WAREHOUSING AND OLAP, 7., 2004, Washington, DC, USA. Proceedings... New York, NY, USA: ACM, 2004. p. 31-37.

Derakhshan, R.; Stantic, B.; Korn, O.; Dehne, F. K. H. A. Parallel simulated annealing for materialized view selection in data warehousing environments. In: INTERNATIONAL CONFERENCE ON ALGORITHMS AND ARCHITECTURES FOR PARALLEL PROCESSING, 8., 2008, Cyprus. Proceedings... Berlin/Heidelberg, Germany: SpringerVerlag, 2008. p. 121-132.

Fidalgo, R. N.; Times, V. C.; Silva, J.; Souza, F. F. GeoDWFrame: A framework for guiding the design of geographical dimensional schemas. In: INTERNATIONAL CONFERENCE ON DATA WAREHOUSING AND KNOWLEDGE DISCOVERY, 6., 2004, Zaragoza, Spain. Proceedings... Berlin/Heidelberg, Germany: Springer-Verlag, 2004. p. 26-37.

Gaede, V.; Günther, O. Multidimensional access methods. ACM Computing Surveys, v. 30, n. 2, p. $170-231,1998$.

Gatti, S. D.; Magalhães, G. C. An investigation on factors that affect the performance of spatial joins. In: SIMPÓSIO BRASILEIRO DE BANCO DE DADOS, 15., 2000, João Pessoa, Paraíba, Brasil. Anais... João Pessoa, Paraíba: CEFET-PB, $2000 . \quad$ p. 130-142.

Golfarelli, M.; Maniezzo, V.; Rizzi, S. Materialization of fragmented views in multidimensional databases. Data \& Knowledge Engineering, v. 49, n. 3, p. 325-351, $2004 b$.

Golfarelli, M.; Rizzi, S. A survey on temporal data warehousing. International Journal of Data Warehousing and Mining, v. 5, n. 1, p. 1-17, 2009. 
Golfarelli, M.; Rizzi, S.; Cella, I. Beyond data warehousing: What's next in business intelligence? In: INTERNATIONAL WORKSHOP ON DATA WAREHOUSING AND OLAP, 7., 2004a, Washington, DC, USA. Proceedings... New York, NY, USA: ACM, 2004a. p. 1-6.

Goyal, N.; ZAVERI, S. K.; ShARMA, Y. Improved bitmap indexing strategy for data warehouses. In: INTERNATIONAL CONFERENCE ON INFORMATION TECHNOLOGY, 9., 2006, Bhubaneswar, Orissa, India. Proceedings... Washington, DC, USA: IEEE Computer Society, 2006. p. 213-216.

GÜNTHER, O. Efficient computation of spatial joins. In: INTERNATIONAL CONFERENCE ON DATA ENGINEERING, 9., 1993, Vienna, Austria. Proceedings... Washington, DC, USA: IEEE Computer Society, 1993. p. 50-59.

Günther, O.; Picouet, P.; SAglio, J.-M.; Scholl, M.; Oria, V. Benchmarking spatial joins a la carte. International Journal of Geographical Information Science, v. 13, n. 7, p. $639-655,1999$.

Gupta, H.; Mumick, I. S. Selection of views to materialize under a maintenance cost constraint. In: INTERNATIONAL CONFERENCE ON DATABASE THEORY, 7., 1999, Jerusalem, Israel. Proceedings... London, UK: Springer-Verlag, 1999. p. 453-470.

Guttman, A. R-trees: A dynamic index structure for spatial searching. ACM SIGMOD Record, v. 14, n. 2, p. 47-57, 1984.

Harinarayan, V.; Rajaraman, A.; Ullman, J. D. Implementing data cubes efficiently. ACM SIGMOD Record, v. 25, p. 205-216, 1996.

Hung, M.-C.; HuAng, M.-L.; YAng, D.-L.; HsueH, N.-L. Efficient approaches for materialized views selection in a data warehouse. Information Sciences, v. 177, n. 6 , p. 1333-1348, 2007.

Inmon, W. H. Building the Data Warehouse. 3 ed. Wiley Computer Publishing, 412 p., 2002.

JACOX, E. H.; SAMET, H. Iterative spatial join. ACM Transactions on Database Systems, v. 28, n. 3, p. 230-256, 2003.

JACOX, E. H.; SAMET, H. Spatial join techniques. ACM Transactions on Database Systems, v. 32, n. 1, p. 7, 2007.

Johnson, T.; Shasha, D. Some approaches to index design for cube forest. IEEE Data Engineering Bulletin, v. 20, n. 1, p. 27-35, 1997. 
Kimball, R.; Ross, M. The Data Warehouse Toolkit: The complete guide to dimensional modeling. 2 ed. Wiley Computer Publishing, 2002.

Kotidis, Y.; Roussopoulos, N. A case for dynamic view management. ACM Transactions on Database Systems, v. 26, n. 4, p. 388-423, 2001.

LaWrence, M.; Rau-Chaplin, A. Dynamic view selection for olap. In: INTERNATIONAL CONFERENCE ON DATA WAREHOUSING AND KNOWLEDGE DISCOVERY, 8., 2006, Krakow, Poland. Proceedings... Berlin/Heidelberg, Germany: Springer-Verlag, 2006. p. 33-44.

Malinowski, E.; ZimánYI, E. Representing spatiality in a conceptual multidimensional model. In: ACM INTERNATIONAL WORKSHOP ON GEOGRAPHIC INFORMATION SYSTEMS, 12., 2004, Washington DC, USA. Proceedings... New York, NY, USA: ACM, 2004. p. $12-22$.

Malinowski, E.; ZimÁNYI, E. Spatial hierarchies and topological relationships in the spatial MultiDimER model. In: BRITISH NATIONAL CONFERENCE ON DATABASES, 22., 2005, Sunderland, UK. Proceedings... Berlin/Heidelberg, Germany: Springer-Verlag, 2005. p. $17-28$.

Mamoulis, N. Spatial join. In: Encyclopedia of Database Systems, 2009, p. 27072714.

Mamoulis, N.; Theodoridis, Y.; Papadias, D. Spatial joins: Algorithms, cost models and optimization techniques. In: Manolopoulos, Y.; PAPAdopoulos, A.; VAssilakopoulos, M., eds. Spatial Databases: Technologies, Techniques and Trends, Harpenden, Hertfordshire, UK: Idea Group, 2005, cap. 7, p. 155-184.

Mateus, R. C.; Siqueira, T. L. L.; Times, V. C.; Ciferri, R. R.; Ciferri, C. D. A. How does the spatial data redundancy affect query performance in geographic data warehouses? Journal of Information and Data Management, v. 1, n. 3, p. 519-534, 2010 .

Mohan, P.; Wilson, R. E.; Shekhar, S.; George, B.; Levine, N.; Celik, M. Should sdbms support a join index?: a case study from crimestat. In: ACM SIGSPATIAL INTERNATIONAL SYMPOSIUM ON ADVANCES IN GEOGRAPHIC INFORMATION SYSTEMS, 16., 2008, Irvine, California, USA. Proceedings... New York, NY, USA: ACM, 2008. p. 37.

O'NEIL, P. E. Model 204 architecture and performance. In: INTERNATIONAL WORKSHOP ON HIGH PERFORMANCE TRANSACTION SYSTEMS, 2., 1987, Pacific Grove, California. Proceedings... Berlin/Heidelberg, Germany: Springer-Verlag, 1987. p. $40-59$. 
O’Neil, P. E.; Graefe, G. Multi-table joins through bitmapped join indices. ACM SIGMOD Record, v. 24, n. 3, p. 8-11, 1995.

O'NeIL, P. E.; QuAss, D. Improved query performance with variant indexes. In: ACM INTERNATIONAL CONFERENCE ON MANAGEMENT OF DATA, 1997, Tucson, USA. Proceedings... New York, NY, USA: ACM, 1997. p. 38-49.

Papadias, D.; Kalnis, P.; Zhang, J.; TAO, Y. Efficient olap operations in spatial data warehouses. In: INTERNATIONAL SYMPOSIUM ON SPATIAL AND TEMPORAL DATABASES, 7., 2001, Redondo Beach, USA. Proceedings... London, UK: SpringerVerlag, 2001. p. 443-459.

PAPAdias, D.; TAO, Y.; Kalnis, P.; Zhang, J. Indexing spatio-temporal data warehouses. In: INTERNATIONAL CONFERENCE ON DATA ENGINEERING, 18., 2002, Washington, DC. Proceedings... Washington, DC, USA: IEEE Computer Society, 2002. p. $166-175$.

Papadopoulos, A.; Rigaux, P.; Scholl, M. A performance evaluation of spatial join processing strategies. In: INTERNATIONAL SYMPOSIUM ON ADVANCES ON SPATIAL DATABASES, 6., 1999, Hong Kong, China. Proceedings... Berlin/Heidelberg, Germany: Springer-Verlag, 1999. p. 286-307.

PÖsS, M.; FlOYD, C. New tpc benchmarks for decision support and web commerce. SIGMOD Record, v. 29, n. 4, p. 64-71, 2000.

RochA, S. R. Análise de desempenho de junções espaciais: uma comparação entre os métodos analítico e experimental. Dissertação (Mestrado em Ciências da Computação), UNICAMP, Campinas, Brasil, 2003.

Rotem, D.; Stockinger, K.; Wu, K. Optimizing candidate check costs for bitmap indices. In: INTERNATIONAL CONFERENCE ON INFORMATION AND KNOWLEDGE MANAGEMENT, 2005, Bremen, Germany. Proceedings... New York, NY, USA: ACM, 2005. p. 648-655.

Roussopoulos, N.; LeIfKer, D. Direct spatial search on pictorial databases using packed R-trees. In: INTERNATIONAL CONFERENCE ON MANAGEMENT OF DATA, 1985, Austin, Texas. Proceedings... New York, NY, USA: ACM, $1985 . \quad$ p. 17-31.

Sampaio, M. C.; Sousa, A. G.; BAptista, C. S. Towards a logical multidimensional model for spatial data warehousing and OLAP. In: INTERNATIONAL WORKSHOP ON DATA WAREHOUSING AND OLAP, 9., 2006, Arlington, USA. Proceedings... New York, NY, USA: ACM, 2006. p. 83-90. 
Samtani, S.; Mohania, M.; Kumar, V.; Kambayashi, Y. Recent advances and research problems in data warehousing. Lecture Notes in Computer Science, v. 1552, p. 83-90, 1998.

Sarawagi, S. Indexing olap data. Data Engineering Bulletin, v. 20, n. 1, p. 36-43, 1997.

Shah, B.; Ramachandran, V.; Ramachandran, K. A hybrid approach for data warehouse view selection. International Journal of Data Warehousing and Mining, v. 2, n. 2, p. 1-37, 2006.

Silva, J.; Times, V. C.; Salgado, A. C. An open source and web based framework for geographic and multidimensional processing. In: ACM SYMPOSIUM ON APPLIED COMPUTING, 21., 2006, Dijon, France. Proceedings... New York, NY, USA: ACM, 2006. p. 63-67.

Silva, J.; Times, V. C.; Salgado, A. C.; Souza, C.; Fidalgo, R. N.; Oliveira, A. G. A set of aggregation functions for spatial measures. In: INTERNATIONAL WORKSHOP ON DATA WAREHOUSING AND OLAP, 11., 2008, Napa Valley, California, USA. Proceedings... New York, NY, USA: ACM, 2008. p. 25-32.

SiqueirA, T. L. L. SB-Index: um índice espacial baseado em Bitmap para data warehouse geográfico. Dissertação (Mestrado em Ciências da Computação), UFSCAR, São Carlos, Brasil, 2009.

Siqueira, T. L. L.; De Aguiar Ciferri, C. D.; Times, V. C.; Ciferri, R. R. The SB-index and the HSB-index: efficient indices for spatial data warehouses. Geolnformatica, v. 16, n. 1, p. 165-205, 2012.

Siqueira, T. L. L.; Ciferri, C. D. A.; Times, V. C.; Oliveira, A. G.; Ciferri, R. R. The impact of spatial data redundancy on solap query performance. Journal of the Brazilian Computer Society, v. 15, p. 19-34, $2009 \mathrm{~b}$.

Siqueira, T. L. L.; Ciferri, R. R.; Times, V. C.; Ciferri, C. D. A. Investigating the effects of spatial data redundancy in query performance over geographical data warehouses. In: BRAZILIAN SYMPOSIUM ON GEOINFORMATICS, 10., 2008, Rio de Janeiro, Brasil. Proceedings... p. 1-12.

Siqueira, T. L. L.; Ciferri, R. R.; Times, V. C.; Ciferri, C. D. A. A spatial bitmap-based index for geographical data warehouses. In: ACM SYMPOSIUM ON APPLIED COMPUTING, 24., 2009a, Honolulu, Hawaii, USA. Proceedings... New York, NY, USA: ACM, 2009a. p. 1336-1342. 
Siqueira, T. L. L.; Ciferri, R. R.; Times, V. C.; Ciferri, C. D. A. Benchmarking spatial data warehouses. In: INTERNATIONAL CONFERENCE ON DATA WAREHOUSING AND KNOWLEDGE DISCOVERY, 12., 2010, Bilbao, Spain. Proceedings... Berlin/Heidelberg: Springer-Verlag, 2010. p. 40-51.

Stefanovic, N.; Han, J.; Koperski, K. Object-based selective materialization for efficient implementation of spatial data cubes. IEEE Transactions on Knowledge and Data Engineering, v. 12, n. 6, p. 938-958, 2000.

Stockinger, K.; Wu, K.; Shoshani, A. Evaluation strategies for bitmap indices with binning. In: INTERNATIONAL CONFERENCE ON DATABASE AND EXPERT SYSTEMS APPLICATIONS, 15., 2004, Zaragoza, Spain. Proceedings... Berlin/Heidelberg: Spring, 2004. p. $120-129$.

VASSILIADIS, P. Gulliver in the land of data warehousing: practical experiences and observations of a researcher. In: INTERNATIONAL WORKSHOP ON DESIGN AND MANAGEMENT OF DATA WAREHOUSES, 2., 2000, Stockholm, Sweden. Proceedings... p. 12.

WIDOM, J. Research problems in data warehousing. In: INTERNATIONAL CONFERENCE ON INFORMATION AND KNOWLEDGE MANAGEMENT, 4., 1995, Baltimore, Maryland, USA. Proceeedings... New York, NY, USA: ACM, 1995. p. 25-30.

WU, K. FastBit: an efficient indexing technology for accelerating data-intensive science. Journal of Physics: Conference Series, v. 16, n. 1, p. 556-560, 2005.

Wu, K.; Otoo, E. J.; Shoshani, A. Optimizing bitmap indices with efficient compression. ACM Transactions on Database Systems, v. 31, n. 1, p. 1-38, 2006.

Wu, K.; Stockinger, K.; Shoshani, A. Breaking the curse of cardinality on bitmap indexes. In: INTERNATIONAL CONFERENCE ON SCIENTIFIC AND STATISTICAL DATABASE MANAGEMENT, 20., 2008, Hong Kong, China. Proceedings... Berlin/Heidelberg: Springer-Verlag, 2008. p. 348-365.

Wu, M.-C.; Buchmann, A. P. Research issues in data warehousing. In: THE GERMAN DATABASE CONFERENCE, 1997, Ulm, Germany. Proceedings... New York, NY, USA: ACM, 1997. p. 61-82.

Wu, M.-C.; BuCHMANN, A. P. Encoded bitmap indexing for data warehouses. In: INTERNATIONAL CONFERENCE ON DATA ENGINEERING, 14., 1998, Orlando, Florida, USA. Proceedings... Washington, DC, USA: IEEE Computer Society, 1998. p. 220-230. 